\title{
Modeling and Testing of a Mechanical Counterpressure BioSuit System
}

\author{
by \\ Daniel Clemente Louis Judnick \\ B.S.E. in Mechanical Engineering \\ Loyola Marymount University, 2005
}

\begin{abstract}
Submitted to the Department of Aeronautics and Astronautics in partial fulfillment of the requirements for the degree of

Master of Science in Aeronautics and Astronautics

at the

Massachusetts Institute of Technology
\end{abstract}

June 2007

(C) Massachusetts Institute of Technology 2007.

All rights reserved

Author

Department of Aeronautics and Astronautics

May 25, 2007

Certified by

Professor Jeffrey A. Hoffman

Professor of the Practice of Aerospace Engineering

Thesis Supervisor

Accepted by

Jaime Peraire

Professor of Aeronautics and Astronautics

Chair, Committee on Graduate Students 


\title{
Modeling and Testing of a Mechanical Counterpressure BioSuit System
}

\author{
by \\ Daniel Clemente Louis Judnick
}

submitted to the Department of Aeronautics and Astronautics on May 25, 2007, in partial fulfillment of the requirements for the degree of Master of Science in Aeronautics and Astronautics

\begin{abstract}
The President's Vision for Space Exploration calls for a returned human presence on the Moon, followed by human missions to Mars. The astronauts on these missions will require a more robust and flexible spacesuit than currently exists to conduct exploration and science operations as well as maintain a base on planetary surfaces.

The BioSuit system is a modular spacesuit concept based on the theory of mechanical counterpressure (MCP). Considerable experimental work has been conducted in the field of MCP, but there has been no analysis of the hypothetical best level of uniformity of pressure production for this type of spacesuit design. Therefore, computer modeling has been undertaken to verify the feasibility of such a design, which is based on not only providing the required pressure on the skin, but also limiting the variation of pressure production around a cross-section of the body. Given the data sets available, which exclusively consist of legs under normal atmospheric pressure, not mechanical counterpressure, the modeling work indicates that a MCP-based design can meet these requirements.

This thesis advances the BioSuit design by laying out the system level requirements, and also by setting requirements for the fabric and closure mechanism in order to design a working prototype. As part of this design process, the team has further developed the elastic bindings concept, which previously was designed to produce pressure only on the calf. Now the team has extended the design to protect the entire leg in an underpressurized environment. Based on blood pressure, skin temperature, heart rate, and qualitative comfort ratings, the bands have proven successful at protecting the leg (with the exception of some minor edema on the knee) at the desired underpressure $(-225 \mathrm{~mm} \mathrm{Hg})$ over a full hour. A simple knee brace which filled the concavities of the knee was also tested, and proved successful in preventing edema in one trial.
\end{abstract}

While the design is not yet capable of operational testing due to limitations in mobility, it is a valuable stepping point towards developing a full BioSuit system.

This work was supported by STTR Contract No. NNJ06JD82C.

Thesis Supervisor: Prof. Jeffrey A. Hoffman

Title: Professor of the Practice of Aerospace Engineering 


\section{Acknowledgments}

It may take a village to raise a child, but a small army is required to help a graduate student actually graduate. I do not how to begin to adequately thank the following people, as well as the many more individuals who have made a difference in my life at MIT.

First and foremost, I would like to thank my advisor, Prof. Jeffrey A. Hoffman. I was extremely excited to join the Man Vehicle Laboratory to work with an astronaut who flew 5 missions, and my experience has far exceeded even my greatest expectations. Jeff has always managed to set aside time in his extremely hectic schedule for research questions and discussions, while maintaining a good balance between guiding my research and allowing me the room to explore my ideas independently. His guidance on a variety of matters, from design questions to the job search process to the details of thesis writing, has helped me develop my talents as an engineer, and for that help I am extremely grateful.

Prof. Dava Newman also has earned my sincere thanks for her guidance, as well as her efforts to make sure I was funded, which is no easy matter in graduate school. She additionally has allowed me the opportunity to work with all sorts of unexpected people, such as film crews and journalists, that I never dreamed would occur.

I have an immense amount of respect and admiration for Elizabeth "Biff" Deems, my bus and systems co-lead on the Mars Gravity Biosatellite Program. She took considerable extra work and stress upon herself to make my life easier, and I shall forever be in her debt. She is an outstanding engineer, and more importantly, an even better person, whose friendship I treasure.

My dear friend Erika Wagner also deserves special recognition for all the help and guidance she has provided to me these past 2 years. Every time I had a problem or question, it seemed like Erika had the answer, and when I was frustrated she always found a way to cheer me up. I consider her a great role model, and if I am half as good at my future jobs as she is at hers, I will be quite pleased with myself.

All of the graduate students in the MVL also require significant thanks for bringing a lively and enjoyable atmosphere to work every day, which helped make my staggeringly long to-do list much easier to handle.

Much of this thesis would have never been completed without the help of my trusted UROPs: Xavier Gonzalez, Stephanie Couch, Brad Holschuh, and Matt Peterson. Stephanie in particular deserves attention for her willingness to perform the mind-numbing TekScan tests outlined in Chapter 4. In addition, my friends Xaver Paulig and Nicholas "Pepperdine" Granzella also assisted me on a number of occasions, and their willingness to help is much appreciated.

No list of acknowledgements would be complete without thanking my subjects for their infinite patience through the many hardware delays I encountered and for volunteering to help me graduate. This thesis would never have been possible without you! 
I am also very appreciative of the help of my predecessor, Liang Sim, who answered all my questions about the details of his work, and helped me think through some of the new problems I encountered. His assistance with my data analysis was invaluable as well.

Dave Graziosi of ILC Dover graciously provided me with drawings for a leg seal and the urethane coated nylon fabric used as a pressure bladder for my experiments.

I also need to express my eternal gratitude to Apple Inc. for inventing iTunes, without which I may have been hard-pressed to complete this thesis.

Last, but certainly not least, I would like to thank my family for their support of me in pursuit of my dreams, and accepting that I would be an absent brother/son for 2 years while I immersed myself in everything that MIT had to offer. I love you all, and I could not have done it without you. 


\section{Table of Contents}

1 Chapter 1: Introduction...................................................................................................... 14

2 Chapter 2: Background .......................................................................................................... 16

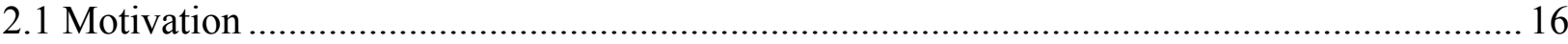

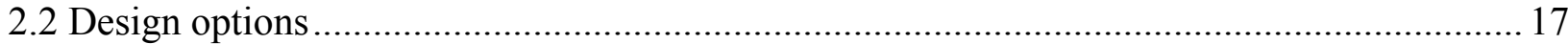

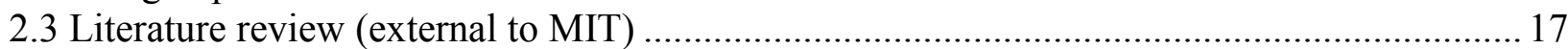

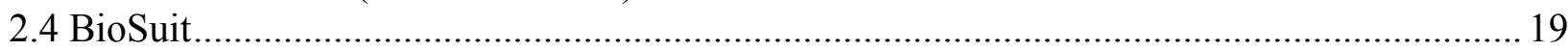

2.5 Further research into localized changes in pressure ............................................................22

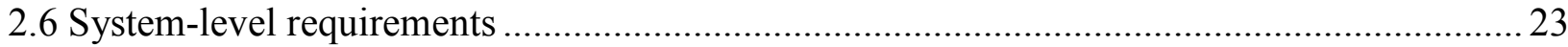

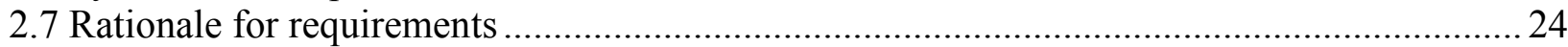

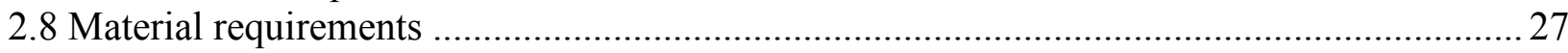

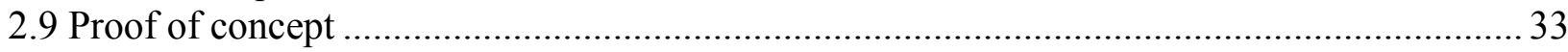

3 Chapter 3: Theoretical modeling of MCP in Matlab..............................................................37

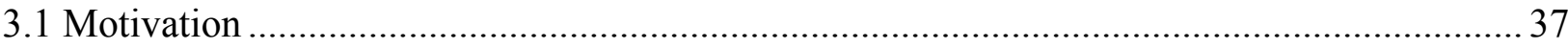

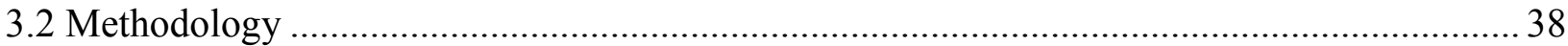

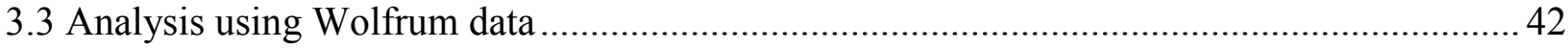

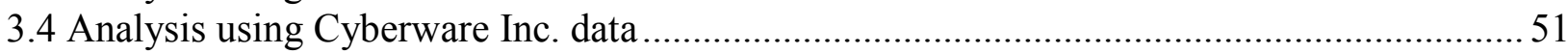

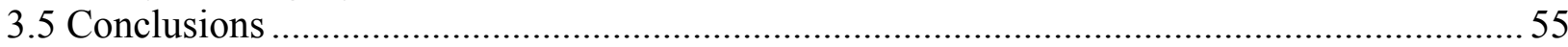

4 Chapter 4: TekScan testing ............................................................................................................5

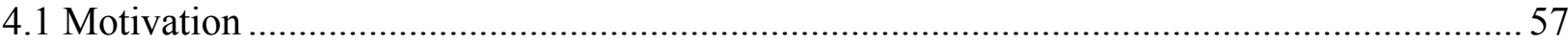

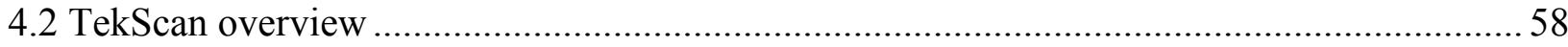

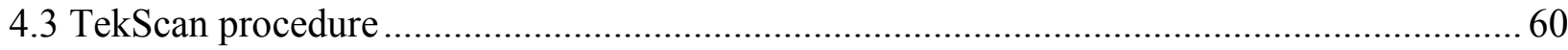

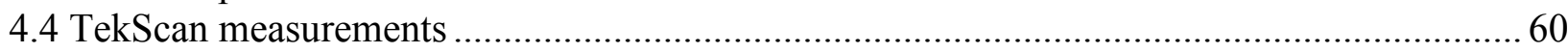

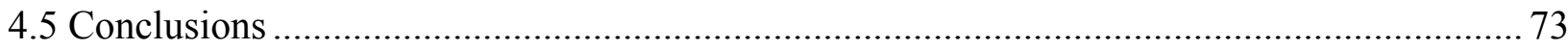

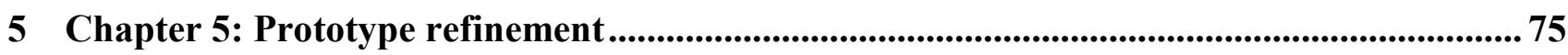

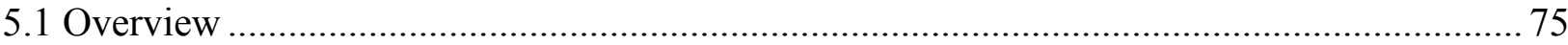

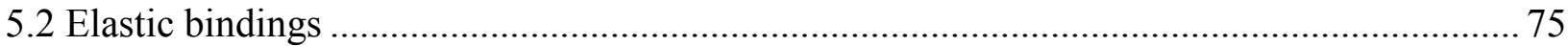

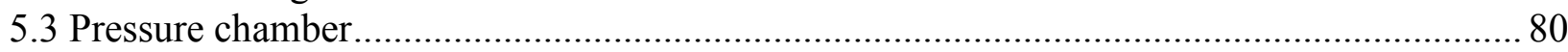

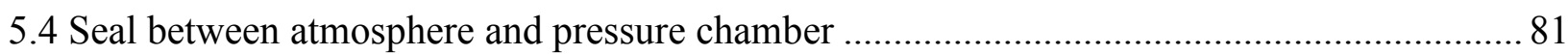

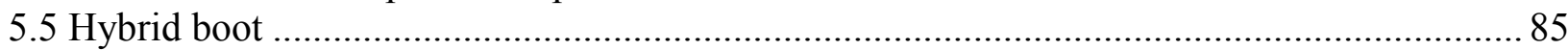

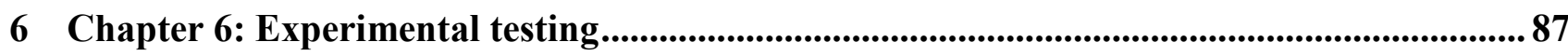

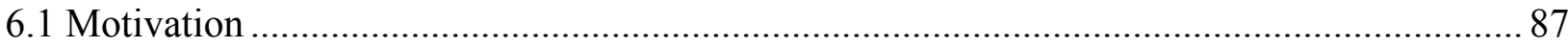

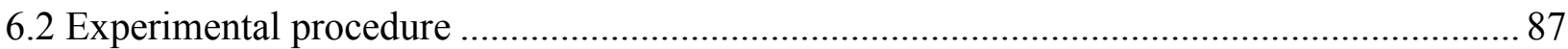

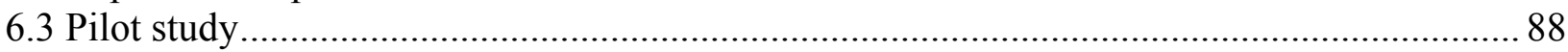

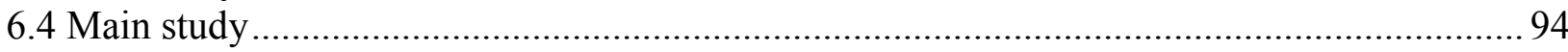

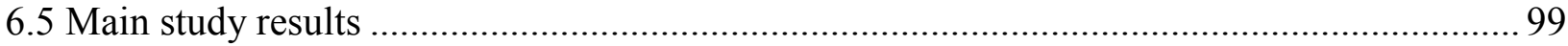

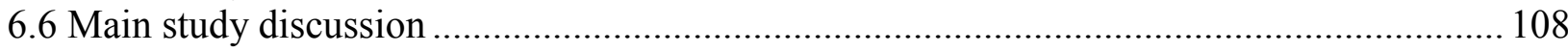

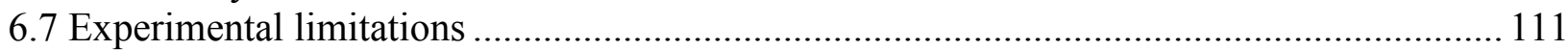

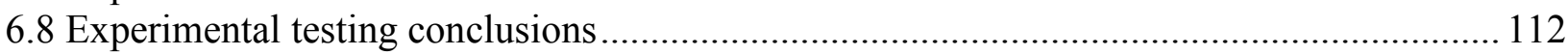

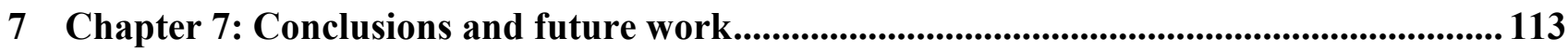

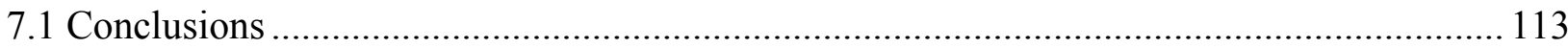

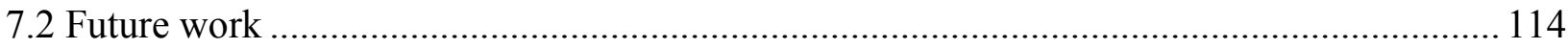

8 Appendix A: Derivation of Young's Modulus equations............................................................. 115 
9 Appendix B: Producing pressure over the knee with longitudinal bands ............................ 118

10 Appendix C: Full display of Young's Modulus ranges .................................................... 120

11 Appendix D: Matlab code for Young's Modulus theoretical calculations ........................... 122

12 Appendix E: Matlab code for analysis using Wolfrum data ............................................... 124

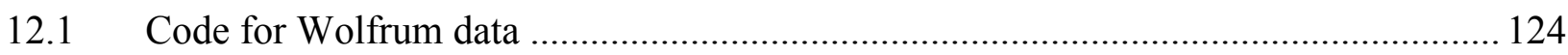

13 Appendix F: Matlab code for analysis using Cyberware data.......................................... 140

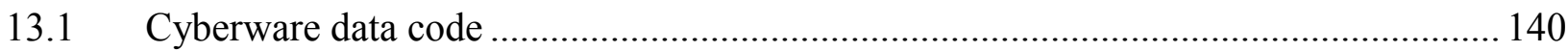

14 Appendix G: TekScan procedure ........................................................................... 152

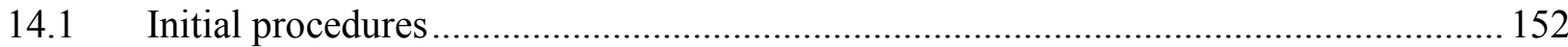

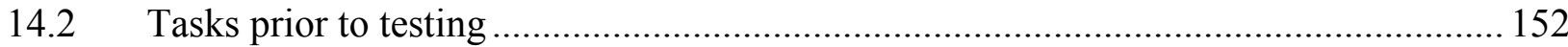

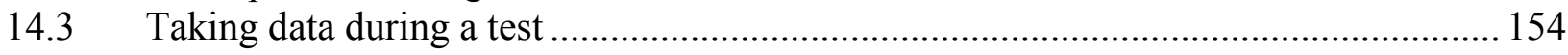

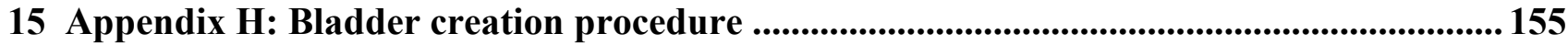

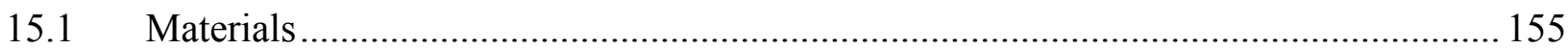

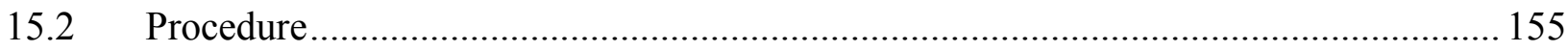

16 Appendix I: Calibration of elastic bands .................................................................. 157

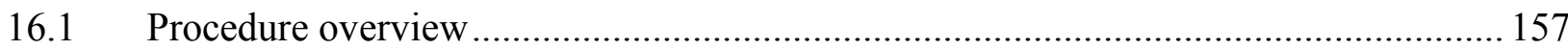

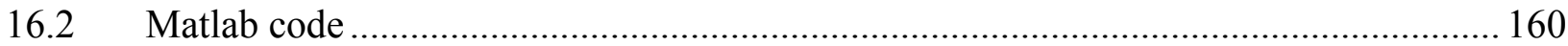

17 Appendix J: Seal between atmosphere and pressure chamber............................................. 167

18 Appendix K: Procedure for making socks ................................................................................ 170

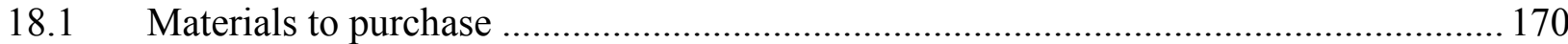

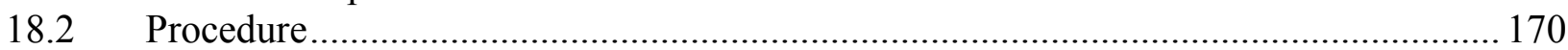

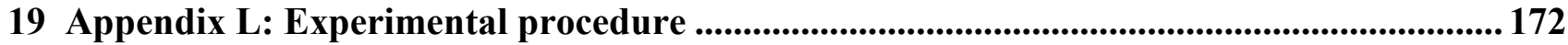

20 Appendix M: A more thorough investigation of Subject 4's test....................................... 176

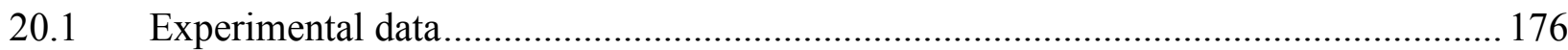

20.2 Subject treatment............................................................................................ 178

21 Appendix N: Matlab code to calculate underpressure felt by subjects in the pilot study .. 180

$21.1 \quad$ Matlab files.......................................................................................... 180

22 Appendix O: A more thorough investigation of Subject 7's test ...................................... 184

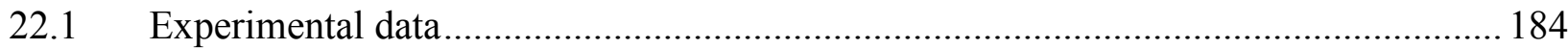

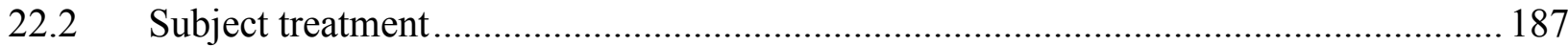

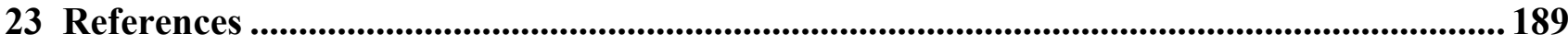




\section{Table of Figures}

Figure 1: Space Activity Suit

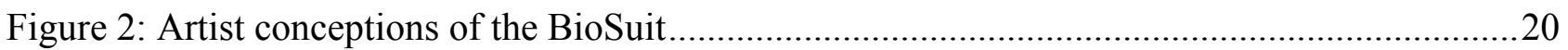

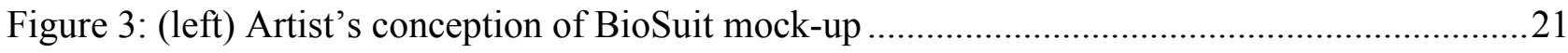

Figure 4: (right) BioSuit mock-up showing LoNE concept ................................................................. 21

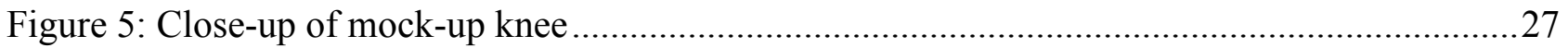

Figure 6: Visual explanation of closure distance requirement......................................................29

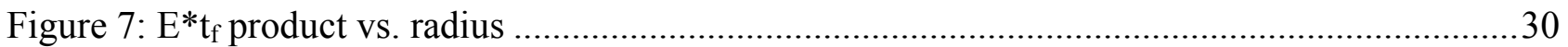

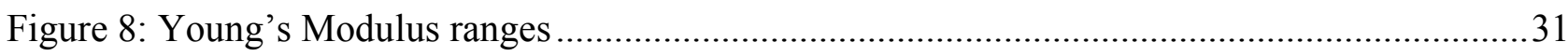

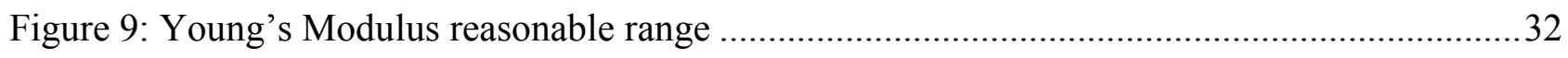

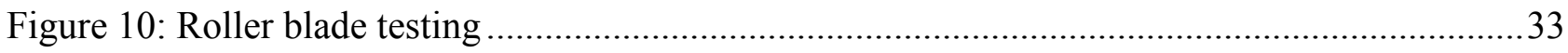

Figure 11: Visible Human Project thigh cross-section...............................................................

Figure 12: Visual explanation for determining Neighbor Normal Method vectors............................40

Figure 13: Method for determining the center of curvature in the 3 Point Circular Method..............41

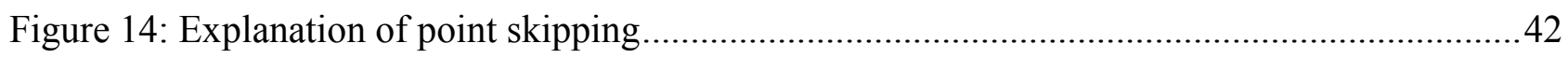

Figure 15: Modeled pressures for Subjects 1-3 in the Wolfrum thesis ............................................43

Figure 16: Modeled pressures for Subjects 4-6 in the Wolfrum thesis ...............................................43

Figure 17: Explanation of numbering system for every cross-section (top view) ………………......44

Figure 18: Modeled pressures for Subjects 1-3 in the Wolfrum thesis ..............................................46

Figure 19: Modeled pressures for Subjects 4-6 in the Wolfrum thesis .............................................46

Figure 20: Smallest cross-section on Wolfrum Subject 6 ………….............................................4

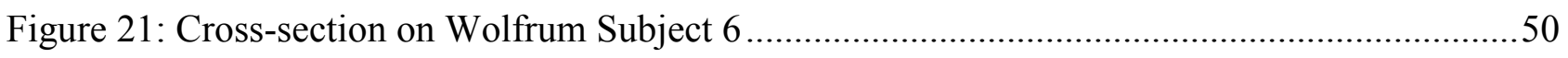

Figure 22: (left) Cross-section skipping 3 points ........................................................................5

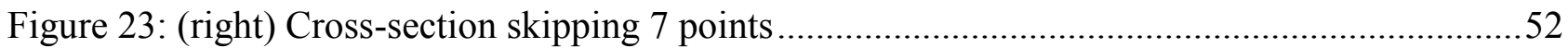

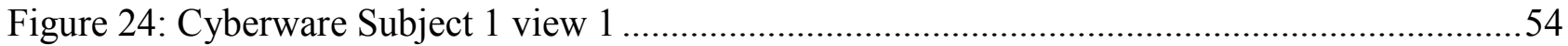

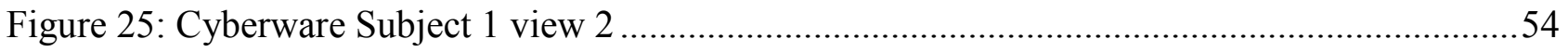

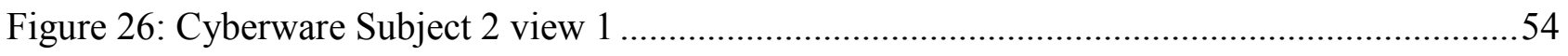

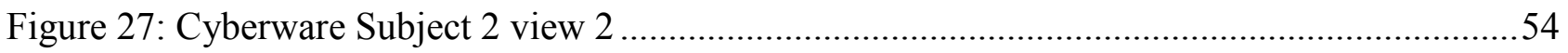

Figure 28: Cyberware Subject 3 view 1 .....................................................................................

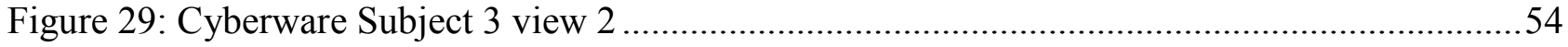

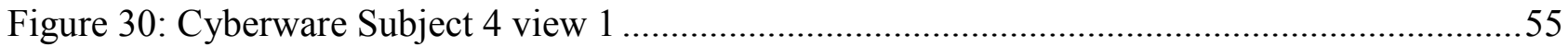

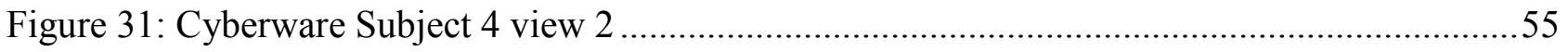




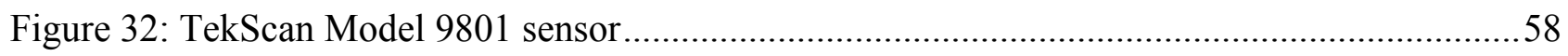

Figure 33: (left) Soft inner cylinder and hard cylinder used for calibration ..................................59

Figure 34: (right) Flat plate calibration device with the plastic bladder and the manometer............59

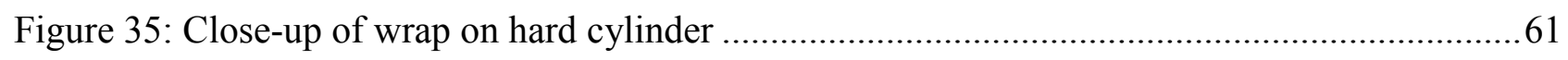

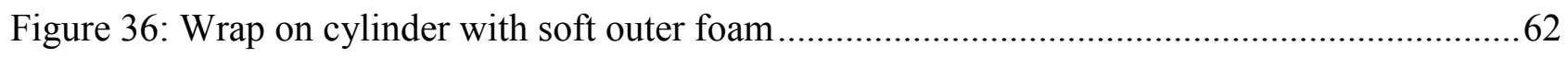

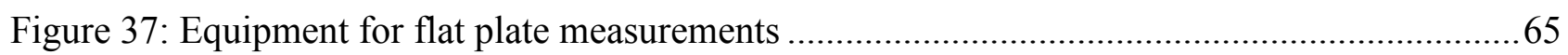

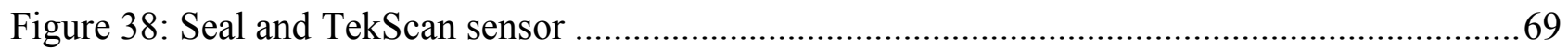

Figure 39: (left) Experimental set-up for human TekScan testing ............................................. 71

Figure 40: (right) Diagram of experimental set-up ........................................................... 71

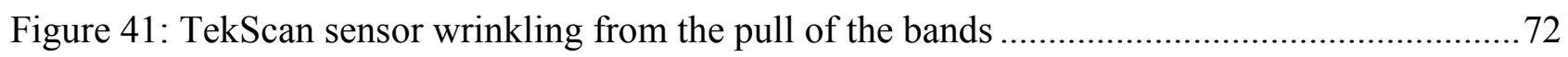

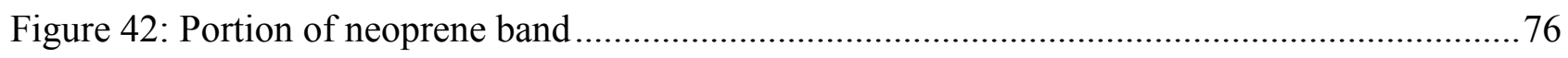

Figure 43: Visual explanation of single wrap and double wrap..............................................76

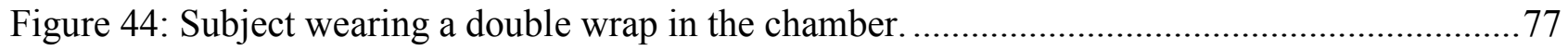

Figure 45: Change in neoprene properties from new to used bands ..........................................8 80

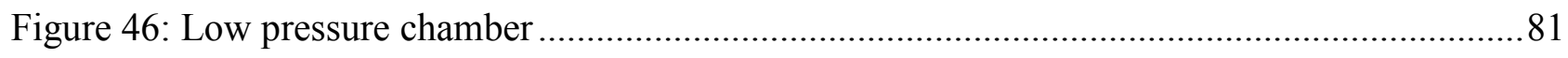

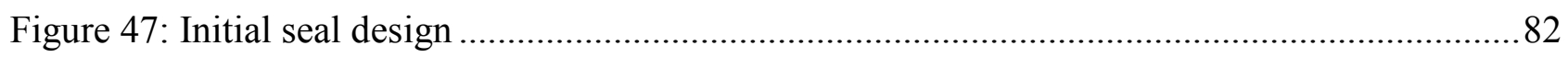

Figure 48 (left): Subject wearing second generation seal design ............................................83

Figure 49 (right): Seal stretched over metal ring to separate pressure chamber from atmosphere

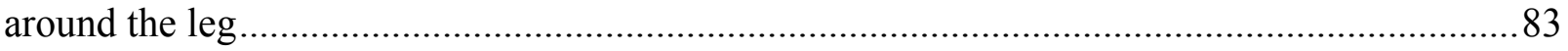

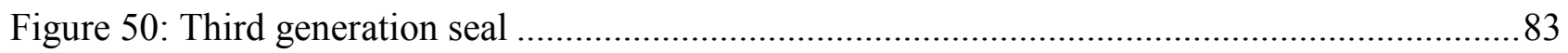

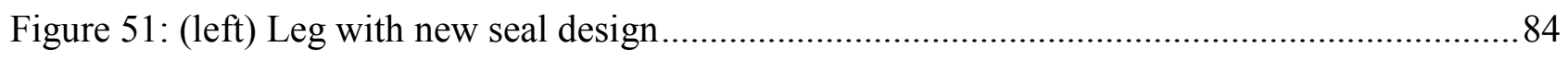

Figure 52: (right) Leg with new seal design and band rolling down the leg................................84

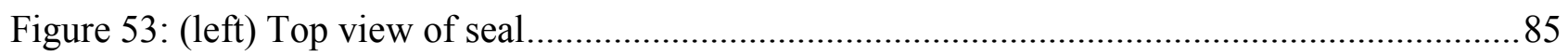

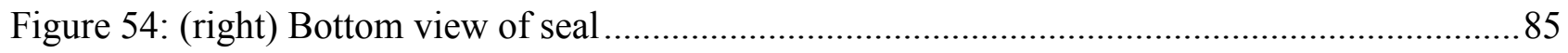

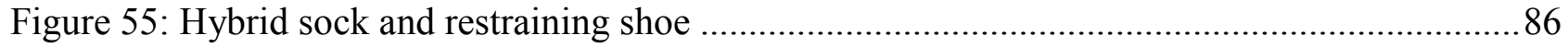

Figure 56: (left) Subject 1 after $32 \mathrm{~min}$ in the pressure chamber...............................................8

Figure 57: (right) Subject 2 after $36 \mathrm{~min}$ in the pressure chamber.............................................89

Figure 58: (left) Skin showing inside the leg wrap in the chamber. ..........................................90

Figure 59: (right) Subject's leg after exiting chamber ........................................................90

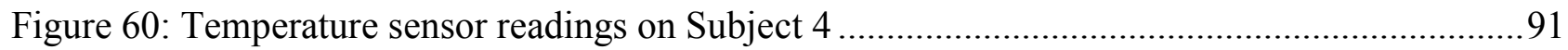

Figure 61: Change in circumference for Subject 1 between bare leg and leg experiencing MCP.....92

Figure 62: Change in circumference for Subject 4 between bare leg and leg experiencing MCP.....93

Figure 63: (left) Subject 1 immediately after exiting the chamber ...........................................95 
Figure 64: (right) Leg of Subject 1 an hour later ................................................................95

Figure 65: (left) Leg of Subject 2 in the chamber, with skin exposed to underpressure..................95

Figure 66: (right) Legs of Subject 2 after the experiment..........................................................95

Figure 68: (left) Leg of Subject 2 in the knee brace.................................................................96

Figure 69: (center) Legs of Subject 2 after the test with the knee brace........................................96

Figure 70: (right) Sock of Subject 2 swelling outside the restraining layer...................................96

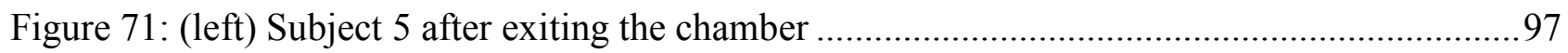

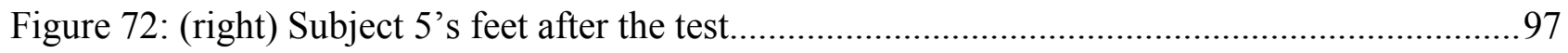

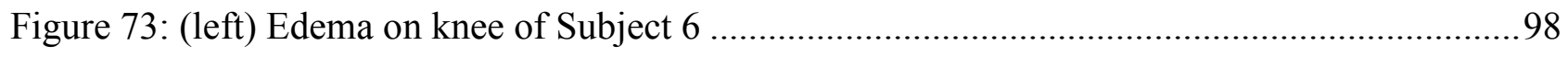

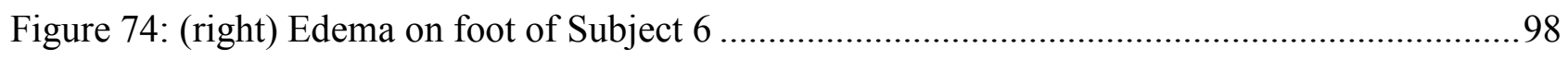

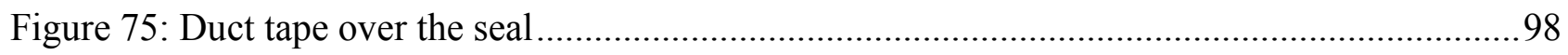

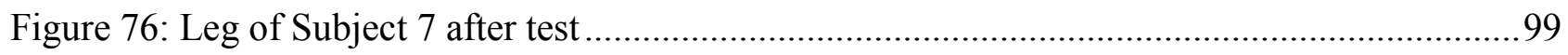

Figure 77: Discomfort measurements by subject and trial .................................................. 100

Figure 78: In-chamber pressure readings from main study................................................ 101

Figure 79: Pressure measurements from subjects under MCP at normal atmospheric pressure...... 101

Figure 80: Change in heart rate for all subjects .................................................................. 102

Figure 81: Change in heart rate for the subjects individually ................................................ 102

Figure 82: Average change in systolic blood pressure ......................................................... 103

Figure 83: Average change in diastolic blood pressure ...................................................... 104

Figure 84: Change in systolic blood pressure for each subject ................................................ 104

Figure 85: Change in diastolic blood pressure for each subject.............................................. 105

Figure 86: Skin temperature change for each sensor based on the averages of the subjects ........... 106

Figure 87: Temperature sensor 1 (right shin anterior) readings ................................................. 106

Figure 88: Temperature sensor 2 (right thigh posterior) readings......................................... 107

Figure 89: Temperature sensor 3 (right thigh anterior) readings ............................................. 107

Figure 90: Temperature sensor 4 (left thigh anterior) readings........................................... 108

Figure 91: Explanation of longitudinal cross-section and change in surface area of skin .............. 119

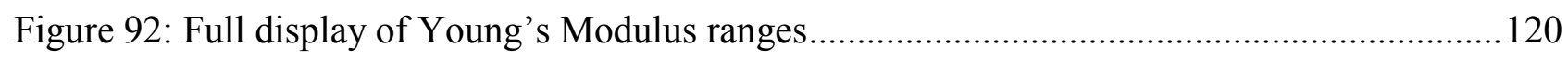

Figure 93: Plastic roll to cover TekScan system ............................................................. 152

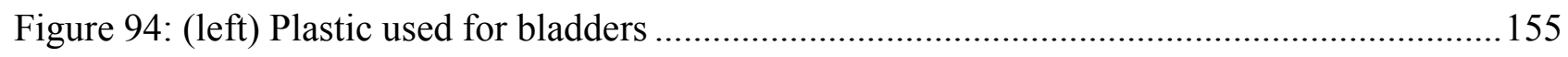

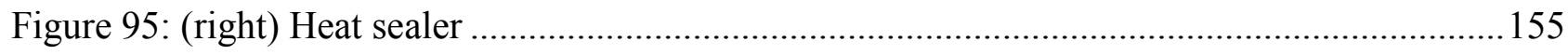

Figure 96: O-rings with bulkhead fitting on bladder........................................................ 156

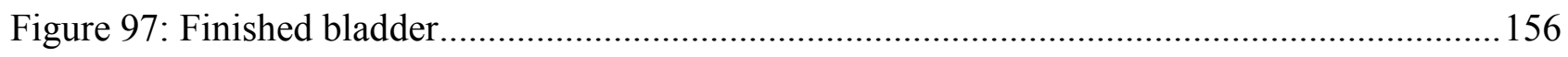




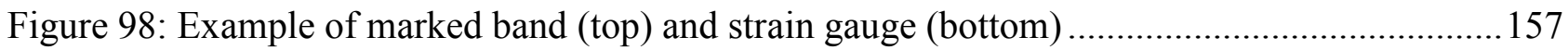

Figure 99: Stress-strain curve for neoprene rubber used for current experiments ....................... 158

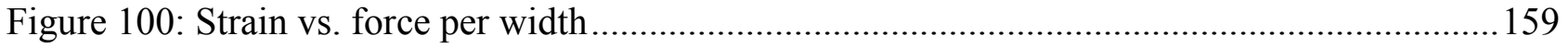

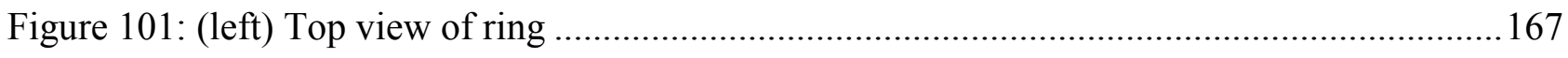

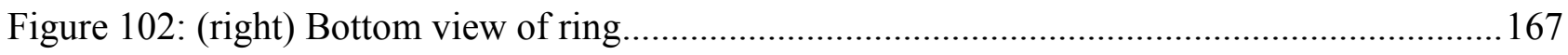

Figure 103: Top ring dimensions, given by ILC Dover ................................................... 168

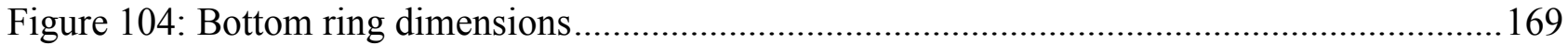

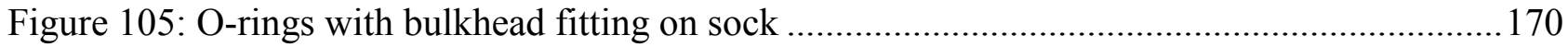

Figure 106: Procedure for sealing socks together ........................................................... 171

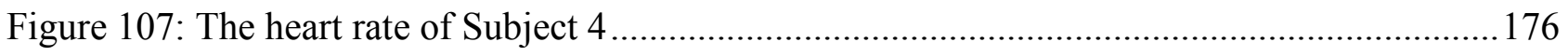

Figure 108: Subject 4's leg after recovery ..................................................................... 178

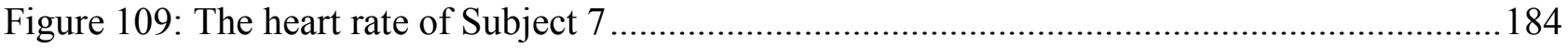

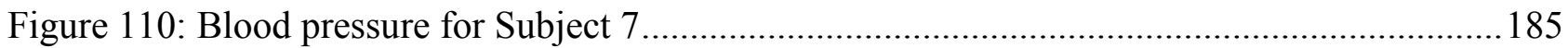

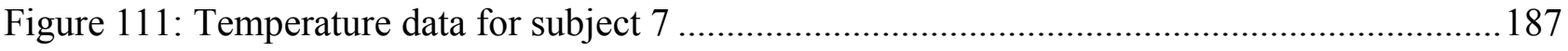




\section{Table of Tables}

Table 1: Functional MCP BioSuit requirements ......................................................................2 24

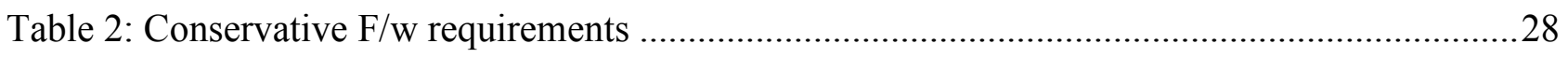

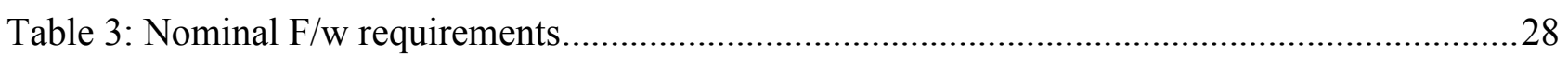

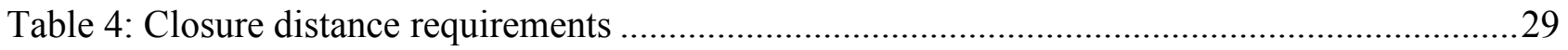

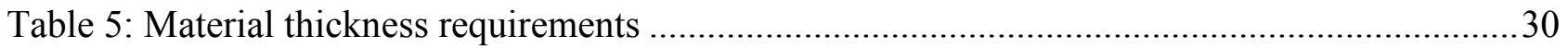

Table 6: Material requirements on Young's Modulus (MPa) ......................................................32

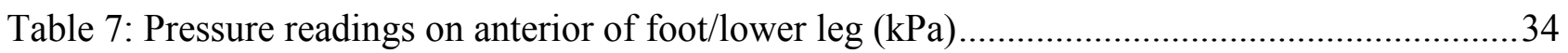

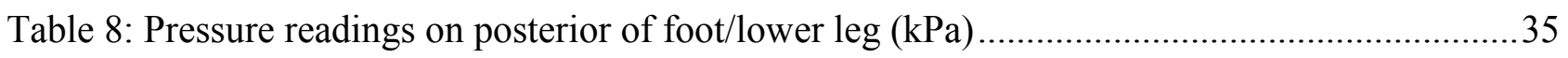

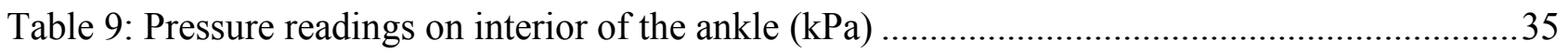

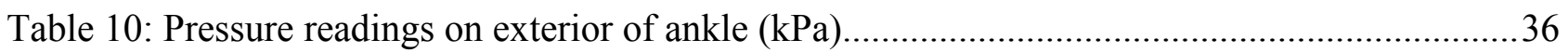

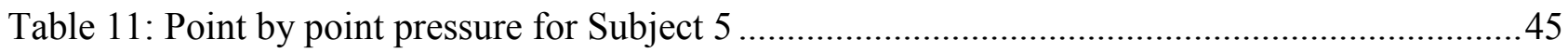

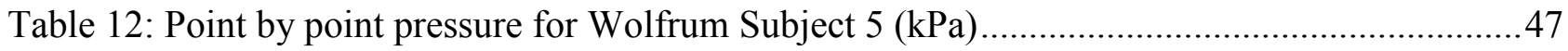

Table 13: Pressure on Wolfrum Subject 5 in Sim experiment [14] .............................................48

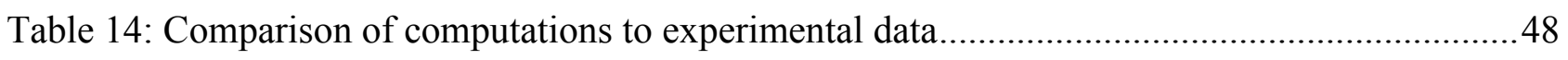

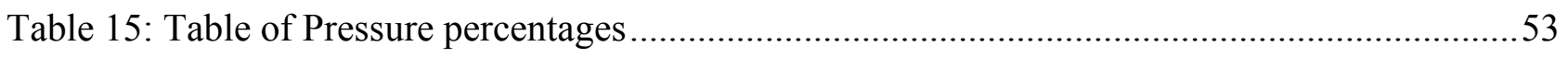

Table 16: Subject 2 pressure readings while experiencing $-225 \mathrm{~mm} \mathrm{Hg}$....................................57

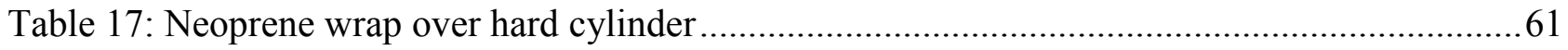

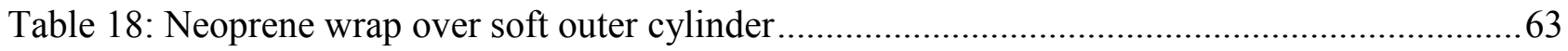

Table 19: Calibrated on soft inner cylinder, measured on soft wrapped cylinder .........................63

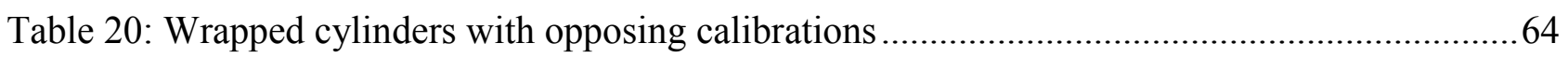

Table 21: Flat plate measurements (calibrated and measured on piece of foam) .........................65

Table 22: Pressure measurements on hard cylinder (calibrated and measured).............................66

Table 23: Pressure measurements on cylinder with soft inner foam (calibrated and measured) .......66

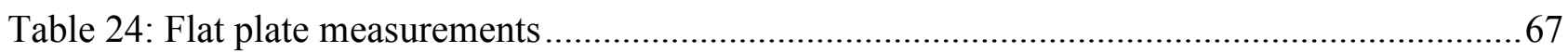

Table 25: Calibrated on hard cylinder, measured on soft cylinder ............................................67

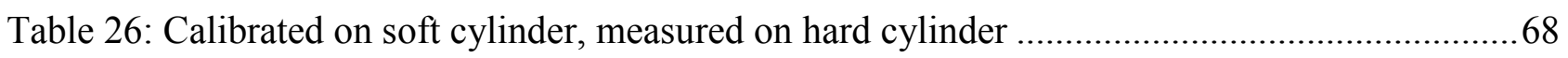

Table 27: Calibrated on flat plate, measured on flat plate with seal ...........................................69

Table 28: Calibrated on soft cylinder, measured on soft cylinder with seal ................................70

Table 29: Calibrated with seal, measured without seal ............................................................. 70

Table 30: Human testing based on hard cylinder calibration....................................................71

Table 31: Human testing based on soft cylinder calibration ................................................. 72 


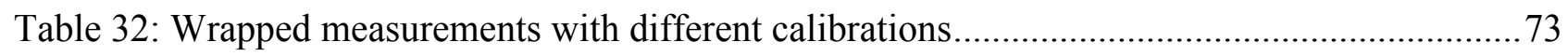

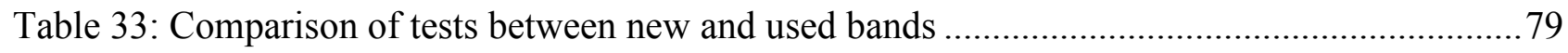

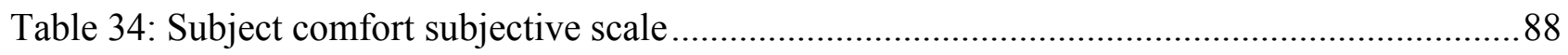

Table 35: Calculated pressure for Subject 4 ....................................................................... 93

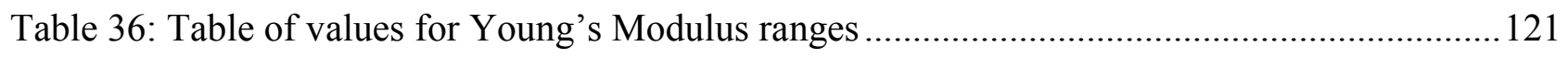

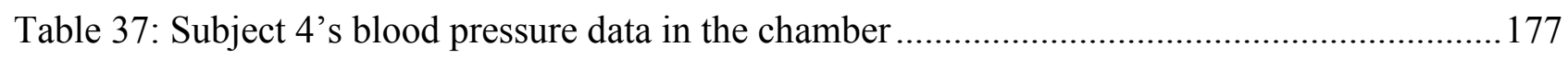

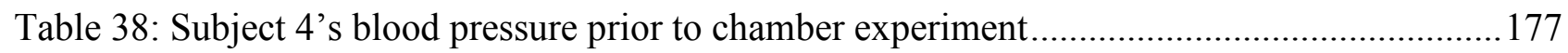

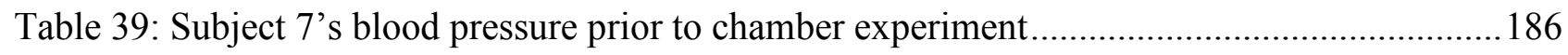




\section{Chapter 1: Introduction}

As part of NASA's Vision for Space Exploration, human explorers shall return to the Moon and then venture to Mars. In order for these missions to conduct exploration and science operations with a minimum of risk, a spacesuit that is superior to the current designs must be developed. This new spacesuit is required to have significantly more mobility than the current design and allow for the safety of astronauts to conduct many extravehicular activities (EVAs) while still being less massive than the current spacesuits.

One option for this new spacesuit design is to use the theory of mechanical counterpressure (MCP). MCP utilizes elastic tension to create pressure directly on the human skin, rather than using gas as a pressurant, as in the current spacesuits. This idea of MCP was first proposed in the 1960's by Webb and Annis [1,2] and other researchers have developed the concept further, although at no point has the design reached operational capability.

MIT is leveraging new technologies and materials as part of the BioSuit system, a spacesuit design based on the principle of MCP. This tight-fitting spacesuit is designed to provide the wearer with the same ease of movement as in normal clothing, and therefore, provide a "second-skin" capability. The BioSuit is a modular spacesuit design, allowing multiple other layers (such as thermal insulation, micrometeroid protection, etc) to be placed above the pressure-producing layer, so that only the minimum number of required layers are worn during any EVA.

This thesis works to further develop the design, by detailing the system requirements, creating a model to understand the best-case scenario of pressure production, and conducting experimental testing using a pressure chamber. The organization of the thesis is as follows:

Chapter 2 serves as a more detailed introduction to MCP designs, with specific emphasis on the BioSuit. It also provides a brief breakdown of the physiological effects of overpressure and underpressure on the human body. A detailed listing of the BioSuit systems level requirements, as well as elastic fabric and closing mechanism requirements is located in this chapter. Finally, an experiment is undertaken using a roller blade to show that the pressure proposed to be produced by the BioSuit can be created relatively easily.

Chapter 3 contains the modeling work accomplished to understand the optimal pressure variation that MCP can produce on the human body. This chapter is split into 2 sections, based on 2 different data sets available. The analysis supports $\mathrm{MCP}$ as a viable replacement to gas-pressurized spacesuits, given the significant percentage of the human body that would receive pressure within an acceptable variation from the desired pressure goal of $30 \mathrm{kPa}$.

Chapter 4 discusses the lengthy testing conducted with the TekScan pressure sensing equipment to understand why the readings from this system have been consistently lower than expected. Tests were conducted in both a flat plate and cylinders composed of various materials, as well as on the human body. The tests prove that a change in the hardness of the calibration surface to the testing surface causes the sensor to read an improper pressure. 
Chapter 5 outlines the design changes made to the experimental hardware, with a special emphasis on the seal between the atmosphere and the pressure chamber used for physiological testing.

Chapter 6 discusses the results from the pilot study of 4 subjects wearing a BioSuit prototype in a chamber pressurized at $-225 \mathrm{~mm} \mathrm{Hg}(-30 \mathrm{kPa})$ below atmospheric pressure. This knowledge was used to conduct a main study with 5 subjects at the same level of underpressure for an hour. Based on blood pressure, skin temperature, heart rate, and qualitative comfort ratings, the bands have successfully protected the full leg, with the exception of the knee. A simple knee brace which filled the concavities of the knee, however, did prove successful in preventing edema.

Chapter 7 succinctly states the conclusions of this thesis, as well as providing proposals for future work. 


\section{Chapter 2: Background}

\subsection{Motivation}

The first space walk in human history was conducted on March 18, 1965 by Alexei Leonov, and since that date the capability for astronauts to exit a spacecraft and conduct Extravehicular Activity (EVA) has been a critical part of human space exploration. Astronauts wearing spacesuits have walked on the Moon, built the International Space Station, and serviced the Hubble Space Telescope. All spacesuits worn in the space environment, from the first design worn by Leonov (the Berkut) to the current spacesuits worn by the Space Station occupants (the Russian-designed Orlan and the American-designed Extravehicular Mobility Unit, or EMU), have utilized gas pressure to protect the wearer from the vacuum of space [3]. While this system has met the basic and critical requirement of keeping the astronaut alive, it still contains many limitations, three of which are of particular concern:

- $\quad$ safety

- flexibility/mobility

- mass

Gas-pressurized spacesuits are considered a safety risk because any puncture, no matter how small, is life-threatening, as it allows life-sustaining oxygen to escape into space. If the astronaut cannot enter a pressurized environment or stop the loss of oxygen rapidly, he or she will suffocate in a short period of time. Therefore, a spacesuit design that exposes a minimum volume of the body to the oxygen necessary for breathing is optimal, as it limits the potential for system failure.

The use of gas as the pressurant limits the flexibility of the suit wearer as the natural "neutral" position of gas-filled spacesuits is a configuration in which all the joints are completely straight. In order to bend their elbows or knees, the astronauts are exerting significant energy to counter the torque of the spacesuit, similar in nature to the constant force needed to hold a balloon in a bent, non-neutral position. The Robotic Space Suit Tester located in the MIT Man Vehicle Lab has measured the torques to be $14.6 \mathrm{~N}-\mathrm{m}$ to bend the knee 72 degrees and $3.7 \mathrm{~N}-\mathrm{m}$ to bend the elbow 80 degrees for a flight class III EMU [4]. Therefore, the astronauts become much more tired as they conduct EVAs than they would accomplishing similar activities normally, which places limits on their ability to explore, perform maintenance, or conduct scientific investigations. Ideally, the spacesuit should provide little resistance to natural human movement such as walking or hammering.

Mass is also a critical issue for the spacesuits, as the current EMU design has a mass of $117.6 \mathrm{~kg}$ (260 lbs) fully loaded in Earth gravity [5]. Since the EMU is designed solely for use in vacuum, the crew must have separate dedicated suits for launch and reentry, which adds to the mass and volume that must be launched on every mission. Exploration on Mars could require separate suits for vacuum and planetary surface EVAs due to the difference in thermal environments, amongst other 
reasons. If one gas-pressurized spacesuit is used, it probably would not be optimal for either environment.

\subsection{Design options}

Given this short analysis of the limitations of gas-pressurized spacesuits, it seems obvious that other design options should be considered. There are two design alternatives available: mechanical counterpressure and a hybrid solution.

Mechanical counterpressure (MCP) was first proposed by Webb and Annis in the 1960's when NASA was still conducting trade studies on which spacesuit design to choose [1,2]. MCP utilizes elastic stretching of material to create pressure directly on the skin, as opposed to the fluid pressure in gas-pressurized spacesuits. This design strategy would solve each of the design limitations mentioned in the previous section.

In a MCP-based design only the head (and perhaps the torso) are exposed to oxygen, so there is significantly less surface area in which punctures can be created than in a gas-pressurized spacesuit. If a hole develops on a MCP garment, an emergency pressure cuff or quick-hardening compound could be used as a temporary stop-gap until a pressurized environment could be reached.

By conforming to the human body, rather than providing a solid "bubble" within which the body must move, a MCP-based design offers a significant improvement in flexibility and mobility, as the user should have the same range of motion as in typical clothing.

A MCP spacesuit would employ a modular design, in which layers such as micrometeroid protection and thermal control could be added or removed to the spacesuit as necessary. This design would remove the need for dedicated spacesuits and reduce the total amount of system mass for spacesuits.

The reason that a MCP spacesuit is not used currently as the spacesuit design is due to the difficulty of producing the required pressure on the human body, especially concavities such as the knee, with a small enough variation in pressure to protect the wearer and still meet realistic donning and doffing timelines $[1,2]$.

A hybrid system uses a fluid layer to produce pressure through MCP on the skin, via an inextensible restraint layer. This design is similar to the concept of a fighter pilot g-suit or a blood pressure cuff. However, this design suffers from the limitations of gas-filled spacesuits, as it still relies on gases to produce pressure without providing the benefits of a purely elastic MCP spacesuit. For this reason, hybrid spacesuits are not an optimal design option and will not garner more than passing attention in this thesis.

\subsection{Literature review (external to MIT)}

Since the inception of the concept of MCP in the 1960's, there has been relatively little further research into a full spacesuit design. This section will provide further details of the Space Activity Suit (SAS) designed by Webb and Annis as well as some information from the more recent research 
conducted in MCP glove design, and an overview of lower body negative pressure (LBNP) and its effects on the human body, to help motivate why the pressure variation on the body is so critical.

\subsubsection{Space Activity Suit (SAS)}

The SAS utilized multiple layers of elastic material to produce pressure, while using a "bubble" helmet to provide oxygen to the suit wearer. Some initial tests were conducted in a vacuum chamber, although none of the experiments lasted longer than 20 minutes. ${ }^{1}$ These experiments showed no signs of swelling due to underpressure, but were not of a long enough time period to show physiological changes that would occur over a typical 8 hour EVA, much less the effects of repeated exposure to vacuum. The design (seen in the figure below) showcased the simplicity of MCP over gas-filled spacesuits, as well as the increases in mobility and safety, but suffered from a dearth of capable materials. The suit required 7 elastic layers since no material could be found with the proper Young's Modulus curve to fit the operational requirements in one layer. Therefore, many layers had to be utilized, and they required 2 assistants and 45-60 minutes to be donned [1,2]. It was for this reason that NASA rejected the MCP-based design, as the additional help for donning and doffing was not available aboard the Mercury, Gemini, or Apollo spacecraft.

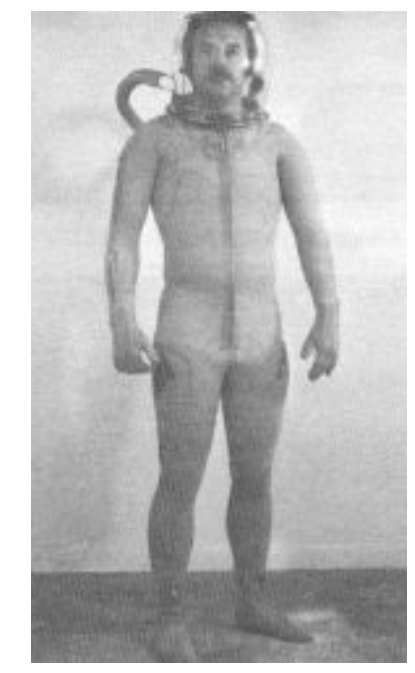

Figure 1: Space Activity Suit

\subsubsection{MCP gloves}

One of the biggest difficulties of EVA work is the lack of sensation astronauts feel in their hands. It is very difficult for them to grasp small devices such as screws needed to complete construction of the ISS, and is therefore a critical impediment to productivity and efficiency. NASA considers this issue important enough that an Astronaut Glove Challenge was part of the Centennial Challenges organized by NASA with $\$ 250,000$ in prize money. Peter Homer won $\$ 200,000$ for his improved

\footnotetext{
${ }^{1}$ This number only includes the time the subject spent at the desired underpressure in the chamber, as there was considerable time spent depressurizing and repressurizing the chamber.
} 
glove design, but the $\$ 50,000$ for the MCP glove was not claimed, as there were no competitors in that category [6].

Due to this interest, most of the MCP work in the past few decades has focused on the hand, rather than a full spacesuit design. In 1983, Clapp designed a MCP glove and tested it in a vacuum chamber. He then compared these results to the A7L-B Skylab-era pressurized glove. The experiments, which lasted 30 minutes each, showed that the MCP glove significantly outperformed the pressurized glove, allowing the subjects to retain $90 \%$ of bare-skin finger mobility, as opposed to $30 \%$ in the pressurized glove. However, the subjects wearing the MCP glove also experienced some edema on their palm, which is another concavity of the body that is difficult to pressurize [7]. While some minor edema after 30 minutes is not a major concern, an astronaut on EVA for 8 hours would experience significantly more, and such a situation could not be tolerated.

Tourbier, Tanaka, et. al, also developed elastic MCP gloves, although it was augmented by an inflatable layer for the dorsum (back of the hand). They succeeded in producing the desired pressure on the dorsum $(27 \mathrm{kPa})$ but only produced $9 \mathrm{kPa}$ on the palm. Despite this low pressure, the glove was successful in preventing increased blood flow, tissue edema, and increase in finger girth that had occurred with a bare hand at underpressure. This group subsequently developed a full elastic sleeve for the arm that produced a higher pressure of $31 \mathrm{kPa}$ at the finger, dorsum, and wrist, but only $21 \mathrm{kPa}$ at the forearm and upper arm. Despite this wide pressure range, again the sleeve prevented adverse changes for underpressures of up to $-20 \mathrm{kPa}$ for 5 minutes $[8,9,10]$.

These studies, whose usefulness is mitigated by the short time period in low pressure environments, donning and doffing limitations, and wide variation in pressure, are still critical for demonstrating that MCP can effectively protect humans from the experience of underpressure. However, significant work must continue to effectively prove MCP as a valid spacesuit design principle.

\subsection{BioSuit}

The BioSuit, a proposal of the Man Vehicle Laboratory at MIT, is designed to fulfill the new requirements described in the first section of this chapter. This MCP-based design is leveraging new technologies and materials not available to previous designers in order to produce a "secondskin" capability for the planetary exploration outlined in the Vision for Space Exploration. The BioSuit is designed to allow the user the same mobility as wearing regular clothing, and is a modular design, in that the suit is composed of many layers, of which the most important is the pressure layer, placed immediately above any undergarments. Ideally, the pressure layer should be

composed of a porous material that would allow the user to sweat. By using the body's natural process, a heavy and complex thermal cooling system, such as that used in the EMU, would be unnecessary, and would dramatically reduce the overall system mass. 

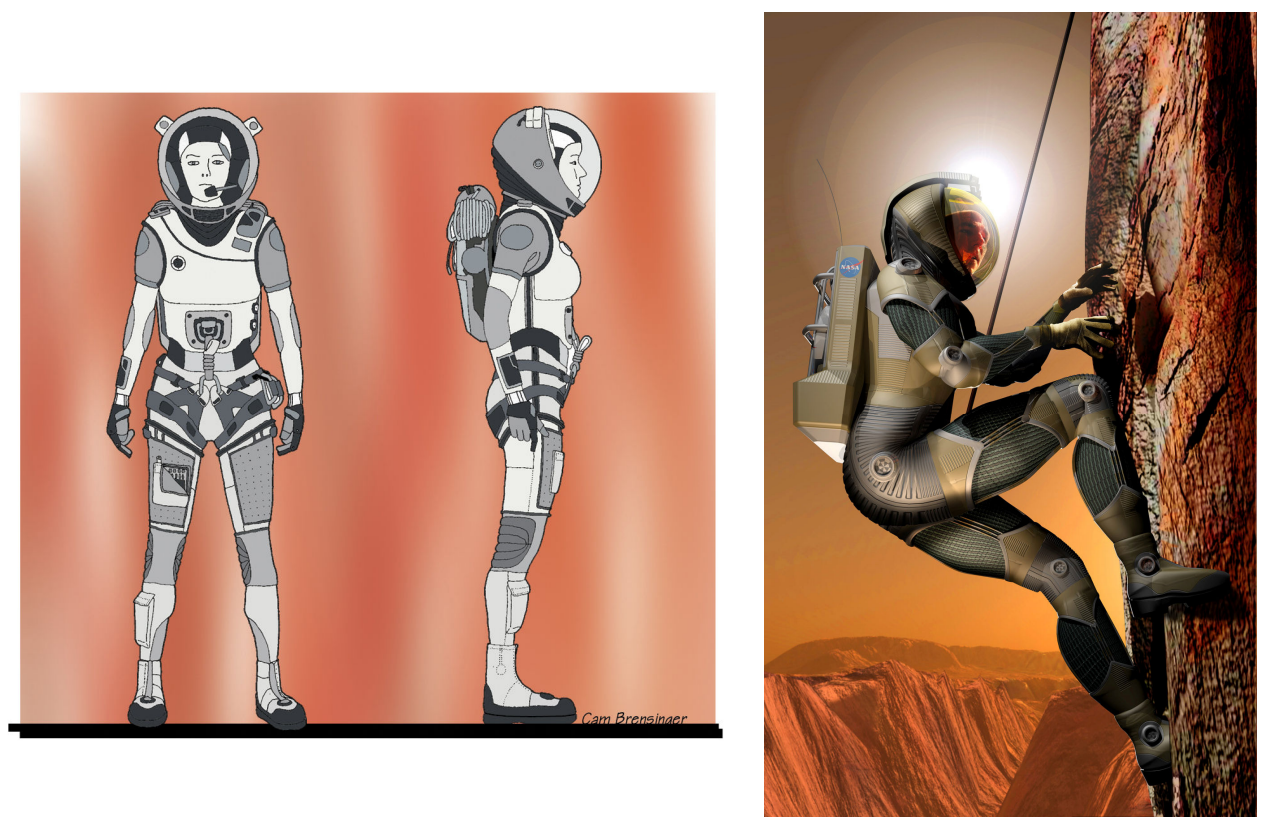

Figure 2: Artist conceptions of the BioSuit ${ }^{2}$

The focus of the work thus far has been in three areas: requirements definition, prototype development and testing, and the physiological effects of MCP on the human body. This thesis expands those topics to a fourth area: theoretical modeling of the capability of MCP, which will be covered in detail in Chapter 3.

\subsubsection{Requirements definition}

The requirements definition is critical as since the suit is designed to provide a "second-skin" capability, so the changes in skin volume, surface area, and shape must be understood. While Bethke and Wolfrum [11,12] have used laser-scanning devices to measure these properties of skin at various knee angles, this thesis builds upon requirements definition in a separate area: defining overall system requirements and material specific requirements, which will be defined later in this chapter.

\subsubsection{Prototype development and testing}

The prototype development and testing is necessary towards developing MCP as a viable alternative to gas-pressurized spacesuits. This work was begun by Pitts, who developed two hybrid designs [13], and continued by both Bethke and Sim [11,14]. Bethke focused on the concept of the Lines of Non-Extension (LoNE) first developed by Iberall as a more flexible inextensible restraint layer for gas-filled spacesuits. The LoNE are empirically measured lines along the body that remain stationary, or nearly stationary, as the suit wearer moves [15]. Very hard materials, such as Kevlar, could be placed along these lines, which would be used to stretch elastic materials to produce

\footnotetext{
${ }^{2}$ Figures courtesy Trotti and Associates, Inc.
} 
pressure. During this past year, a mock-up was made by the Dainese Corporation modeling this design, which can be seen in the figure below.

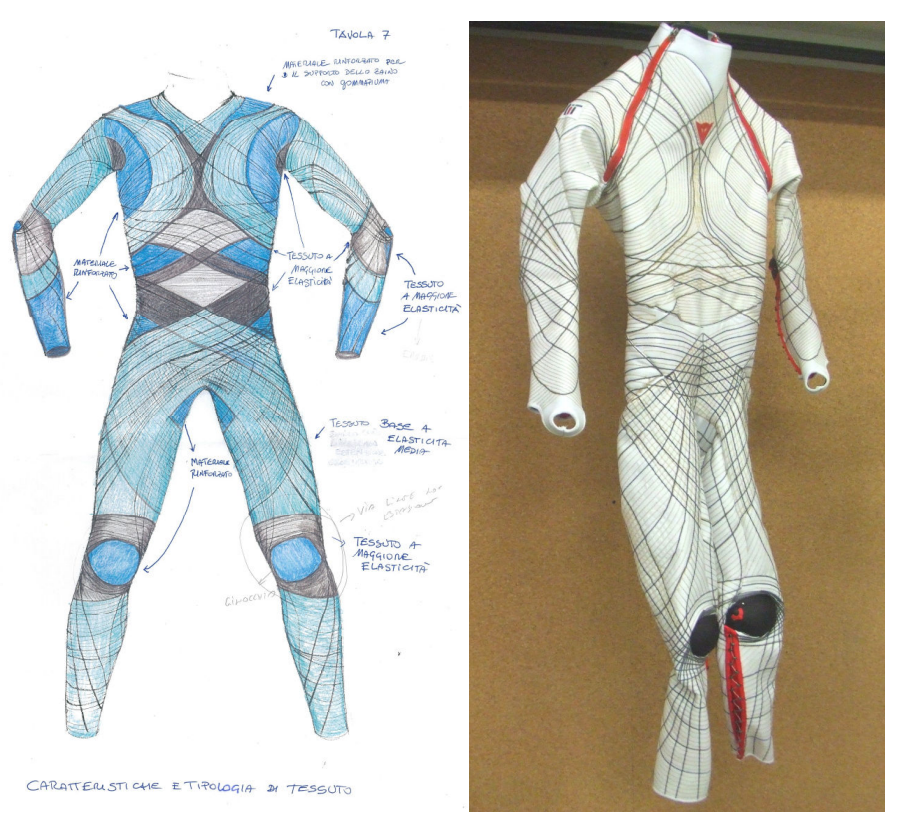

Figure 3: (left) Artist's conception of BioSuit mock-up Figure 4: (right) BioSuit mock-up showing LoNE concept

The second option is to use purely elastic materials to produce pressure. This concept was pursued by Sim, and the prototypes developed as part of this thesis are an extension of his work. ${ }^{3}$ Sim used an elastic bindings concept, in which a long, thin piece of rubber was wrapped in a spiral pattern from the ankle to just below the knee to protect the body from the effects of underpressure. One of the contributions of this thesis is to conduct experiments to discover whether this process can protect the entire leg in a low-pressure chamber. For this elastic approach to work effectively, there must be a mechanism to produce the pressure, or else the design will be quite similar to the SAS, which clearly suffered from donning/doffing limitations.

The proposal for the BioSuit is to use Shape Memory Materials (SMM), which have a "memorized" shape to which they return upon application of heat, pressure, electric current, or other stimuli. The SMM could be attached to the elastic material and contract to a shape in the form of a zipper or some other compact form. The stimulus could force the SMM to expand, and allow the spacesuit to be donned easily. Once the stimulus is removed, the SMM would contract to produce the proper pressure. Similarly, the stimulus to the SMM in the BioSuit could then be added again, and subsequently the spacesuit could be quickly doffed. The Man Vehicle Lab has been working collaboratively with the Midé Corporation, which has extensive experience in SMM. Investigation of these materials is outside the scope of this thesis.

\footnotetext{
${ }^{3}$ It is for this reason that his thesis will be cited quite frequently throughout this thesis.
} 


\subsubsection{Physiological effects of MCP}

The BioSuit team has investigated the physiological effects of MCP. Unpublished work by Carr and Trevino indicate that at least $12 \mathrm{~mm} \mathrm{Hg}(1.6 \mathrm{kPa})$ of pressure variation over a cross-section of the body is tolerable [16]. The Sim experiment had subjects place their bare leg in the low-pressure chamber to experience underpressure and provide a baseline for his suited experiments. In the baseline experiment, in which the pressure was decreased $-25 \mathrm{~mm} \mathrm{Hg}$ every 6 minutes, none of the subjects could tolerate $-225 \mathrm{~mm} \mathrm{Hg}$, and neither of the two women were able to continue past -150 $\mathrm{mm} \mathrm{Hg}$. However, with the elastic bindings to protect them, all the subjects experienced $-225 \mathrm{~mm}$ $\mathrm{Hg}$ for at least 45 minutes, and only mild physiological effects occurred despite a lack of pressure uniformity. Sim theorized that the relative lack of edema may be due to the nature of the calf, which can tolerate more variation in pressure than the arm [14].

The results from the physiological testing in this thesis will add further data to investigate the range of pressures that the users of the BioSuit can tolerate. There has not been a large amount of research in this area, but it is absolutely critical to understand the required pressure variation for a spacesuit design. For this reason, the next section will briefly examine the major concerns of overpressure and underpressure.

\subsection{Further research into localized changes in pressure}

MCP spacesuits must be actively controlled in order to produce the proper pressure. While the design should attempt to keep a constant pressure across the entire body, inevitably the actual pressure produced on the body will vary somewhat from the desired pressure. Both too much pressure (or "overpressure") and too little pressure ("underpressure") can lead to physiological problems.

Both of these conditions (overpressure and underpressure) are of medical interest, although for different reasons. Overpressure is common in hospitals, as bed-ridden patients develop sores from lack of movement, while investigation of underpressure is useful for scientists wishing to understand circulatory mechanisms. However, most of the experimental research in these areas was conducted for such a short time period (less than 10 minutes) or with too high a pressure differential to be of interest for MCP design [14]. Therefore, only the generalized results of this research are useful for this particular application, as further investigation is needed to provide data from MCP research that could be applied to EVA opportunities. ${ }^{4}$

\subsubsection{Overpressure}

With overpressure, too much pressure is directed onto the skin, which can cause inflammation and pressure sores, amongst other injuries. It is important to realize that the creation of sores is inversely proportional to the amount of overpressure. In other words, the larger the force, the more rapidly the sore develops, although long-developing sores (sores caused by a small amount of overpressure over long periods of time) are generally more damaging [17,18,19].

\footnotetext{
${ }^{4}$ Sim devotes an entire chapter in his thesis to these topics, so anyone interested in the details of these experiments can use his list and summaries as a starting point for further reading.
} 
The production of sores is dependent on the shear strain and friction against the skin. As these factors increase in intensity, the amount of pressure needed to cause sores is reduced. Additional factors that effect the production of pressure sores include humidity and lack of evaporation. It is thought that sores begin internally near the bone, and then rise from the bone to the skin, [18] so special attention should be paid to overpressure on areas of the body such as the shin or elbow, which are particularly bony.

The MCP suit wearer will experience overpressure over the entire body before entering a vacuum or low pressure environment, and adverse physiological effects may occur if the time between donning and EVA is more than 20-30 minutes [1,2]. However, the critical concern is localized overpressure, rather than full body overpressure, as it is the former that causes pressure sores.

\subsubsection{Underpressure}

When a subject experiences undepressure, the fluids in the body redistribute to areas of lower pressure, similar to the manner in which gases naturally disperses to equalize pressure. The pooling of the blood in a particular area causes swelling as the tissue expands to hold the additional fluid. This swelling, which additionally turns the skin red, is thought to be more tolerable in the lower body as it is farther from the heart. Additionally, the rushing of blood away from the head and heart can cause syncope (fainting) which could easily be fatal in a space environment, unless that astronaut is taken to a pressurized environment quickly [20].

\subsubsection{Discussion}

For these reasons, it is critical to measure the pressure variation caused by a MCP spacesuit design. However, the requirements that this suit must meet are not yet defined. Research needs to be conducted into relationships between time and pressure variations that cause both sores and edema, and factors such as humidity, shear strain, and friction would have to be carefully controlled. Another key requirement that needs to be established is how much skin surface area can be exposed to overpressure or underpressure before damage occurs. Until this research is conducted, the design can only have a target for pressure variation, not a requirement, and this is a major obstacle for analyzing whether MCP is a valid alternative to the current gas-filled spacesuits.

\subsection{System-level requirements}

Many systems have failed because the designers have failed to adequately understand their requirements. While the BioSuit does not have a pressure variation requirement, it is important to detail each of the system level requirements, as well as the fabric requirements for pressure production.

This table outlines each of the requirements for the BioSuit, with explanations for each of the requirements in the section below. Some of this work had been defined previously, but these values had not been organized in a centralized table (see Table 1). 
Table 1: Functional MCP BioSuit requirements

\begin{tabular}{|c|c|}
\hline Parameter & Value \\
\hline \multicolumn{2}{|c|}{ Performance } \\
\hline Range of radii to be protected & Radius: $2.2 \mathrm{~cm}-10.7 \mathrm{~cm}$ \\
\hline Pressure to be produced & Min: $25 \mathrm{kPa}$, Target: $30 \mathrm{kPa}$ \\
\hline Acceptable variation of MCP & $\pm 12 \mathrm{~mm} \mathrm{Hg}(1.6 \mathrm{kPa})$ uncertain - see text \\
\hline Actuation speed & $<10 \mathrm{~min}$ \\
\hline Lifetime (cycles) & 10,000 \\
\hline \multicolumn{2}{|c|}{ Environment } \\
\hline Thermal environment (in vehicle) & Room temperature $\left(21-23{ }^{\circ} \mathrm{C}\right)$ \\
\hline Thermal environment (EVA) & Moon: $-233-123{ }^{\circ} \mathrm{C}$, Mars: $-123-17^{\circ} \mathrm{C}$ \\
\hline Gas/fluid compatibility & air-permeable fabric \\
\hline External pressure $(\min / \max )$ & Min: vacuum, max: $0.6 \mathrm{kPa}$ \\
\hline $\begin{array}{l}\text { Max. continuous skin area exposed to } \\
\text { vacuum }\end{array}$ & $<1 \mathrm{~mm}^{2}$ \\
\hline \multicolumn{2}{|c|}{ Human Factors } \\
\hline Donning procedure & Completed by one person, $<10 \mathrm{~min}$ \\
\hline $\begin{array}{l}\text { Mobility: } \\
\text { elasticity once donned }\end{array}$ & $\begin{array}{l}\text { a) }<7 \mathrm{~N}-\mathrm{m} \text { additional work to flex knee } 72^{\circ} \\
\text { b) Permit wearer to bend knee }>90^{\circ} \\
\text { c) }<1.5 \mathrm{~N} \text {-m to bend the elbow } 80^{\circ}\end{array}$ \\
\hline Thickness & $<5 \mathrm{~mm}$ \\
\hline
\end{tabular}

\subsection{Rationale for requirements}

\subsubsection{Range of radii to be protected}

The radius of the limbs on humans ranges from $2.2 \mathrm{~cm}$ on the wrist of a $5^{\text {th }}$ percentile female to 10.7 $\mathrm{cm}$ on the upper thigh of a $95^{\text {th }}$ percentile male [21]. These values are based solely on human limbs, and do not include the torso.

\subsubsection{Pressure to be produced}

The partial pressure of oxygen that humans breathe is $21 \mathrm{kPa}$, so in order to provide constant pressure across the body, this is the absolute minimum pressure that must be provided. The requirement of $25 \mathrm{kPa}$ provides a margin over this minimum value. The more pressure produced 
via the MCP the better (up to normal atmospheric pressure), since pre-breathing procedures could be shortened and EVA would become a more efficient activity. The goal of this project is to demonstrate mechanical counterpressure at $30 \mathrm{kPa}$ because of these benefits.

\subsubsection{Acceptable variation of MCP}

This requirement has a lot of uncertainty since pressure variability around the circumference of a human limb is not a normal medical condition, so there is a lack of data in the literature about its effects. It was determined through testing that $12 \mathrm{~mm} \mathrm{Hg}$ of pressure variation proved unlikely to cause edema, but that does not necessarily mean pressure differentials above $12 \mathrm{~mm} \mathrm{Hg}$ will cause edema. Some limited research into this subject indicates that up to $20 \mathrm{~mm} \mathrm{Hg}$ pressure variation will not cause edema either. More testing is necessary to find the proper acceptable pressure variation, and until that is undertaken, the more conservative value will serve as the requirement [16].

\subsubsection{Actuation speed}

For periods of time longer than 10 minutes, the wearer of a MCP garment begins to experience significant discomfort due to overpressure. Therefore, the actuation speed must be as rapid as possible. This actuation speed is for the entire body, since all parts of the body should be at the same pressure [14].

\subsubsection{Lifetime cycles of BioSuit}

While it is highly unlikely that the BioSuit will be worn 10,000 times, the number of cycles is assumed to include significant testing.

\subsubsection{Thermal environment (in vehicle)}

The vehicles in which the astronauts will be traveling are assumed to operate at room temperature for maximum comfort $\left(21-23{ }^{\circ} \mathrm{C}\right)$.

\subsubsection{Thermal environment (EVA)}

The temperatures that astronauts would face ranges from -233 to $123{ }^{\circ} \mathrm{C}$ on the Moon and from -123 to $17^{\circ} \mathrm{C}$ on Mars. Possible locations of Moon bases include not just the equator, but also the South Pole, which becomes very cold during the 180-day night. The Martian temperature values are from the equator, which is likely to be the landing site.

It is assumed that other layers will be placed above the BioSuit MCP material, so that the chosen material will not be subjected directly to this large temperature range [22]. 


\subsubsection{Gas/fluid compatibility}

For thermal considerations, in order to simplify the BioSuit and provide maximum comfort to the wearer, the suit will be air-permeable and allow perspiration to be wicked away into the atmosphere.

\subsubsection{External pressure (min/max)}

The Moon has no atmosphere, while Mars has an average pressure of $0.6 \mathrm{kPa}$, although this pressure varies with elevation and with the seasons. The BioSuit must be designed to work in each of these environments.

\subsubsection{Skin area exposed to vacuum}

No harmful effects occur when the maximum extent of continuous skin exposure is less than $1 \mathrm{~mm}^{2}$. The exposed skin area is assumed to be the pore size of the suit material, and areas this size exposed to vacuum all over the body, although it is unclear how close together these pores can be placed without causing edema. The openings of the SAS suit designed by Webb had pore sizes on average of between $0.5 \mathrm{~mm}^{2}$ and $1 \mathrm{~mm}^{2}$ when under tension [1,2].

If a hole develops which is larger than $1 \mathrm{~mm}^{2}$, the astronaut still has plenty of time to reach a pressurized environment because the damage is localized, and there is no loss of oxygen in a MCP suit. However, the material should not be easily broken for safety concerns. If the material is broken, it should also not allow the hole to expand quickly, otherwise the astronaut could have significant skin area subject to vacuum (or near-vacuum) $[1,2]$.

\subsubsection{Donning procedure}

Typical explorations on a Moon or Mars mission may involve only 2 astronauts. It would be impractical to expect that a third person will always be available to help with suit donning and doffing during off-base excursions. Therefore, the donning and doffing process should be capable of being completed alone, and in less than 10 minutes.

\subsubsection{Donned mobility (elasticity)}

a) The EMU requires an additional $14.6 \mathrm{~N}-\mathrm{m}$ of torque than would be done unsuited to bend the knee to $72^{\circ}$. The new requirement is not based on any physiological value, but to reduce by half (approximately) the additional torque created by the EMU to bend the knee $72^{\circ}$ [4].

b) The EMU significantly limits the leg motion of the user, and past spacesuits have not had full range of motion either. For astronauts on Mars, activities including squatting, running, rapelling down cliffs, etc. necessitate significant mobility to complete mission objectives, and the BioSuit must be able to provide this level of mobility. 
c) The BioSuit is designed to improve the mobility of the astronauts when compared to the EMU. Therefore, it should allow the astronauts to move their elbows without producing as much torque as in the EMU.

\subsubsection{Thickness of material}

To keep the envelope around the astronaut as small as possible, the thickness of the material shall not exceed $5 \mathrm{~mm}$, as further material would limit mobility.

\subsection{Material requirements}

There are two main components of the BioSuit using the pure elastic design, without the lines of non-extension: the fabric that will be worn on the body, and the closure mechanism that will produce the pressure. No final decision has been made on the closure mechanism (which will be discussed further in later sections), but the following figure (a close-up picture of the knee of the Dainese mockup) will be used to illustrate one potential solution.

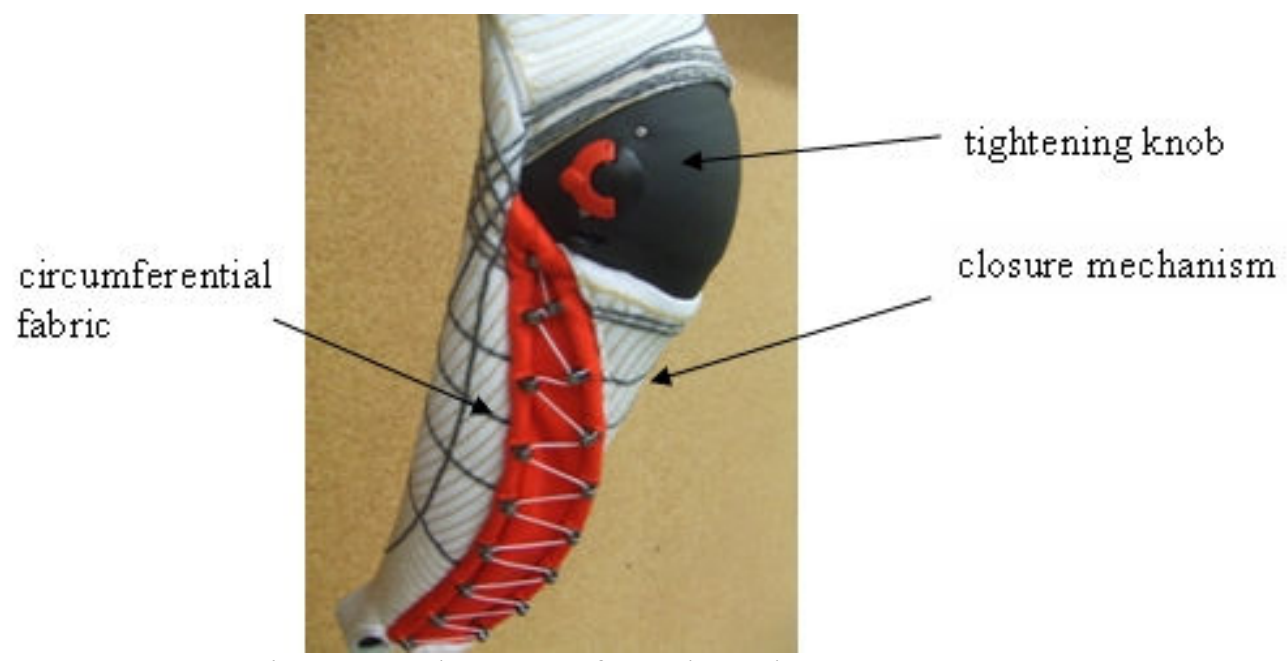

Figure 5: Close-up of mock-up knee

In this example, the knob on the kneecap pulls on the thread, causing the large zigzag gaps to decrease significantly, thereby creating pressure on the wearer. The material requirements are split by the fabric requirements and the closure mechanism requirements. The latter requirements shall be dealt with first.

\subsubsection{Force/width requirements}

From NASA-STD-3000, the radius of a $5^{\text {th }}$ percentile small woman's wrist is $0.022 \mathrm{~m}$. The radius of the thigh of a $95^{\text {th }}$ percentile large man is $0.107 \mathrm{~m}$. These values are taken as the limits of the semi-cylindrical human limb sections on which MCP must be created.

Given the equation that: 


$$
\begin{aligned}
& \frac{F}{w}=P \times r \\
& \mathrm{~F}=\text { force in tension, } \mathrm{N} \\
& \mathrm{P}=\text { pressure, } \mathrm{Pa} \\
& \mathrm{r}=\text { radius of body, } \mathrm{m} \\
& \mathrm{W}=\text { width of the material, } \mathrm{m}
\end{aligned}
$$

and the desired pressure to be $30 \mathrm{kPa}$, it is easy to show that the minimum and maximum forces per unit width are:

Table 2: Conservative $\mathrm{F} / \mathrm{w}$ requirements

\begin{tabular}{|c|c|}
\hline Minimum force/width & Maximum force/width \\
\hline $6.54 \mathrm{~N} / \mathrm{cm}$ & $32.19 \mathrm{~N} / \mathrm{cm}$ \\
\hline
\end{tabular}

However, it is unlikely that the astronaut corps would fit these extreme values. A more nominal set of radius ranges $(0.03 \mathrm{~m}$ minimum to $0.09 \mathrm{~m}$ maximum) would yield the following target region for the elastic force that must be created by whatever material is chosen for a MCP suit:

Table 3: Nominal F/w requirements

\begin{tabular}{|c|c|}
\hline Minimum force/width & Maximum force/width \\
\hline $9 \mathrm{~N} / \mathrm{cm}$ & $27 \mathrm{~N} / \mathrm{cm}$ \\
\hline
\end{tabular}

\subsubsection{Closure distance requirements}

The Shape Memory Material (SMM) used to create the pressure still requires significant research, and cannot provide unlimited strain capabilities. To that end, this requirement setting will limit the distance the SMM must close to a maximum of $5 \mathrm{~cm}$. With a larger closure distance, the force exerted on the material by the closure mechanism would not be pulling together across one plane of the leg as in the drawing on the right in Figure 6. Instead the forces are pulling in perpendicular directions, as in the drawing on the left in Figure 6. This causes the closure mechanism to do significantly more work and is unrealistic. 


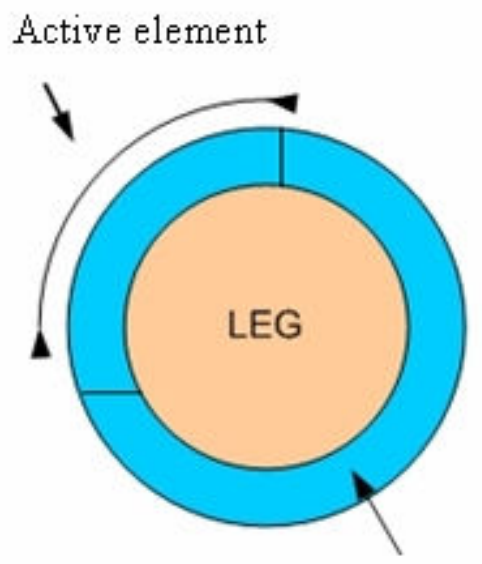

Elastic material
Active element

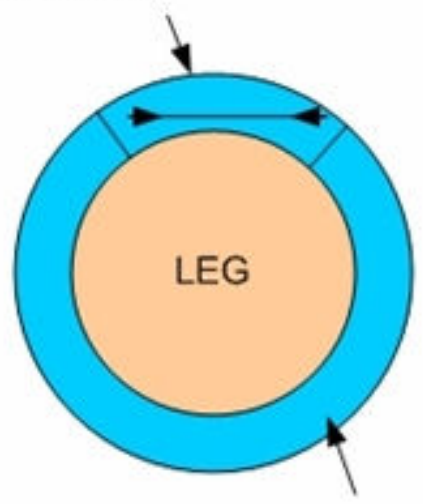

Elastic material

Figure 6: Visual explanation of closure distance requirement

A minimum value must also be set, since pulling the elastic material taut over such a small distance would provide little force. The following table outlines the requirements.

Table 4: Closure distance requirements

\begin{tabular}{|c|c|}
\hline Minimum closure distance $(\mathrm{cm})$ & Maximum closure distance $(\mathrm{cm})$ \\
\hline 1 & 5 \\
\hline
\end{tabular}

In addition to the closure mechanism requirements, there must be requirements placed on the fabric as well.

\subsubsection{Fabric Requirements}

The key equation for this section is:

(Equation 2)

where,

$$
E \times t_{f}=\frac{P \times r}{\varepsilon}
$$

$$
\mathrm{E}=\text { Young's Modulus, } \mathrm{Pa}
$$

$\mathrm{t}_{\mathrm{f}}=$ thickness of the material, $\mathrm{m}$

$\mathcal{E}=$ strain, unitless

In this equation (a derivation of which is available in Appendix A) the right-hand side is known, while the two terms on the left-hand side are variable. The possible ranges for both the Young's Modulus and material thickness shall be identified in this section. It is important to note that the values for the Young's Modulus are only relevant for producing circumferential pressure on the human body. The longitudinal Young's Modulus should be much lower (more flexible) so that the 
material will not produce any unwanted torque over the knee. ${ }^{5}$ In the rest of this section, only the circumferential properties of the fabric are analyzed.

First, the graph identifying the desired product of $\mathrm{E}^{*} \mathrm{t}_{\mathrm{f}}$ shall be displayed, compared to the radius of the subject. Each curve represents a different closure distance accomplished by the SMP. Note that the $1 \mathrm{~cm}$ closure has the highest product, which makes sense, since this distance causes the smallest strain, while $\mathrm{P}$ and $\mathrm{r}$ are constant.

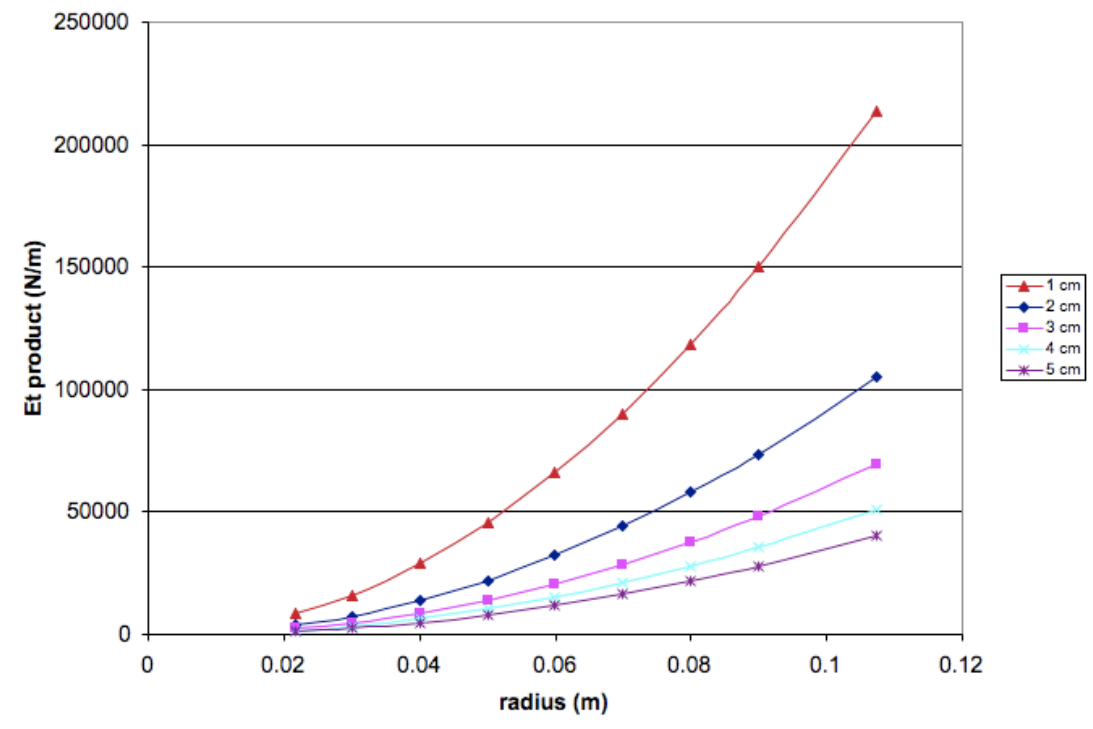

Figure 7: $\mathrm{E}^{*} \mathrm{t}_{\mathrm{f}}$ product vs. radius

However, this graph is rather unwieldy, since $\mathrm{E}^{*} \mathrm{t}_{\mathrm{f}}$ is not a common product on which materials are designed. While the BioSuit has no system-level requirements on the Young's Modulus, it does have a maximum requirement for thickness $(5 \mathrm{~mm})$, as otherwise the suit would be too bulky. Choosing a minimum of $0.5 \mathrm{~mm}$ for the thickness (otherwise the material may break too easily) leads to the following range of requirements for Young's Modulus.

Table 5: Material thickness requirements

\begin{tabular}{|c|c|}
\hline Minimum thickness $(\mathrm{mm})$ & Maximum thickness $(\mathrm{mm})$ \\
\hline 0.5 & 5 \\
\hline
\end{tabular}

The same colored lines represent the high Young's modulus (minimum thickness) and the low Young's Modulus (maximum final thickness). In other words, given a specific closure distance, and the thickness requirements, the Young's Modulus must fall between these two curves. The

\footnotetext{
${ }^{5}$ Appendix B will analyze the needed Young's Modulus to produce pressure over the knee using longitudinal strains, although this is hardly ideal.
} 
following figure represents a reasonable limitation on the Young's Modulus, as the full trade space is seen in Appendix C. ${ }^{6}$

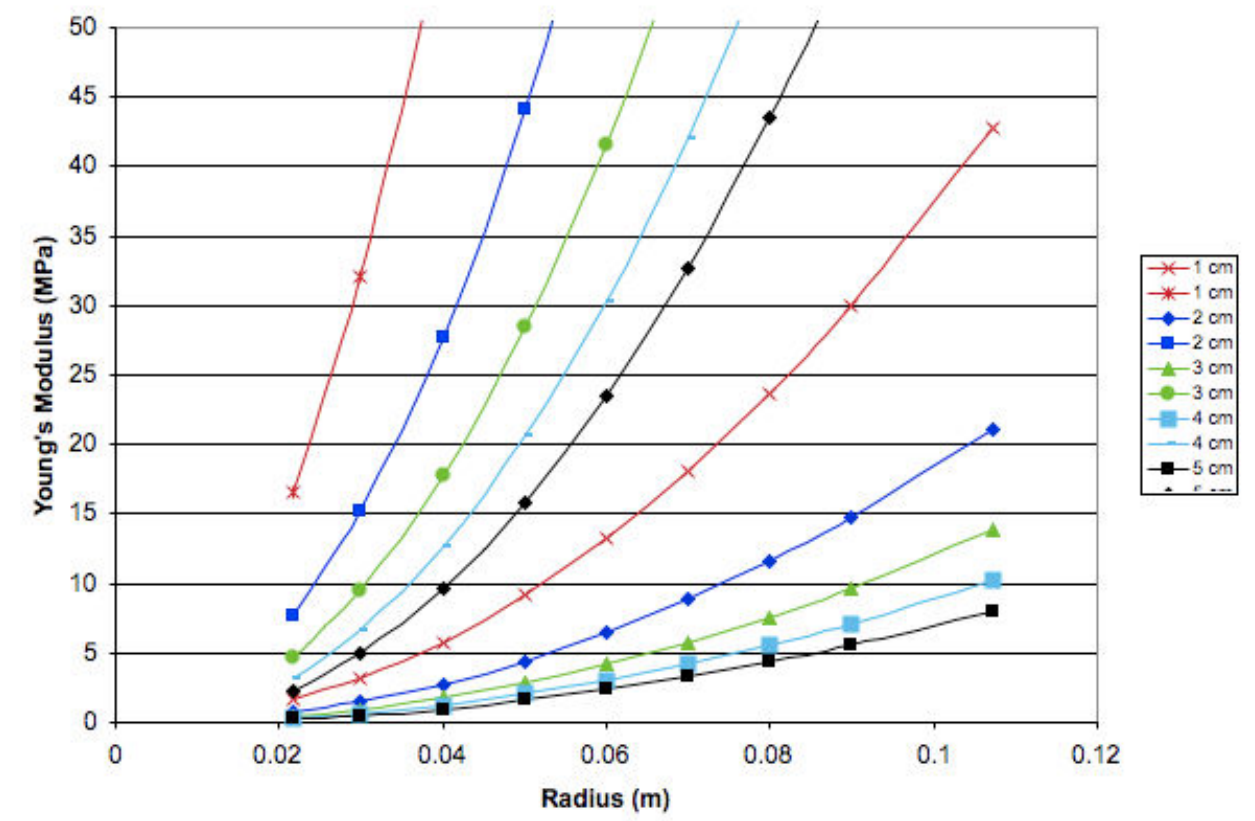

Figure 8: Young's Modulus ranges

This figure contains a large number of lines however, and is somewhat difficult to read. The most important lines are those that band the range of acceptable values. As noted before, the largest Young's Modulus for a cross-section will be based on selecting the minimum thickness, since the two factors vary inversely, as seen in Equation 2. Similarly, the smallest value for the Young's Modulus will be chosen based on the maximum thickness. The following figure displays two lines that are the outer limits of a reasonable limitation on the Young's Modulus given these thickness parameters and the selection of a desired pressure of $30 \mathrm{kPa}$. Any combination of the Young's Modulus and thickness that fits between these lines is acceptable.

\footnotetext{
${ }^{6}$ The Matlab code used to create these requirements is available in Appendix D.
} 


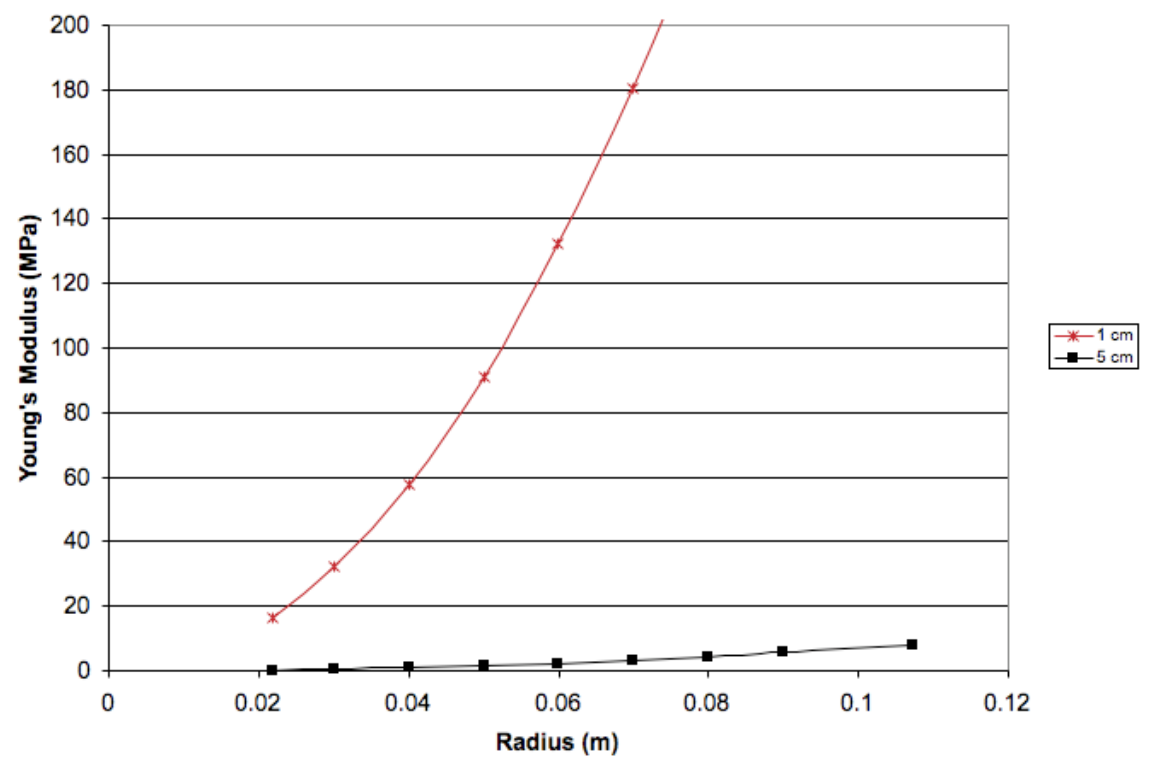

Figure 9: Young's Modulus reasonable range

The same data shown above is displayed in table form:

Table 6: Material requirements on Young's Modulus (MPa)

\begin{tabular}{|c|c|c|}
\hline Radius $(\mathrm{m})$ & $\begin{array}{c}\text { E max }(\mathrm{t}=0.5 \mathrm{~mm}) \\
\mathrm{MPa}\end{array}$ & $\begin{array}{c}\text { E low }(\mathrm{t}=5 \mathrm{~mm}) \\
\mathrm{MPa}\end{array}$ \\
\hline 0.022 & 16.61 & 0.23 \\
\hline 0.03 & 32.13 & 0.50 \\
\hline 0.04 & 57.92 & 0.97 \\
\hline 0.05 & 91.25 & 1.58 \\
\hline 0.06 & 132.12 & 2.35 \\
\hline 0.07 & 180.53 & 3.27 \\
\hline 0.08 & 236.47 & 4.35 \\
\hline 0.09 & 299.96 & 5.57 \\
\hline 0.107 & 427.60 & 8.04 \\
\hline
\end{tabular}

Each row in the table corresponds to a particular average cross-sectional radius. For example, in order to produce the proper pressure over a cross-section of the body with an average radius of 2.2 $\mathrm{cm}$, the Young's Modulus must range between 0.2 $\mathrm{MPa}$ to 16.6 MPa. It is important to understand from this table that any Young's Modulus between $8.0 \mathrm{MPa}$ and $16.6 \mathrm{MPa}$ is acceptable for every reasonable cross-sectional radius of curvature. Therefore, a material with a Young's Modulus in that range over the desired strain values should be chosen to simplify the design, otherwise multiple materials may be needed. 


\subsection{Proof of concept}

This chapter has thus far expanded upon a number of topics, but one missing aspect is the answer to a rather simple question. How difficult is it to produce $30 \mathrm{kPa}$ of pressure?

The simplest method to answer this question is to use everyday garments such as gloves and boots to see how much pressure they produce and how comfortable they are to the wearer. Finding out how difficult it is to achieve the desired $30 \mathrm{kPa}$ of pressure with these components will provide the means to produce an adequate baseline to compare active and elastic pressure production mechanisms as a way to quantifying performance.

In order to investigate this topic, some off-the-shelf compression stockings, as well as custom-made designs were ordered, but none created pressures larger than $100 \mathrm{~mm} \mathrm{Hg}$ (about $13.3 \mathrm{kPa}$ ). This pressure is less than is required for a MCP garment, but it is a significant fraction of the required pressure, suggesting that perhaps compression stockings or sleeves could be worn as comfort undergarments, reducing the total pressure needed to be produced by the actual MCP garment itself. Reducing the required MCP pressure simplifies the mechanical design and material properties requirements outlined in the previous section.

After examining other common garments such as ski boots which are capable of producing high pressures, a roller blade boot was selected to determine the pressure production capability of a common mechanical boot clasp. The particular type of roller blades in question have buckles, laces, and Velcro in order to create pressure. Figure 10 provides a good image of how the test was conducted. Note that the Velcro strap is not yet tightened, while the laces are tied, and the buckle is firmly in place. The TekScan sensor is used to record pressure, and will be discussed in much greater detail in later sections.

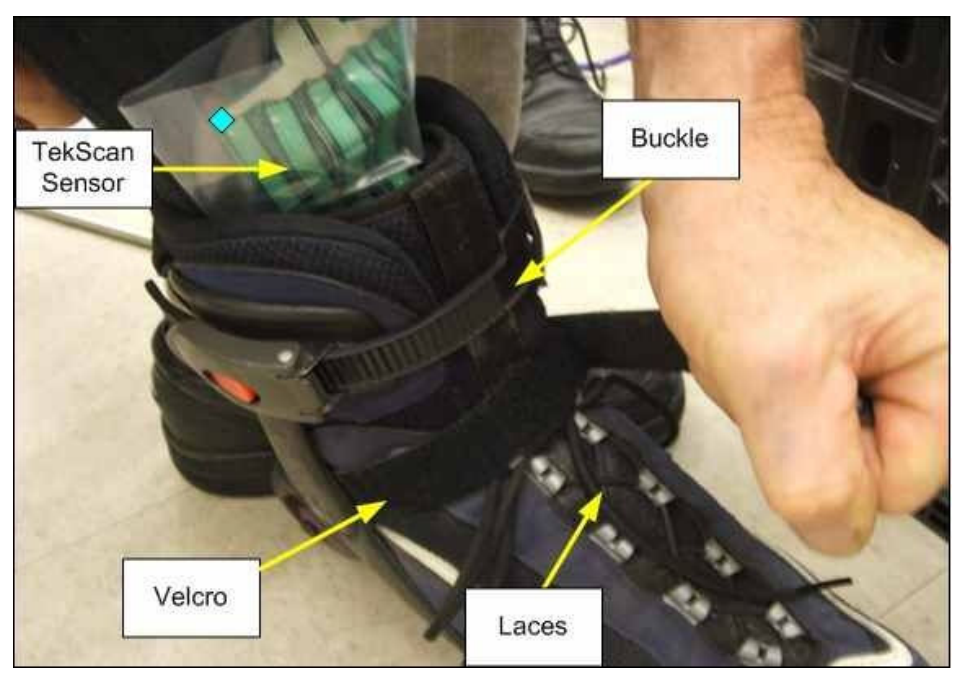

Figure 10: Roller blade testing

From this testing, it was determined that even with a simple, everyday device, it was straightforward to produce over the $30 \mathrm{kPa}$ of pressure required for the BioSuit. The following 
table illustrates the data recorded after the maximum pressure has been produced. The data above $28.4 \mathrm{kPa}$ (taking into account the $1.6 \mathrm{kPa}$ allowance of pressure differential) is highlighted. From the perspective of facing the leg straight on, the top-left corner of the sensor (represented by the diamond) is seen in the top-left corner of the data table. Reading down the columns provides values lower on the leg and onto the foot of the subject.

Table 7: Pressure readings on anterior of foot/lower leg $(\mathrm{kPa})$

\begin{tabular}{|c|c|c|c|c|c|}
\hline 0.00 & 0.00 & 0.00 & 0.00 & 0.00 & 11.33 \\
\hline 0.00 & 0.00 & 0.00 & 7.20 & 10.80 & 11.73 \\
\hline 18.13 & 19.47 & 0.00 & 0.00 & 0.00 & 0.00 \\
\hline 15.33 & 17.20 & 19.47 & 6.53 & 0.00 & 7.20 \\
\hline 15.73 & 20.40 & 29.33 & 13.47 & 24.00 & 27.60 \\
\hline 26.40 & 28.93 & 46.66 & 58.80 & 42.13 & 50.13 \\
\hline 45.20 & 50.40 & 62.53 & 41.46 & 44.26 & 62.53 \\
\hline 42.53 & 46.93 & 45.33 & 40.40 & 40.26 & 45.86 \\
\hline 17.87 & 20.40 & 17.47 & 19.47 & 22.00 & 13.87 \\
\hline 6.53 & 5.87 & 10.40 & 6.53 & 12.13 & 8.80 \\
\hline 5.07 & 8.80 & 18.80 & 15.07 & 17.47 & 35.06 \\
\hline 0.00 & 15.73 & 48.93 & 15.73 & 22.00 & 52.93 \\
\hline 6.53 & 18.53 & 23.33 & 27.60 & 29.06 & 35.73 \\
\hline 6.53 & 0.00 & 0.00 & 15.07 & 19.87 & 42.40 \\
\hline
\end{tabular}

From the table it can be seen that this pressure easily reaches $30 \mathrm{kPa}$. The subject commented that the pressure produced during this test did not feel like significantly more pressure than during normal usage.

The next question to answer is whether or not this pressure is being felt evenly around the crosssections of the leg. The next table displays the pressures on the posterior of the leg under the same conditions. Fewer rows are evident here, because simply due to leg geometry, less of the sensor could be placed inside the roller blades. Once again the pressures above $28.4 \mathrm{kPa}$ are highlighted. 
Table 8: Pressure readings on posterior of foot/lower leg $(\mathrm{kPa})$

\begin{tabular}{|c|c|c|c|c|c|}
\hline 0.00 & 0.00 & 0.00 & 0.00 & 6.53 & 12.13 \\
\hline 16.80 & 28.13 & 21.06 & 22.26 & 30.40 & 0.00 \\
\hline 30.53 & 29.86 & 27.33 & 22.53 & 30.53 & 0.00 \\
\hline 22.80 & 23.06 & 0.00 & 23.06 & 24.53 & 26.13 \\
\hline 18.53 & 24.53 & 23.33 & 24.53 & 25.33 & 0.00 \\
\hline 13.47 & 18.13 & 18.80 & 27.60 & 18.80 & 12.13 \\
\hline 13.07 & 15.73 & 27.86 & 19.87 & 17.20 & 0.00 \\
\hline 13.87 & 12.67 & 26.66 & 26.40 & 19.87 & 10.80 \\
\hline 5.87 & 13.87 & 22.80 & 8.80 & 0.00 & 0.00 \\
\hline 0.00 & 0.00 & 7.73 & 7.73 & 0.00 & 0.00 \\
\hline 5.07 & 6.53 & 0.00 & 0.00 & 0.00 & 0.00 \\
\hline
\end{tabular}

There are fewer places with the desired pressure in this table, but there are a large number of points within the $20 \mathrm{kPa}$ to $28 \mathrm{kPa}$ range. The shape of the roller blades, and the solid nature of the backing against the heel as opposed to the mesh in the front for tightening, may be one reason for this difference. Also, since the roller blades had to be loosened in order to move the sensor, the pressure on this recording may have been lower than that in Table 7.

To complete the measurements of the cross-section, both the interior and exterior pressure readings of the ankle were measured as well.

Table 9: Pressure readings on interior of the ankle $(\mathrm{kPa})$

\begin{tabular}{|c|c|c|c|c|c|}
\hline 5.87 & 7.73 & 11.33 & 6.53 & 5.07 & 0.00 \\
\hline 9.33 & 21.33 & 17.87 & 9.87 & 7.73 & 7.20 \\
\hline 15.73 & 46.80 & 46.13 & 16.53 & 12.67 & 4.27 \\
\hline 15.73 & 62.53 & 49.20 & 11.73 & 8.80 & 8.80 \\
\hline 7.73 & 12.13 & 25.60 & 8.27 & 12.13 & 5.87 \\
\hline 5.87 & 5.87 & 5.87 & 7.73 & 0.00 & 5.87 \\
\hline 7.73 & 22.53 & 26.13 & 18.80 & 8.80 & 0.00 \\
\hline 19.87 & 11.33 & 25.60 & 0.00 & 0.00 & 0.00 \\
\hline 18.80 & 19.47 & 7.73 & 0.00 & 0.00 & 7.73 \\
\hline 0.00 & 0.00 & 0.00 & 0.00 & 0.00 & 7.73 \\
\hline
\end{tabular}

The highlighted portions here show the location of the ankle, as they have the highest pressure. After the design has progressed beyond the calf, knee, and thigh to include the foot, this could potentially be a major issue. A similar result can be seen in Table 10. 
Table 10: Pressure readings on exterior of ankle $(\mathrm{kPa})$

\begin{tabular}{|c|c|c|c|c|c|}
\hline 15.07 & 9.33 & 9.33 & 14.27 & 10.80 & 0.00 \\
\hline 7.20 & 13.07 & 8.27 & 9.33 & 13.07 & 8.27 \\
\hline 7.20 & 11.73 & 6.53 & 0.00 & 20.40 & 27.33 \\
\hline 17.47 & 9.87 & 0.00 & 16.80 & 29.06 & 40.00 \\
\hline 7.20 & 13.47 & 5.87 & 32.00 & 39.60 & 62.53 \\
\hline 7.73 & 12.67 & 23.06 & 48.53 & 46.66 & 30.13 \\
\hline 5.07 & 5.87 & 16.13 & 16.80 & 22.80 & 6.53 \\
\hline 4.27 & 8.27 & 9.87 & 15.73 & 0.00 & 6.53 \\
\hline 5.87 & 7.73 & 0.00 & 18.53 & 0.00 & 11.73 \\
\hline 14.67 & 27.33 & 0.00 & 0.00 & 9.87 & 11.33 \\
\hline 8.80 & 17.87 & 0.00 & 10.80 & 0.00 & 10.80 \\
\hline
\end{tabular}

The extrusion of the ankle from the rest of the cross-section significantly increases the pressure at those points, leading to a large range of pressures over one cross-section. This data does not just represent the ankle however, and it lends credence to the assumptions that other extrusions, such as a bent knee, a bent elbow, or even the shin, would logically have higher pressures as well.

While this level of pressure is easily producible, careful notice needs to be taken of body geometry to ensure constant pressure around a circumference. 


\section{Chapter 3: Theoretical modeling of MCP in Matlab}

\subsection{Motivation}

In order for a spacesuit design based on the theory of mechanical counterpressure, such as the BioSuit, to become a viable alternative to the current gas-pressurized spacesuits, it must be shown that astronauts can function safely in a vacuum or near vacuum envionment for multiple EVA's, each of which can last up to 8 hours, over a period of several days. The longest test of a MCP system lasted slightly over one hour, which is much less than the duration of an average EVA. Therefore, the question of whether MCP-based spacesuit designs are indeed safe for human use is still left unanswered.

It is relatively simple to provide a large amount of pressure on the body with elastic wraps. Some compression stockings, such as those made by Jobst Inc., have been produced to provide up to 100 $\mathrm{mm} \mathrm{Hg}(\sim 13.3 \mathrm{kPa})$ of pressure on legs. Producing the pressure is possible with the proper material, as was discussed in the previous chapter, and up to $225 \mathrm{~mm} \mathrm{Hg}$ of MCP has been produced on the human body $[1,2,14]$. Therefore, the key unknown is not how much pressure can be produced on the human body via MCP, but how constant the pressure is over the body. The average pressure around a cross-section of a given part of the body can be adjusted by varying the force in the circumferential bands around that cross-section. (A constant average pressure can be created along the axial direction of a tapered cylinder by increasing the average circumferential force as the radius increases.) Instead, the focus must be on the magnitude of the pressure variation at different parts of the body.

To analyze different parts of the body, this thesis shall consistently refer to "cross-sections", which are defined as "slices" of a human body in a plane normal to the vertical axis of the human torso. In other words, if a human is standing upright on a flat surface, the "slices" are taken parallel to the floor. This figure, which is courtesy of the Visible Human Project, a program of the National Institutes of Health, displays a cross-section of the legs. Ideally, the thickness of a cross-section is infinitesimally small, but for the purposes of this thesis, a cross-section will defined as a "slice" of the body less than $5 \mathrm{~mm}$ thick, as the circumference of the body should not change significantly over that distance. 


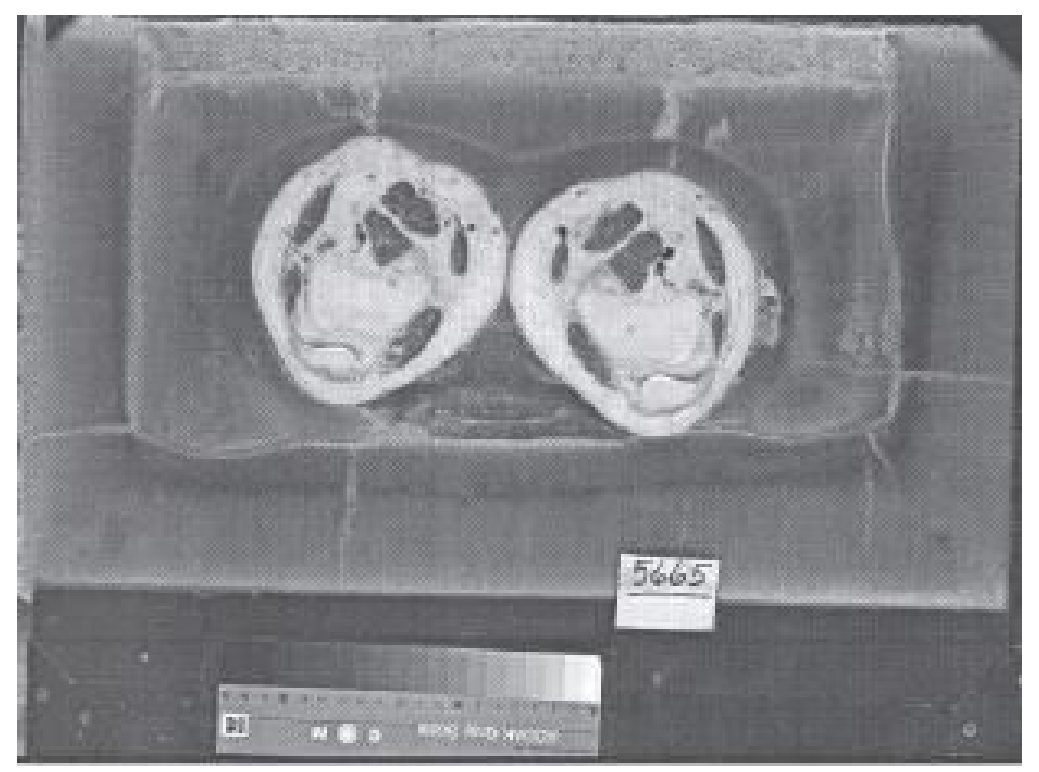

Figure 11: Visible Human Project thigh cross-section

However, the maximum variation of pressure around the circumference of a given cross-section that will not cause edema is unknown and requires further research. The design $\operatorname{target}^{7}$ for the variation of pressure is currently a conservative $1.6 \mathrm{kPa}$ as this pressure differential is known not to cause edema [16].

It is important to note that every cross-section of a human leg has a variable radius of curvature, since human legs are not perfect tapered cylinders. Therefore, the local radii of curvature can vary significantly from the average radius of curvature of any given cross-section on the human body. Due to this change in the radius of curvature, the local pressure on the body must vary as well, as described in Equation 2 shown in the previous chapter.

If the astronauts experience small amounts of edema, the effect may nto be harmful, as the swelling would merely even out the applied pressure on the skin. However, significant edema is not only uncomfortable, but can lead to more serious conditions, such as pre-syncope and syncope. Therefore, it is critical to understand how much pressure variation astronauts wearing a MCP-based spacesuit would experience, and the purpose of this chapter is to analyze this unknown using theoretical models.

\subsection{Methodology}

In order to model a MCP-based spacesuit design such as the BioSuit, the first equation outlined in Chapter 2 is used to relate pressure, tension, and body radius, which is derived from the law of thinwalled pressure vessels and the definition of stress.

\footnotetext{
${ }^{7}$ This value is probably overly conservative, as mentioned in the previous chapter.
} 
Before the design process can begin, however, other restrictions, in addition to the tolerances of the human body, must be noted. While we can postulate a skintight suit with any elastic properties we care to prescribe (e.g. specify elastic modulus, allow anisotropic properties), certain primary constraints must be met.

1. The skin-tight elastic garment is assumed to slide frictionlessly along the skin. Since the astronaut will almost certainly wear a comfort garment underneath the pressure layer, this constraint should not be hard to meet, since it involves the coefficient of friction of the elastic layer with the comfort garment, whose properties can be controlled, rather than with the human skin, whose properties are hard to control and are variable (sweating, etc.)

2. For a cylindrical surface, pressure is produced solely by circumferential force. Elastic fibers can be wrapped at various spiral angles around a cylindrical surface, but only the circumferential component of the stress in the fibers contributes to pressurizing the cylinder. Therefore, from a pressurization point of view, the elastic garment can be conceived of as a series of circumferential elastic bands joined by longitudinal fibers whose elastic modulus is not linked to the properties of the circumferential bands.

3. The average pressure on a cross-section can be adjusted by varying the force in the circumferential band. A constant average pressure can thus be created along the axial direction of a tapered cylinder by increasing the average circumferential force as the radius increases.

4. The tension and width are assumed to be constant around a cross-section. Given formfitting supporting structures on the side of the leg, similar to a splint, the material could be split into multiple parts, but this design would be far more complex and was not considered here.

With these assumptions, the development of the model can begin. In order to model MCP, the first step is to solve for the tension, using a desired pressure (in this case, $30 \mathrm{kPa}$ ), and the average crosssectional body radius. Two different data sets were made available to the author, each of which consists of points on the human skin in Cartesian (x,y,z) coordinates in order to create the model. Cross-sections were defined for each data set, and then the average radius of curvature is easily computed by measuring the circumference around the leg and simply dividing by $2 \pi$. In the model, the circumference is determined by adding the straight-line distances between each of the points in a cross-section. The relative spacing between the points introduces some amount of error into the equation already, especially for the regions with a larger radius (ie the thigh).

Given the desired pressure to be produced and the average cross-sectional body radius, then the overall tension on a cross-section is easily computed. Around each circumferential cross-section, the tension must be constant. If the body part is irregular, with non-circular cross-sections, then the local pressure around a cross-section of an irregular cylinder will vary inversely with the local radius of curvature. Varying the average force in the circumferential band cannot change this pressure variation, caused by the changing radius of curvature along a non-circular curve. This variation is present regardless of what kind of material is used, i.e. there is no difference in this respect between passive and active elastic materials. 
To calculate the local pressure, the radius of curvature at each point in the cross-section must be defined. Two methods were used for finding this radius, each of which is outlined below.

\subsubsection{The Neighbor Normal Method}

The local radius of curvature in the Neighbor Normal Method is found using a simple equation:

$$
\mathrm{r}=\mathrm{ds} / \mathrm{d} \theta
$$

where

$\mathrm{ds}=$ straight line distance from point to point, $\mathrm{mm}$

$\mathrm{d} \theta=$ difference in angle from point to point, radians

The length ds is easily found using the straight line distances between neighboring points. The procedure for solving for $\mathrm{d} \theta$ is a bit more complicated, and borrows significantly from the work of Wolfrum. In her thesis, the normals at each individual point were calculated by taking the vector from the point of interest to each of its surrounding neighbors. Then the cross products of the vectors were taken (following the right-hand rule) to create a vector normal to those vectors, as displayed by the vectors $\mathrm{n} 1$ through $\mathrm{n} 8$ in Figure 12 . These vectors were then averaged, to determine a vector at that point perpendicular to the leg, labeled ntotal [12]. The component of this vector within the plane of the cross-section is then found by simply removing the component of the vector perpendicular to the cross-section. The dot product of this two-dimensional vector, labeled as $\mathrm{n}_{\text {total }}$ in Figure 12, along with the two-dimensional vector of the neighboring points is used to determine the value of $\mathrm{d} \theta$.

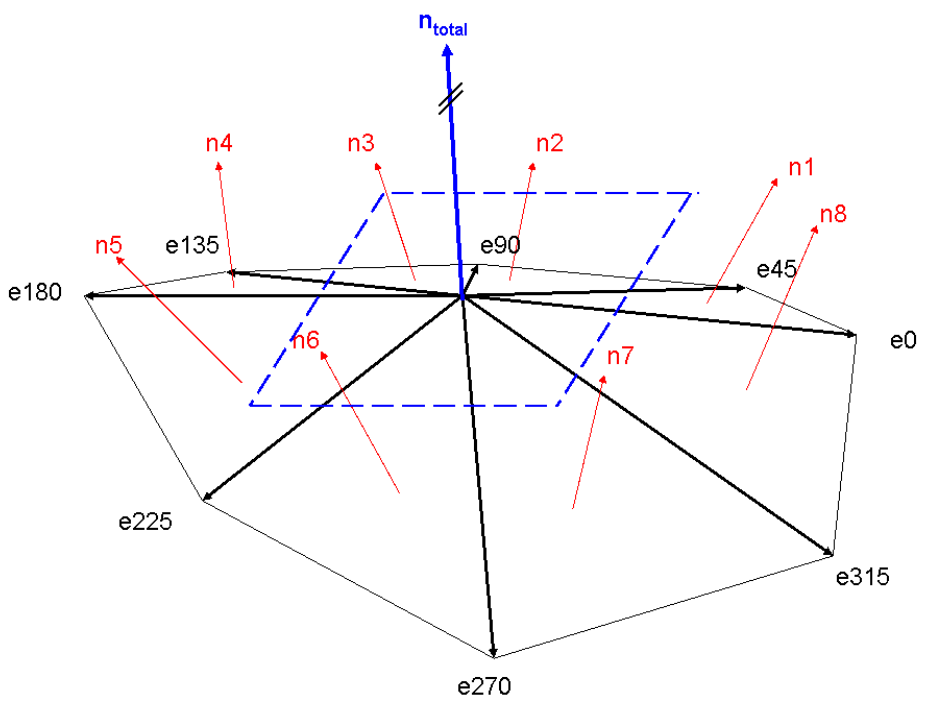

Figure 12: Visual explanation for determining Neighbor Normal Method vectors ${ }^{8}$

\footnotetext{
${ }^{8}$ Figure courtesy of Nina Wolfrum
} 
The points e0, e45, e90, e135, e180, e225, e270 and e 315 are the neighbors to the central point. Each of the small normal vectors is the local normal vector, while $n_{\text {total }}$ is the actual normal vector at this central point. The value $\mathrm{d} \theta$ is calculated by taking dot products of the $\mathrm{n}_{\text {total }}$ vectors from neighboring points.

Having solved for ds and $\mathrm{d} \theta$, the local radius of curvature, and therefore, the local pressure, is easily discovered. See Appendix E for the computer code.

\subsubsection{Point Circular Method}

This method uses the fact that 3 points define a circle. The coordinates of the desired point and 2 of its neighbors are input into the code, and then the perpendicular bisectors between the points are found. The intersection of these lines is the center of the newly defined circle, as the perpendicular bisector of any chord on a circle extends through the center of that circle, which can be seen in Figure 13.

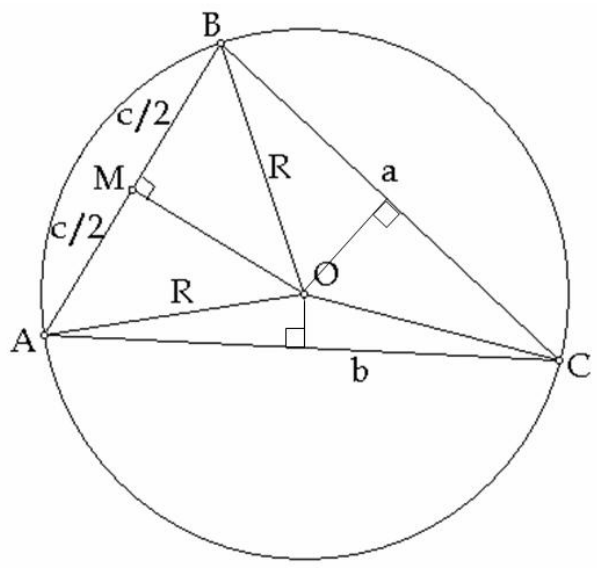

Figure 13: Method for determining the center of curvature in the 3 Point Circular Method ${ }^{9}$

In this analysis the point of interest is the "center" point, since it is located in the center of the 3 points, and defines the location of the radius of the curvature. The other 2 points are symmetrically located around the center point, based not on distance, but rather on the number of points in between them and the center point. There was some concern that using points directly next to each other would lead to inaccurate local radii of curvature, as even relatively small errors inherent to the scan data could cause large changes in the radii of curvature that are not representative of the actual human body. Taking points farther from each other would allow the errors to cancel each other out. Figure 14 displays visually what the previous text explains in words. If the method is to skip 3 points, the center point (called $\mathrm{C}$ ) and the 2 points in circles would be used to find the local radius. If 7 points are skipped, then the center point and the points in the boxes would be used instead. See Appendices $\mathrm{E}$ and $\mathrm{F}$ for the computer code.

\footnotetext{
${ }^{9}$ Figure courtesy of Graeme McRae: http://mcraeclan.com/MathHelp/GeometryTriangleCenterCircumscribedCircle.htm
} 


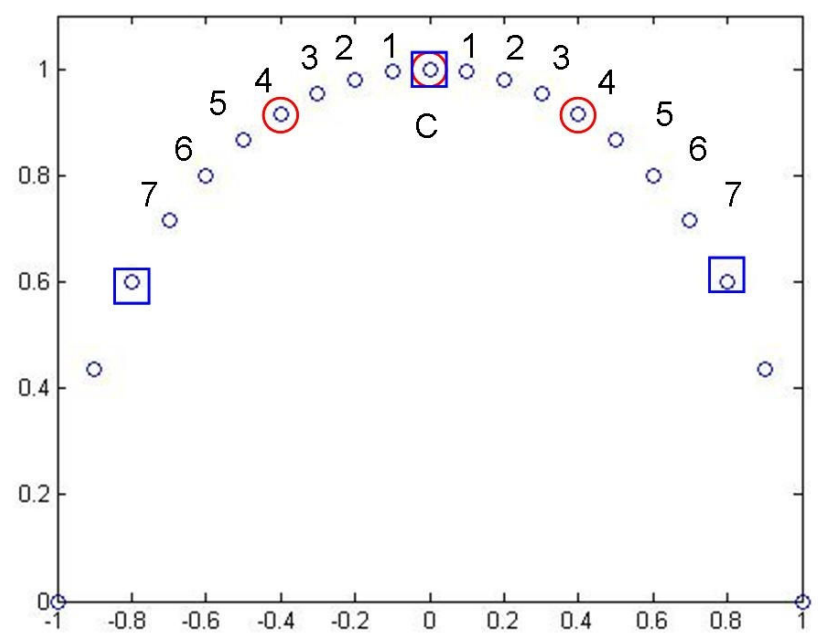

Figure 14: Explanation of point skipping

While the methods differ in the determination of the local radius of curvature, they should provide approximately the same answer, and in doing so, provide a level of verification that the theoretical values obtained from this analysis are accurate.

\subsection{Analysis using Wolfrum data}

In Wolfrum's thesis, 6 subjects had their right legs scanned in a determination of skin strain. In this chapter, these scans are utilized for the purpose of determining the pressure variation over crosssections of the human leg. Each subject had 9 columns of points, with 10-12 rows per subject. The two methods described in the previous section were all used to determine the pressure distribution [12].

\subsubsection{The Neighbor Normal Method}

Due to the use of the neighboring points to define the local radius of curvature, both the top and bottom data rows are unable to be used in this analysis. With this limitation understood, the results of the calculated pressure distributions for each of the 6 subjects in the Wolfrum thesis using the Neighbor Normal method are shown in the following figures. The color bar displays the modeled pressures on the skin in $\mathrm{kPa}$, and the leg dimensions (x,y,z coordinate system) are in $\mathrm{mm}$. The goal is to maintain pressure at $30 \mathrm{kPa}$, as described previously. 

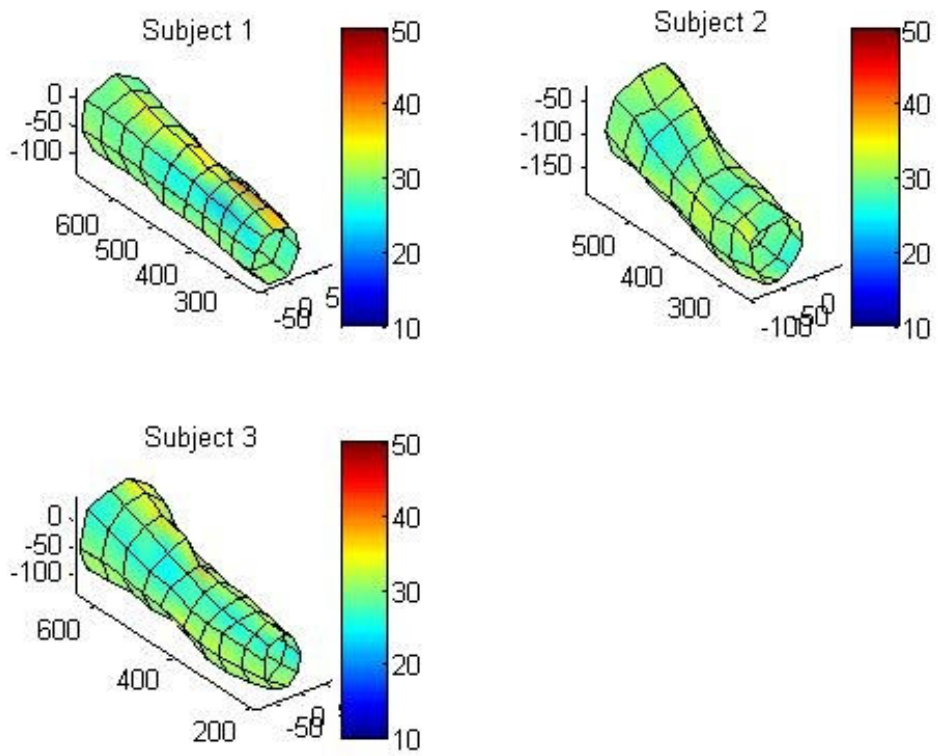

Figure 15: Modeled pressures for Subjects 1-3 in the Wolfrum thesis
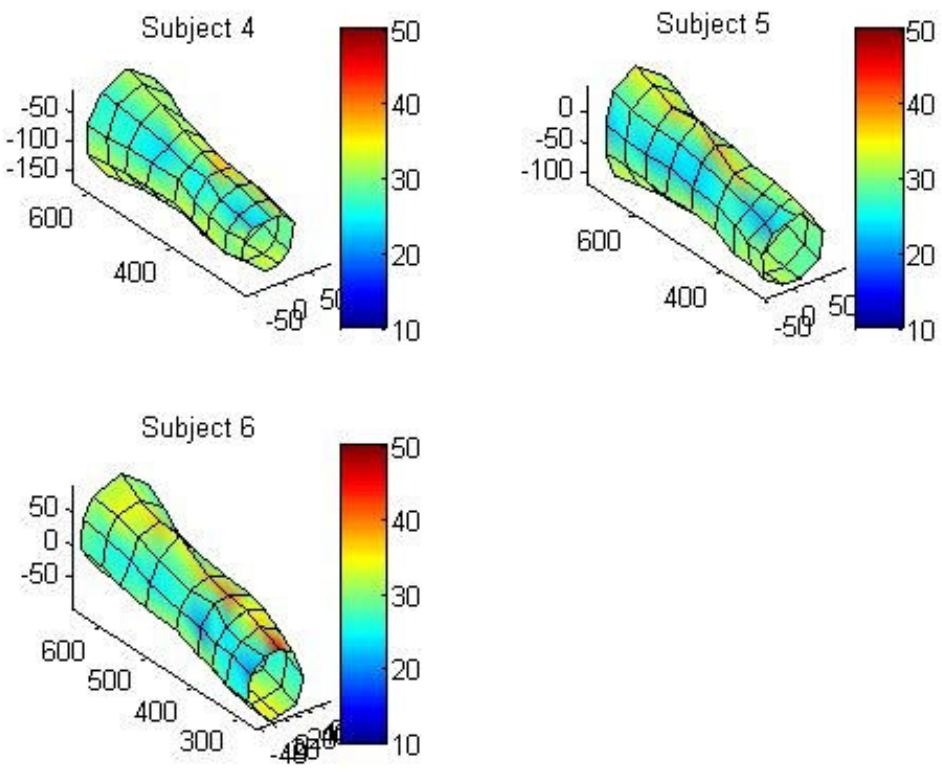

Figure 16: Modeled pressures for Subjects 4-6 in the Wolfrum thesis

While these pictures are useful for giving a brief overview of the ranges, they do not provide a comprehensive look at the pressures. It is fairly obvious the range of pressure distribution is between 20 to $40 \mathrm{kPa}$ for most of the subjects, and the highest pressure is on the anterior of the leg, most especially the knee and the anterior of the shin, which is logical since those areas of the body 
have the smallest local radius of curvature. However, a more detailed analysis is useful for understanding why the model produces these pressures. To help explain the results, more background on the data organization must be given.

Each cross-section (row) has 9 points, which are counted with point 1 along the anterior of the shin, and each subsequent column is clockwise around the leg from a top-down perspective, as seen in Figure 17.

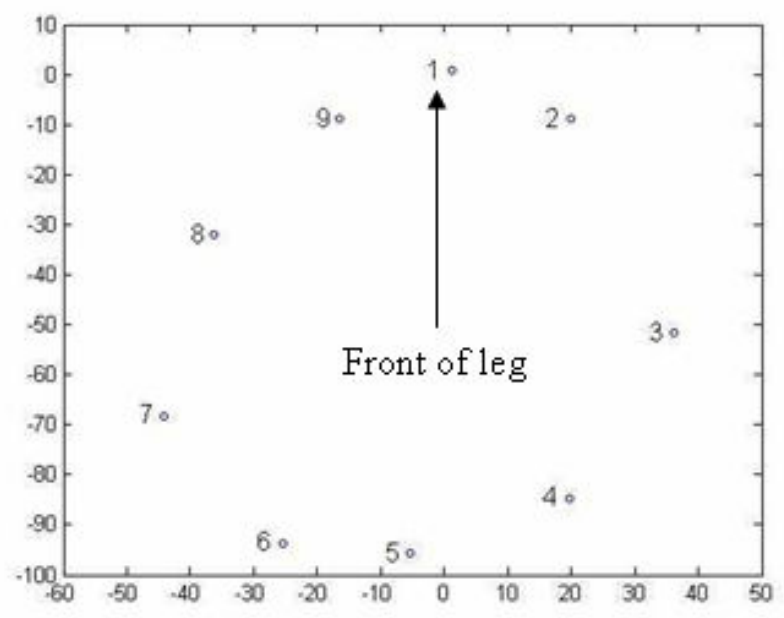

Figure 17: Explanation of numbering system for every cross-section (top view)

The following table outlines the data for Subject 5 in the Wolfrum study, with the columns numbered as just described. Row 1 is the cross-section of the lower shin, and the number of each row represents a cross-section higher on the leg, until the upper thigh is reached (Row 9). Each pressure in the table is in $\mathrm{kPa}$. Note that each row should have an average pressure of $30 \mathrm{kPa}$, although due to the removal of a portion of the normal vectors, there is some rounding error. 
Table 11: Point by point pressure for Subject 5

\begin{tabular}{|c|c|c|c|c|c|c|c|c|c|c|c|}
\hline \multicolumn{12}{|c|}{ Column } \\
\hline \multicolumn{6}{|c|}{ Front of the leg (clockwise from top) } & \multicolumn{6}{|c|}{ posterior of the leg } \\
\hline \multirow{3}{*}{$\begin{array}{l}\text { Lower } \\
\text { shin }\end{array}$} & & 1 & 2 & 3 & 4 & 5 & 6 & 7 & 8 & 9 & Avg \\
\hline & 1 & 26.34 & 22.94 & 31.01 & 31.46 & 32.60 & 28.80 & 27.91 & 29.08 & 36.10 & 29.58 \\
\hline & 2 & 26.13 & 21.32 & 28.53 & 33.21 & 32.80 & 28.92 & 28.61 & 29.91 & 33.50 & 29.21 \\
\hline \multirow{4}{*}{ Row } & 3 & 28.24 & 23.00 & 27.46 & 28.93 & 34.94 & 30.50 & 28.24 & 30.72 & 33.08 & 29.46 \\
\hline & 4 & 36.64 & 27.11 & 25.37 & 31.94 & 35.20 & 28.73 & 27.68 & 28.83 & 29.52 & 30.11 \\
\hline & 5 & 37.72 & 23.60 & 22.90 & 32.38 & 32.83 & 30.06 & 29.62 & 26.72 & 29.66 & 29.50 \\
\hline & 6 & 35.59 & 24.91 & 22.80 & 30.25 & 33.37 & 31.13 & 32.80 & 25.67 & 26.09 & 29.18 \\
\hline \multirow{3}{*}{$\begin{array}{l}\text { Upper } \\
\text { thigh }\end{array}$} & 7 & 36.87 & 26.84 & 23.03 & 30.01 & 33.08 & 30.91 & 28.53 & 25.40 & 28.76 & 29.27 \\
\hline & 8 & 34.31 & 27.08 & 24.47 & 29.18 & 32.97 & 31.80 & 27.25 & 25.21 & 30.48 & 29.19 \\
\hline & 9 & 35.09 & 26.65 & 22.46 & 29.51 & 33.70 & 31.57 & 27.86 & 25.13 & 29.97 & 29.10 \\
\hline
\end{tabular}

Immediately obvious is the wide variation of values from a minimum of $21.32 \mathrm{kPa}$ to a maximum of $37.72 \mathrm{kPa}$, which is a range of $\pm 8.2 \mathrm{kPa}$. It should be further noted here that this subject did not have the largest difference between the smallest and largest pressure values. Subject 6 had a theoretical pressure at the front of the shin of $44.5 \mathrm{kPa}$ (the only subject with a pressure over 40 $\mathrm{kPa}$ ) and a minimum of $20.59 \mathrm{kPa}$. The $\pm 10 \mathrm{kPa}$ variability exceeds the $\pm 1.6 \mathrm{kPa}$ which is the largest variability for which data exists to show physiological acceptability. The implications will be discussed later in this chapter.

\subsubsection{Point Circular Method}

Due to the small number of cross-section data points (only 9), no point skipping was used in this analysis. Only the center point and its 2 nearest neighbors were analyzed. Once again, a color overview of the results from this method, followed by a table of actual values and a representative cross-section will be given. First, the pictorial overview, as seen in the following two figures: 

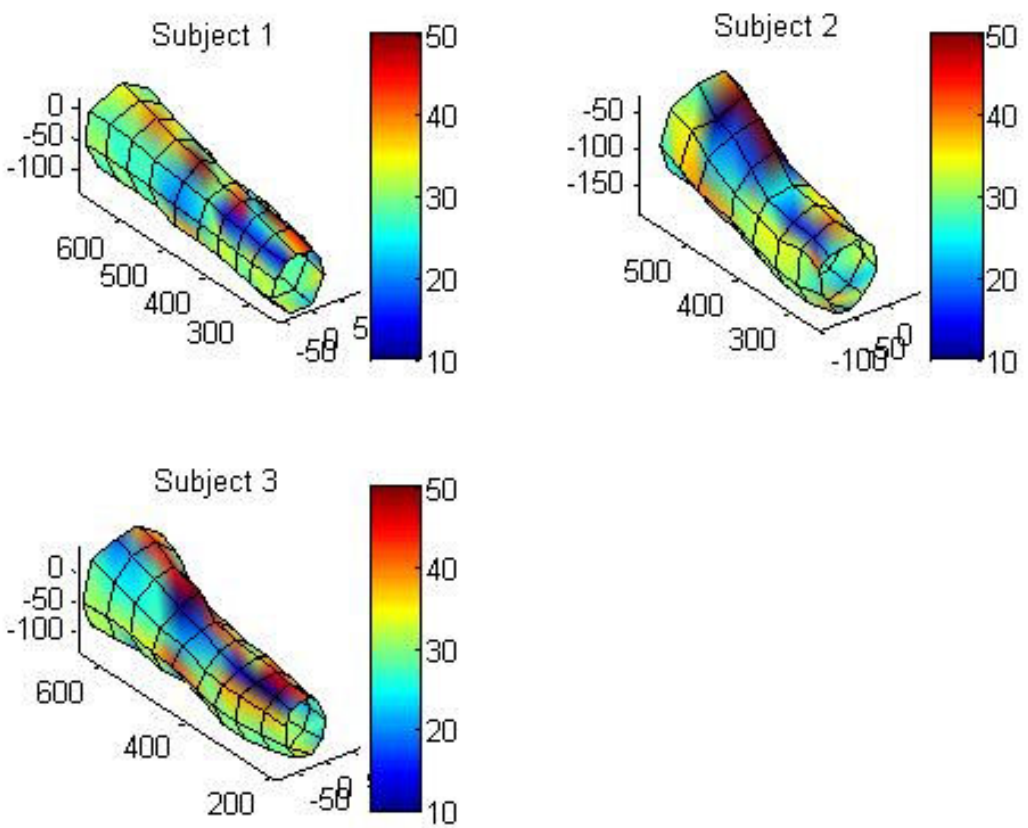

Figure 18: Modeled pressures for Subjects 1-3 in the Wolfrum thesis
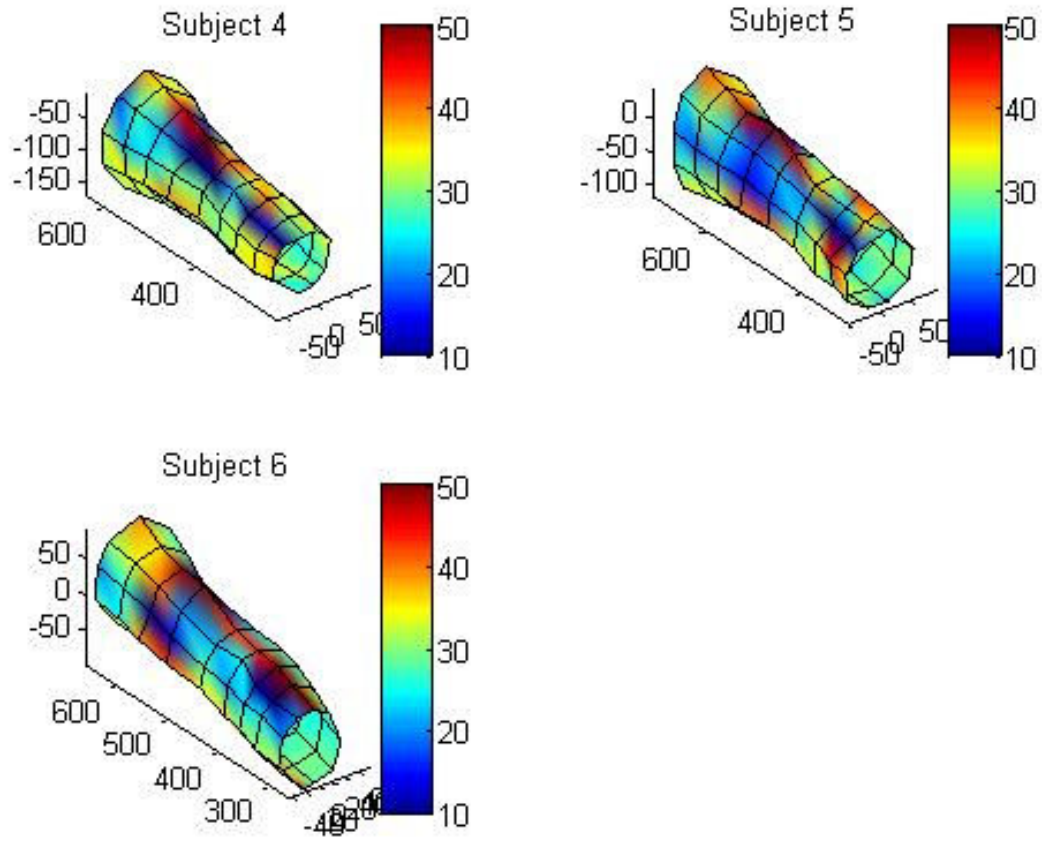

Figure 19: Modeled pressures for Subjects 4-6 in the Wolfrum thesis 
From these figures, it is obvious that the range of pressures is significantly higher using this method, and the highest pressures are around the kneecap and the anterior of the shin again, where they are expected to be for the theoretical models. The reason for this expectation is the relatively small radius of curvature at these points, as mentioned previously.

Once again, a detailed table for Wolfrum Subject 5 is useful for analyzing the calculated pressures. As in the previous example, the first column is the very front of the shin, with the rest of the columns proceeding in a clockwise manner, from an overhead perspective. The average pressure along each horizontal row should average $30 \mathrm{kPa}$, although using this method involves some error, since the circumferential measurements determine the average pressure, but the local pressure is determined based on the local radius of curvature, which is defined by a different methodology.

Table 12: Point by point pressure for Wolfrum Subject $5(\mathrm{kPa})$

\begin{tabular}{|c|c|c|c|c|c|c|c|c|c|c|c|}
\hline \multicolumn{12}{|c|}{ Column } \\
\hline \multicolumn{6}{|c|}{ Front of the leg (clockwise from top) } & \multicolumn{6}{|c|}{ posterior of the leg } \\
\hline \multirow{2}{*}{$\begin{array}{c}\text { Lower } \\
\text { shin }\end{array}$} & & 1 & 2 & 3 & 4 & 5 & 6 & 7 & 8 & 9 & Avg \\
\hline & 1 & 26.52 & 16.37 & 35.04 & 36.01 & 30.37 & 22.93 & 28.33 & 30.24 & 39.24 & 29.45 \\
\hline \multirow{5}{*}{ Row } & 2 & 36.09 & 2.71 & 44.84 & 26.45 & 32.13 & 28.52 & 30.51 & 24.81 & 39.62 & 29.52 \\
\hline & 3 & 23.41 & 7.62 & 33.26 & 34.27 & 32.99 & 24.00 & 29.51 & 24.91 & 43.19 & 28.13 \\
\hline & 4 & 36.89 & 20.23 & 22.64 & 34.03 & 34.56 & 28.34 & 23.10 & 31.27 & 32.38 & 29.27 \\
\hline & 5 & 45.99 & 21.37 & 16.97 & 40.97 & 28.17 & 27.62 & 33.57 & 23.98 & 28.12 & 29.64 \\
\hline & 6 & 55.91 & 17.42 & 14.62 & 39.18 & 31.25 & 28.71 & 36.02 & 22.91 & 14.15 & 28.91 \\
\hline \multirow{3}{*}{$\begin{array}{l}\text { Upper } \\
\text { thigh }\end{array}$} & 7 & 47.25 & 23.03 & 19.99 & 32.58 & 32.29 & 33.92 & 26.83 & 26.41 & 22.49 & 29.42 \\
\hline & 8 & 38.14 & 29.86 & 18.04 & 28.37 & 34.52 & 34.11 & 30.30 & 13.94 & 35.16 & 29.16 \\
\hline & 9 & 41.28 & 23.85 & 20.03 & 26.17 & 36.25 & 34.37 & 24.75 & 20.04 & 33.10 & 28.87 \\
\hline
\end{tabular}

The extreme outliers, defined as pressures above $50 \mathrm{kPa}$ or below $10 \mathrm{kPa}$, are highlighted in green and yellow respectively. These values are the drivers for the large ranges.

\subsubsection{Verification}

An analysis was conducted on the data taken by Zhe Liang Sim in his thesis, to determine if there could be some experimental verification of the methods described previously. One subject (Subject 5 in the Wolfrum study) was in common between both studies, so that individual's data will be focused on here. Note that the data shown below (Table 13) is not calculated, rather it was measured by TekScan pressure sensors.

Overall, 6 "snapshots" were taken of this subject, with the timeline and pressures both in $\mathrm{mm} \mathrm{Hg}$ and $\mathrm{kPa}$ expressed below. These pressure values are an average pressure from the anterior of the calf of the subject. 
Table 13: Pressure on Wolfrum Subject 5 in Sim experiment [14]

\begin{tabular}{|c|c|c|}
\hline Time & Average & Average \\
\hline$(\mathrm{min})$ & $(\mathrm{mmHg})$ & $(\mathrm{kPa})$ \\
\hline 3 & 132 & 17.5 \\
\hline 22 & 154 & 20.5 \\
\hline 30 & 169 & 22.6 \\
\hline 42 & 163 & 21.7 \\
\hline 53 & 178 & 23.7 \\
\hline 67 & 154 & 20.6 \\
\hline
\end{tabular}

In this study, the pressure goal was $30 \mathrm{kPa}$, just as was set in the models, but it can be seen that the average is significantly below this level. Sim argued that because the shin tends to be flat or concave, the pressure actually is reduced in the region, and he noted that other scientists and engineers pursuing MCP suits have encountered the same problem over similar features on the body. His theory was that the bindings were actually producing a higher pressure on convex sections of the leg (such as the calf) because the subjects did not experience significant discomfort during testing [14]. However, this hypothesis is untested. The following table represents one snapshot (one reading from a given time period in the table above) for Subject 5 compared to the relevant points from the two methods discussed in great detail previously. Table 14 splits the data from Table 13 into 12 even sections, to correspond with 12 data points from both the Neighbor Normal and 3 Point Circular Method. All values are in $\mathrm{kPa}$, with the extreme outliers (more than 20 $\mathrm{kPa}$ from the goal of $30 \mathrm{kPa}$ ) highlighted.

Table 14: Comparison of computations to experimental data

\begin{tabular}{|c|c|c|c|c|c|c|c|c|}
\hline \multicolumn{3}{|c|}{ Sim data } & \multicolumn{3}{c|}{ Neighbor Normal Method } & \multicolumn{3}{c|}{ 3 Point Circular Method } \\
\hline & Center & & & Center & & & Center & \\
\hline 24.36 & 20.17 & 11.92 & 36.10 & 26.34 & 22.94 & 39.24 & 26.52 & 16.37 \\
\hline 25.42 & 22.96 & 20.30 & 33.50 & 26.13 & 21.32 & 39.62 & 36.09 & 2.71 \\
\hline 27.94 & 23.75 & 23.46 & 33.08 & 28.24 & 23.00 & 43.19 & 23.41 & 7.62 \\
\hline 25.79 & 18.96 & 6.00 & 29.52 & 36.64 & 27.11 & 32.38 & 36.89 & 20.23 \\
\hline
\end{tabular}

Since the values recorded by Sim used a TekScan sensor, which has a pressure measurement reading portion of $8 \mathrm{~cm}$ by $20 \mathrm{~cm}$, only a few of the data points from the Wolfrum scan could be compared, as the rest of these date points were located in portions in body not covered by the TekScan sensor. Therefore, some assumptions had to be made in order to make this comparison. It was decided that the 96 sensels of the TekScan sensor roughly corresponded to 3 columns and 4 rows of the Wolfrum data points, so the data from Sim was averaged accordingly. 
Obviously both methods predict significantly different pressures than those actually found, for reasons that will be discussed in the next section.

\subsubsection{Discussion}

The data from the Wolfrum thesis contains 2 major limitations, the first of which is the small number of data points, and the effects of this low data granularity on the two methods are quite significant.

For the Neighbor Normal Method, one good check is to look at the normal vectors being computed relative to the actual points on the body. The following figure is a representative sample of the computed normal vectors.

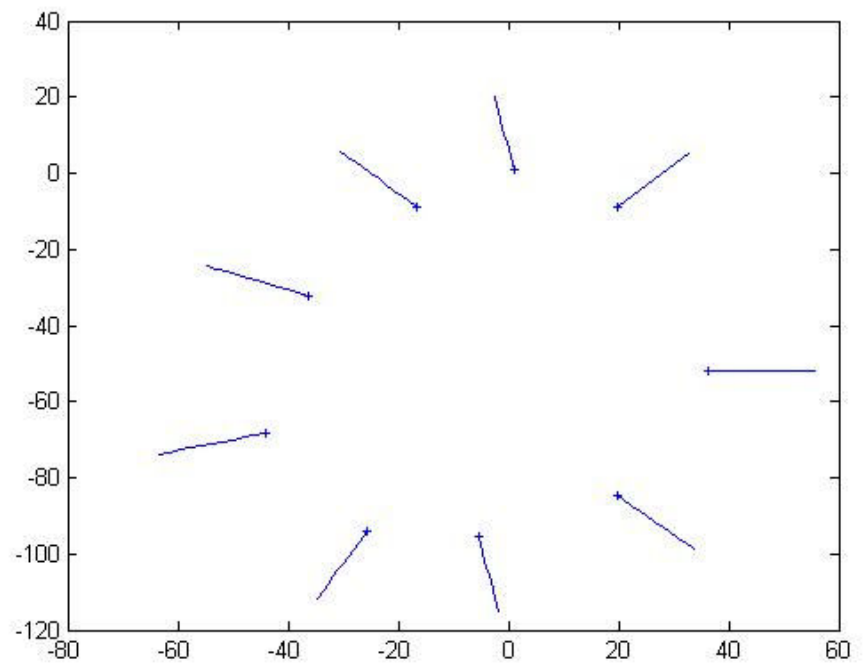

Figure 20: Smallest cross-section on Wolfrum Subject 6

In Figure 20, the front of the leg is at the $(0,0)$ point, just as in previous figures. Each ' + ' represents a scanned point on the body, while the straight lines are the normal vectors. Given the point as the very front of the leg, the expectation would be for the normal to be a vertical line, but this is obviously not the case. The point at roughly $(37,-50)$ however does have the perfectly horizontal normal expected of roughly the right edge of the leg. Once again, more points that are closer together would be useful in determining which method is most appropriate. More data points would likely reduce the error caused by the need to use straight lines and produce more accurate normal vectors.

For the 3 Point Circular Method an example cross-section, the fourth row from the top of the leg, from Wolfrum Subject 6 will be analyzed because it contains both a high $(56.73 \mathrm{kPa})$ and low $(3.84$ $\mathrm{kPa}$ ) outlier. The circles represent the points on the leg, while the stars represent the centers of the circles determined by each point and its two neighbors. 


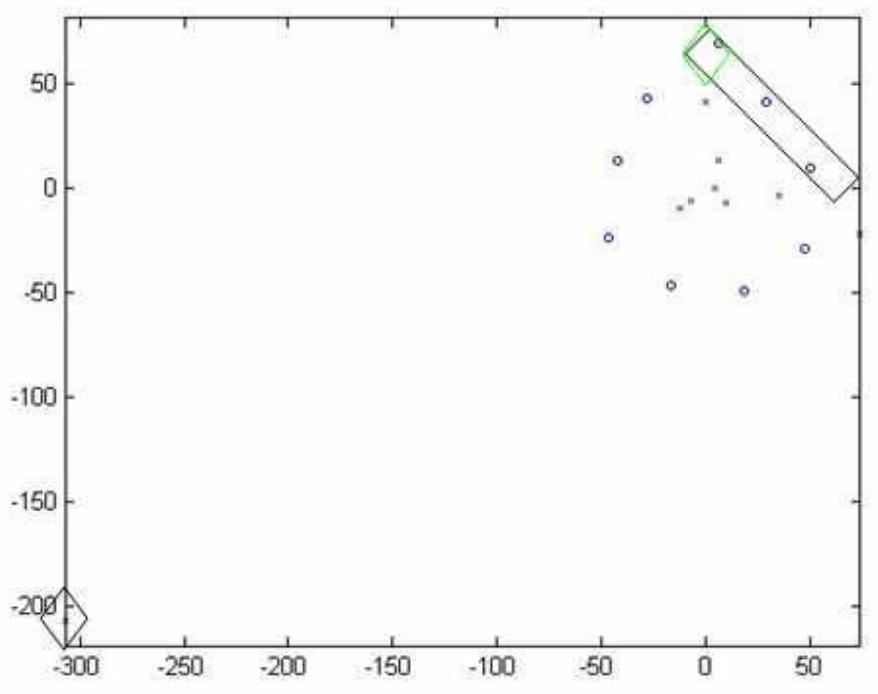

Figure 21: Cross-section on Wolfrum Subject 6

Note that there is 1 very large outlier: a point at the lower left of the figure, approximately (-300,215), centered in the black diamond. This is the center of the circle using the three points in the black rectangle at the upper right. The very large radius drives the pressure to very low levels, as seen in Equation 1. The three points are approximately in a straight line, which accounts for the very large radius. Obviously, straight lines do not model the leg properly (although the leg is flatter in this section), but given the fidelity and granularity of the data available, this is the result.

The very high pressure is based on the point in the center of the diamond in the top left corner of the rectangle, which is the front of the shin. The closeness of the center of the circle, which is probably somewhat inaccurate given the distance between the points on either side, drives the pressure to a very large level.

Having shown that the pressure distribution is affected by the granularity of the data, the second limitation must be discussed as well. It is important to note that all the data points were recorded with subjects under normal atmospheric pressure. When a subject is wrapped with MCP, the body "circularizes" or "deforms" in order to equalize the pressure production. Therefore, this data set does not accurately depict the true distribution of pressure when using MCP. However, it does provide a solid starting point for analyzing the pressure variation that is produced by the BioSuit or any spacesuit design based upon the principles of mechanical counterpressure.

Given the stated assumptions, the model outputs show considerable variability, and must be analyzed accordingly. These outputs should not be taken as actual predictors of the pressure variations a MCP suit would place on an individual, but rather as at least an upper bound on the values. For example, as detailed in Table 11, the pressure for that subject varies from $21 \mathrm{kPa}$ to 37 $\mathrm{kPa}$ in the model. This does not meet the design target (although the $1.6 \mathrm{kPa}$ range may be too conservative, as stated previously). However, knowing the inherent errors caused by the limited 
data, the results indicate that MCP could be a reasonable design. These results show the need for physiological testing to determine the acceptable limits of circumferential pressure variability.

\subsection{Analysis using Cyberware Inc. data}

Additional research led to the discovery of new human models with denser data that can alleviate the data granularity limitation, although no solution to the lack of data with human subjects experiencing MCP was discovered. Creating a custom data set was considered, but discarded due to the degree of difficulty of undertaking such an endeavor.

The four data sets used in this modeling analysis were obtained free of charge from Cyberware Inc. (http://www.cyberware.com/products/scanners/index.html), a company that creates scanning equipment. These scans were of the entire human body, so a few steps were undertaken in order to process the data. As some of the assumptions in these procedures affect the results, they will be outlined here.

1. For each individual, all the data points were removed from the file except those on the right leg between the ankle and the hip. ${ }^{10}$

2. The data was organized into cross-sections $4 \mathrm{~mm}$ thick. This value was used to obtain a reasonable number of data points per cross-section, while at the same time ensuring that the points were taken from approximately the same cross-section.

3. The cross-sections were spaced $5 \mathrm{~cm}$ apart in order to provide a significant number of crosssections without creating an unnecessarily long calculation time.

4. The data points were sorted into order around each of the circumferences of the crosssections. Data points too close to each other (within $1 \mathrm{~mm}$ in the $\mathrm{x}$ or $\mathrm{y}$ directions, the axes in the plane of the cross-section) were deleted, as they provided no additional value. Oftentimes, these "duplicate" points were located at the same radian value, but at a different radius, which would have created large errors in the calculations.

5. The number of data points was limited to a user-input value for each subject. The value was chosen to be the same as the number of points available for the smallest cross-sections, but still at least half of the largest cross-sections. The sampling was taken by using all the evennumbered points in the series around the circumference, and then adding the odd-numbered points with the largest total gaps from its neighbors, in order to find the points that best described the cross-section. The main reason for this step was to create a plot of the entire leg which displays the pressures based on a color scale. The pressures were interpolated between neighboring points, and if the number of points were not constant between the cross-sections, then the interpolation would not have worked properly.

\footnotetext{
${ }^{10}$ The decision to use the right leg was arbitrary, and done only to reduce computing time (the left leg should give similar results) as well as to match the experimental procedures outlined both in this thesis and the Sim thesis.
} 
With these data points organized, the pressure calculations began. Whereas in the previous section, 2 potential methods were discussed, only the 3-point circular method was adopted here, and the code is available in Appendix F. The Neighbor Normal Method would be difficult to use given the random distribution of the data points in the chosen cross-sections, as the points were not placed in vertical lines as in the previous study, but in a random fashion around the cross-section. Attempts to take cross products would have introduced large errors given the varying straight-line distances to be calculated. However, the code from the previous analysis could be modified without too much difficulty to create the normal vectors.

Following the method outlined above, an analysis was conducted on each of the 4 data files available. The following example figures are identical cross-sections on Cyberware Subject 1 . Figure 22 refers to a cross-section in which 3 points are skipped during the analysis to create the local radii. The centers for each of the data points are shown by the small squares. Obviously, some of these centers are significant distances from the surface of the leg, causing a large amount of variation in local radii. In contrast, Figure 23 displays the calculations when 7 data points are skipped. In this figure, the large variations have disappeared completely, and all the local centers of curvature are inside the leg.
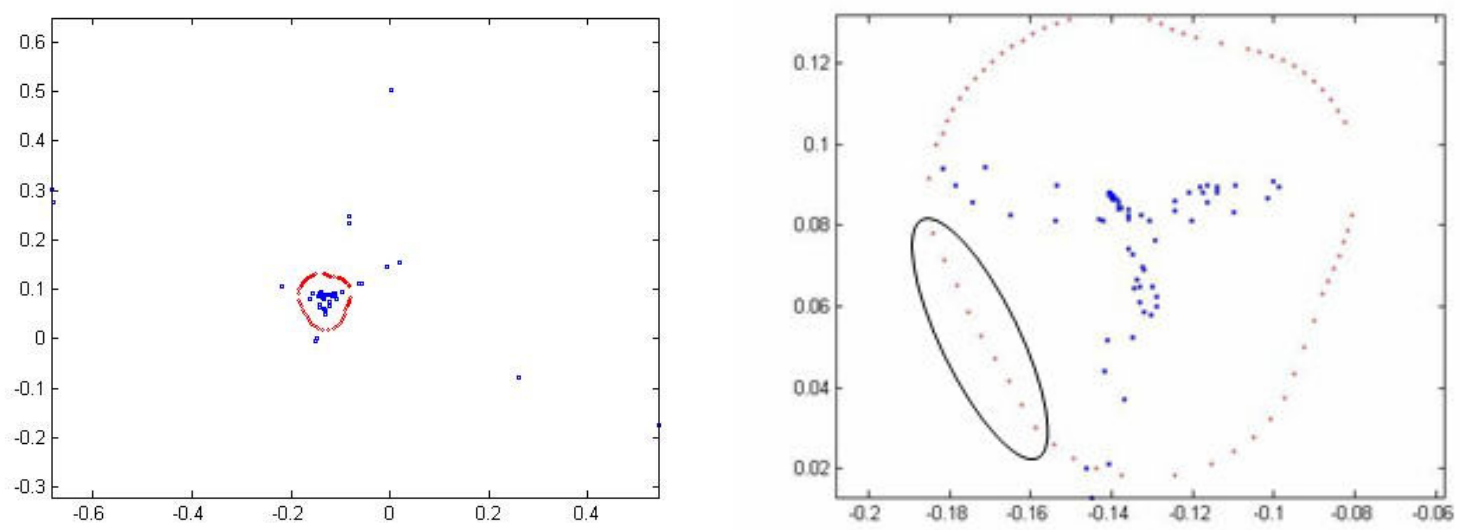

Figure 22: (left) Cross-section skipping 3 points

Figure 23: (right) Cross-section skipping 7 points

\subsubsection{Results}

As discussed above, varying the points used for determining the local radius affects the pressure values recorded. As a larger number of points are skipped, the value for each local radius draws closer to the average value for the entire cross-section. The following chart compares the number of points skipped for radii determination to the number of points that had local pressures within $5 \mathrm{kPa}$ of the desired $30 \mathrm{kPa}$ pressure. The $5 \mathrm{kPa}$ value was chosen for reasons that will be discussed shortly. 
Table 15: Table of Pressure percentages

\begin{tabular}{|c|c|c|c|c|}
\hline $\begin{array}{c}\text { Number of points } \\
\text { skipped }\end{array}$ & \multicolumn{4}{|c|}{ Percentage of points within 5 kPa of desired 30 kPa } \\
\hline & Subject 1 & Subject 2 & Subject 3 & Subject 4 \\
\hline 1 & $30 \%$ & $31 \%$ & $28 \%$ & $29 \%$ \\
\hline 2 & $36 \%$ & $39 \%$ & $37 \%$ & $36 \%$ \\
\hline 3 & $42 \%$ & $43 \%$ & $42 \%$ & $38 \%$ \\
\hline 4 & $45 \%$ & $48 \%$ & $46 \%$ & $44 \%$ \\
\hline 5 & $52 \%$ & $52 \%$ & $49 \%$ & $46 \%$ \\
\hline 6 & $57 \%$ & $57 \%$ & $55 \%$ & $51 \%$ \\
\hline 7 & $65 \%$ & $62 \%$ & $60 \%$ & $58 \%$ \\
\hline 12 & $93 \%$ & $87 \%$ & $94 \%$ & $83 \%$ \\
\hline
\end{tabular}

From the table it is clear that the analysis is quite sensitive to the points used to define the center of the circle. Skipping too few points can lead to large fluctuations in the radius of curvature between neighboring points, due to local variations in body geometry. However, skipping more points, as in Figure 23, also can provide unrealistic answers, as small radii of curvature are calculated even for points that are fairly linear, such as those contained in the oval in the figure.

Therefore, it was decided that a reasonable compromise between too few and too many points skipped would be to skip 5\% of the total points for each subject. Based on the 64 points used in each of Subject's 1 cross-sections, that meant 3 points were skipped for the calculation of the local radius of curvature. To put this conclusion another way, Figure 22 was considered a more accurate representation of the human body than Figure 23.

While the table above gives a rough idea of what the distribution is for each subject, a much more intuitive view of the data is to display each of the subject's legs with the local pressures (all readings in $\mathrm{kPa}$ ) as in the following figures.

Due to the nature of the data samples, in which data points are not always placed directly above another, sometimes there are large gaps, which can lead to an overabundance of one color due to the interpolation used to create these graphs. A good example of this is the large blue spot (enclosed in the red circle) in Figure 25 that is surrounded by some darker areas. The lack of the black vertical lines shows that the points did not align vertically, and so that area was created by interpolation. View 1 looks at the anterior and exterior portion of the leg, while view 2 displays the posterior and interior section. 


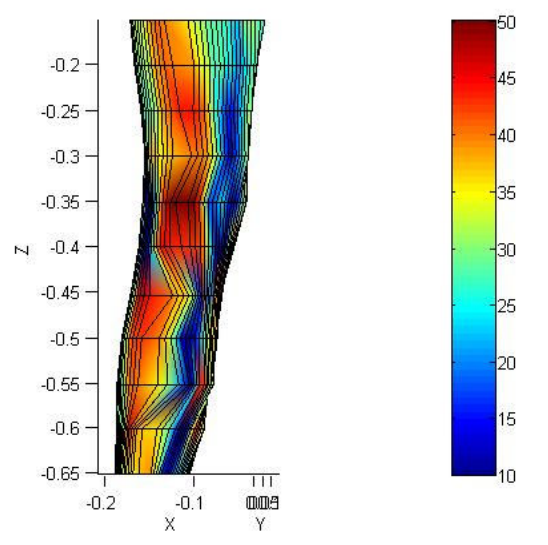

Figure 24: Cyberware Subject 1 view 1

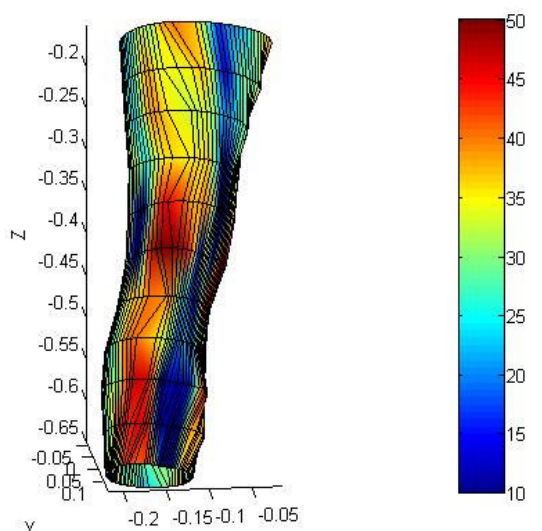

Figure 26: Cyberware Subject 2 view 1

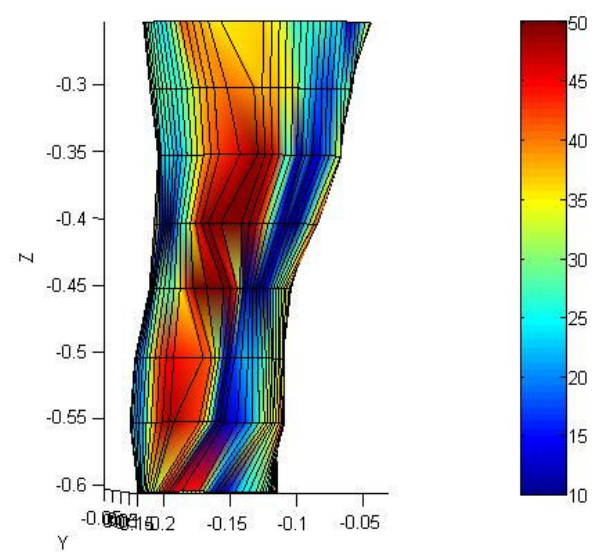

Figure 28: Cyberware Subject 3 view 1

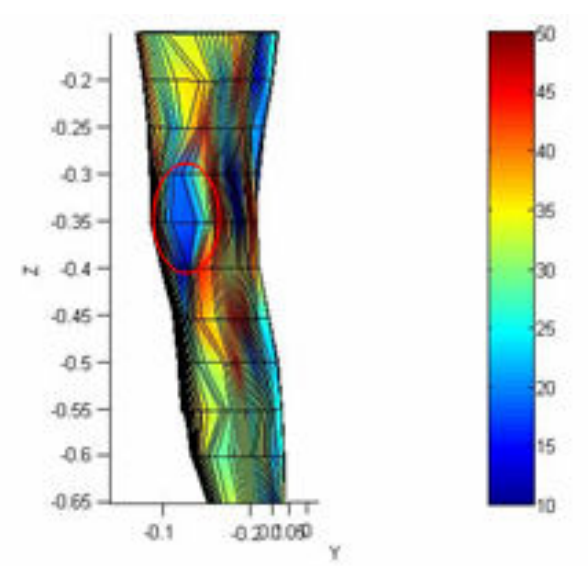

Figure 25: Cyberware Subject 1 view 2

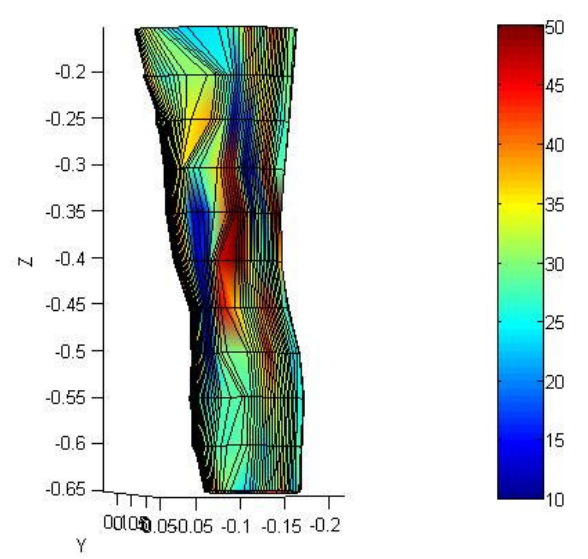

Figure 27: Cyberware Subject 2 view 2

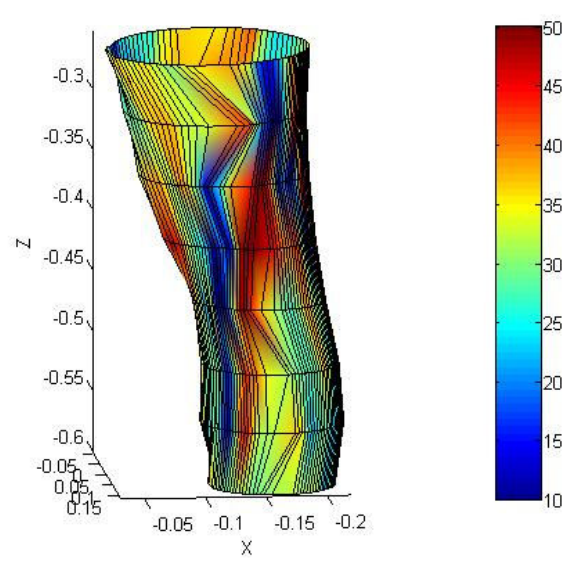

Figure 29: Cyberware Subject 3 view 2 


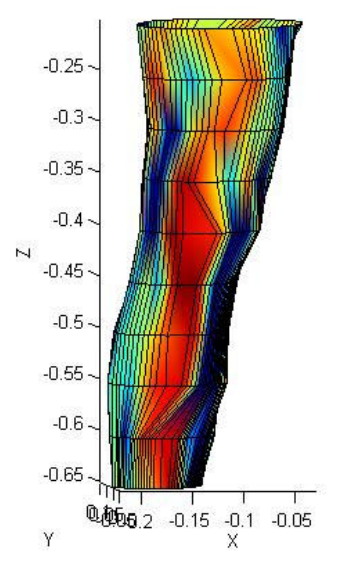

Figure 30: Cyberware Subject 4 view 1

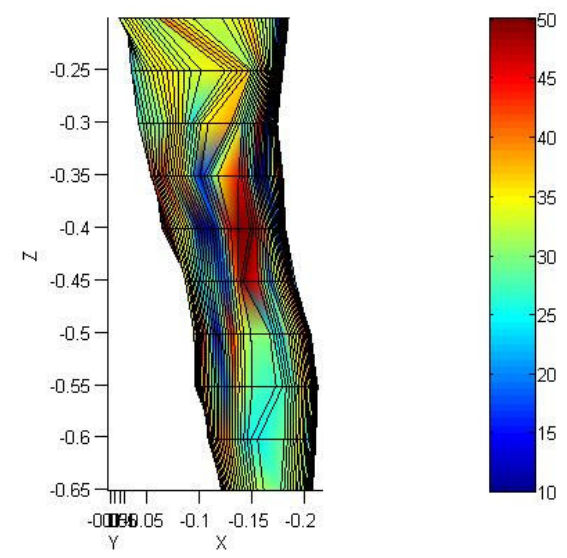

Figure 31: Cyberware Subject 4 view 2

\subsubsection{Discussion}

There are distinct patterns (stripes) that rise up the leg where pressure seems to be higher or lower. The posterior of the calf and the upper thigh, which are both typically quite fleshy, are approximately the desired pressure, $30 \mathrm{kPa}$. These results match quite well with the human testing conducted. The hard areas, such as the shin or the bone around the knee, do not deform easily and have concavities that are not easily pressurized. However, radii of curvature for the softer, rounded parts of the body are much closer to the average cross-sectional radius of curvature.

One major point is that even using "un-deformed" circumferences of the body measured under only atmospheric pressure, as opposed to more accurate measurements from a body "deformed" due to mechanical counterpressure, around $40 \%$ of the leg would be within $5 \mathrm{kPa}$ of the desired $30 \mathrm{kPa}$. This result should be considered encouraging. As noted previously in this document, the current (conservative) target is $30 \mathrm{kPa} \pm 1.6 \mathrm{kPa}$. Although pressure variation within that $1.6 \mathrm{kPa}$ range has been found to be acceptable [16], there is considerable research that needs to be conducted in this area to find a more definitive (possibly wider) range. In addition, when the body is placed under $\mathrm{MCP}$, the body deforms to balance out the pressures, although how much change in the body is created by MCP is currently unknown, since there is no scanning data with the leg under MCP available.

The initial MCP tests of Webb and Annis mentioned slight edema in certain flat or concave body sections, but indicated that subjects could tolerate up to twenty minutes of complete body enclosure in a MCP garment exposed to a vacuum [1,2]. Therefore, $\pm 5 \mathrm{kPa}$ provided a reasonable (probably conservative) range for comparison, since these unknowns should together increase the range of acceptable pressure variation.

\subsection{Conclusions}

Although there is considerable medical literature concerning effects of localized overpressure and underpressure, circumferential pressure variation is not a common condition and is not described in 
the medical literature. Therefore, human tolerance to circumferential pressure variations around the limbs must be determined experimentally.

Until this experimental testing has been completed, it is impossible to state with absolute certainty whether a spacesuit designed based on the concept of MCP would be safe for astronauts. However, the analysis certainly supports MCP as a viable replacement to gas-pressurized spacesuits, given the significant percentage of the human body that would receive pressure within an acceptable variation from $30 \mathrm{kPa}$. Therefore, further theoretical modeling work, with data sets based on subjects experiencing MCP, is warranted. 


\section{Chapter 4: TekScan testing}

\subsection{Motivation}

Significant effort was expended previously in the search for a system to measure the pressure produced by the BioSuit, since without that data it is impossible to know if the design meets the pressure targets stated in Chapter 2. The final choice for the pressure sensing system was from the TekScan company, specifically Model 9801 with the I Scan software ${ }^{11}$. However, when used in Sim's experiments, the system measured a lower pressure $(179 \mathrm{~mm} \mathrm{Hg})$ than expected $(225 \mathrm{~mm}$ $\mathrm{Hg}$ ) across the anterior of the shin. He reasoned that since the shin has areas of concavity due to its bony structure, the pressure was higher on convex portions of the lower leg (such as the calf), since the experimental subjects experienced little to no edema $[14]^{12}$.

As testing commenced as part of the full experimental study that will be outlined in Chapter 6 , it was discovered that the TekScan pressure sensors were not reading pressures that could be considered realistic. For example, the following table details the pressure measurements on Subject 2 who was in the low pressure chamber at $-225 \mathrm{~mm} \mathrm{Hg}$ for 36 minutes with minimal discomfort before a hardware failure unrelated to the pressure production ended the test. These readings are taken from the exterior of the thigh, which is a much softer portion of the body than the shin.

Table 16: Subject 2 pressure readings while experiencing $-225 \mathrm{~mm} \mathrm{Hg}$

\begin{tabular}{|c|c|c|}
\hline Time spent in chamber & Average pressure (mm Hg) & $\begin{array}{c}\text { Standard deviation } \\
\text { (mm Hg) }\end{array}$ \\
\hline Immediate $(0 \mathrm{~min})$ & 90 & 39 \\
\hline $5 \mathrm{~min}$ & 84 & 37 \\
\hline $10 \mathrm{~min}$ & 94 & 55 \\
\hline $15 \mathrm{~min}$ & 84 & 44 \\
\hline $20 \mathrm{~min}$ & 94 & 40 \\
\hline $30 \mathrm{~min}$ & 74 & 38 \\
\hline
\end{tabular}

\footnotetext{
${ }^{11}$ This system was also the choice of the Tanaka group mentioned in Ch. 2.

12 The choice of the sensors is an entire chapter in Sim's thesis (Ch. 3), while the analysis of the lower pressure on the shin is seen on page 110 of that document.

${ }^{13}$ The standard deviation was calculated using an unbiased estimator, $s=\sqrt{\frac{1}{N-1} \sum_{i=1}^{N}(x-\bar{x})^{2}}$ where $\mathrm{N}$ was the

number of readings, $\mathrm{x}$ was an individual reading, and $\bar{x}$ was the average pressure value. All readings of $0 \mathrm{~mm} \mathrm{Hg}$ were removed from the analysis prior to the calculation, as they were considered inaccurate. Three of the electrical connections in the handle that read the pressure of an individual sensel do not work properly, and always record 0 mm $\mathrm{Hg}$ of pressure. An unbiased estimator was used since the TekScan sensor covers only a small portion of the leg, and, therefore, is a part of a larger data set. The maximum number of data points is 96 , the number of sensels on the sensor, but most of the data sets consist of 90-93 points. If a larger number of data points was removed, that removal is noted in the text in this chapter.
} 
Similar readings exist for other subjects in this pilot study. If these pressure measurements are to be accepted as accurate, then these subjects should have experienced considerable edema and discomfort given these pressure differences. However, little to no edema was evident in many cases, leading to the conclusion that the pressure readings of the TekScan system were reading less than the actual pressure. The purpose of this chapter is to outline the testing that was conducted to determine both the reason for the pressure measurement inaccuracies as well as a means to mitigate these errors.

A brief summary of the tests conducted as well as the final conclusions shall be presented here to help the reader more readily understand this chapter. Initial tests were conducted by calibrating the TekScan system on surfaces of varying hardness, and then using the neoprene wrap to create MCP on a cylinder. The initial conclusion was that any change between the hardness of the calibration surface and the testing surface would significantly affect the TekScan readings. More specifically, calibrating on a soft surface and testing on a hard surface caused the TekScan to report higher than expected pressures, while calibrating on a hard surface and testing on a soft surface caused the TekScan to report lower than expected pressures. Systematically, variables such as the pressure producing mechanism (MCP to hybrid bladder) and the material directly above the sensor (plastic to neoprene rubber) were changed to eliminate them as the source of the inconsistent TekScan readings. The final conclusion was unchanged from the initial finding that the hardness of the calibration and testing surfaces was critical in obtaining accurate readings. A brief study was then undertaken to discover what calibration surface was optimal for the human experiments discussed in Chapter 6.

\subsection{TekScan overview}

In order for the reader to fully understand the remainder of this chapter, the capabilities of the TekScan system must be outlined here.

Each of the Model 9801 TekScan sensors used for this project has 96 individual sensing elements, or sensels. Each of these sensels is a variable resistor in an electrical circuit, where the resistance in the circuit decreases as the pressure applied is increased. These sensors are very thin $(0.1 \mathrm{~mm})$ and therefore fit easily under the neoprene wrap used to create mechanical counterpressure, and take real-time measurements, unlike many other pressure measuring systems.

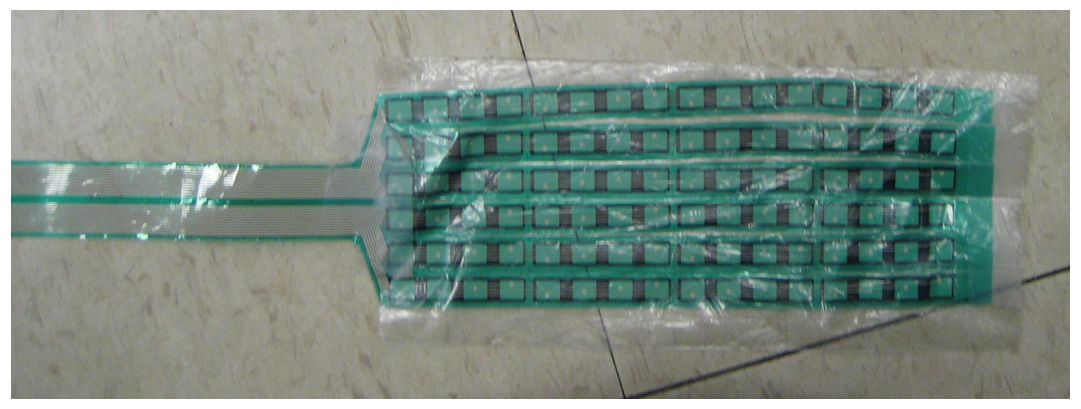

Figure 32: TekScan Model 9801 sensor 
However, the system does experience some inaccuracies, based on the information provided by the TekScan company [23], some of which will be outlined here.

- "Overall" system inaccuracy of $10 \%{ }^{14}$

- Creep of $3 \%$, depending on duration of applied load

- Hysteresis pressure change of up to $5 \%{ }^{15}$

- Hysteresis time of up to 3-5 minutes

- False pressure spikes when the sensor is crimped or wrinkled

Sim conducted tests in which a cylindrical and flat plate calibration devices were created in order to measure these properties of the TekScan system. A manometer is used to pressurize a plastic bladder which presses against the TekScan sensor, creating pressure [14]. The equipment used for the experiment can be seen in the figures below.

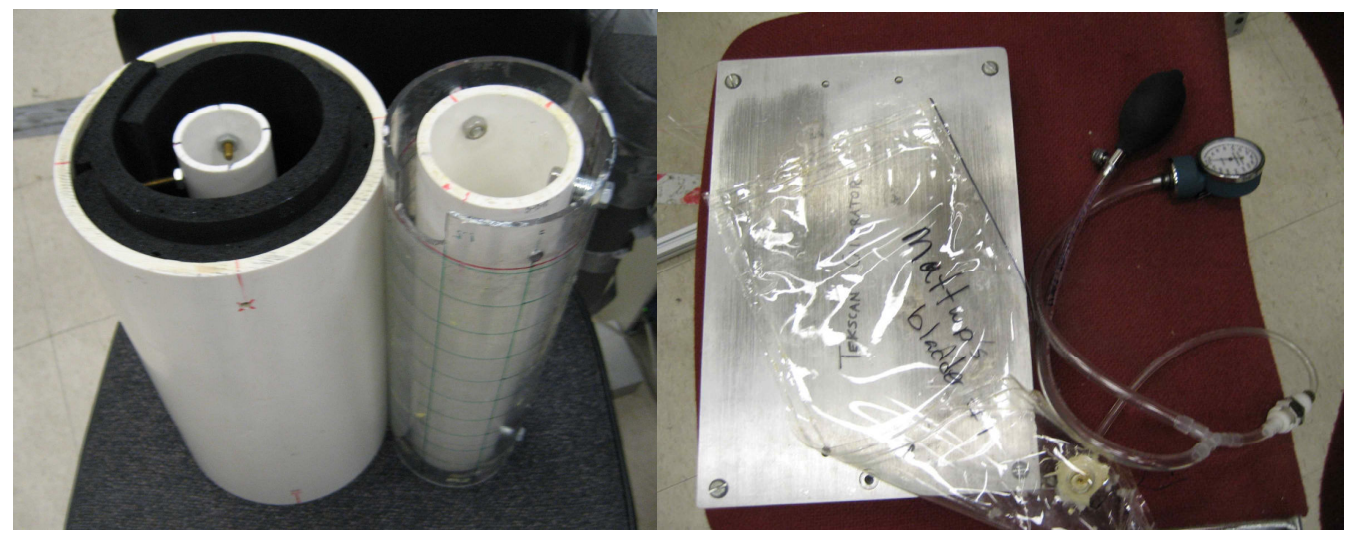

Figure 33: (left) Soft inner cylinder and hard cylinder used for calibration Figure 34: (right) Flat plate calibration device with the plastic bladder and the manometer

The experiments verified the limitations described above, although he also discovered that the hysteresis, while repeatable, was closer to $10 \%$ of the pressure being measured [14]. All of these values are larger than preferred, especially the "overall" inaccuracy of $10 \%$, but still does not account for the large variation that motivated the study.

\footnotetext{
14 The TekScan manual does not clearly define "overall."

${ }^{15}$ Again it is uncertain what this 5\% refers to.
} 


\subsection{TekScan procedure}

It is critical to understand the procedure for calibrating the sensor, as this affects the values that are recorded. This section provides a general description of the procedure, with a more detailed list of steps located in Appendix G.

When the TekScan sensor is received by the test conductor, a few steps must be taken to ensure the sensor is ready for usage. The 6 columns of sensels must be separated to give greater flexibility (which is critical for placing the sensors on the non-uniform human body) and then small holes are poked in each of the sensels to allow any air bubbles to escape, as otherwise they could cause improper readings. Plastic is then placed over the sensor, to protect it during testing. Throughout this process, great care is taken to ensure that none of the sensels are damaged. As 4 different sensors were used in the measurements in this chapter, it is unlikely that pre-processing activities were responsible for any errors in the system. However, these activities are noted as a possible, though highly unlikely, cause of the large variations in pressure between expected and measured values.

Before any testing can begin, the TekScan system must be calibrated using one of the two calibration rigs shown in the previous figures. First, the sensor is equilibrated, which means each of the sensels measures the same pressure (although the actual value is unknown yet). TekScan offers the ability to have 10 equilibration points, but only 6 are used for this process: 50, 100, 150, 200, 250 , and $300 \mathrm{~mm} \mathrm{Hg}$ (approximately). As this step is used simply to even the measurements, the exact pressure readings are somewhat irrelevant. It should be noted that a large leak in the bladder used to create pressure on the sensor can cause improper readings, based on the hysteresis mentioned previously. It is additionally possible that the manometer used to create pressure is the problem in this system as well, but the results in this chapter do not support this hypothesis, as will be noted in the text where appropriate.

The final step is to set 2 calibration points: 150 and $300 \mathrm{~mm} \mathrm{Hg}$ were always chosen in this thesis. More points would have been used, as TekScan uses an interpolation between these points to calibrate the sensel readings, but 2 is the maximum number of points allowed for calibration by the software.

The sensels were allowed to "settle" for 2 minutes before recording each calibration point to compensate for hysteresis effects, and great care was taken to measure exactly at 150 and $300 \mathrm{~mm}$ $\mathrm{Hg}$, otherwise the readings from the TekScan system would be incorrect.

Once calibration is completed, the sensor is ready to be used in testing.

\subsection{TekScan measurements}

In order to discover the reason for the variation in pressure readings, a number of tests were conducted using various calibration devices, the TekScan sensors, neoprene leg wraps, and even human subjects. 


\subsubsection{Initial tests of wrap}

The first set of tests consisted of wrapping the neoprene bands over uniform cylinders, to eliminate the non-uniform cross-sections of the body as the cause of the variation between expected and measured pressures.

An elastic binding wrap designed to produce $30 \mathrm{kPa}(225 \mathrm{~mm} \mathrm{Hg})$ by spiraling up a hard plastic cylinder was created (Figure 33), and the pressure it produced was tested using the TekScan system. The pressure readings (based off calibration in the flat plate) stabilized after 5 minutes close to the expected value. Figure 35 shows the neoprene wrap on the cylinder.

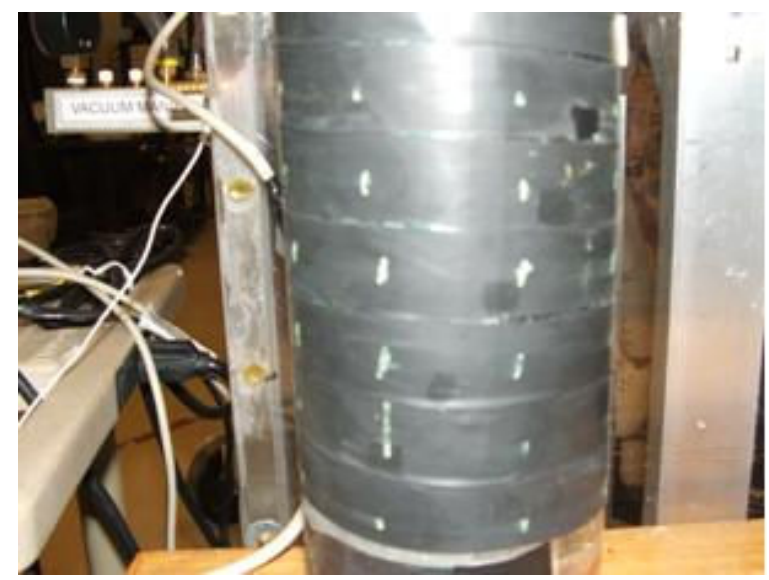

Figure 35: Close-up of wrap on hard cylinder

Table 17: Neoprene wrap over hard cylinder

\begin{tabular}{|c|c|c|c|c|}
\hline Time & $\begin{array}{c}\text { Test 1 measured } \\
\text { pressure } \\
(\mathrm{mm} \mathrm{Hg})\end{array}$ & $\begin{array}{c}\text { Standard } \\
\text { deviation } \\
(\mathrm{mm} \mathrm{Hg})\end{array}$ & $\begin{array}{c}\text { Test 2 measured } \\
\text { pressure } \\
(\mathrm{mm} \mathrm{Hg})\end{array}$ & $\begin{array}{c}\text { Standard } \\
\text { deviation } \\
(\mathrm{mm} \mathrm{Hg})\end{array}$ \\
\hline $\begin{array}{c}0 \text { min } \\
(\text { immediate })\end{array}$ & 219 & 39 & 221 & 44 \\
\hline $1 \mathrm{~min}$ & 222 & 39 & 222 & 46 \\
\hline $5 \mathrm{~min}$ & 225 & 39 & 229 & 47 \\
\hline
\end{tabular}

These tests indicate the manometer is accurately reading the pressure, assuming proper calibration of the band. Given the simplistic nature of Equation 1 and the uniform cross-section of the cylinder, this is likely. The lack of discomfort felt by subjects in the Sim experiment further reinforces this assumption as reasonable.

Additional tests were then undertaken on a "soft" cylinder, which consisted of a piece of foam surrounding a hard cylinder, to discover if the inaccuracies lay with not the wrap, but the surface upon which the wrap was placed. The soft outer cylinder was made using a piece of foam that will 
deform if placed under sufficient force, and therefore is a more reasonable approximation of human flesh than a hard plastic cylinder and can be seen in Figure 33. A piece of soft plastic identical to that used to cover the TekScan Model 9801 sensor was placed over the foam to create a smooth surface for superior calibration. The same piece of neoprene used for the previous wrap was used on the soft cylinder as well. The full set-up can be seen in Figure 36, with the hard cylinder previously described in the background. As seen in the figure, the wrap does not completely cover the sensor, which limits the number of data points obtained. ${ }^{16}$

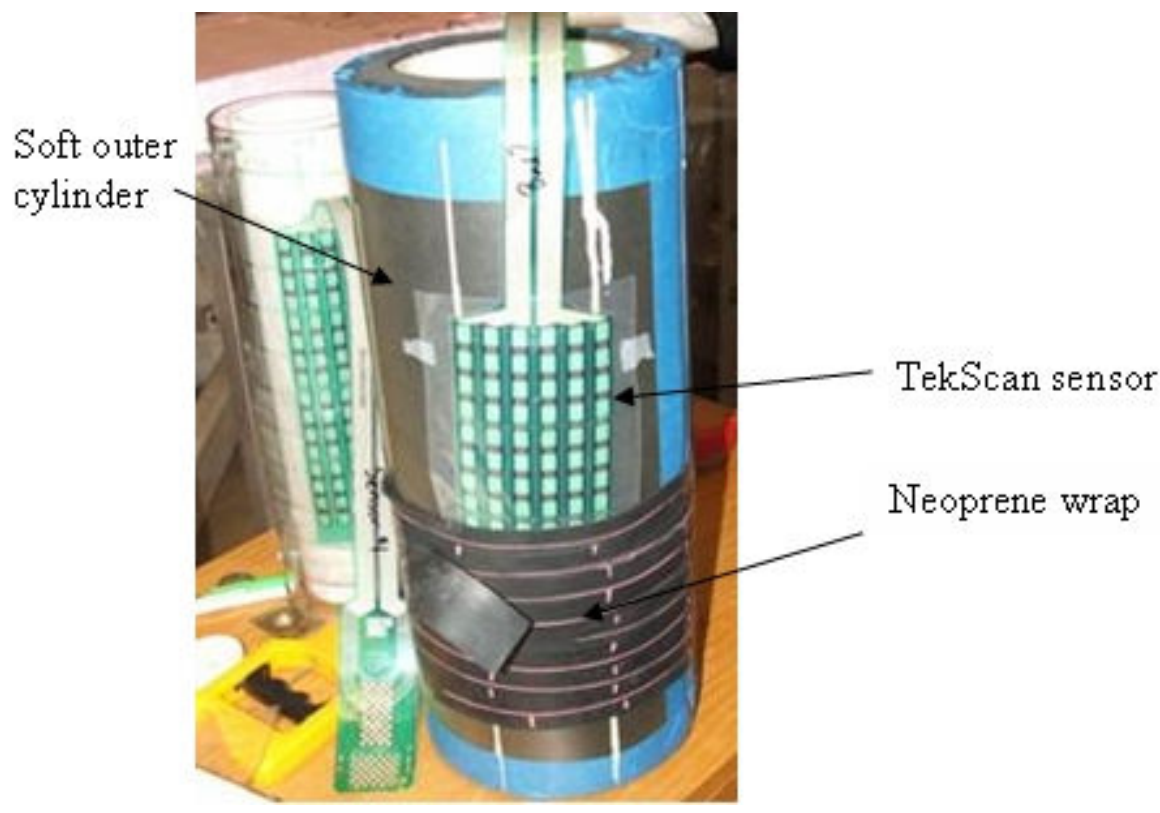

Figure 36: Wrap on cylinder with soft outer foam

The results are similar to those taken on the human body: significantly lower than expected. Admittedly the foam is not a perfect simulator for the human body, as the deformation under $30 \mathrm{kPa}$ of pressure was small (on the order of 1-2 $\mathrm{mm}$ ), but it certainly represents a much better representation than the hard cylinder. The results are displayed in Table 18.

\footnotetext{
${ }^{16}$ While the hard cylinder was completely covered by the neoprene wrap, this wrap over the soft cylinder covered it only partially. The reason for the difference was that a single wrap was used over the hard cylinder, whereas a double wrap created pressure on the soft outer cylinder. A single wrap uses one layer of material, while a double wrap requires two layers to create the desired $30 \mathrm{kPa}$ of pressure. The type of wrap was changed as at the same time a decision was made to use a double wrap in place of the single wrap for human testing. The use of additional material, as well as the larger radius of the soft cylinder, reduced the number of sensels that could be pressurized. Much more information about the neoprene wrap is found in Chapter 6 of this thesis.
} 
Table 18: Neoprene wrap over soft outer cylinder $^{17}$

\begin{tabular}{|c|c|c|c|c|c|c|}
\hline Time & $\begin{array}{c}\text { Test 1 } \\
\text { measured } \\
\text { pressure } \\
(\mathrm{mm} \mathrm{Hg})\end{array}$ & $\begin{array}{c}\text { Standard } \\
\text { deviation } \\
(\mathrm{mm} \mathrm{Hg})\end{array}$ & $\begin{array}{c}\text { Test 2 } \\
\text { measured } \\
\text { pressure } \\
(\mathrm{mm} \mathrm{Hg})\end{array}$ & $\begin{array}{c}\text { Standard } \\
\text { deviation } \\
(\mathrm{mm} \mathrm{Hg})\end{array}$ & $\begin{array}{c}\text { Test 3 } \\
\text { measured } \\
\text { pressure } \\
(\mathrm{mm} \mathrm{Hg})\end{array}$ & $\begin{array}{c}\text { Standard } \\
\text { deviation } \\
(\mathrm{mm} \mathrm{Hg})\end{array}$ \\
\hline $\begin{array}{c}0 \text { min } \\
\text { (immediate })\end{array}$ & 136 & 31 & 178 & 37 & 168 & 25 \\
\hline $1 \mathrm{~min}$ & 137 & 39 & 179 & 36 & 171 & 25 \\
\hline $5 \mathrm{~min}$ & 142 & 14.3 & 183 & 37 & 173 & 25 \\
\hline
\end{tabular}

The reason for the wide range of values is not clear, but reinforces the hypothesis that using the TekScan sensors on a soft surface, whether foam or human skin, leads to inaccuracies, as the measured pressure was at least $40 \mathrm{~mm} \mathrm{Hg}$ below the expected value.

Another test was conducted because it seemed likely that the pressure variation was caused not by the neoprene wrap, but rather by the change in hardness from the calibration to testing surfaces upon which the sensor was placed. This hypothesis was developed because when calibrating and testing the sensor on top of a hard surface (metal and plastic) the pressure measured was near the expected pressure, whereas the sensor measured the results seen in Table 18 when the surface underneath the sensor was changed. Therefore, an experiment was conducted in which the sensor was calibrated on the cylinder with foam inside (the soft "inner" cylinder), and then wrapped on the cylinder with foam around it (soft "outer" cylinder).

Table 19: Calibrated on soft inner cylinder, measured on soft wrapped cylinder

\begin{tabular}{|c|c|c|}
\hline Time & $\begin{array}{c}\text { Average pressure } \\
(\mathrm{mm} \mathrm{Hg})\end{array}$ & $\begin{array}{c}\text { Standard deviation } \\
(\mathrm{mm} \mathrm{Hg})\end{array}$ \\
\hline Immediate $(0 \mathrm{~min})$ & 207 & 38 \\
\hline $1 \mathrm{~min}$ & 212 & 38 \\
\hline $3 \mathrm{~min}$ & 214 & 38 \\
\hline
\end{tabular}

Again, this table shows the sensor hysteresis, but it also provides evidence that using the soft inner cylinder is a reasonable calibration device for the soft wrapped cylinder. One possible reason the pressure is not quite $225 \mathrm{~mm} \mathrm{Hg}$ could be because the band has been stretched many times and is beginning to fatigue, but this creep is considered unlikely, for reasons which will be made clear in Chapter 5.

\footnotetext{
${ }^{17}$ Since the sensor was not fully covered, only 42 sensels were pressurized, and that was the number used in the calculation of the standard deviation.
} 
The previous tests have indicated that a Model 9801 sensor moved from a hard calibration surface to a hard testing surface (henceforth, this shall be referred to as "hard-to-hard" testing, with the hardness of the calibration surface followed by the hardness of the testing surface, in order to reduce the use of redundant text) and "soft-to-soft' testing measures the expected results. However, the TekScan sensor in "hard-to-soft" testing provided very low pressure readings. It was considered possible that the results of this "hard-to-soft" testing were due to the change from flat calibration to cylindrical testing, although the results displayed in Table 17 do not support this theory. In order to be sure, however, tests were conducted using "hard-to-soft" and "soft-to-hard" cylindrical testing to remove geometry changes as a reason for the measured pressure variation.

The data in Table 20 shows the differences between the expected and measured pressure when the calibration is conducted on the cylinder of opposite hardness. As mentioned before, the expected pressure for all the wrapped measurements is $225 \mathrm{~mm} \mathrm{Hg}$.

Table 20: Wrapped cylinders with opposing calibrations

\begin{tabular}{|c|c|c|c|c|}
\hline Time & $\begin{array}{c}\text { Soft outer cylinder } \\
\text { wrapped, calibrated } \\
\text { on hard }(\mathrm{mm} \mathrm{Hg})\end{array}$ & $\begin{array}{c}\text { Standard } \\
\text { deviation } \\
(\mathrm{mm} \mathrm{Hg})\end{array}$ & $\begin{array}{c}\text { Hard outer cylinder } \\
\text { wrapped, calibrated } \\
\text { on soft }(\mathrm{mm} \mathrm{Hg})\end{array}$ & $\begin{array}{c}\text { Standard } \\
\text { deviation } \\
(\mathrm{mm} \mathrm{Hg})\end{array}$ \\
\hline Immediate & 128 & 32 & 422 & 122 \\
\hline $1 \mathrm{~min}$ & 135 & 31 & 427 & 122 \\
\hline $3 \mathrm{~min}$ & 138 & 30 & 433 & 119 \\
\hline
\end{tabular}

The data strongly suggests that the surface upon which the TekScan was placed was causing the difference in expected and measured pressure values. More specifically, pressure measurements from a "soft-to-hard" test are significantly higher than expected results, while a "hard-to-soft" test produces TekScan readings much lower than anticipated. However, it was considered possible that the neoprene wrap was still affecting the results in some form. Therefore, in order to eliminate the neoprene wrap as the source of the error, another set of experiments was conducted.

\subsubsection{Testing without neoprene wrap}

These tests were conducted in order to ensure that the problem lay indeed with the change in surfaces upon which the TekScan was being used, as now the surface "above" the sensor (the surface producing the pressure), was held constant. If the measurements differed when calibrated and tested in the same device, then the problem must lie with the TekScan sensor, given that all the other possibilities have been eliminated. Instead of the neoprene bands, a bladder was used to pressurize the bands, and details on the construction of the bladders can be seen in Appendix $\mathrm{H}$.

\subsubsection{Calibration and testing in same device}

The experiments conducted in this section show clearly that calibrating and testing on the same surface allows for accurate TekScan measurements. All 3 of the calibration devices: the flat plate, the hard cylinder, and the cylinder with the soft inner foam lining, were utilized to ensure that the variation is independent both of the calibration surface hardness and geometry. 
The first test was conducted in the flat plate using a layer of foam underneath the TekScan (as opposed to the bottom metal plate), as in Figure 37.

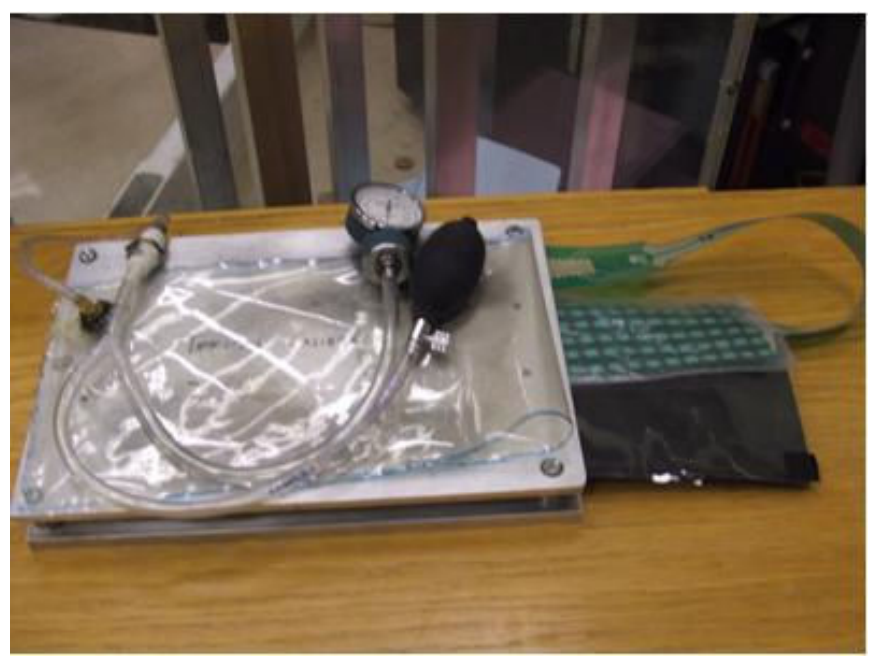

Figure 37: Equipment for flat plate measurements

The flat plate was used since it allows the sensor to lie horizontally, rather than being forced to fit in a narrow cylinder, and is, therefore, much less likely to crinkle the sensor, causing unwanted pressure spikes. The following data (Table 21) was collected.

Table 21: Flat plate measurements (calibrated and measured on piece of foam)

\begin{tabular}{|c|c|c|}
\hline $\begin{array}{c}\text { Gauge pressure } \\
(\mathrm{mm} \mathrm{Hg})\end{array}$ & $\begin{array}{c}\text { TekScan measurement } \\
(\mathrm{mm} \mathrm{Hg})\end{array}$ & $\begin{array}{c}\text { Standard deviation } \\
(\mathrm{mm} \mathrm{Hg})\end{array}$ \\
\hline 150 & 152 & 13 \\
\hline 200 & 200 & 13 \\
\hline 220 & 219 & 13 \\
\hline 240 & 242 & 19 \\
\hline 300 & 305 & \\
\hline
\end{tabular}

The pressures are obviously quite close together, supporting the hypothesis that a surface change between calibration and testing is the reason for pressure variation. The following tables display the multiple tests conducted with the hard cylinder and the cylinder with the soft inner foam. 
Table 22: Pressure measurements on hard cylinder (calibrated and measured)

\begin{tabular}{|c|c|c|c|c|}
\hline $\begin{array}{c}\text { Gauge } \\
\text { pressure } \\
(\mathrm{mm} \mathrm{Hg})\end{array}$ & $\begin{array}{c}\text { Test 1 } \\
(\mathrm{mm} \mathrm{Hg})\end{array}$ & $\begin{array}{c}\text { Standard } \\
\text { deviation } \\
(\mathrm{mm} \mathrm{Hg})\end{array}$ & $\begin{array}{c}\text { Test 2 } \\
(\mathrm{mm} \mathrm{Hg})\end{array}$ & $\begin{array}{c}\text { Standard } \\
\text { deviation } \\
(\mathrm{mm} \mathrm{Hg})\end{array}$ \\
\hline 150 & 141 & 13 & 126 & 23 \\
\hline 200 & 192 & 16 & 180 & 26 \\
\hline 220 & 214 & 16 & 206 & 30 \\
\hline 240 & 238 & 16 & 227 & 32 \\
\hline 300 & 284 & 19 & 284 & 32 \\
\hline
\end{tabular}

Table 23: Pressure measurements on cylinder with soft inner foam (calibrated and measured)

\begin{tabular}{|c|c|c|c|c|}
\hline $\begin{array}{c}\text { Gauge } \\
\text { pressure } \\
(\mathrm{mm} \mathrm{Hg})\end{array}$ & $\begin{array}{c}\text { Test 1 } \\
(\mathrm{mm} \mathrm{Hg})\end{array}$ & $\begin{array}{c}\text { Standard } \\
\text { deviation } \\
(\mathrm{mm} \mathrm{Hg})\end{array}$ & $\begin{array}{c}\text { Test 2 } \\
(\mathrm{mm} \mathrm{Hg})\end{array}$ & $\begin{array}{c}\text { Standard } \\
\text { deviation } \\
(\mathrm{mm} \mathrm{Hg})\end{array}$ \\
\hline 150 & 151 & 13 & 142 & 13 \\
\hline 200 & 208 & 13 & 199 & 13 \\
\hline 220 & 230 & 13 & 222 & 14 \\
\hline 240 & 251 & 13 & 245 & 14 \\
\hline 300 & 302 & 16 & 310 & 18 \\
\hline
\end{tabular}

While the greatest accuracy is near the outlying calibration points, it is important to ensure that all the pressure measurements match gauge readings. The value of $220 \mathrm{~mm} \mathrm{Hg}$ was chosen since it was close to $225 \mathrm{~mm} \mathrm{Hg}$, the goal for the full leg test, and much easier to read off the gauge than the higher number. The 200 and $240 \mathrm{~mm} \mathrm{Hg}$ points were used as brackets around the desired pressure value: far enough away to record a different value, but close enough to ensure that one accurate reading at $220 \mathrm{~mm} \mathrm{Hg}$ would not be a fluke.

The differences between the expected and measured pressure are much larger (up to $20 \mathrm{~mm} \mathrm{Hg}$ in Table 22), but some of this variation may be caused by crinkling of the sensor in the cylinders. However, these readings are still close to the expected, and not anywhere near the large variations that motivated this study.

\subsubsection{Soft vs. hard cylinder and flat plate testing}

It has been shown that testing and calibrating in the same device causes small to no variation in TekScan readings. The testing outlined in this section is meant to show that changes in surface between calibration and testing does cause significant variation.

Two tests were conducted with calibration on the hard plastic inside the flat plate, then measurements taken on the foam inside the flat plate. 
Table 24: Flat plate measurements

\begin{tabular}{|c|c|c|c|c|}
\hline $\begin{array}{c}\text { Gauge } \\
\text { pressure } \\
(\mathrm{mm} \mathrm{Hg})\end{array}$ & $\begin{array}{c}\text { TekScan } \\
\text { Measurement \#1 } \\
(\mathrm{mm} \mathrm{Hg})\end{array}$ & $\begin{array}{c}\text { Standard } \\
\text { deviation } \\
(\mathrm{mm} \mathrm{Hg})\end{array}$ & $\begin{array}{c}\text { TekScan } \\
\text { Measurement \#2 } \\
(\mathrm{mm} \mathrm{Hg})\end{array}$ & $\begin{array}{c}\text { Standard } \\
\text { deviation } \\
(\mathrm{mm} \mathrm{Hg})\end{array}$ \\
\hline 150 & 129 & 26 & 117 & 19 \\
\hline 200 & 181 & 29 & 167 & 21 \\
\hline 220 & 205 & 31 & 188 & 22 \\
\hline 240 & 228 & 34 & 208 & 23 \\
\hline 300 & 286 & 42 & 267 & 20 \\
\hline
\end{tabular}

Although the differences vary between the two tests (for an unknown reason) it is clear that the readings are affected by the change in surface from the hard plastic inside the flat test plate to the foam.

For further evidence of the nature of these results, two additional tests were conducted on the cylinders. The first test was "hard-to-soft" and the second was "soft-to hard" cylindrical testing, the results of which are seen in the following tables.

Table 25: Calibrated on hard cylinder, measured on soft cylinder

\begin{tabular}{|c|c|c|c|c|c|c|}
\hline $\begin{array}{c}\text { Gauge } \\
\text { pressure } \\
(\mathrm{mm} \mathrm{Hg})\end{array}$ & $\begin{array}{c}\text { Test 1 } \\
(\mathrm{mm} \mathrm{Hg})\end{array}$ & $\begin{array}{c}\text { Standard } \\
\text { deviation } \\
(\mathrm{mm} \mathrm{Hg})\end{array}$ & $\begin{array}{c}\text { Test 2 } \\
(\mathrm{mm} \mathrm{Hg})\end{array}$ & $\begin{array}{c}\text { Standard } \\
\text { deviation } \\
(\mathrm{mm} \mathrm{Hg})\end{array}$ & $\begin{array}{c}\text { Test 3 } \\
(\mathrm{mm} \mathrm{Hg})\end{array}$ & $\begin{array}{c}\text { Standard } \\
\text { deviation } \\
(\mathrm{mm} \mathrm{Hg})\end{array}$ \\
\hline 150 & 116 & 15 & 111 & 15 & 98 & 12 \\
\hline 200 & 162 & 18 & 156 & 16 & 148 & 13 \\
\hline 220 & 184 & 19 & 179 & 18 & 170 & 16 \\
\hline 240 & 204 & 17 & 199 & 21 & 188 & 20 \\
\hline 300 & 266 & 27 & 255 & 26 & 248 & 21 \\
\hline
\end{tabular}

Again, there is a wide range of data points, but all of the pressure readings are far lower than expected. 
Table 26: Calibrated on soft cylinder, measured on hard cylinder

\begin{tabular}{|c|c|c|c|c|c|c|}
\hline $\begin{array}{c}\text { Gauge } \\
\text { pressure } \\
(\mathrm{mm} \mathrm{Hg})\end{array}$ & $\begin{array}{c}\text { Test 1 } \\
(\mathrm{mm} \mathrm{Hg})\end{array}$ & $\begin{array}{c}\text { Standard } \\
\text { deviation } \\
(\mathrm{mm} \mathrm{Hg})\end{array}$ & $\begin{array}{c}\text { Test 2 } \\
(\mathrm{mm} \mathrm{Hg})\end{array}$ & $\begin{array}{c}\text { Standard } \\
\text { deviation } \\
(\mathrm{mm} \mathrm{Hg})\end{array}$ & $\begin{array}{c}\text { Test 3 } \\
(\mathrm{mm} \mathrm{Hg})\end{array}$ & $\begin{array}{c}\text { Standard } \\
\text { deviation } \\
(\mathrm{mm} \mathrm{Hg})\end{array}$ \\
\hline 150 & 184 & 21 & 159 & 20 & 174 & 19 \\
\hline 200 & 253 & 27 & 220 & 23 & 241 & 22 \\
\hline 220 & 286 & 29 & 255 & 26 & 270 & 24 \\
\hline 240 & 312 & 35 & 286 & 30 & 298 & 27 \\
\hline 300 & 391 & 39 & 367 & 38 & 377 & 34 \\
\hline
\end{tabular}

As can be seen from these tables, when a TekScan sensor is calibrated on the hard cylinder and then placed on a softer surface, it records much lower pressures than expected. The sensor readings are based on the expectation of a hard surface, but when the surface deforms, it is unable to adjust. For the reverse test (soft-to-hard), the readings are much higher than they should be. The soft calibration means the sensor readings expect the surface to provide some displacement, and when it does not, the reading consequently increases significantly. Interestingly, the differences from the actual values are almost constant when calibrated on the hard cylinder and measured on the soft cylinder, but they increase proportionally with the applied pressure when calibrated on the soft cylinder and measured on the hard cylinder.

From this evidence it appears clear that surface upon which the TekScan is calibrated is the key source of error.

\subsubsection{Testing of seal material}

After determining that the surface under the sensor is important to the accuracy of the measurements obtained, the material above the sensor was considered. At one point, the seal design (a device that separates the vacuum chamber from the atmosphere, which will be covered in more depth in Chapter 5) consisted of a neoprene neck gasket and a neoprene knee brace. This brace was placed on top of the TekScan sensor on the leg, and since it has been shown quite clearly that a difference in calibration to testing surfaces under the sensor cause pressure variations, it seems wise to investigate the possibility that the material above the sensor causes the same inaccuracies.

Two different experiments were run, using both a flat metal plate with a layer of foam and the soft cylinder. First, the sensor was calibrated in the flat plate with the foam. Then a portion of the seal was cut into a small strip, as seen in Figure 38, and was placed on top of the sensor inside the flat plate. 


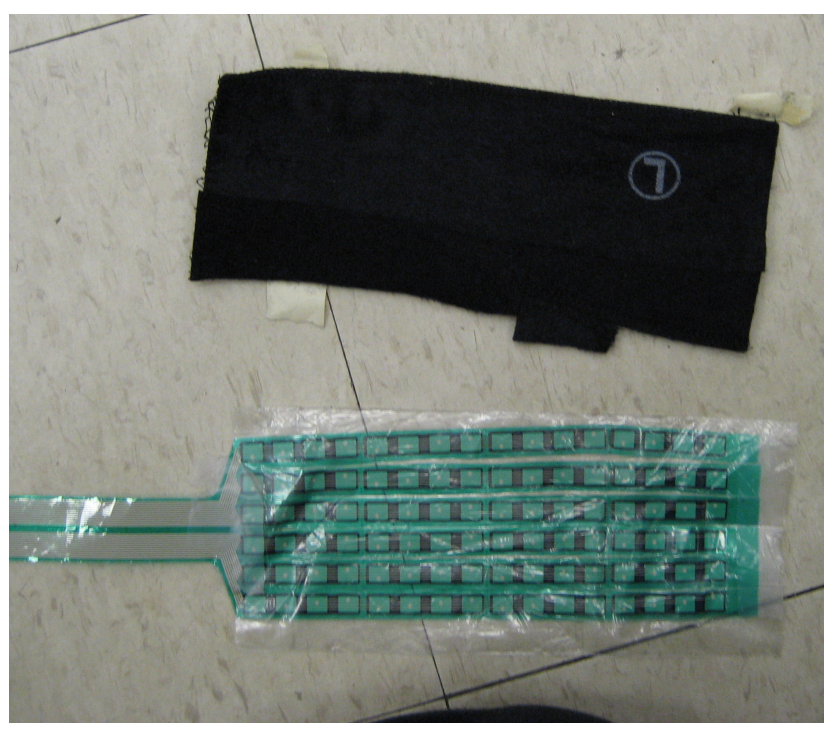

Figure 38: Seal and TekScan sensor

The following table details the results of the testing within the flat plate, based on calibration without the seal material, and then testing with it.

Table 27: Calibrated on flat plate, measured on flat plate with seal ${ }^{18}$

\begin{tabular}{|c|c|c|c|c|c|c|}
\hline $\begin{array}{c}\text { Gauge } \\
\text { pressure } \\
(\mathrm{mm} \mathrm{Hg})\end{array}$ & $\begin{array}{c}\text { Test 1 } \\
(\mathrm{mm} \mathrm{Hg})\end{array}$ & $\begin{array}{c}\text { Standard } \\
\text { deviation } \\
(\mathrm{mm} \mathrm{Hg})\end{array}$ & $\begin{array}{c}\text { Test 2 } \\
(\mathrm{mm} \mathrm{Hg})\end{array}$ & $\begin{array}{c}\text { Standard } \\
\text { deviation } \\
(\mathrm{mm} \mathrm{Hg})\end{array}$ & $\begin{array}{c}\text { Test 3 } \\
(\mathrm{mm} \mathrm{Hg})\end{array}$ & $\begin{array}{c}\text { Standard } \\
\text { deviation } \\
(\mathrm{mm} \mathrm{Hg})\end{array}$ \\
\hline 150 & 135 & 20 & 130 & 16 & 135 & 16 \\
\hline 200 & 186 & 22 & 182 & 22 & 186 & 20 \\
\hline 220 & 209 & 24 & 207 & 22 & 227 & 24 \\
\hline 240 & 230 & 25 & 231 & 23 & 237 & 23 \\
\hline 300 & 292 & 26 & 306 & 29 & 313 & 26 \\
\hline
\end{tabular}

Although the measured values are slightly different from the actual values, they are mostly within $15 \mathrm{~mm} \mathrm{Hg}$ of the gauge pressure, which is within the $10 \%$ boundary specified by TekScan. Typically, as the pressure increases, the difference from the gauge pressure either remains constant or changes linearly.

The same test was run using the soft cylinder because it was thought that the soft cylinder was more similar to human skin. The results had more inconsistencies within the sensels, possibly because the foam allowed the sensor to fold or move more readily. The results with all the data points included

\footnotetext{
${ }^{18}$ The values for test 3 were edited because part of one column of sensels on the sensor gave excessively high values (up to $100 \mathrm{~mm} \mathrm{Hg} \mathrm{higher} \mathrm{than} \mathrm{expected),} \mathrm{so} \mathrm{these} \mathrm{values} \mathrm{were} \mathrm{removed.} \mathrm{The} \mathrm{most} \mathrm{likely} \mathrm{cause} \mathrm{is} \mathrm{the} \mathrm{TekScan} \mathrm{sensor}$ folding over the edge of the seal.
} 
show more variation than when the flat plate was used, but the obtained measurements are still not considerably different from the actual values.

Table 28: Calibrated on soft cylinder, measured on soft cylinder with seal ${ }^{19}$

\begin{tabular}{|c|c|c|c|c|c|c|}
\hline $\begin{array}{c}\text { Gauge } \\
\text { pressure } \\
(\mathrm{mm} \mathrm{Hg})\end{array}$ & $\begin{array}{c}\text { Test 1 } \\
(\mathrm{mm} \mathrm{Hg})\end{array}$ & $\begin{array}{c}\text { Standard } \\
\text { deviation } \\
(\mathrm{mm} \mathrm{Hg})\end{array}$ & $\begin{array}{c}\text { Test 2, } \\
\text { excluding } \\
\text { column } \\
(\mathrm{mm} \mathrm{Hg})\end{array}$ & $\begin{array}{c}\text { Standard } \\
\text { deviation } \\
(\mathrm{mm} \mathrm{Hg})\end{array}$ & $\begin{array}{c}\text { Test 3, } \\
\text { excluding } \\
\text { column } \\
(\mathrm{mm} \mathrm{Hg})\end{array}$ & $\begin{array}{c}\text { Standard } \\
\text { deviation } \\
(\mathrm{mm} \mathrm{Hg})\end{array}$ \\
\hline 150 & 128 & 22 & 118 & 22 & 145 & 22 \\
\hline 200 & 178 & 28 & 170 & 27 & 198 & 24 \\
\hline 220 & 204 & 30 & 194 & 29 & 222 & 26 \\
\hline 240 & 228 & 35 & 219 & 32 & 243 & 28 \\
\hline 300 & 296 & 45 & 287 & 38 & 309 & 33 \\
\hline
\end{tabular}

It is much harder to get the sensor into the soft cylinder without bending it than into the flat plate and this may have caused the increase in pressure along the edge.

In order to test the opposite phenomenon, a test was run in which the seal was included during the calibration, but then the pressure was measured without the seal present. These values further suggest that the seal does not significantly affect the results.

Table 29: Calibrated with seal, measured without seal

\begin{tabular}{|c|c|c|c|c|}
\hline $\begin{array}{c}\text { Gauge } \\
\text { pressure } \\
(\mathrm{mm} \mathrm{Hg})\end{array}$ & $\begin{array}{c}\text { Flat plate } \\
(\mathrm{mm} \mathrm{Hg})\end{array}$ & $\begin{array}{c}\text { Standard } \\
\text { deviation } \\
(\mathrm{mm} \mathrm{Hg})\end{array}$ & $\begin{array}{c}\text { Soft } \\
\text { cylinder } \\
(\mathrm{mm} \mathrm{Hg})\end{array}$ & $\begin{array}{c}\text { Standard } \\
\text { deviation } \\
(\mathrm{mm} \mathrm{Hg})\end{array}$ \\
\hline 150 & 139 & 21 & 143 & 23 \\
\hline 200 & 191 & 25 & 197 & 28 \\
\hline 220 & 219 & 26 & 226 & 31 \\
\hline 240 & 242 & 27 & 249 & 32 \\
\hline 300 & 304 & 33 & 317 & 43 \\
\hline
\end{tabular}

Overall, the experiments with the seal show that although the values vary somewhat with the seal, they are still close to the actual pressures. Therefore, it can be concluded that the material above the sensor, in this case the seal, does not significantly affect the readings from the TekScan system.

\subsubsection{Human testing}

The motivation behind this study was how to best use the TekScan system for the desired application of measuring mechanical counterpressure on the human body. Therefore, the data

\footnotetext{
${ }^{19}$ Again one column was removed due to very high readings, probably for the same reason described in the previous footnote.
} 
collected previously in this chapter was used to design a series of experiments to understand how the TekScan system could be both tested and calibrated on a human body.

\subsubsection{Human skin testing with bladder}

In a final attempt to show the extreme sensitivity of the TekScan system to the surface upon which it is placed, two more attempts were conducted. Once again the calibrations were completed on a hard cylinder and then a soft cylinder, but this time measurements were taken on human skin, the anterior of the thigh to be exact. A hard plastic cylinder, similar to, but larger than, the outer shell of the hard cylinder seen in Figure 33, was placed over the subject's leg and then the TekScan sensor and bladder were inserted. The following figures show the experimental set-up and a diagram to clarify the design.
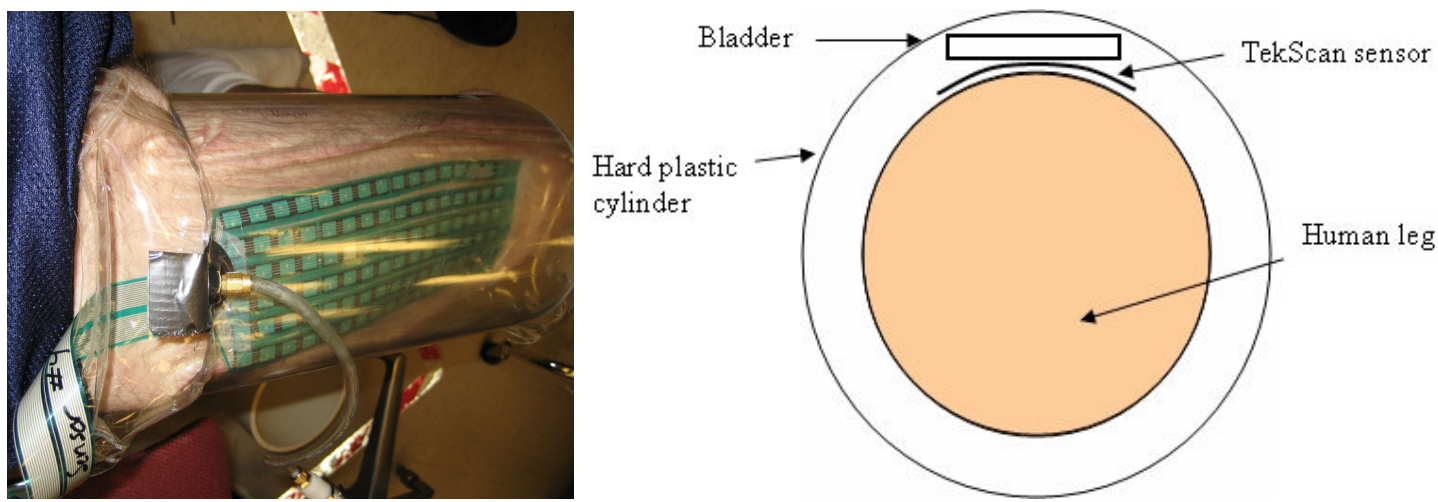

Figure 39: (left) Experimental set-up for human TekScan testing

Figure 40: (right) Diagram of experimental set-up

This test caused a fair amount of subject discomfort, as can be seen in the places on the leg that were compressed by the bladder. Therefore, rather than wait 3-5 minutes to record data as in all other experiments in this chapter, data was recorded within 1 minute. However, the tests did corroborate the findings from "hard-to-soft" and "soft-to-hard' testing described elsewhere in this chapter, as can be seen in Table 30 and Table 31.

Table 30: Human testing based on hard cylinder calibration

\begin{tabular}{|c|c|c|c|c|}
\hline $\begin{array}{c}\text { Gauge pressure } \\
(\mathrm{mm} \mathrm{Hg})\end{array}$ & $\begin{array}{c}\text { Test 1 } \\
(\mathrm{mm} \mathrm{Hg})\end{array}$ & $\begin{array}{c}\text { Standard deviation } \\
(\mathrm{mm} \mathrm{Hg})\end{array}$ & $\begin{array}{c}\text { Test 2 } \\
(\mathrm{mm} \mathrm{Hg})\end{array}$ & $\begin{array}{c}\text { Standard deviation } \\
(\mathrm{mm} \mathrm{Hg})\end{array}$ \\
\hline 150 & 131 & 26 & 125 & 27 \\
\hline 200 & 192 & 36 & 182 & 42 \\
\hline 220 & 214 & 38 & 216 & 55 \\
\hline 240 & 236 & 40 & 244 & 60 \\
\hline 300 & 293 & 46 & 325 & 90 \\
\hline
\end{tabular}


Table 31: Human testing based on soft cylinder calibration

\begin{tabular}{|c|c|c|c|c|}
\hline $\begin{array}{c}\text { Gauge pressure } \\
(\mathrm{mm} \mathrm{Hg})\end{array}$ & $\begin{array}{c}\text { Test 1 } \\
(\mathrm{mm} \mathrm{Hg})\end{array}$ & $\begin{array}{c}\text { Standard deviation } \\
(\mathrm{mm} \mathrm{Hg})\end{array}$ & $\begin{array}{c}\text { Test 2 } \\
(\mathrm{mm} \mathrm{Hg})\end{array}$ & $\begin{array}{c}\text { Standard deviation } \\
(\mathrm{mm} \mathrm{Hg})\end{array}$ \\
\hline 150 & 192 & 50 & 185 & 37 \\
\hline 200 & 264 & 67 & 253 & 45 \\
\hline 220 & 294 & 71 & 283 & 49 \\
\hline 240 & 321 & 75 & 310 & 52 \\
\hline 300 & 396 & 79 & 386 & 60 \\
\hline
\end{tabular}

The measurements recorded when the sensor was calibrated on the hard cylinder are fairly close to the expected values. Considering that no time for equilibration was allowed, and that the standard deviations for the data are greater than fifty for almost all the recordings, these values are within the expected range. There are several possible explanations for the high standard deviations, but the most probable are that the sensor wrinkled, causing outliers in the data, or that part of the sensor was not completely under the bladder and therefore didn't experience enough pressure. It appears the human leg is between the soft foam and the hard plastic in hardness.

\subsubsection{Wrap on Human subject}

In order to test whether the system is working when the a human leg is wrapped with the neoprene bands, a TekScan sensor was positioned on a person's shin and thigh separately, and then both were wrapped. The anterior of the shin was the location of the sensor in Sim's experiments [14], as can be seen from the following figure. The exterior of the thigh is the location of the sensor in the experiments in this thesis, and therefore these locations allow for a direct comparison. The measurements were recorded immediately after the leg was wrapped and then again after two or three minutes had passed.

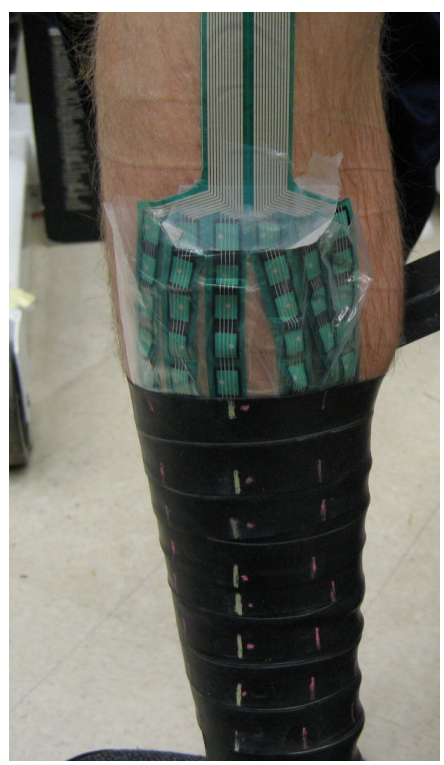

Figure 41: TekScan sensor wrinkling from the pull of the bands 
This process was done for calibrations on the hard cylinder, the soft cylinder, and the human leg. The human leg calibration was not performed according to the normal procedure because overpressurizing the human leg causes significant discomfort. The equilibrations were done in three different groups with the pressure released in between each. First, the sensor was equilibrated at 50, 100 , and $150 \mathrm{~mm} \mathrm{Hg}$. The second grouping was at 200 and $250 \mathrm{~mm} \mathrm{Hg}$. The last level done was $300 \mathrm{~mm} \mathrm{Hg}$. Furthermore, the calibration at $300 \mathrm{~mm} \mathrm{Hg}$ was only given one minute to equilibrate, instead of two, due to subject discomfort.

Since the human leg was the surface being measured, the human leg calibration was expected to be the most accurate, but the hard cylinder was calibration was closest to the expected value. This could be because the calibration procedure was changed or possibly the anterior of the thigh (used as the calibration surface as shown in Figure 40) does not have the same firmness as the exterior of the thigh or the anterior of the shin. If the neoprene bands produced the proper pressure, all the readings should have been $225 \mathrm{~mm} \mathrm{Hg}$.

Table 32: Wrapped measurements with different calibrations

\begin{tabular}{|c|c|c|c|c|c|c|}
\hline & $\begin{array}{c}\text { Soft } \\
\text { Calibration } \\
(\mathrm{mm} \mathrm{Hg})\end{array}$ & $\begin{array}{c}\text { Standard } \\
\text { deviation } \\
(\mathrm{mm} \mathrm{Hg})\end{array}$ & $\begin{array}{c}\text { Hard } \\
\text { Calibration } \\
(\mathrm{mm} \mathrm{Hg})\end{array}$ & $\begin{array}{c}\text { Standard } \\
\text { deviation } \\
(\mathrm{mm} \mathrm{Hg})\end{array}$ & $\begin{array}{c}\text { Thigh } \\
\text { Calibration } \\
(\mathrm{mm} \mathrm{Hg})\end{array}$ & $\begin{array}{c}\text { Standard } \\
\text { deviation } \\
(\mathrm{mm} \mathrm{Hg})\end{array}$ \\
\hline $\begin{array}{c}\text { Shin, } \\
\text { immediate }\end{array}$ & 273 & 74 & 205 & 75 & 163 & 56 \\
\hline $\begin{array}{c}\text { Shin, after } \\
\text { 3 min }\end{array}$ & 270 & 72 & 210 & 77 & 175 & 53 \\
\hline $\begin{array}{c}\text { Thigh, } \\
\text { immediate }\end{array}$ & 240 & 98 & 199 & 97 & 175 & 68 \\
\hline $\begin{array}{c}\text { Thigh, after } \\
\text { 2 min }\end{array}$ & 262 & 105 & 199 & 94 & 176 & 69 \\
\hline
\end{tabular}

It appears that for pressure measurements on the shin that the hard cylinder is the most appropriate choice for a calibration device, due to the hard, bony nature of the shin. For the thigh, which is a softer portion of the body, it is not immediately clear whether a soft or hard calibration is best. Given the softer nature of the thigh, the soft calibration will be used, although the results must be understood as somewhat inaccurate. The expectation was to have the human body between hard and soft calibration, but it appears that the human body was the softest. Part of the reason for this may be caused due to the short time periods used for calibration due to the discomfort it causes the subject. Calibration on a human, therefore, seems a poor idea, both due to the discomfort it causes, and the poor results evidenced in this table.

\subsection{Conclusions}

It is clear that the TekScan sensor is reading the accurate pressures when pressed against a particular surface only when it is calibrated on the same surface. "Soft-to-hard" testing leads to overly high measurements, since the sensor is expecting some surface deformation, but instead receives a strong force. In "hard-to-soft" testing, this hard surface is assumed but the deformation causes readings to be too low. These results indicate that the cause of the pressure variation is indeed with the TekScan system, not the manometer or the wrap. 
TekScan informs the user that the surfaces for interfacing with the sensor should be the same during calibration as for the testing. For that reason, a layer of plastic has always been kept on the sensor, as surface was interpreted to mean that physical layer with which the sensor was in direct contact. However, the team never understood this statement by the company to mean that if testing was taking place on a soft surface (ie skin), the calibration should take place on something soft as well (ie foam).

The most ideal calibration used for the thigh will be utilized for all the testing conducted in Chapter 6 , namely the soft cylinder. However, finding a more accurate pressure measurement system would be ideal. As mentioned in the introduction to this chapter, many alternatives were considered, of which the TekScan was chosen, although it was not the best option from a technical perspective. The Novel system is superior, but unfortunately is out of the budget range for this project [14]. Another option is a custom system being developed by the $16.62 \mathrm{x}$ students at MIT, although it was still in the conceptual design stage and unavailable for testing purposes at the time this thesis was written. 


\section{Chapter 5: Prototype refinement}

\subsection{Overview}

The entire set of hardware used to create mechanical counterpressure on the human leg will be outlined here in order to place all the necessary information to replicate these experiments in one place. As mentioned in the introductory chapter, this work builds upon the prototype developed by Liang Sim in his thesis, although some of his hardware has been replaced. In each section, it shall be made clear what updates, if any, have been made, so that Sim gets proper recognition for his work.

\subsection{Elastic bindings}

Sim in his thesis developed the elastic bindings ${ }^{20}$ concept in order to create MCP on the body. These elastic bindings are wrapped up the leg in a spiral fashion to the chosen position on the leg from the area above the ankle. Each layer of the band overlaps the previous layer, so that no skin is exposed to vacuum. It is important to note that this technique is a method for quickly and relatively easily producing MCP in the laboratory, and is not a proposed design for an actual spacesuit. After considerable testing of elastic materials for the purposes of producing of MCP, Sim selected black neoprene rubber, purchased from the Industrial Rubber and Plastic Company [14]. For this thesis, neoprene bands were purchased from this company as well, in lengths of 30 feet for a full leg wrap, with thicknesses of $1 / 32$ inch and widths of $1 \frac{1 / 2}{2}$ inches. These bands, while not an ideal material for this application, are able to meet the pressure requirements. Appendix I shows the Young's Modulus curve of the neoprene compared to the requirements developed in Chapter 2, as well as the entire calibration procedure. In addition to non-optimal stress-strain properties, the neoprene does not allow the user to sweat, which would require a significant thermal subsystem in the BioSuit. Additionally, neoprene is isotropic, whereas the ideal material would have a smaller Young's Modulus in the longitudinal direction than the circumferential direction to allow for greater flexibility and range of motion.

\subsubsection{Evolution of the wrap}

Sim used a single wrap in which one layer of material is used to create the MCP, with very little overlap of the bands as they are wrapped spirally up the leg, in experimentation. The wrap is "bottom-up" in that the first layer is just above the ankle, and then the wrapping spirals up until the seal (the design of which will be detailed in Section 5.4) is covered [14]. The bands must overlap, or else some skin will be exposed to significant underpressure, but this overlap creates small areas of overpressure on the skin.

\footnotetext{
${ }^{20}$ The elastic bindings are more commonly referred to in this thesis as the "neoprene wrap" or "neoprene bands." While the terminology is slightly different, all those phrases refer to the same material.
} 
Using this method to create pressure over the entire leg was quickly reconsidered however, when it became clear that the forces required by the test conductor to properly wrap the subject were too high over the upper thigh, as can be seen from Equation 1, since as the radius increases the amount of force needed to wrap the subject increases as well. For this reason, the team initially decided to use a single wrap up the leg until the knee and then double wrap the knee and thigh.

A double wrap is characterized by one layer of neoprene overlapping the top half of the previous layer (note the white lines down the length of the band for guiding the next layer in Figure 42).

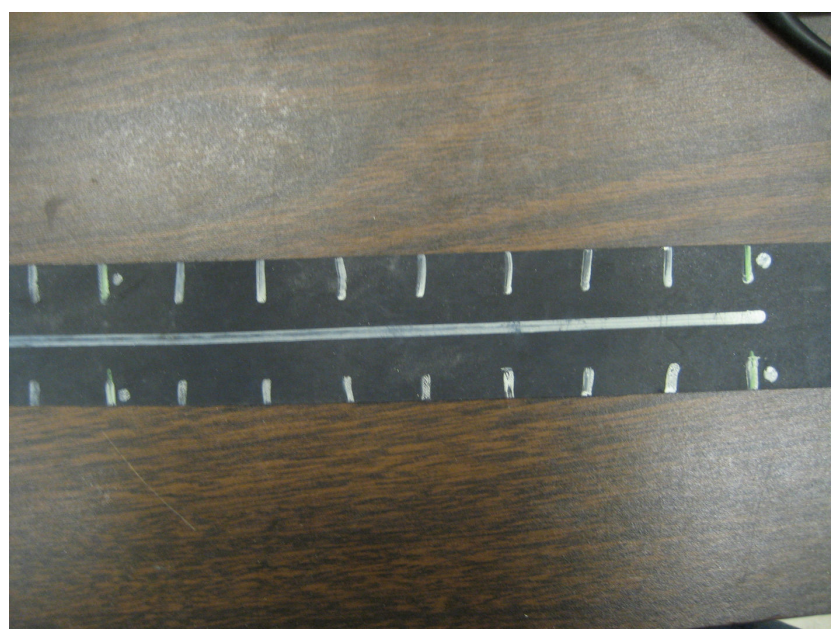

Figure 42: Portion of neoprene band

Each layer only has to produce $15 \mathrm{kPa}$, so the force required to stretch the wrap is cut in half, as again is evident from equation 1, making application of the bands much easier. The two layers combine to produce the $30 \mathrm{kPa}$ of pressure, as seen below.

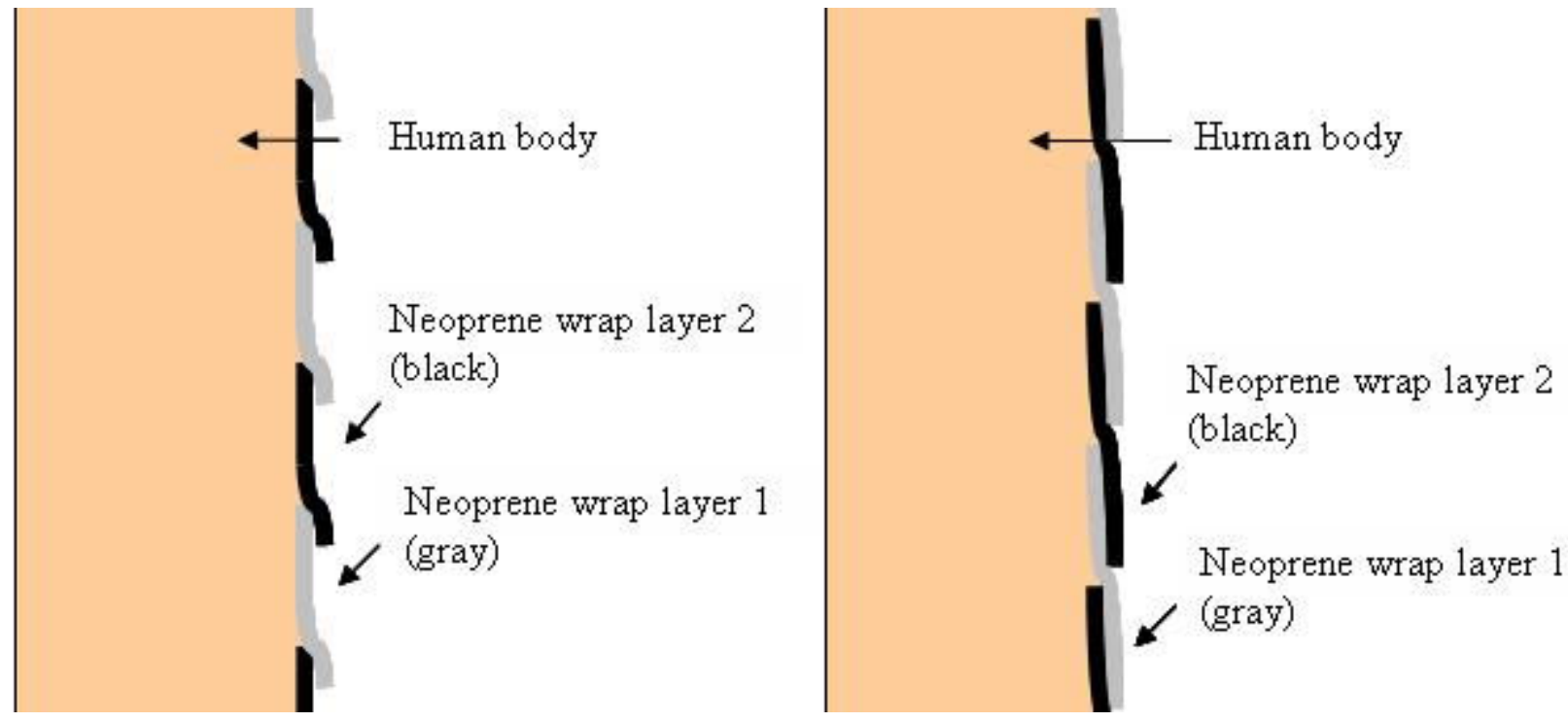

Figure 43: Visual explanation of single wrap and double wrap 
Note that in the figure on the left, there is very little overlap of the bands, with those areas that have multiple layers experiencing overpressure. On the right, the each layer of the wrap overlaps half of the previous layer which provides a more even pressure distribution with no points of overpressure. The layers of the neoprene wrap are colored separately to make the view easier, with one layer wrapped around the skin in gray, while the second is black. These bands would cover the entire leg (as seen in Figure 44), but this view is a cutaway to make the point more clearly.

However, this method of single wrapping to the knee, then double wrapping the knee and thigh, has 2 limitations: there are still bands of overpressure on the calf, but more importantly there is a narrow band of circumference across the knee that only experiences $15 \mathrm{kPa}$, which is sure to experience edema. Additionally, all the subjects noted that the double wrap was much more comfortable, and asked to have a double wrap over their entire leg.

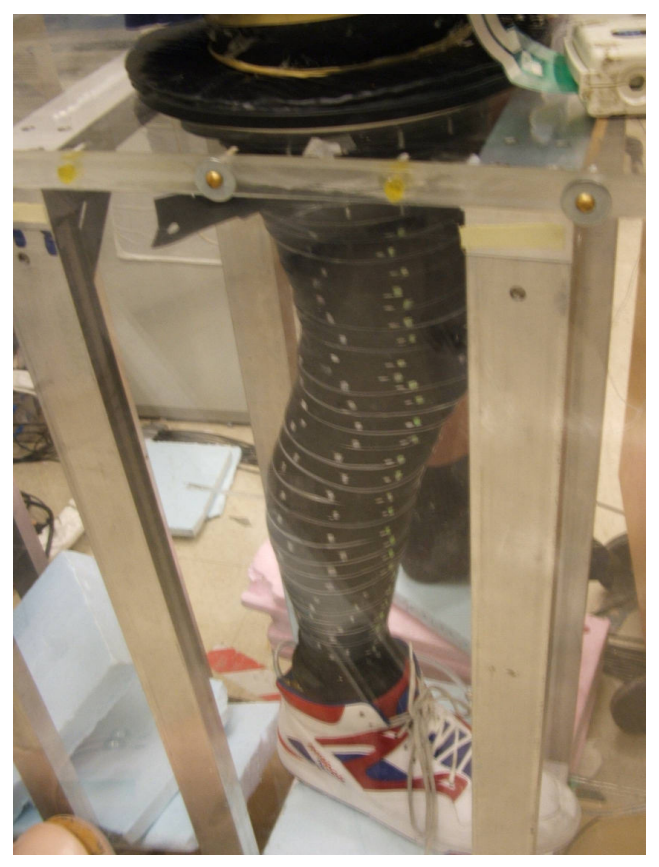

Figure 44: Subject wearing a double wrap in the chamber.

A few other ideas were tested to ensure that the double wrap is indeed the best solution. A triple wrap was attempted, but it significantly increased donning and doffing time, without any improvement in comfort or accuracy of pressure production compared to the double wrap. A topdown wrap, in which the test director begins the wrap at the top of the leg and then proceeds down the leg to the ankle was also attempted, but discarded after one test. Rather than tourniquet the foot and slowly squeeze the blood up into the torso as with a bottom-up wrap, this method tourniquets the leg, and then squeezes all of that blood into the foot, causing immediate discomfort, and, therefore, reduces the time available for donning, without any benefit. For these reasons, the bottom-up double wrap was chosen as the most accurate and comfortable wrapping procedure for 
the subject, despite the fact that it increases the donning and doffing time from a single wrap due to the additional layers of material. ${ }^{21}$

\subsubsection{Width selection}

Material of 0.75 inch and 1 inch width was purchased and then tested over both the knee and thigh of a human subject to see if a smaller width than the baseline 1.5 inch material could be used as it would be expected to provide more uniform pressure production. However, special care had to be taken (especially with the 0.75 inch wide material) during wrapping the leg in order to ensure that no bare skin was showing due to the smaller width. Even so, the material tended to shift, and small areas of skin pushed out from beneath the bands, and then became pinched by the neoprene in the process. The smaller width increased the donning and doffing time, as more spirals had to be wrapped to cover the entire leg. Therefore, the decision was made to stay with the 1.5 inch wide neoprene bands.

\subsubsection{Angle used for leg wrapping}

In order to wrap the entire leg, while still allowing mobility, a decision had to be made regarding the angle of the knee during material donning. The three angles tested were $0^{\circ}, 45^{\circ}$, and $90^{\circ}$ as they represent the 2 extreme values of typical human motion, with an average between them. The following observations were made for each of the angles:

\subsubsection{1 $0^{\circ}$}

At $0^{\circ}$, the wrapping of the leg is easiest, as the surface area on the back and front of the knee are approximately equivalent. Additionally, walking is relatively unconstrained, as during typical locomotion, the leg does not bend more than a few degrees.

However, bending to $90^{\circ}$ is difficult, as the neoprene resists contraction on the posterior of the knee, and creates bands of bare skin up to $1.5 \mathrm{~cm}$ in height on the patella. This bending also creates significant overpressure on the posterior of the knee.

\subsubsection{2 $\quad 45^{\circ}$ (approximate)}

For $45^{\circ}$, walking is relatively unconstrained as well, and there are smaller bands of bare skin (up to $0.5 \mathrm{~cm}$ on the patella) when the knee is bent $90^{\circ}$.

However, this angle is somewhat more difficult to wrap, since up to 4 bands are wrapped over the same piece of skin on the posterior of knee to completely cover the anterior of the knee, the patella. Additionally, because the patella extrudes farther from the other portions of the knee at this angle, the concavities around the patella experience no pressure in this configuration.

\footnotetext{
${ }^{21}$ A detailed procedure for the creation of each of these wraps is available in Appendix I.
} 


\subsubsection{3 $90^{\circ}$}

This method for wrapping creates full $30 \mathrm{kPa}$ of pressure on the anterior of the knee when bent $90^{\circ}$.

However, there is significant overpressure on the posterior of the knee at that same angle, since some cross-sections are covered by 5 layers of neoprene. It is difficult to walk in this configuration, as the many layers of neoprene make it difficult to straighten the leg.

\subsubsection{Conclusion}

The best method for actual movement would be to wrap at $45^{\circ}$ or some relatively neutral angle. However, donning will be done initially at $0^{\circ}$, which allows for the easiest application of MCP, as first uniform MCP must be produced on the leg before mobility can be tested.

\subsubsection{Measuring fatigue in the elastic wrap}

Since these bands were receiving a fair amount of use, both for measurements of leg circumference change (seen in Chapter 6) and actual testing (Chapter 6 as well), there was some concern that their material properties were changing, so some of the bands were retested to ensure that they were still able to produce the proper pressure. The following table shows an average of the changes in width and length of the bands tested when they were new compared to their properties after use in approximately 5 tests each.

Table 33: Comparison of tests between new and used bands

\begin{tabular}{|c|c|c|c|c|}
\hline & \multicolumn{2}{|c|}{ New bands } & \multicolumn{2}{c|}{ Used bands (5 tests average) } \\
\hline Applied force & Width & Length & Width & Length \\
\hline$(\mathrm{lbs})$ & $(\mathrm{mm})$ & $(\mathrm{mm})$ & $(\mathrm{mm})$ & $(\mathrm{mm})$ \\
\hline 0 & 38 & 100 & 38 & 100 \\
\hline 2 & 36 & 112 & 36 & 110 \\
\hline 4 & 35 & 127 & 34 & 132 \\
\hline 6 & 32 & 163 & 32 & 168 \\
\hline 8 & 30 & 205 & 30 & 201 \\
\hline 10 & 29 & 218 & 29 & 217 \\
\hline
\end{tabular}

An easier way to view the data is the following graph: 


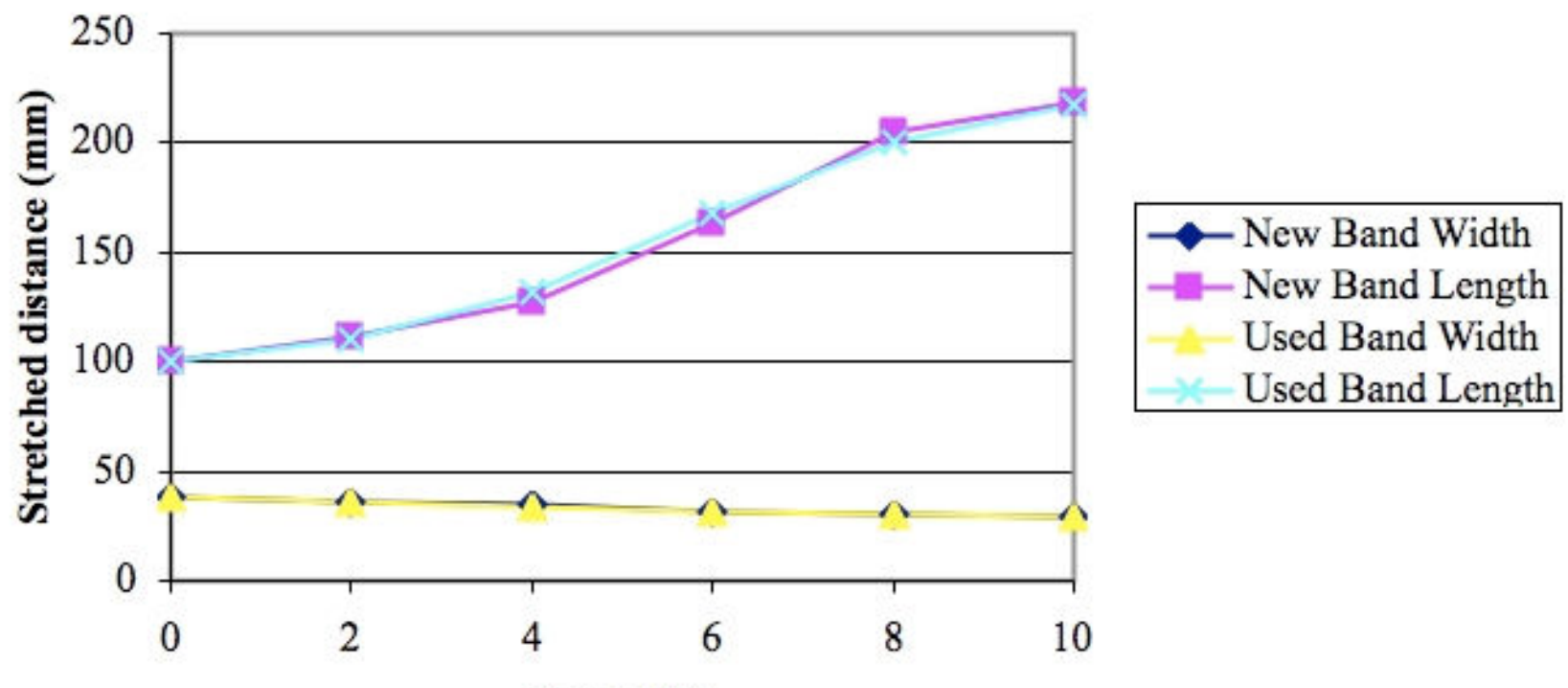

Force (lb)

Figure 45: Change in neoprene properties from new to used bands

There is some difference between the 2 values, but it is not significant as the band will continue to stretch under a constant force, making it difficult to accurately calculate the strain in the material, and therefore, introduces some inaccuracy in the measurements. However, with these results the bands can be used at minimum 5 times with confidence that they will produce the proper pressure.

\subsection{Pressure chamber}

The pressure chamber was developed by MIT undergraduate students, and is designed to fit a full human leg, as can be seen from the following figure. There is a 9 inch diameter hole in the top of the chamber for the subject to place his or her leg in the chamber, and the following section will detail the seal designs placed in this area to separate the atmosphere from the lower pressure inside the chamber (-30 kPa in the tests). 


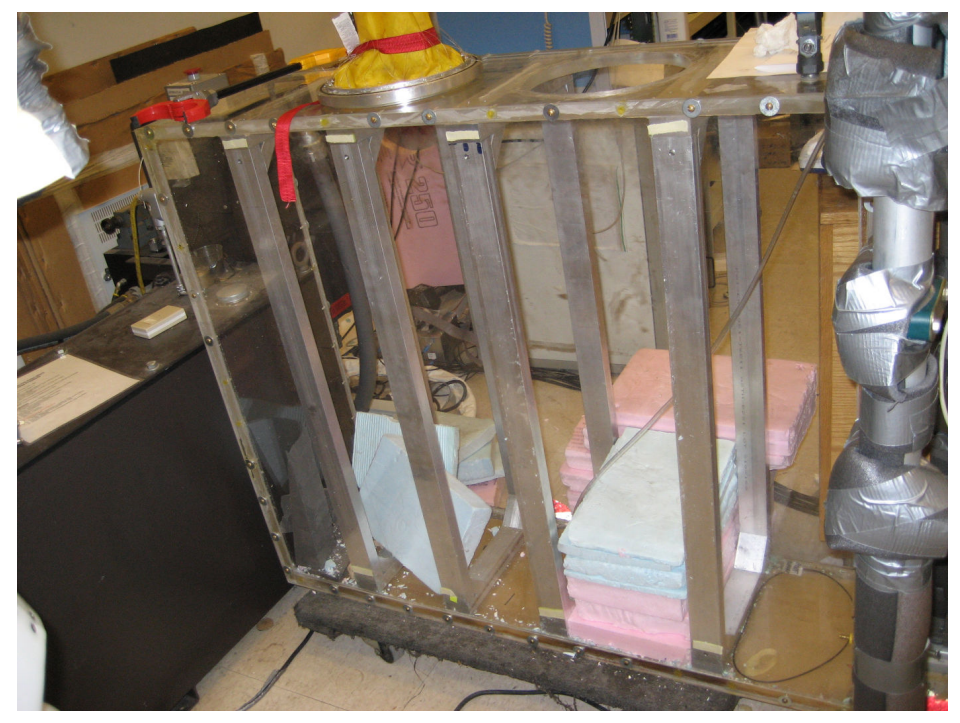

Figure 46: Low pressure chamber

\subsection{Seal between atmosphere and pressure chamber}

\subsubsection{Sim's work}

This piece of hardware proved to be the most difficult impediment to experimental testing. In Sim's experiment, two neoprene neck gaskets were sealed together with an adhesive, as shown in Figure 47, and then stretched over a metal ring with an o-ring that prevented air from leaking into the gap between the metal ring and the chamber, similar to the setup in Figure 49 on the right. The elastic bands were wrapped over the gaskets, thus stopping air from rushing into the chamber between the leg and the inner gasket. This design had some flaws however, as it failed in multiple ways. Air often would run between the leg and the seal into the test chamber if the subject moved, as there was a relatively small surface area that was compressing the skin. Once, the top of the seal was forced into the chamber by the difference in air pressure and expanded like a balloon, causing the test to be terminated. On another occasion, the adhesive connecting the two gaskets burst apart, allowing air to rush into the chamber [14]. Most importantly, however, the neck gaskets were too small to be placed over the upper thigh, as they were quite uncomfortable and a significant impediment to blood flow, so this design was not used for these experiments. 


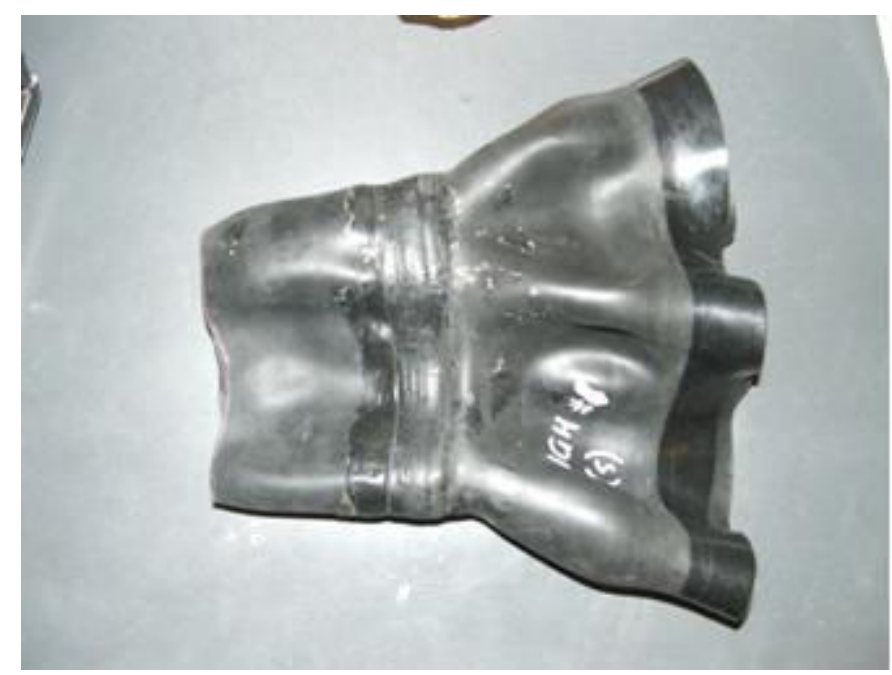

Figure 47: Initial seal design

\subsubsection{Second and third generation seals}

After considerable thought and testing, a slightly altered version of the original seal was chosen as a replacement. One neoprene rubber gasket was still used, but it was instead adhered to a neoprene knee sleeve, with a rubber band additionally adhered at the interface to strengthen this potential weak point. This design was superior to using 2 gaskets in several ways. First, it provided greater length for the wrap to compress on the skin, lessening the possibility that air would rush into the chamber if the subject moved his or her leg. Additionally, the design provided greater comfort for the subject, as the previous design acted somewhat like a tourniquet, severely constricting blood flow. This new design, while still tight on the skin, spread this pressure over a much larger surface area. The neoprene sleeve is much thicker than the gaskets, and provided an extra buffer zone between the metal ring and the skin, so there was less open area for the seal to be pushed into the chamber, partially mitigating the possibility of this failure occurring again. This design however, placed severe strain on the adhesive, and had to be fixed after every test. Figure 48 displays a subject wearing the second generation design, and Figure 49 shows that seal over the metal ring in the pressure chamber. 


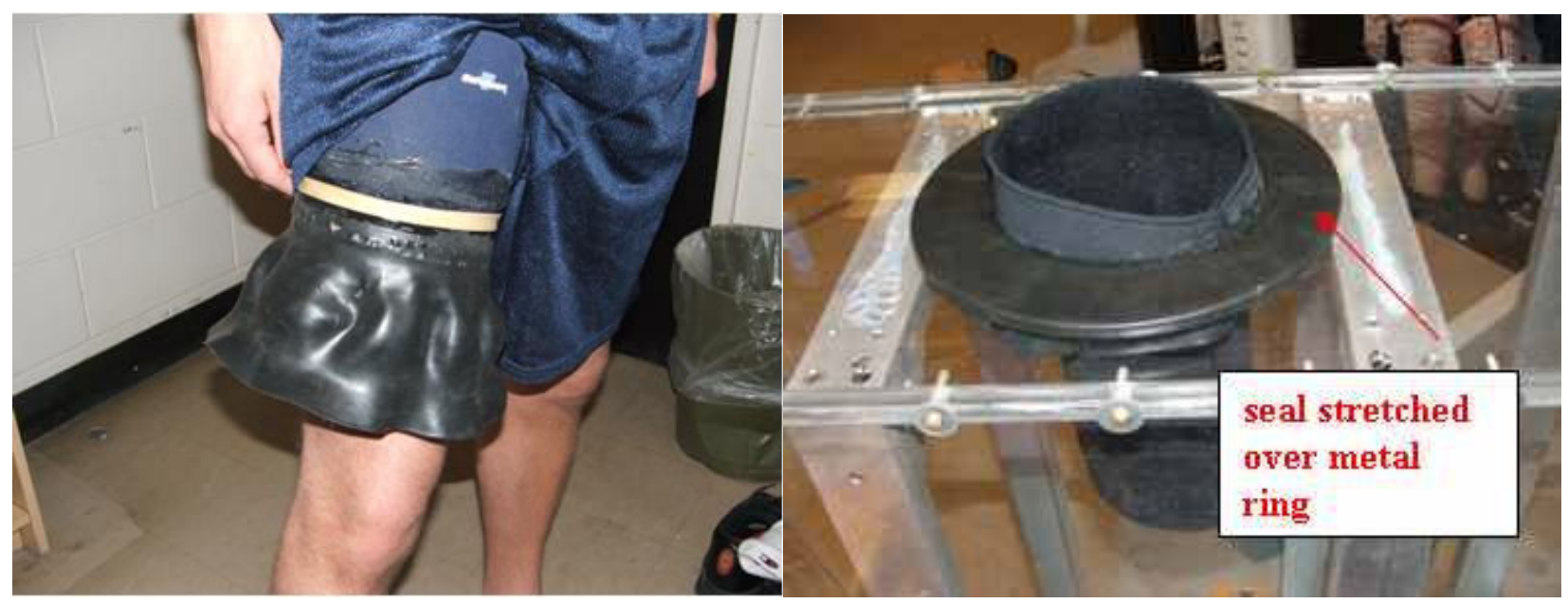

Figure 48 (left): Subject wearing second generation seal design

Figure 49 (right): Seal stretched over metal ring to separate pressure chamber from atmosphere around the leg

It was decided after further thought that a superior seal could be constructed, one that could better withstand the forces and require significantly less maintenance. A simple solution was found: adhere the seal upside down. With this new design (see Figure 50 below), a prototype seal was first created without actually using any adhesive, and tested successfully at a slight underpressure. The pressure would pull on both ends of the seal, meaning that if neoprene knee brace was tight enough against the skin, there would be no room for the air to rush into the chamber. However, to withstand the higher pressures, an adhesive was used to glue the gasket to the knee brace. Additionally, there was no need for a rubber band, which increased comfort, as previous subjects had complained slightly about lack of blood flow into their legs.

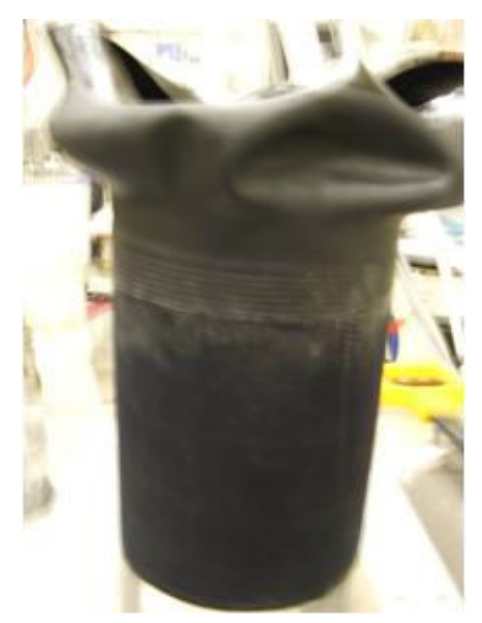

Figure 50: Third generation seal 


\subsubsection{ILC Dover seal design}

However, the third generation seal still proved inadequate, as the adhesive was not strong enough to handle the large pressure differential, and failed multiple times. Therefore a more robust design was needed, which preferably would be applicable to long-term testing, as a way to integrate MCP into a pressurized upper torso of a spacesuit for testing purposes in the future. Prof. Jeff Hoffman developed a concept in which an inextensible bladder material, composed of urethane coated nylon fabric is clamped between two metal rings, the bottom of which has an o-ring to create a seal from the atmosphere. ${ }^{22}$ The elastic bands are then wrapped over the bladder layer, so it provides a mechanism for ensuring that the proper $30 \mathrm{kPa}$ is being produced, since otherwise the force from the atmosphere will overcome the MCP and expand the bladder, causing the elastic bands to unwrap. More detailed information on the ring is available in Appendix $\mathrm{J}$.

On initial tests with this new seal, the difference in pressure between the chamber and the atmosphere provided sufficient force to roll the bands down the leg of the test subject, which created a tourniquet. The bands were providing the necessary pressure circumferentially, but are not designed to provide longitudinal pressure. Therefore, they were forced down the leg as can be seen in the following figure, creating bands of significant overpressure on the subject.
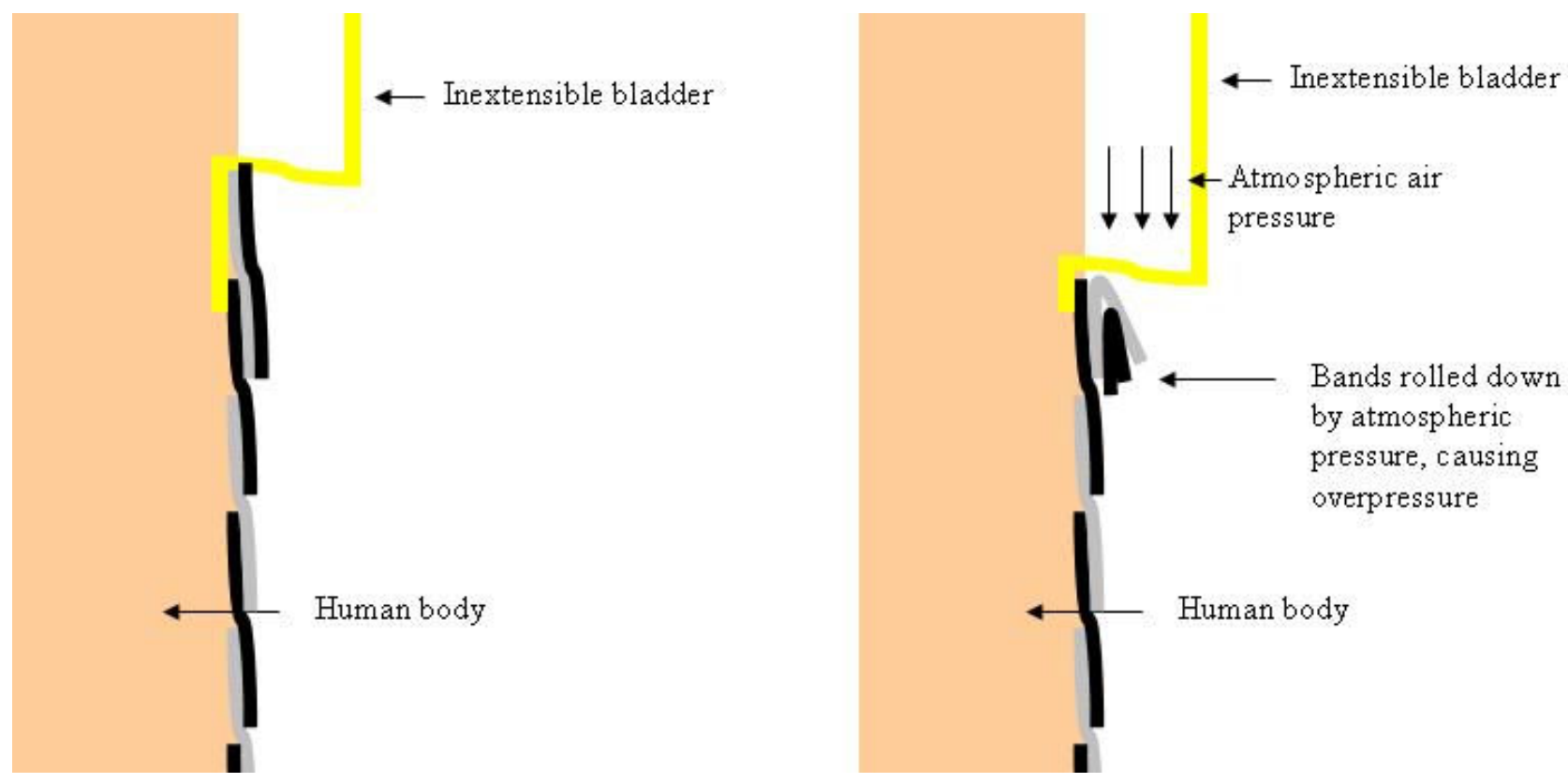

Figure 51: (left) Leg with new seal design

Figure 52: (right) Leg with new seal design and band rolling down the leg

A few ideas were then considered and tested to effectively handle the load occurring due to the pressure differential. The first option was to use strapping tape, an idea suggested by Prof. Hoffman. This design was partially successfully, as it was able to withstand a load of about 100

\footnotetext{
${ }^{22}$ Special thanks to Dave Graziosi of ILC Dover for providing 4 bladders free of charge, as all as CAD drawings of the 2 metal rings (although the bottom ring was redesigned to fit the hole in the pressure chamber).
} 
$\mathrm{mm} \mathrm{Hg}$, but still unable to prevent the band rolling down the leg. The next idea, also proposed by Prof. Hoffman, was to create a harness to handle the loads. A series of 24 holes for 4-40 screws were drilled in a small lip in the bottom metal ring (which is not drawn in the preceding figures for simplicity) and then a piece of fabric similar to a belt strap was hung from them by many pieces of fishing line (seen in Figure 54). This design experienced no major failures, but still had a fairly high leak rate, which is probably caused by the fact that the bladder diameter, while somewhat conical, is much larger than the subject's leg diameter. When neoprene bands are wrapped over the bladder, the bladder must be folded to stop air from leaking, although inevitably air leaks anyways. The following figures display the experimental seal design.

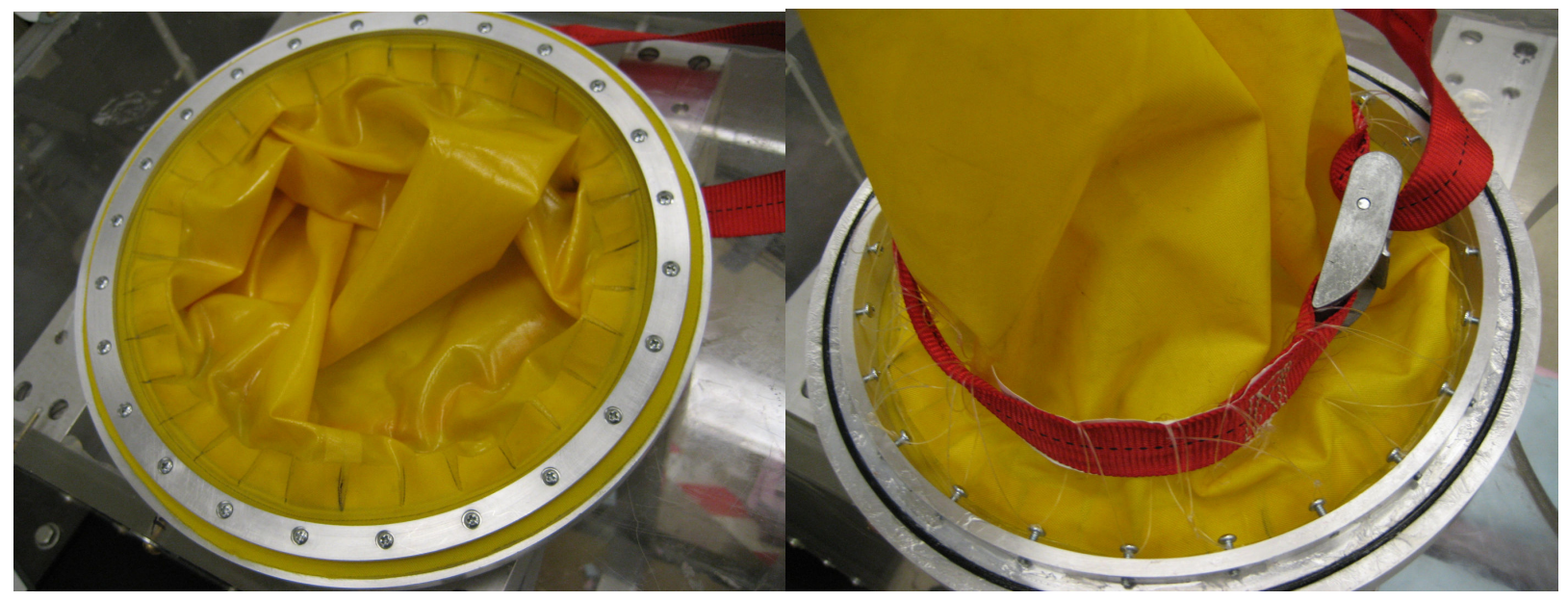

Figure 53: (left) Top view of seal

Figure 54: (right) Bottom view of seal

\subsection{Hybrid boot}

The elastic wrap only proceeds from the ankle to the seal, and does not protect the foot. The neoprene wrap can be used over roughly cylindrical surfaces, such as the human leg, but is harder to apply to the non-uniform geometry of the foot. As the purpose of this work is to identify MCP as a viable design concept, there was no rationale for attempting to apply MCP to the foot, which is a more difficult problem. Therefore, another method besides MCP was found to pressurize the foot. Sim, after significant testing, developed a hybrid design which consists of 2 XL Sealskinz Water Blocker socks sealed together using Marine Goop (Eclectic Products, Lineville, LA) to create a bladder. These socks are placed over the foot, and a small bulkhead fitting (Part no. 5454K85, McMaster-Carr, New Brunswick, NJ) is attached to the outer sock below the blue line. The space between the socks acts as a bladder and ensures that the foot is pressurized at atmospheric pressure through an air tube that is connected to the outer atmosphere. A large basketball shoe (size 15) is used as a restraining layer, so that the socks expand to an acceptable distance, and do not place too much stress on the adhesive. No changes were made to this design developed by Sim, and a detailed procedure for making the socks is available in Appendix K [14]. 


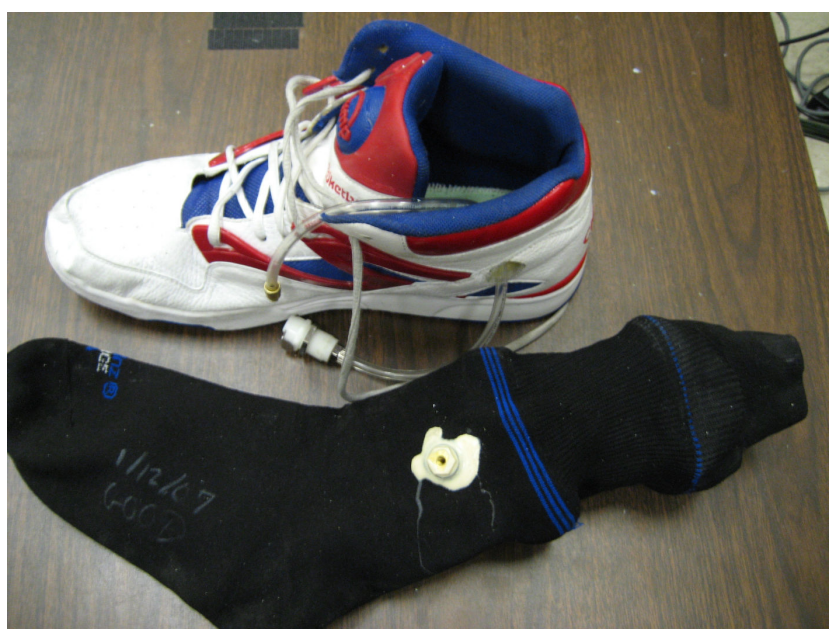

Figure 55: Hybrid sock and restraining shoe 


\section{Chapter 6: Experimental testing}

\subsection{Motivation}

As discussed in Chapter 2, there are two studies that have included testing of human legs: the Space Activity Suit developed by Webb and Annis, and the elastic bands prototype developed at MIT. However, these studies each have limitations: the testing time of the SAS was limited at full underpressure (no more than 20 minutes) while the previous tests at MIT outlined in the Sim thesis were only conducted on the calf. While the bands successfully protected this portion of the body, it remains unclear whether MCP could successfully pressurize a major joint, such as the knee or elbow. The concavities in those regions of the body may not be easily pressurized, so one goal of this thesis was to conduct tests of at least an hour duration over the full leg so that the ability of MCP to produce the proper pressure over the knee and the thigh could be more fully investigated.

\subsection{Experimental procedure}

The experimental procedure, which was approved by the MIT Committee on the Use of Humans as Experimental Subjects (COUHES), consisted of a 6 minute time period for baseline measurements followed by an hour test in the pressure chamber at $-225 \mathrm{~mm} \mathrm{Hg}$ (relative to atmospheric pressure). A group of 3 men and 4 women participated in the pilot study and main study.

The subject first stood outside the chamber for a period of 6 minutes to provide baseline data with only the equipment listed below on their leg. The following measurements were taken:

- heart rate

○ Acumen TZ-Max100 heart rate monitor

- blood pressure

O Omron HEM-711 blood pressure cuff with LoggerLite software

- skin temperature in 4 places (anterior of right shin, posterior and anterior of right thigh, and the anterior of the left thigh)

- Pasco PS-2135 Fast Response Temperature probes placed on the skin with Pasco PS2525 temperature adhesive patches

After completing the baseline measurements, the test director began to wrap the subject with the elastic bands and the additional assorted MCP materials in the following manner ${ }^{23}$ :

1. The TekScan sensor was placed on the exterior of the subject's right leg, with the bottom portion around the knee, depending on the thigh length of the subject.

2. The seal (either the neoprene sleeve or the inextensible bladder) was placed over the TekScan sensor on the upper thigh.

3. The elastic bindings were wrapped over the subject's leg from just above the ankle to the top of the seal.

\footnotetext{
${ }^{23}$ A more detailed breakdown of the experimental procedure is available in Appendix L. This section is only meant to give the reader a general overview of the procedure.
} 
4. The test director or an assistant placed the hybrid sock, and subsequently the shoe on the subject.

5. The subject carefully entered the chamber, taking care not to bend his/her knee, else the bands would slip.

6. Once the subject was in the chamber and the seal was securely placed, the pressure pumps were activated until the pressure in the chamber reached a differential pressure of $-225 \mathrm{~mm}$ $\mathrm{Hg}$ (with respect to normal atmospheric pressure).

7. The data recording and timing began immediately when the chamber first reached $-225 \mathrm{~mm}$ $\mathrm{Hg}$.

The measurements mentioned previously in the baseline measurements (heart rate, blood pressure, skin temperature) were all taken while the subject was in the chamber, as well as TekScan pressure measurements. Additionally, a qualitative metric system was devised for subject comfort levels. The scale was explained to the subjects as follows:

Table 34: Subject comfort subjective scale

\begin{tabular}{|c|l|}
\hline Scale Value & \multicolumn{1}{c|}{ Explanation } \\
\hline 0 & Normal sensation \\
\hline 1 & Somewhat abnormal sensations, but no discomfort \\
\hline 2 & Mild, but tolerable discomfort \\
\hline 3 & Some unpleasant sensations, increased discomfort \\
\hline 4 & Significant discomfort \\
\hline 5 & Too much discomfort to continue \\
\hline
\end{tabular}

The comfort ratings, along with the blood pressure measurements, were recorded every 2 minutes, while the heart rate and skin temperature were recorded every 5 seconds. The TekScan pressure data was recorded every 5 minutes.

\subsection{Pilot study}

The initial intention of this thesis was to only conduct one set of experiments. However, after completing 4 tests, it became quite evident that the neoprene seal design was inadequate, as was discussed in the previous chapter. One of the tests outlined here was ended due to a seal failure, and a handful of others were terminated within the first 5 minutes for the same reason. After each of the tests, the seal needed to be repaired as well. For these reasons, testing stopped while a new design was developed. However, the results of this pilot study influenced the main study developed in the following section, and should be discussed as some of the findings were quite important.

Since the main study contains much more data, these experimental results will be discussed mostly at a qualitative level.

\subsubsection{Pilot study results}

Subject 1 in this study, a 26 year old woman who is $175 \mathrm{~cm}$ tall and weighs $70 \mathrm{~kg}$, ended the experiment after 32 minutes due to discomfort. She also experienced some edema, as seen in Figure 
56, with the oval highlighting the most significant swelling. Subject 2 (62 year old man, $188 \mathrm{~cm}, 78$ $\mathrm{kg}$ ) was forced to terminate the experiment due to a seal failure at 36 minutes, but had a very low discomfort level, 2 out of a maximum of 5 . Figure 57 shows his legs, including the minor edema on the right leg, centered around the knee, of Subject 2 after the experiment. In both figures, the circumferential marks, which typically lasted a few hours, caused by the edge of the neoprene bands can be seen.

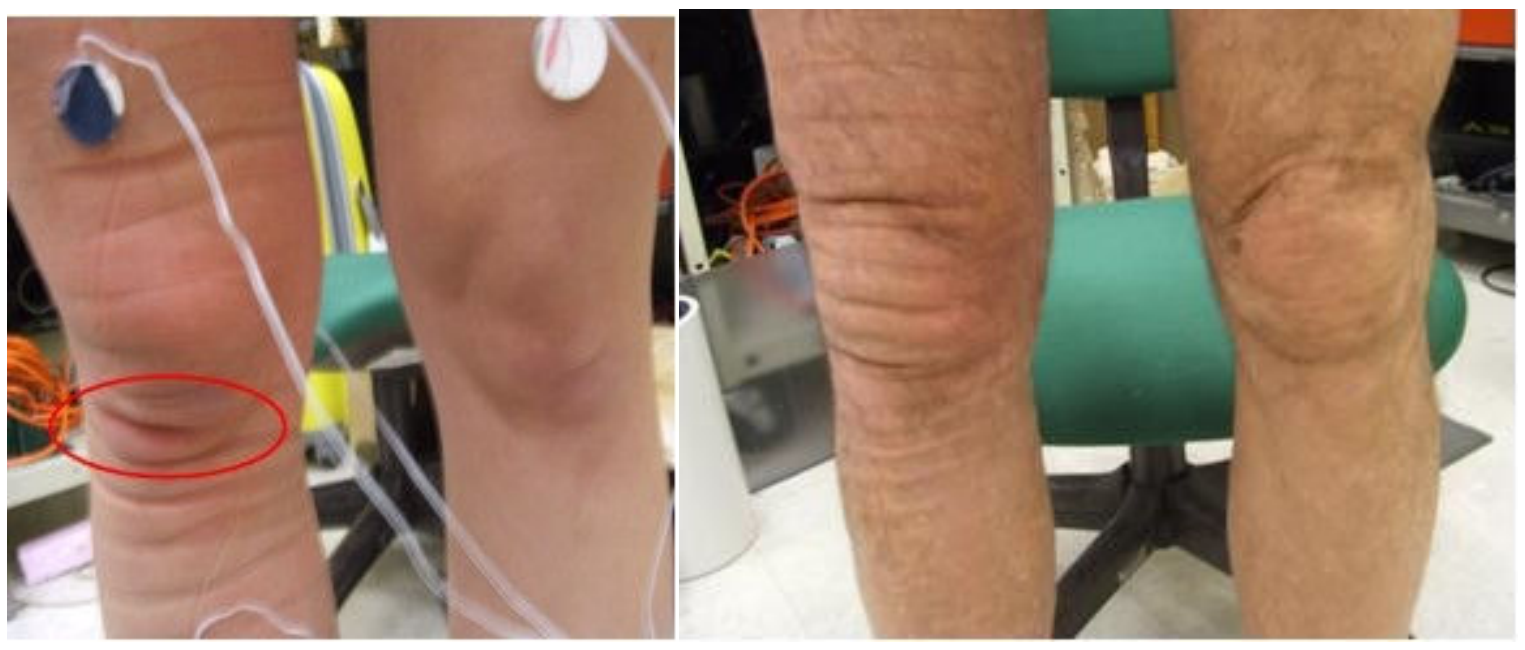

Figure 56: (left) Subject 1 after $32 \mathrm{~min}$ in the pressure chamber

Figure 57: (right) Subject 2 after $36 \mathrm{~min}$ in the pressure chamber

Neither subject experienced significant changes in heart rate, blood pressure, or skin temperature, other than a $2{ }^{\circ} \mathrm{C}$ increase in the skin temperature on the anterior of the right leg of Subject 1, which was in the chamber.

Subject 3, (28 year old male, $180 \mathrm{~cm}, 79 \mathrm{~kg}$ ) similarly experienced no significant physiological changes, even though this subject had skin exposed to the $-225 \mathrm{~mm} \mathrm{Hg}$ underpressure for the last 15 minutes of the test (as seen in the following figure, with the exposed skin highlighted in the oval). The test director takes great care to cover the entirety of the leg of every subject who enters the pressure chamber, but the bands sometimes slide when the subject climbs into the chamber, even though significant effort is made to ensure that the knee of the subject is not bent upon entry into the chamber. In this case, the bands must have slid apart during the test, until they separated completely. Despite this significant local underpressure, Subject 3 was only a 4 on the discomfort scale (significant, but tolerable discomfort). 


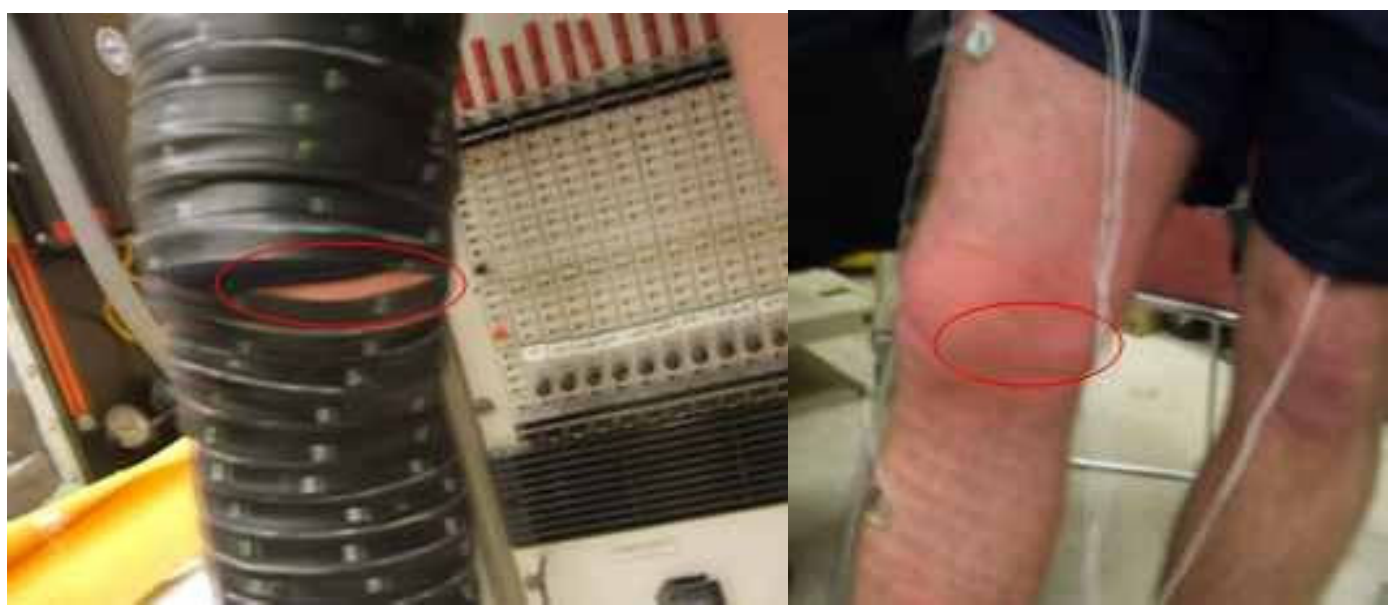

Figure 58: (left) Skin showing inside the leg wrap in the chamber.

Figure 59: (right) Subject's leg after exiting chamber

The subject did experience edema (highlighted in the oval in Figure 59), but it had all disappeared within approximately 8 hours. It is interesting that Subject 3 showed no increase in skin temperature, which often correlates with edema. The experiment was ended after 38 minutes by the test director, who was concerned for his health, but Subject 3 still was willing to stay in the chamber longer.

Subject 4 (26 year old woman, $163 \mathrm{~cm}$ tall and $59 \mathrm{~kg}$ ) was unable to complete the experiment, which was ended after 13 minutes, because she experienced a physiological reaction to underpressure. The most useful data gained from this test was her skin temperature, which will be presented here. A more thorough investigation of her test data is available in Appendix M.

The sensor on the left leg outside the chamber (Temp. 4) shows very little movement throughout the time period, while sensor 1 (shin anterior) and sensor 2 (thigh posterior) show a gradual rise. Part of the reason for this rise is that a certain amount of time (kept as short as possible, but usually a few minutes) elapses between completing the pressure-wrapping of the leg and the subject entering the chamber. During this time, the leg experiences $\sim 30 \mathrm{kPa}$ overpressure, which impedes circulation. Once the leg is in the chamber and the chamber is pumped down, blood flow increases, and the temperature rises. The most interesting result is the rather large $\left(3.4{ }^{\circ} \mathrm{C}\right)$ rise in sensor 3 (thigh anterior). All of these trends can be seen in the figure below (please note, however, that the scale on the y-axis begins at $28^{\circ} \mathrm{C}$ ). 


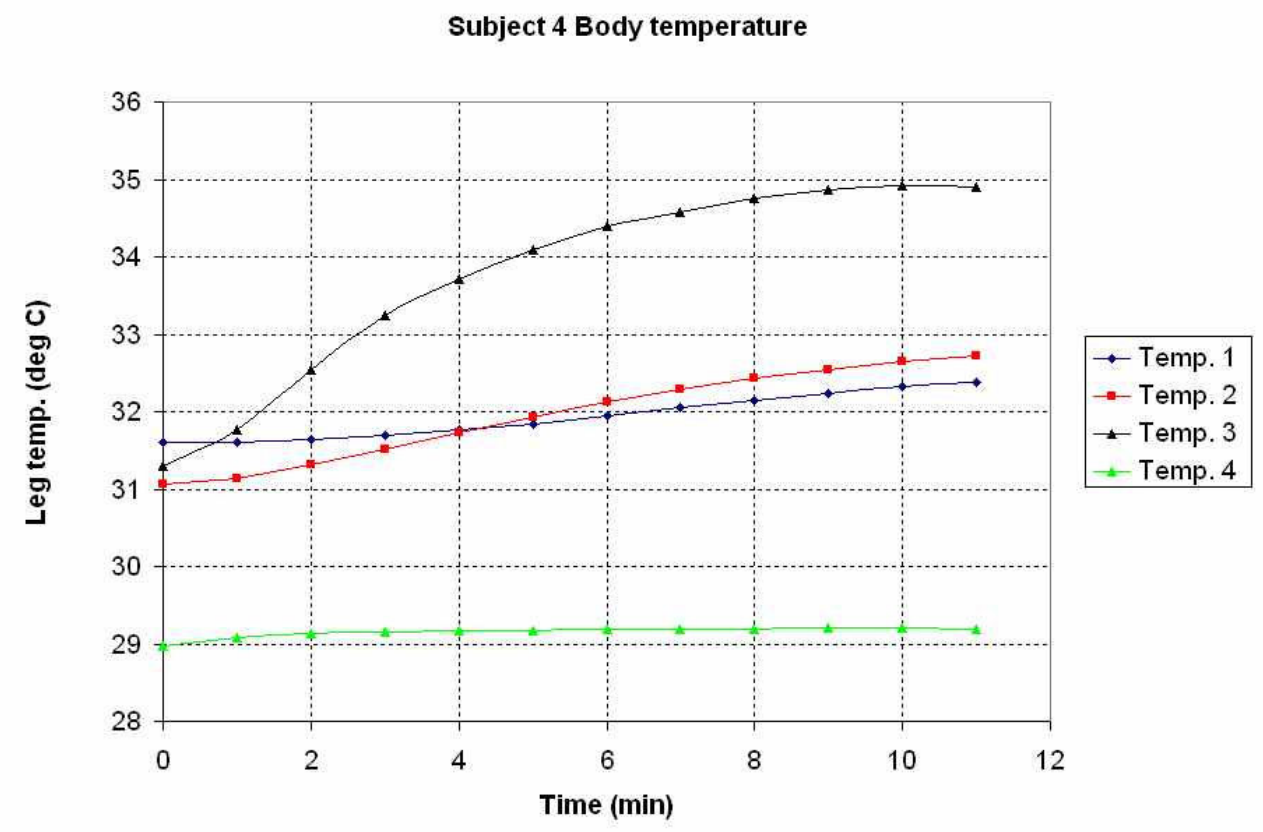

Figure 60: Temperature sensor readings on Subject 4

No other test subjects showed such a large temperature change. Subjects 2 and 3 showed no temperature change much larger than $1{ }^{\circ} \mathrm{C}$. Subject 1 showed approximately a $2{ }^{\circ} \mathrm{C}$ increase on the thigh anterior, but otherwise had the small $1{ }^{\circ} \mathrm{C}$ changes as well. It is important to note that Subject 1 ended the experiment for discomfort reasons, while Subjects 2 and 3 ended due to a mechanical failure and at the test director's prerogative, respectively.

\subsubsection{Pilot Study Discussion}

The pilot study produced a number of interesting results, which helped improve the experimental procedure and MCP bands. The first point is that Subject 3 was able to comfortably tolerate a small band of skin exposed to significant undepressure. This subject's experience gives credence to the theory that even if a MCP spacesuit rips, and some skin is exposed, the astronaut would have some time to find a pressurized shelter or patch the tear before significant physiological damage would occur while on the Moon or Mars. Additionally, even if such damage were to occur on another planetary surface, it would be local and temporary, whereas if the subject was wearing a gaspressurized spacesuit, life-preserving oxygen would be leaking into space.

The second major point was the investigation of the underpressure experienced by Subject 4 . Ideally, the TekScan sensors would be able to sense the underpressure, but throughout the experimentation process they have been unreliable, as was explained in Chapter 4. It was decided that a potential cause of this underpressure was the calibration of the neoprene wraps for the individual wearer, which was based on the cross-sections of the subject's leg measured under normal atmospheric pressure. The elastic bands are wrapped around each cross-section with enough stretch to produce a circumferential force which in turn produces the desired pressure on the 
leg. However, as the leg compresses under pressure and the circumference decreases, the elastic wrapping is not stretched to the appropriate strain, which in turn reduces the pressure on the skin. The following figures display the changes in circumference for two subjects (Subject 1 and 4) ${ }^{24}$ from atmospheric pressure and then with the neoprene wrap.

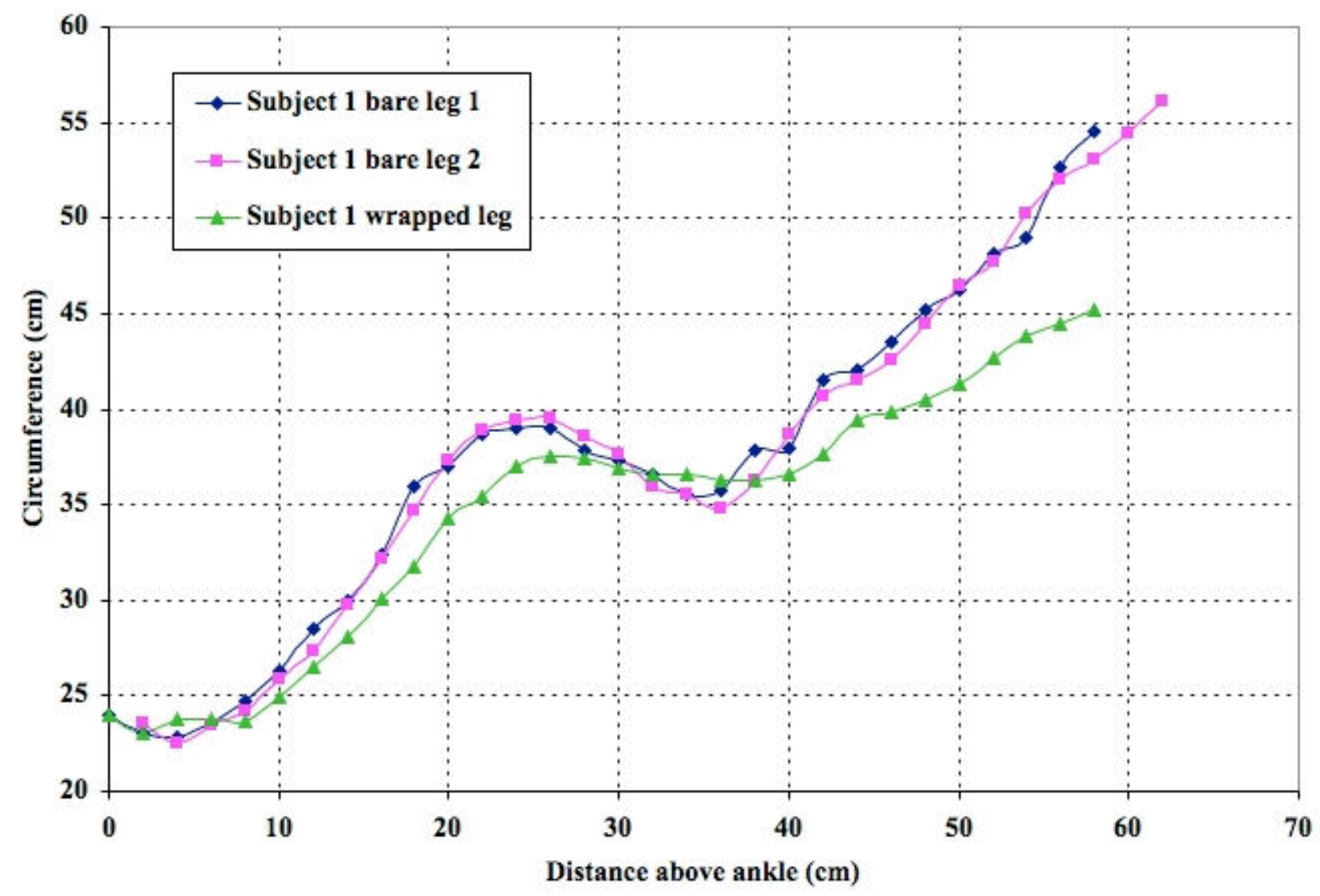

Figure 61: Change in circumference for Subject 1 between bare leg and leg experiencing MCP

The center of the patella of Subject 1 is located at approximately $38 \mathrm{~cm}$. Note that her upper calf compressed somewhat, although a much more significant deformation can be seen on her upper thigh. The compression on Subject 4's leg is not quite as large, but still enough to cause the bands not to properly pressurize her leg. The center of the patella of Subject 4 is similarly located at approximately $38 \mathrm{~cm}$.

\footnotetext{
${ }^{24}$ The measurements taken from the subject's leg under MCP outside the chamber were taken before her experiment. After her reaction to underpressure she was excused from further testing.
} 


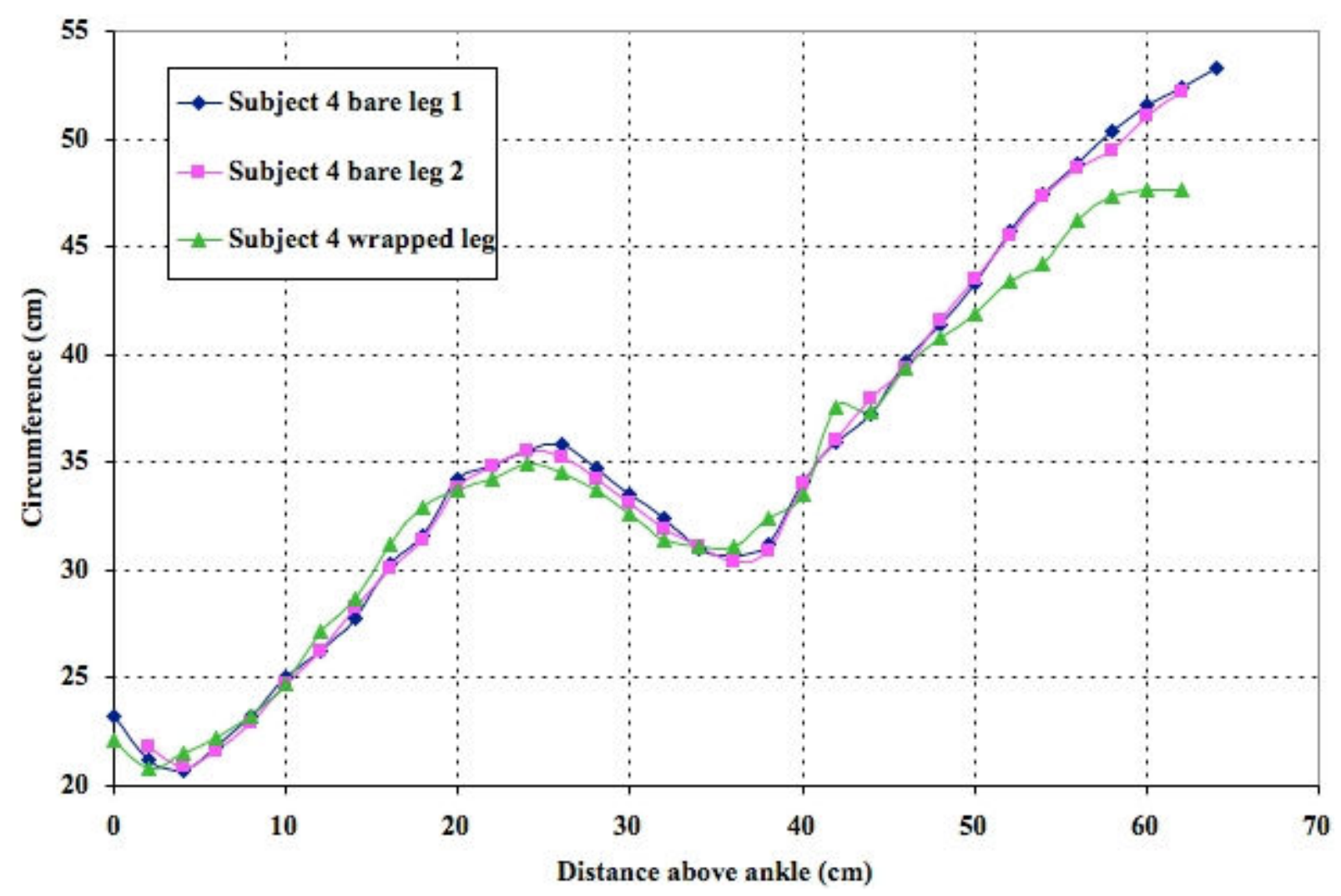

Figure 62: Change in circumference for Subject 4 between bare leg and leg experiencing MCP

Given the change of circumferences measured with and without pressure-wrapping, the team was able to calculate what the actual pressure production on the leg would for a "perfect" wrap, ("perfect" with relation to the normal, unwrapped leg). The results can be seen in the following table and Appendix N contains the Matlab code used to calculate these values.

Table 35: Calculated pressure for Subject 4

\begin{tabular}{|c|c|}
\hline Distance above ankle $(\mathrm{cm})$ & $\begin{array}{c}\text { Actual Pressure } \\
(\mathrm{kPa})\end{array}$ \\
\hline 10 & 31.68 \\
\hline 20 & 29.53 \\
\hline 30 & 29.63 \\
\hline 40 & 29.54 \\
\hline 50 & 27.96 \\
\hline 60 & 26.80 \\
\hline
\end{tabular}

The highlighted cells show the portions of the thigh which were experiencing more than $1.6 \mathrm{kPa}$ of underpressure. A similar analysis was conducted on the other subjects, and Subject 1 also must 
have been experiencing significant underpressure on her upper leg, which explains the large discomfort variation between the 2 women (Subjects 1 and 4) and the 2 men (Subjects 2 and 3). It seems likely that the major contributing factor to this difference is the variation on the fat content in the leg. Women have a larger amount of subcutaneous fat than men, which is far more compressible than muscle or bone. Therefore, greater care must be taken, especially for female subjects, to ensure the elastic bands are producing the appropriate pressure.

\subsubsection{Pilot study changes}

A number of changes were made to the study after the results of the pilot experiments were analyzed, and they are detailed below.

1. The calibration of the wrap became an iterative process, and measurements of the subject's wrapped leg were taken to see how much compression the leg experiences when pressurewrapped. The compressed leg circumference were used to calculate the final stretch required in the elastic strap. With this new data set, new bands take into account this change when they are calibrated.

2. The temperature sensors were watched carefully for any large increases in skin temperature in the subjects in the main study.

3. The seal was redesigned in order to increase reliability, as discussed in the previous chapter.

\subsection{Main study}

The main study for this thesis consisted of 5 subjects: 2 men and 3 women. Two of the subjects from the pilot study (Subjects 1 and 2) participated in this main study as well. Before the data is presented, a brief summary of the experiences of the subjects shall be stated, so that the data can be more clearly interpreted.

Subject 1 experienced some edema, although less than in her previous test. The edema was most noticeable below her kneecap, and she had some general redness around her knee. During the test, two layers of the neoprene wrap separated, exposing some skin to the $-225 \mathrm{~mm} \mathrm{Hg}$ underpressure in the chamber. However, like Subject 3 in the pilot study, she was able to continue the experiment and complete the full hour of nominal testing. The test conductor followed up with the subject after the examination, and she revealed that an hour later much of the swelling had subsided and the marks caused by the double wrap were significantly less noticeable as well. Figure 63 displays the leg of the subject immediately after exiting the chamber and Figure 64 shows her leg an hour later. 


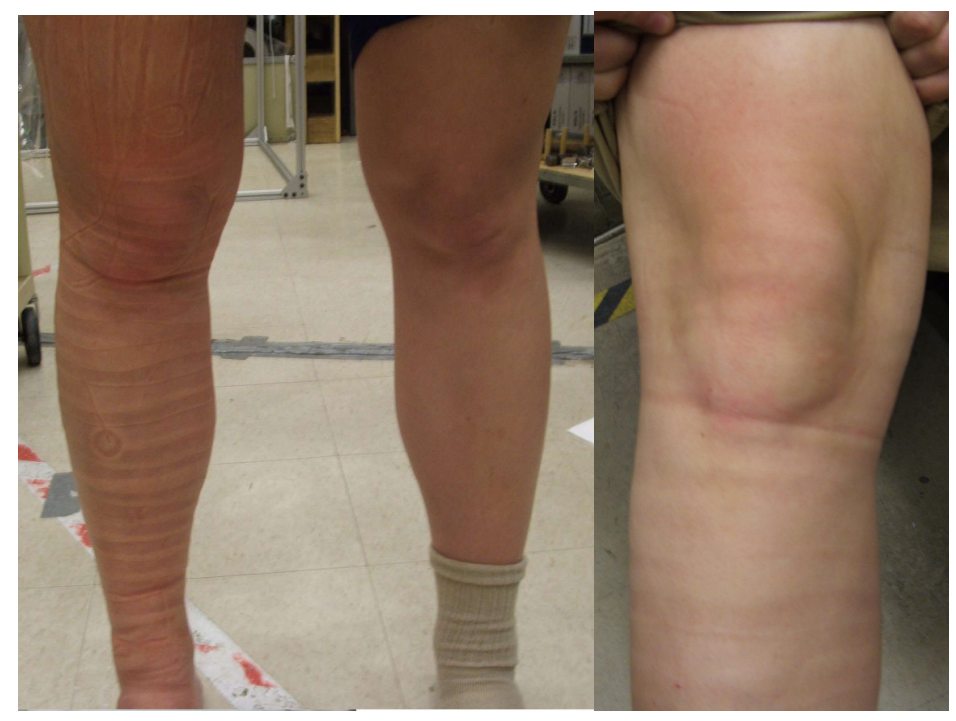

Figure 63: (left) Subject 1 immediately after exiting the chamber

Figure 64: (right) Leg of Subject 1 an hour later

Subject 2 completed the main study experiment two separate times. In the first test, the subject experienced some edema on his knee, as shown in the following figures. This subject also had two overlapping bands slide apart in his double wrap as well, exposing his skin to the underpressure of the chamber. He experienced some edema in this area (in the oval in Figure 66), but again, it was relatively minor.

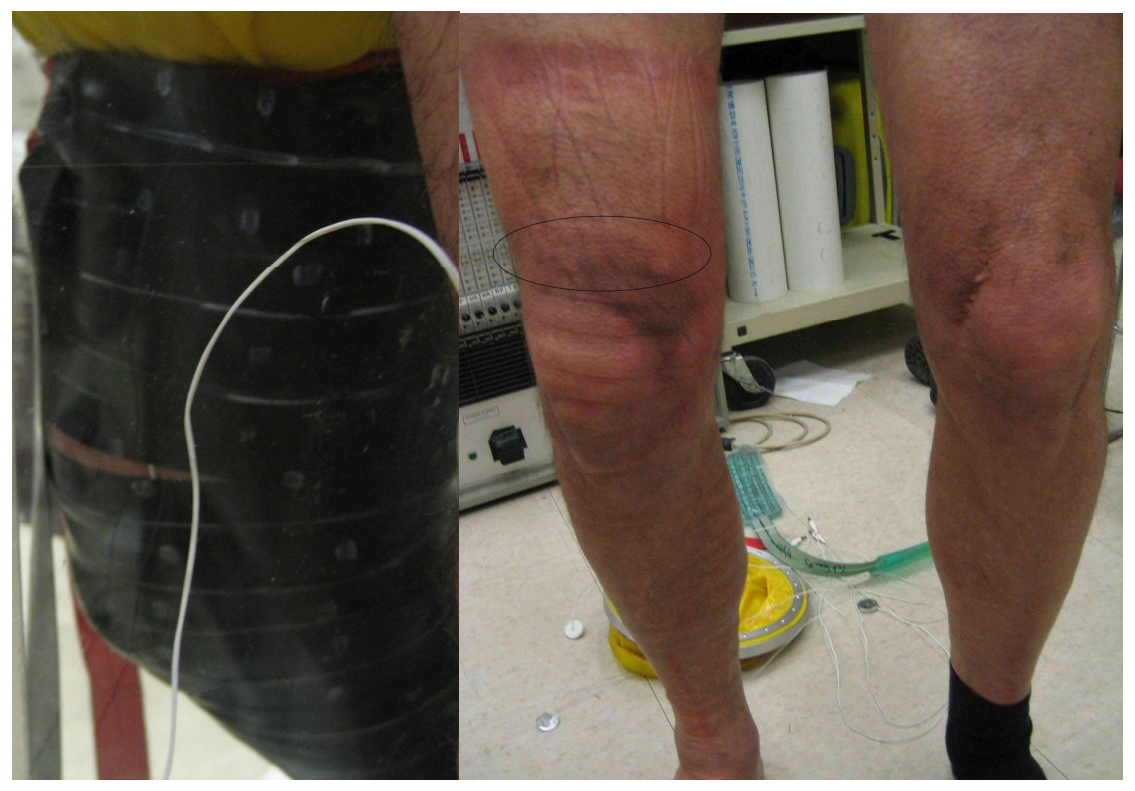

Figure 65: (left) Leg of Subject 2 in the chamber, with skin exposed to underpressure Figure 66: (right) Legs of Subject 2 after the experiment 
For the second test the subject wore a Champion knee brace with Hor-Shu support pad, as well as a small piece of foam under the knee cap, as seen in Figure 67. The reason for wearing the knee brace was to test whether it would stop edema from occurring on the knee, as the experiences of the subjects in the pilot study as well as the main study indicate that the neoprene wrap is not effectively pressurizing the concavities around the knee. The knee brace was meant to "circularize" the cross-sections around the knee by placing material in the concavities of the knee, so that the neoprene bands could be more effective. After completing the full hour, the subject had very little edema, much less than any of the other subjects, and found that the edema that did occur had almost completely subsided within 30 minutes. For this test, the same neoprene band was used as in the previous experiment, so the added bulk of the knee brace was not taken into account when calibrating the markings to produce pressure. Therefore, the bands should have provided more than the desired $225 \mathrm{~mm} \mathrm{Hg}$ of pressure to the area around the knee.

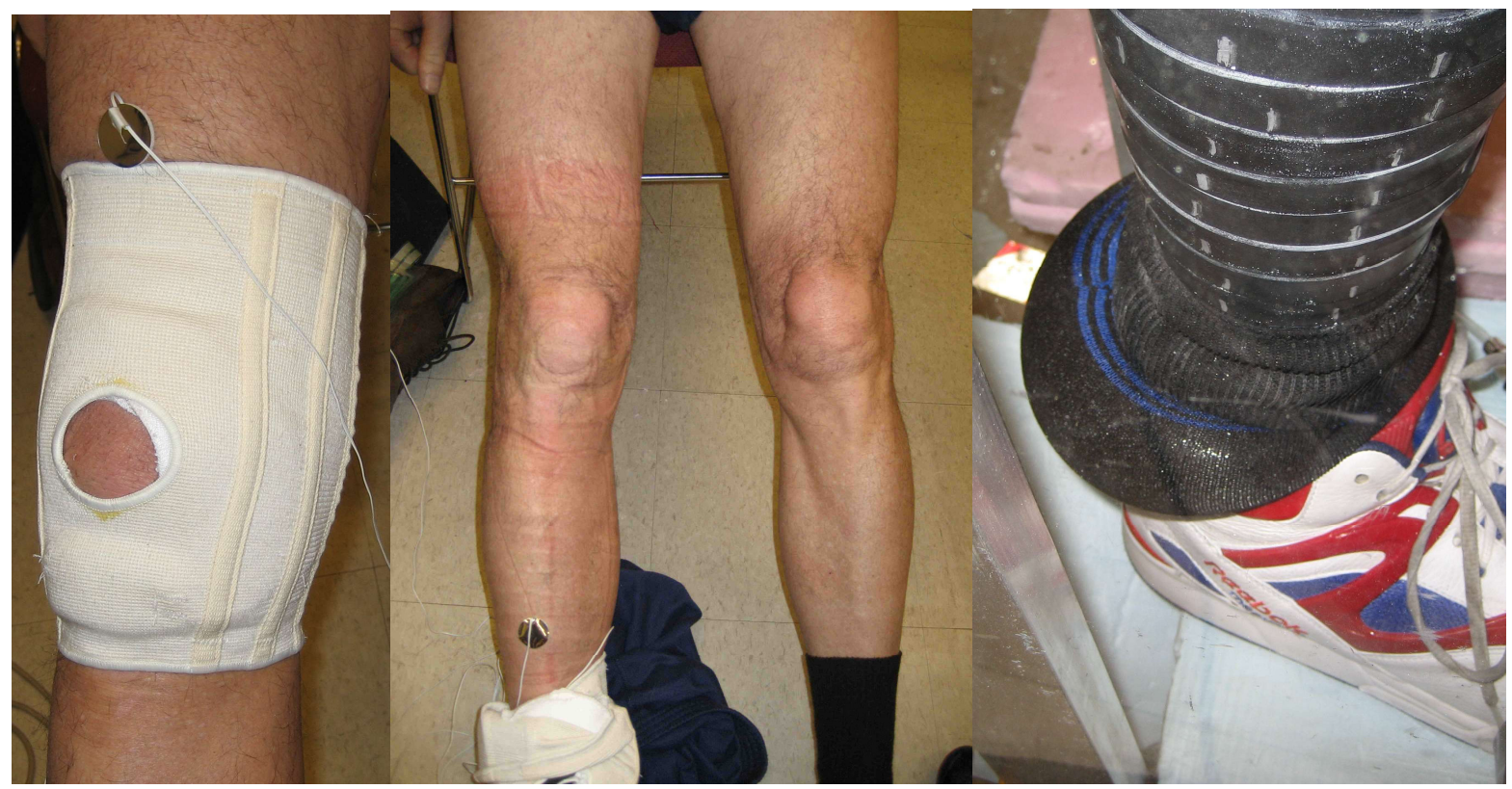

Figure 67: (left) Leg of Subject 2 in the knee brace Figure 68: (center) Legs of Subject 2 after the test with the knee brace Figure 69: (right) Sock of Subject 2 swelling outside the restraining layer

It should be noted that for both tests for this subject, the pressure in the chamber was not at the desired $-225 \mathrm{~mm} \mathrm{Hg}$ of underpressure. For the first test, the average pressure in the chamber was approximately $-200 \mathrm{~mm} \mathrm{Hg}$, and for the second test was approximately $-175 \mathrm{~mm} \mathrm{Hg}$. Part of the reason for this inability of the vacuum pumps to handle the load was that in each test for this subject the hybrid sock swelled out of the restraining layer (the basketball shoe), as can be seen in Figure 69, which placed considerable stress on, and then separated, the adhesive in the hybrid sock, causing it to leak. Therefore, the lack of edema, especially in the second test, may have been due in part to the less than desired underpressure the subject was experiencing, in addition to the use of the knee brace. 
Subject 5 (19 year old female, $1.72 \mathrm{~m}$ tall, mass of $61.2 \mathrm{~kg}$ ) finished the full hour with very little edema on the section of her leg covered by the neoprene bands, as seen in the figure below. However, examination of her foot showed considerable edema after the test ended. It is thought that the air hose connecting the hybrid sock to the atmosphere became sharply bent about halfway through the test (this is when the subject first stated feeling discomfort in her foot) due to the movement of the subject, which stopped the flow of air from the atmosphere into the sock, and consequently due to the small leak inherent in the design (it is impossible to create a perfect seal in the hybrid sock that stops $100 \%$ of air flow), she began to experience considerable underpressure on her foot. Within 24 hours much of the edema, seen in Figure 70 and Figure 71, was gone.

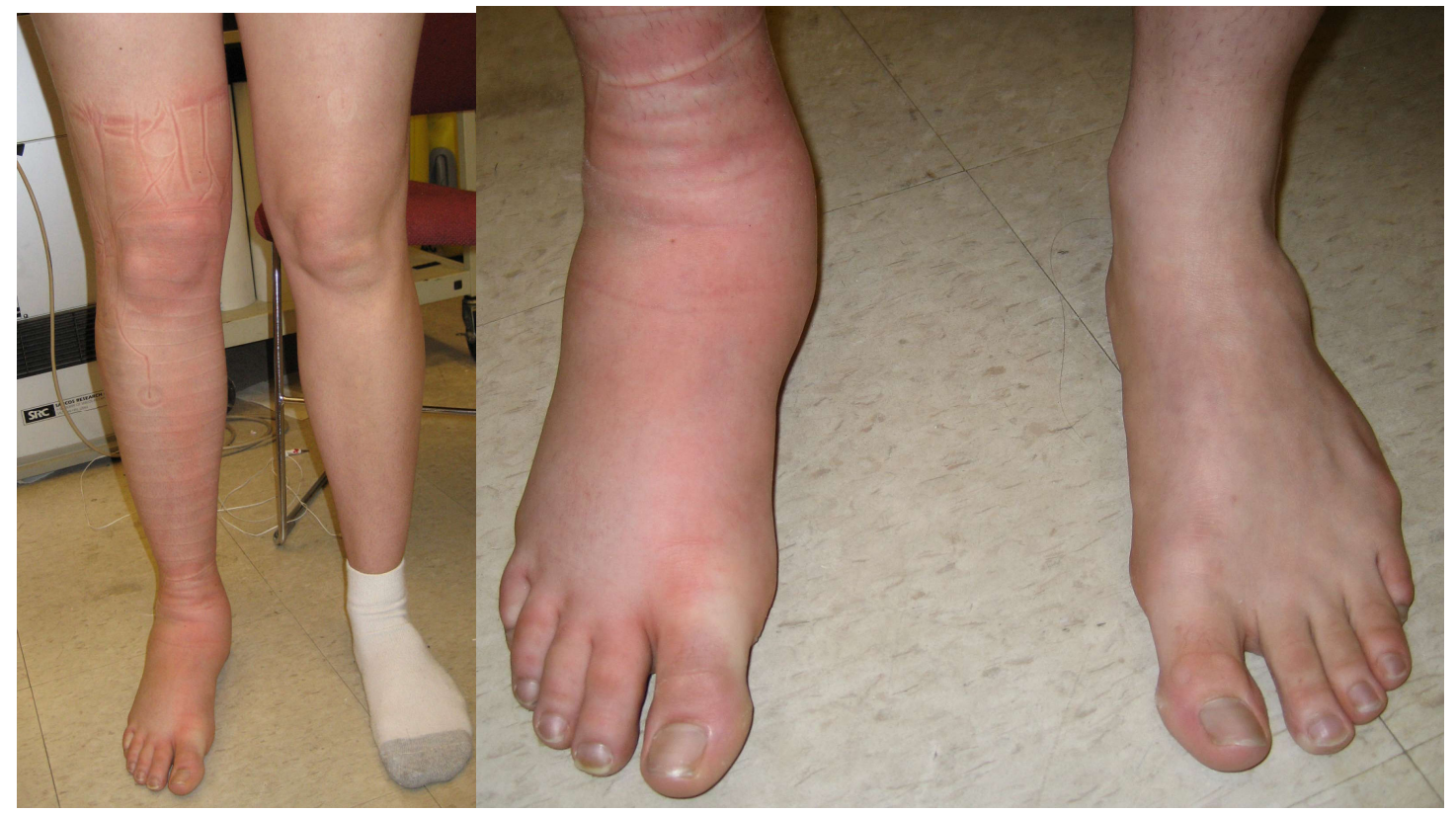

Figure 70: (left) Subject 5 after exiting the chamber

Figure 71: (right) Subject 5's feet after the test

Subject 6 (24 year old male, $1.82 \mathrm{~m}$ tall, mass of $63.5 \mathrm{~kg}$ ) completed the full hour-long test with little discomfort, but experienced considerable edema on both his foot and knee as seen in Figure 72 and Figure 73. In this test case, the hybrid sock failed to hold atmospheric pressure as the adhesive failed, so the subject was experiencing considerable underpressure on his foot. Additionally, in order to try to reduce the seal leak rate that plagued the previous subjects, duct tape was taped over the inextensible bladder to attempt to create a better seal, as can be seen in the following figure. ${ }^{25}$ However, when this subject was sealed, the duct tape may have provided a small tourniquet effect. Therefore, it is possible that when the neoprene bands were wrapped up the calf and knee, the blood slowly being squeezed up the leg by the wrapping process was unable to pass this tourniquet, and consequently some became trapped in the knee. Beyond this factor, the test director mistakenly

\footnotetext{
${ }^{25}$ The urethane coated inextensible bladder provided from ILC Dover by Dave Graziosi was tapered from the section clamped in the two metal rings, but still was typically an inch or two larger in diameter than the human thigh it was covering. Therefore, the seal had to be folded over to conform to the leg geometry, which was thought to lead to higher leak rates.
} 
allowed the pressure in the chamber to remain at $-240 \mathrm{~mm} \mathrm{Hg}$ for the vast majority of the test. Therefore, the subject was probably experiencing underpressure, as the bands are not calibrated to provide this level of pressure. The relatively small underpressure $(15 \mathrm{~mm} \mathrm{Hg})$ may have led to a slow onset of edema, hence the lack of discomfort. Like Subject 5, the swelling in Subject 6 had largely subsided within 24 hours.

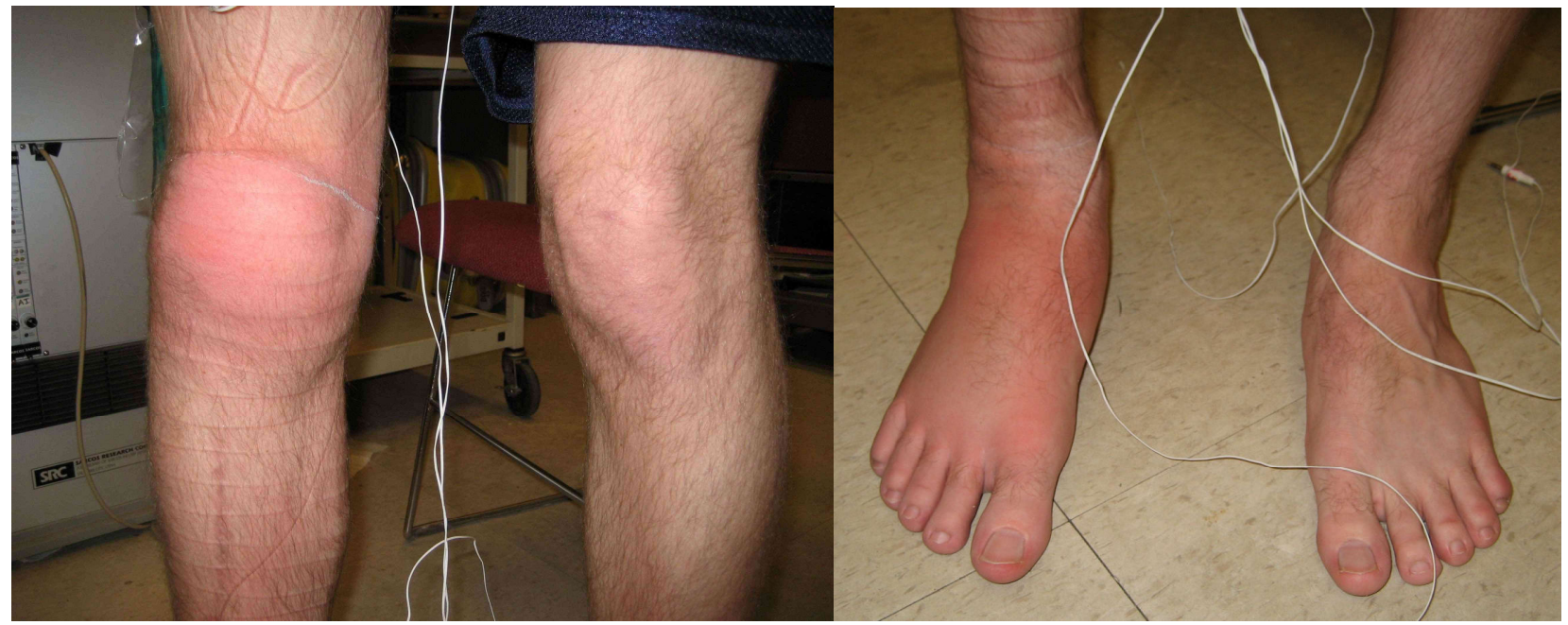

Figure 72: (left) Edema on knee of Subject 6 Figure 73: (right) Edema on foot of Subject 6

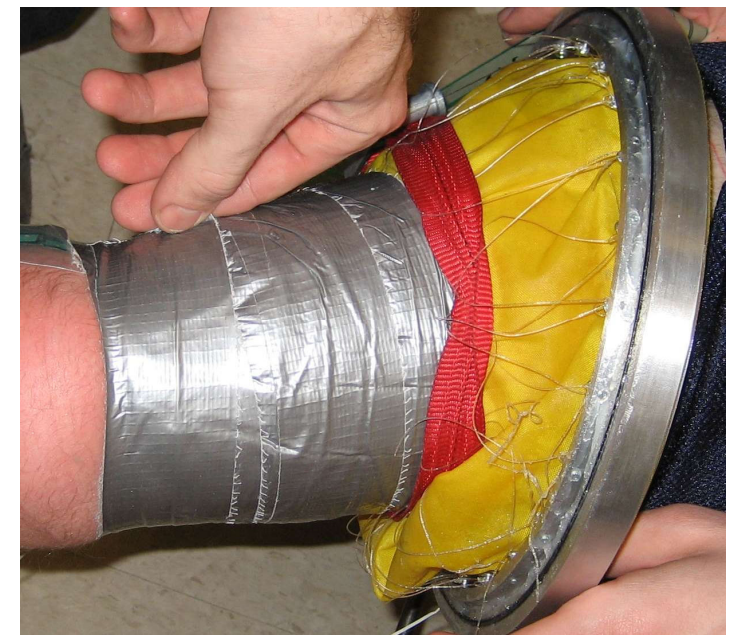

Figure 74: Duct tape over the seal

Subject 7 (19 year old female, $1.72 \mathrm{~m}$ tall, mass of $72.6 \mathrm{~kg}$ ) was unable to complete the experiment, ending after approximately 42 minutes, due to a physiological reaction. Unlike Subject 4 , it is highly unlikely she was experiencing underpressure, as she spent considerable time in the chamber, but exited with little edema, as seen in the following figure. A more thorough investigation of her test data is available in Appendix $\mathrm{O}$. 


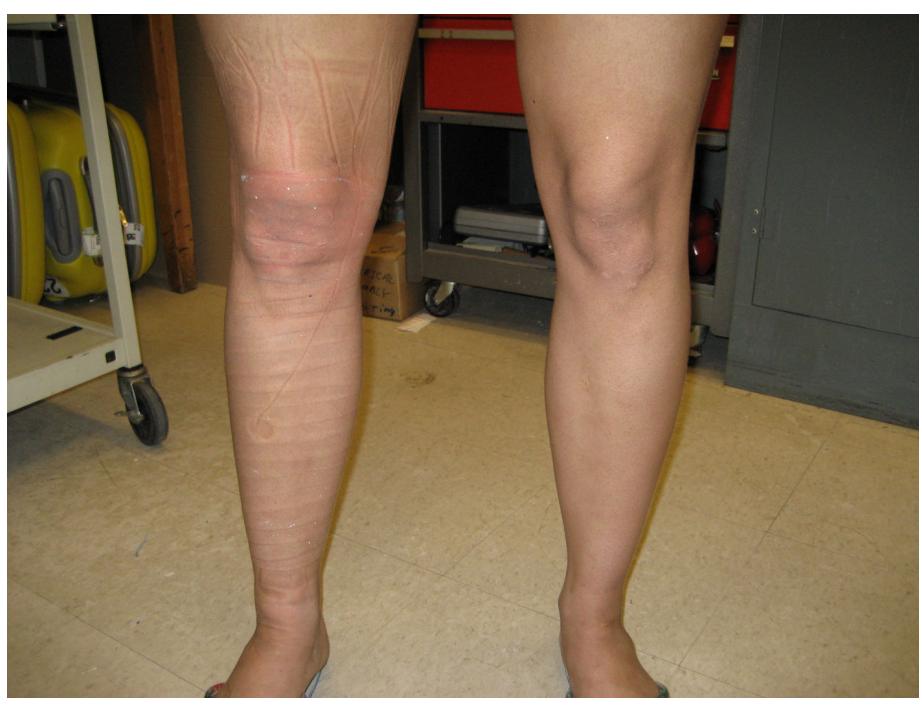

Figure 75: Leg of Subject 7 after test

\subsection{Main study results}

Physiological data was analyzed using SYSTAT 11 for significance in a linear regression model with the pressure in the chamber as the independent variable. Significance was set at $\mathrm{p}<0.05$.

All data was normalized to the 6 minutes of baseline measurements taken prior to entering the chamber. Therefore, the data presented in this section (other than the TekScan pressure measurements and qualitative discomfort measurements) will be presented in either percentages change from baseline measurements (heart rate and blood pressure) or changes in degrees Celsius from the baseline (skin temperature). Many of the figures presented display the averages of the 6 tests conducted, so results should be interpreted with caution based on the small sample size. In some cases the data from one subject caused statistical significance, and these situations are all clearly noted in the text. In all figures, the time $\mathrm{t}=0$ is when the chamber pressure first reached -225 $\mathrm{mm} \mathrm{Hg}$.

\subsubsection{Discomfort measurements}

The titles of the graphs in this section indicate the number of the subject completing the experiment, and for Subject 2 further specifies which test was being completed. Subject 2 ended his first test early (after 50 minutes) due to a scheduling commitment, and Subject 7 experienced a physiological reaction, which is why those tests end before the hour is completed. Only Subject 3 experienced significant discomfort, which was mostly due to the lack of pressure on her foot. 
1
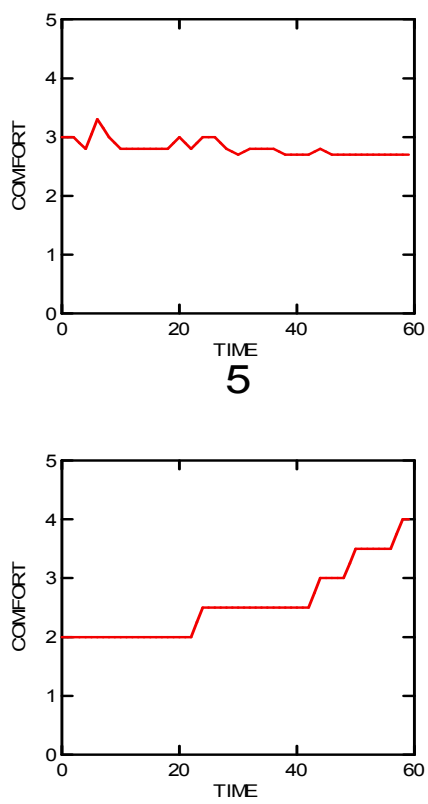

2 Test 1
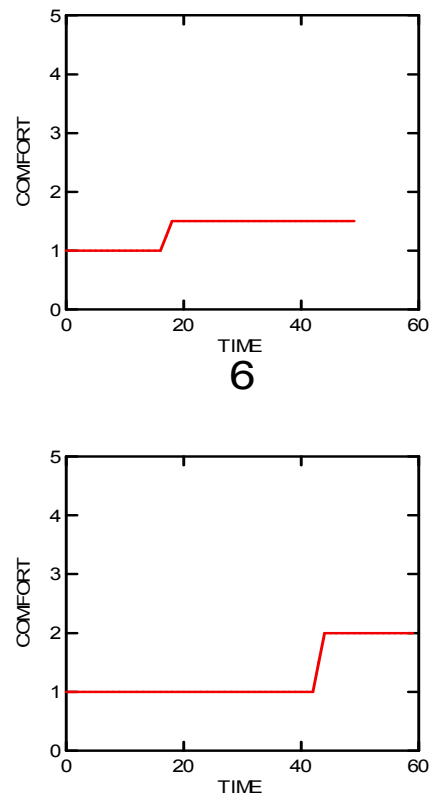

2 Test 2
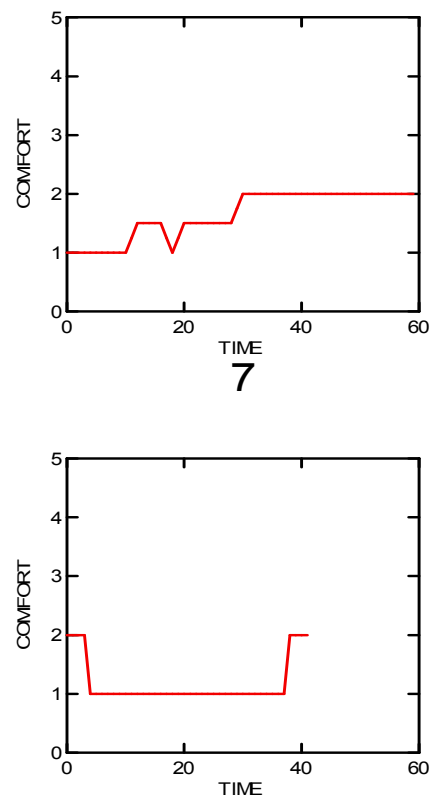

Figure 76: Discomfort measurements by subject and trial

\subsubsection{TekScan results}

The following readings were taken of each subject while he or she was wearing the neoprene bands to protect against the $-225 \mathrm{~mm} \mathrm{Hg}$ underpressure of the chamber. The error bars represent one standard deviation away from the average recording, using the method for determining the standard deviation previously defined in the initial portion of Chapter 4 . The readings are based on the averaging of the data recorded every 5 minutes, with some rows removed, as in a few cases the TekScan sensor was not placed appropriately, and some of the sensor was not located under the neoprene wrap. Additionally, all values of $0 \mathrm{~mm} \mathrm{Hg}$ (indicating the sensor had no recording) were removed, as they improperly influenced the average pressure.

Measurements were also recorded for each subject in the chamber at normal atmospheric pressure. The readings from Subjects 1 and 6 were taken at the end of the experiment, while those from the other subjects were taken prior to the depressurization of the chamber. 


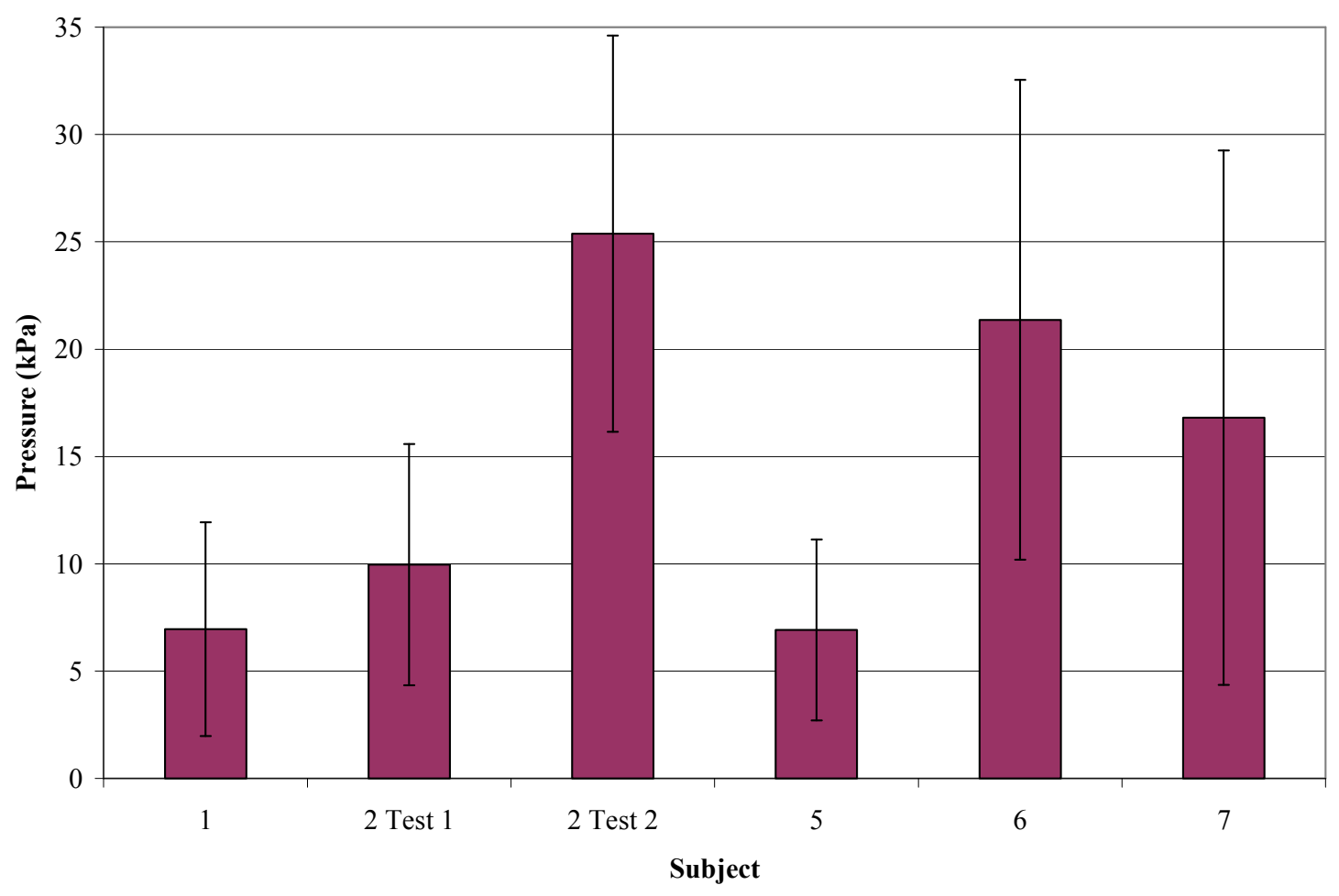

Figure 77: In-chamber pressure readings from main study

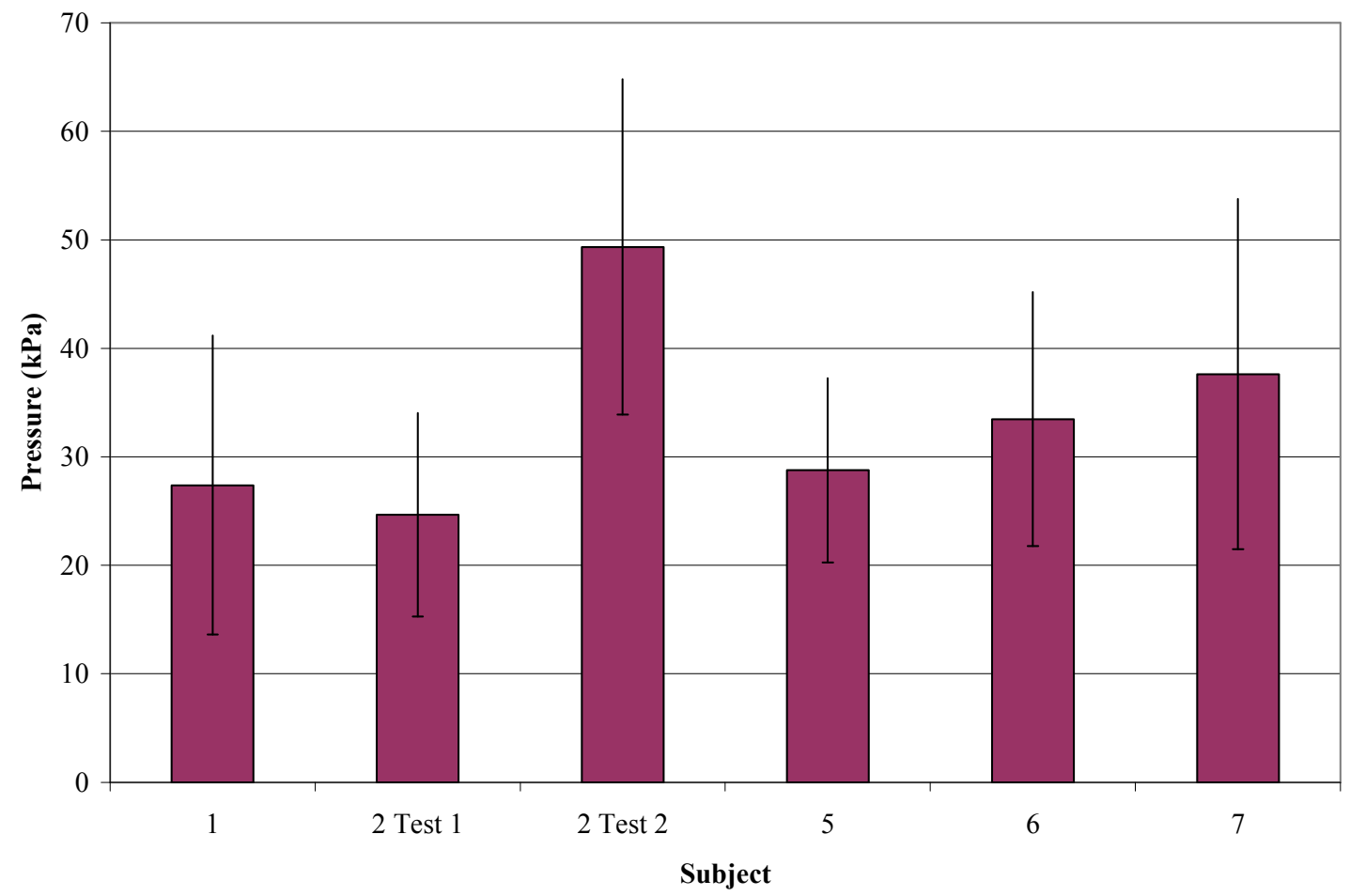

Figure 78: Pressure measurements from subjects under MCP at normal atmospheric pressure 


\subsubsection{Heart rate}

In this study, there was no data available from Subject 1, as the monitor did not record her heart rate for an unknown reason. Figure 79 represents the averaged data from the remaining subjects, normalized to their individual baseline heart rates and the error bars shows the \pm standard error of the five subjects. However, the data from Subject 5 varied considerably from the other 4 data sets, as seen in Figure 80. With the removal of Subject 5's data, there is no statistically significant change in heart rate.

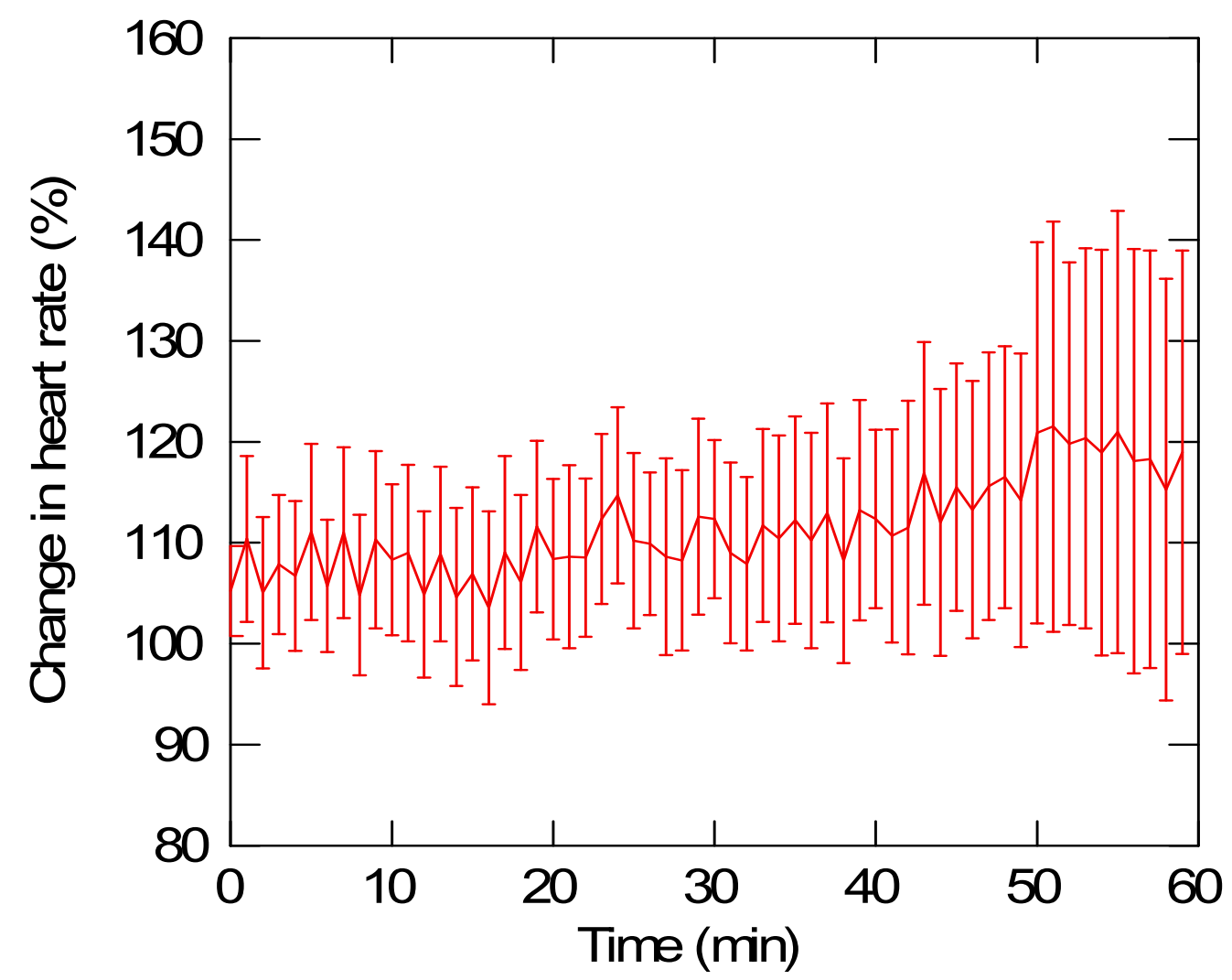

Figure 79: Change in heart rate for all subjects

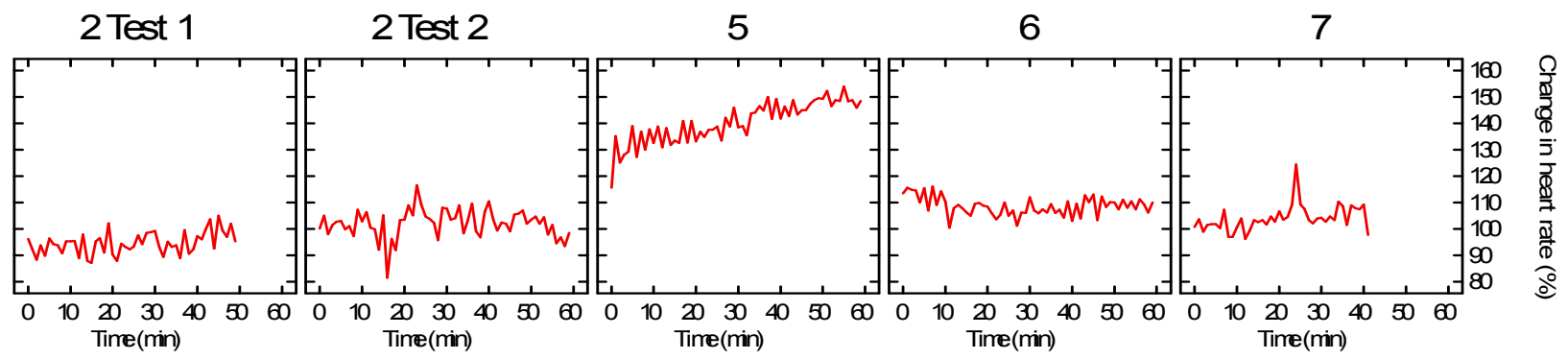

Figure 80: Change in heart rate for the subjects individually 


\subsubsection{Blood pressure}

As noted earlier in this chapter, the blood pressure of the subjects was taken and recorded every 2 minutes. However, despite the efforts of the subjects not to talk during these measurements, many of these recordings were inaccurate (systolic readings lower than diastolic readings, readings in the single digits). Those readings were removed from each of the subjects, but given the lack of reliability in the measurements by the system, it is unclear whether the data presented here is entirely accurate, as some of the outlier data points may be due to measurement error and do not represent the actual blood pressure of the subjects. The data presented in the following figures shows the changes in the blood pressure of all six subjects, normalized to their individual baseline blood pressures and the error bars shows the \pm standard error of the six subjects. The changes in both the systolic and diastolic blood pressure are significant. However, the individual blood pressure changes of the subjects are quite diverse as seen in Figure 81 and Figure 82.

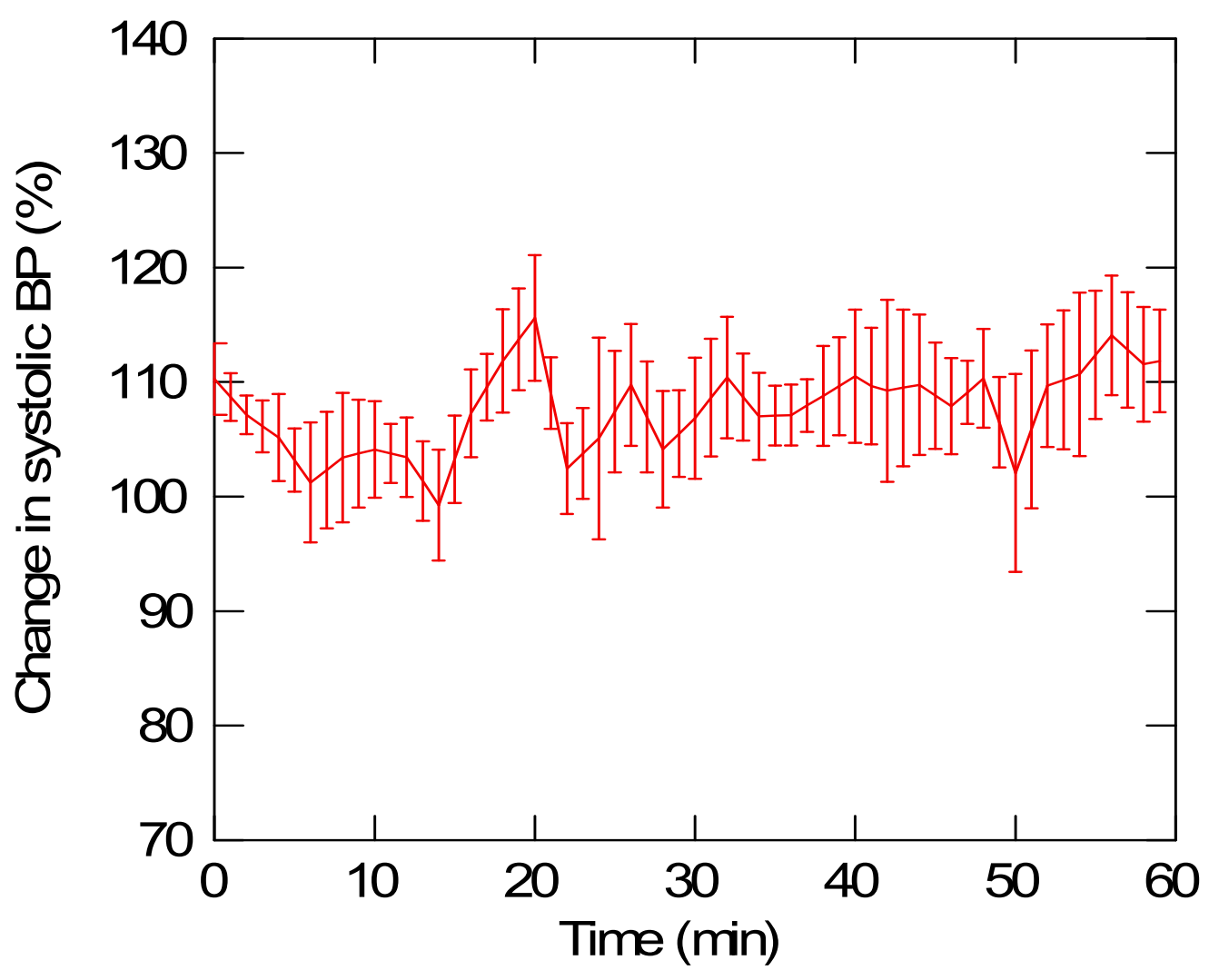

Figure 81: Average change in systolic blood pressure 


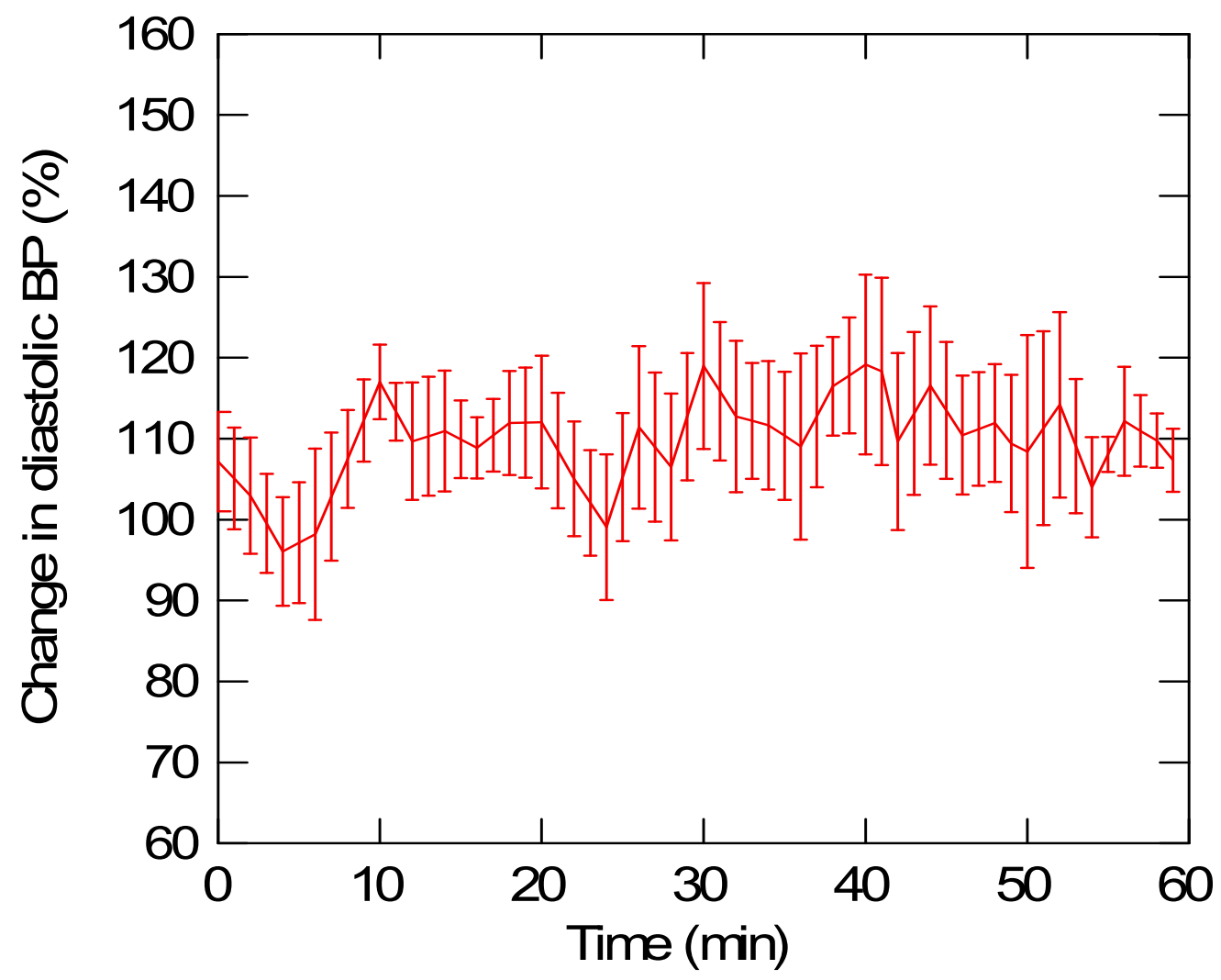

Figure 82: Average change in diastolic blood pressure

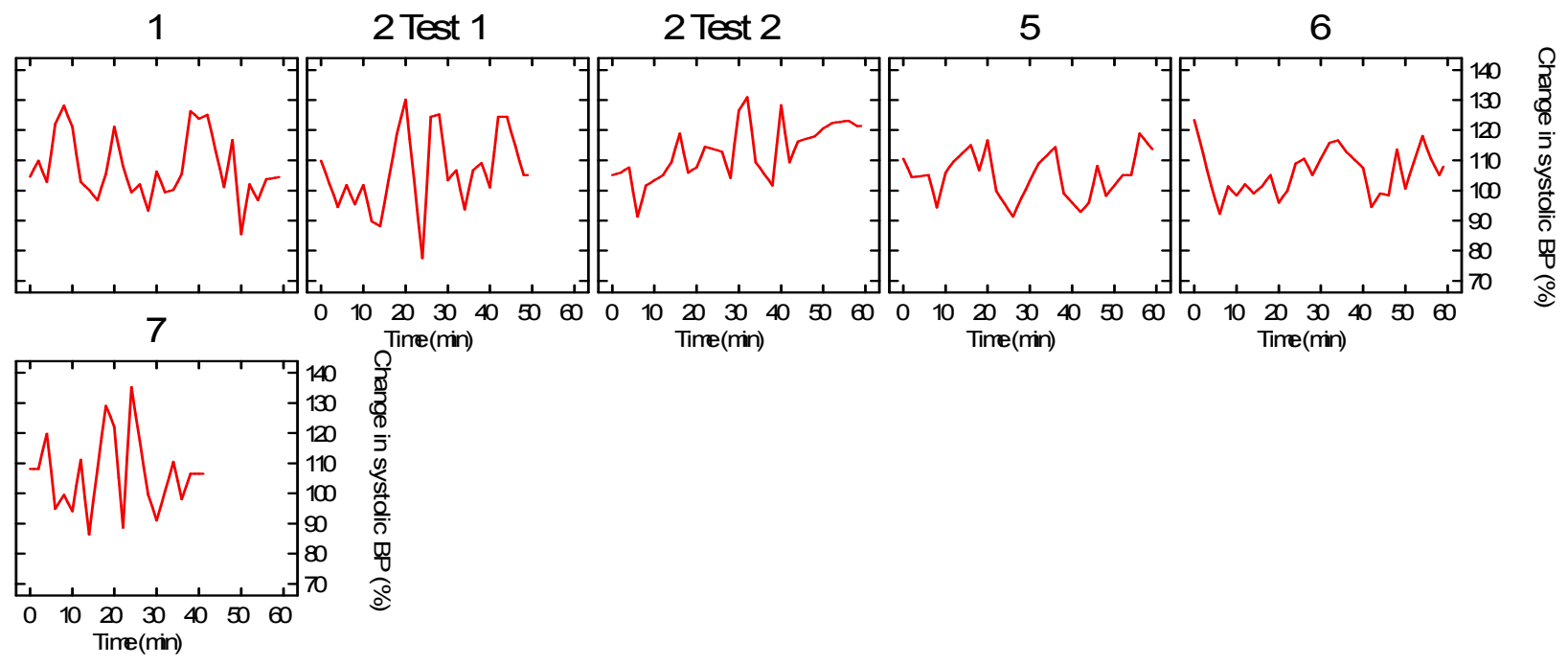

Figure 83: Change in systolic blood pressure for each subject 


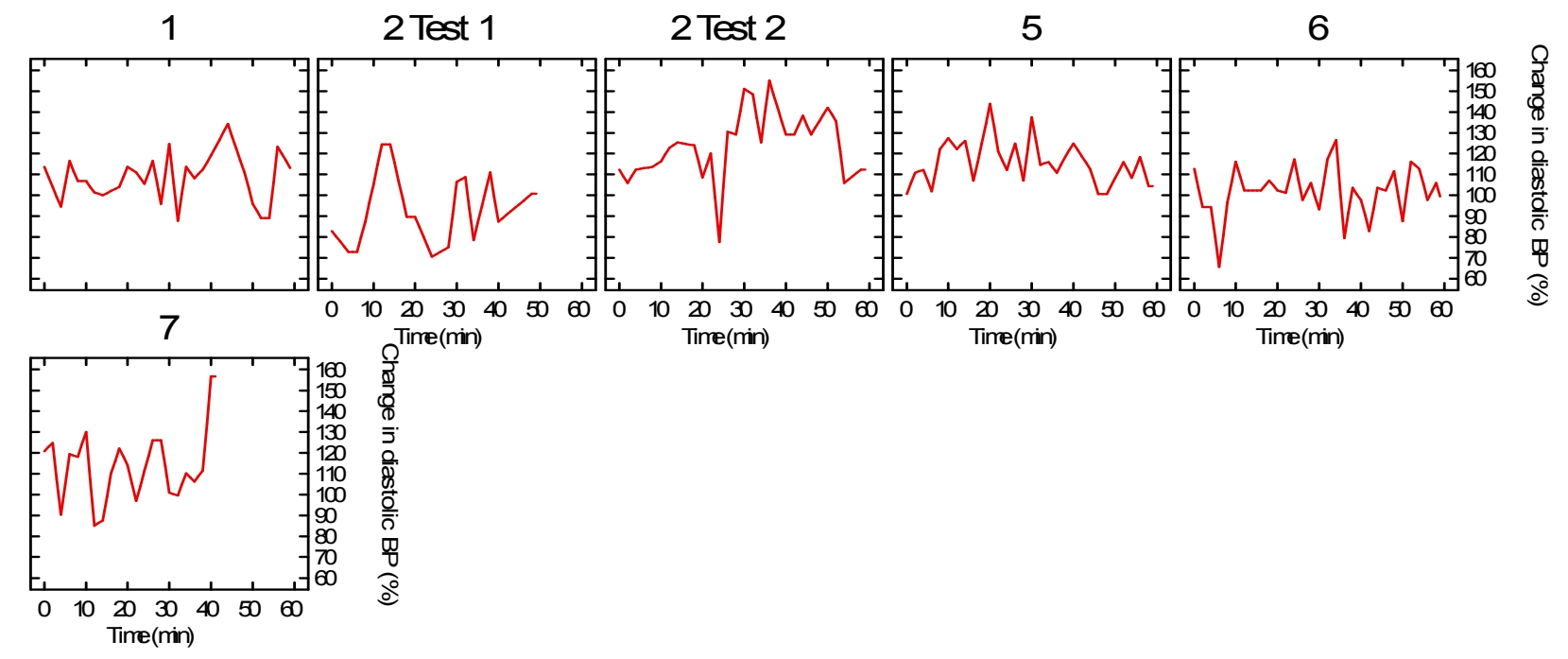

Figure 84: Change in diastolic blood pressure for each subject

\subsubsection{Skin temperature}

The skin temperature changes are presented based on the difference from the baseline temperature recordings. In most cases, when the test began, the subject's legs were warmer, as seen in Figure 85. This figure shows a relatively small change (no more than $1{ }^{\circ} \mathrm{C}$ ) on the average, but the experiences of the subjects varied widely, so the data for each of the sensors shall be presented separately. Sensor 1 was placed on the anterior of each subject's right shin, sensor 2 on the posterior of each subject's right thigh, sensor 3 on the anterior of each subject's right thigh, and sensor 4 on the anterior of each subject's left (out of chamber) thigh. 


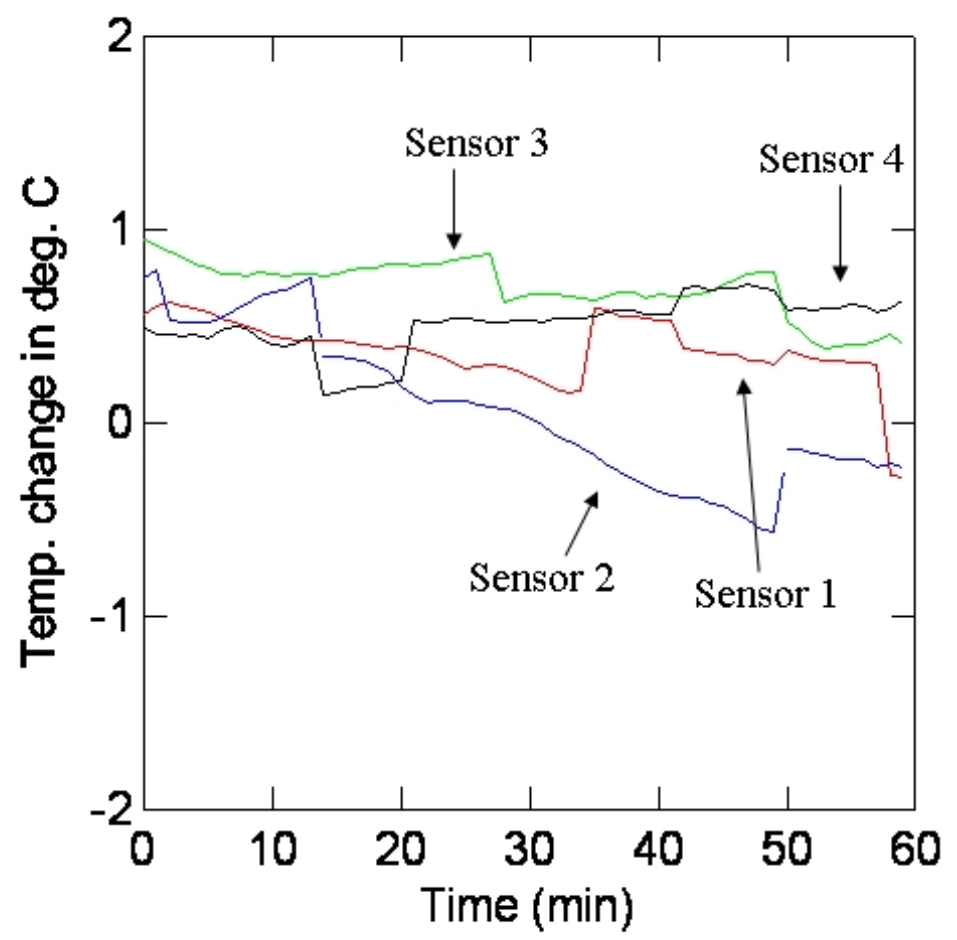

Figure 85: Skin temperature change for each sensor based on the averages of the subjects

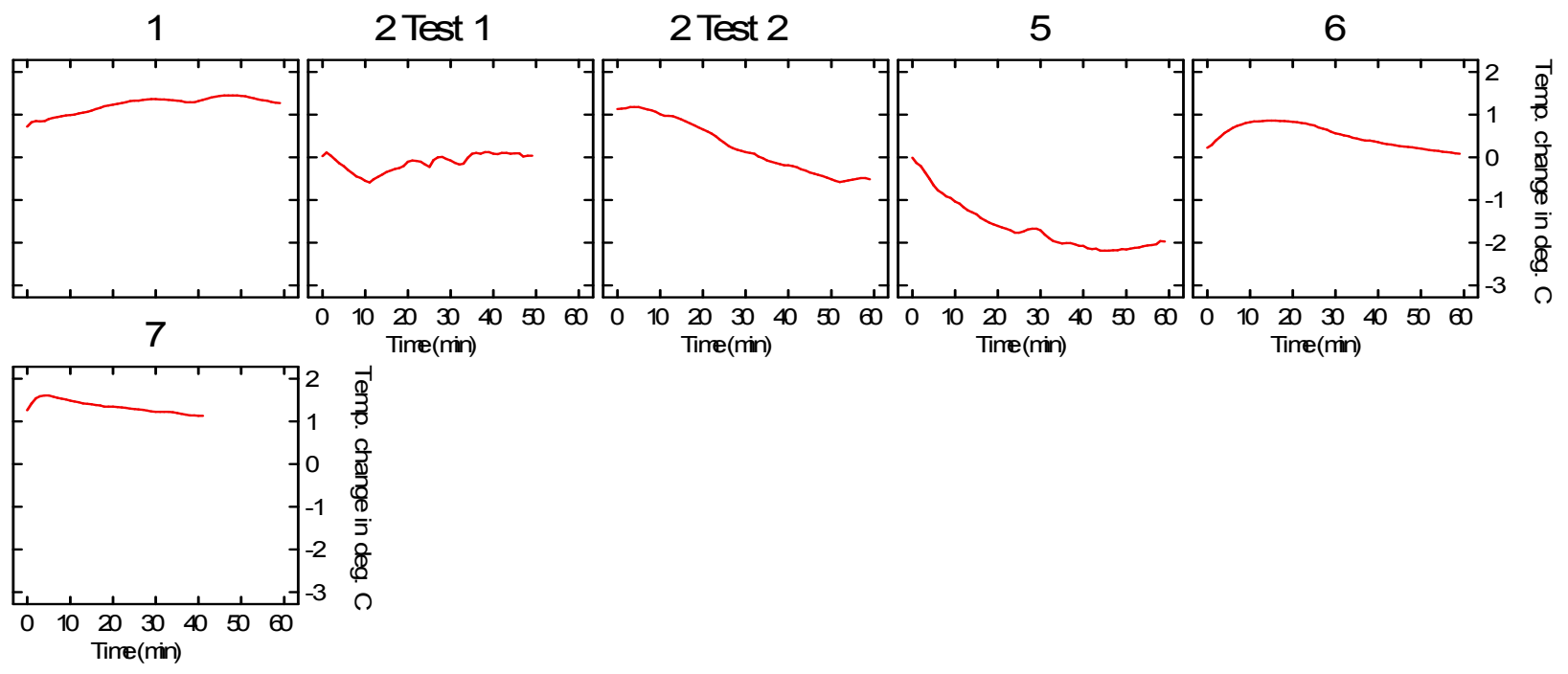

Figure 86: Temperature sensor 1 (right shin anterior) readings 


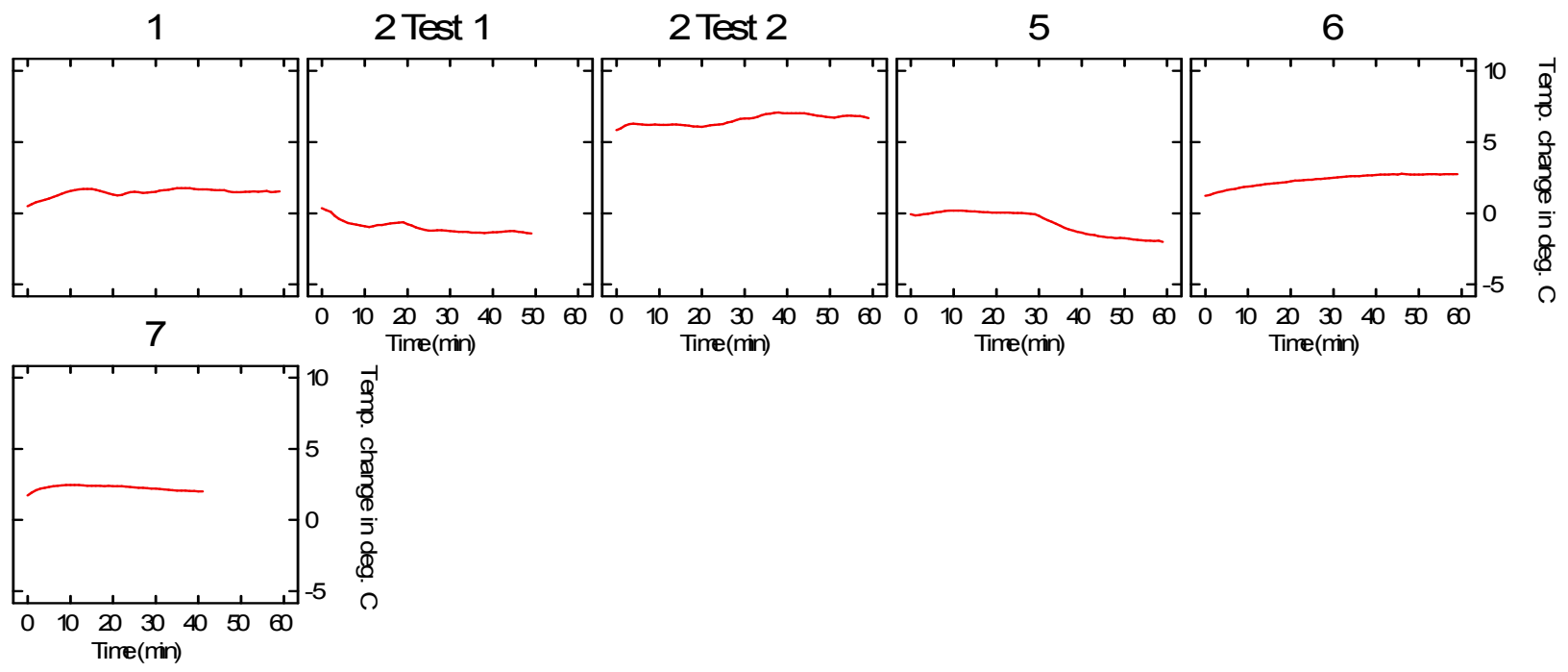

Figure 87: Temperature sensor 2 (right thigh posterior) readings

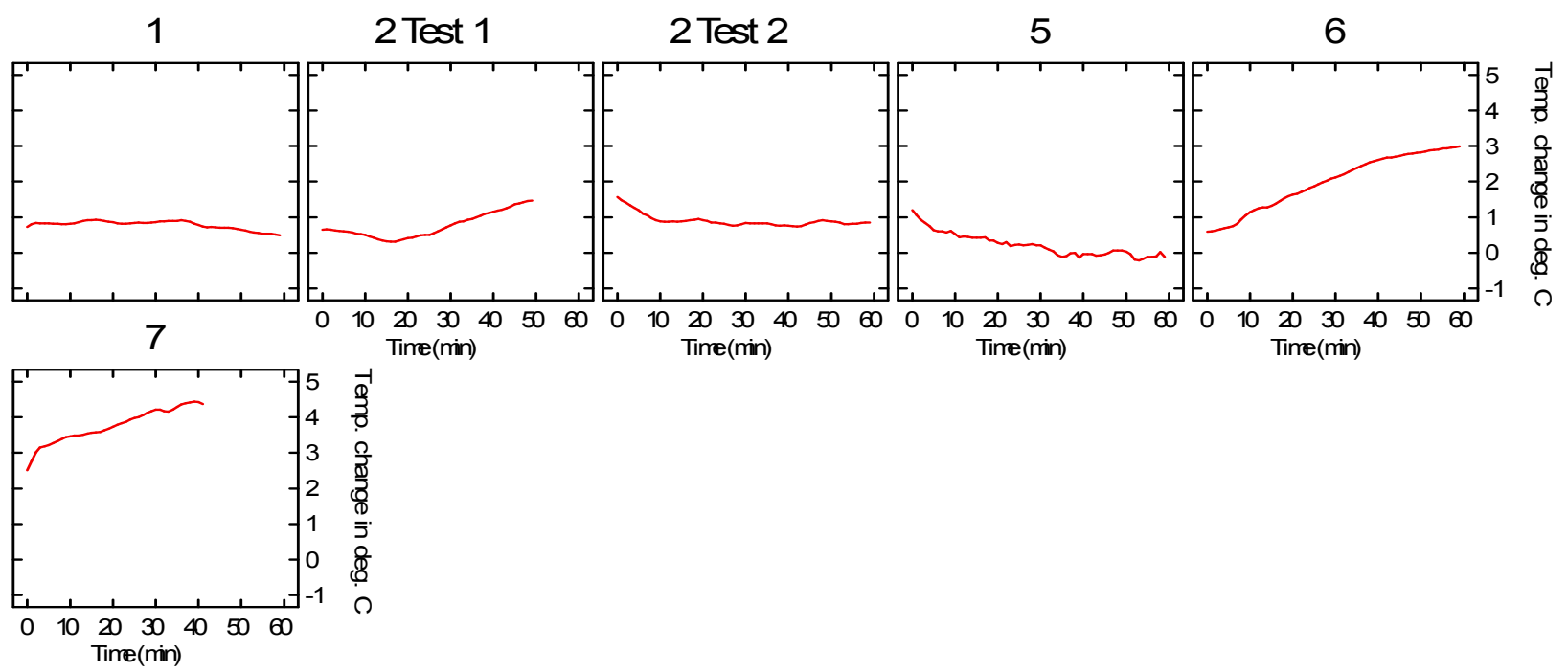

Figure 88: Temperature sensor 3 (right thigh anterior) readings 


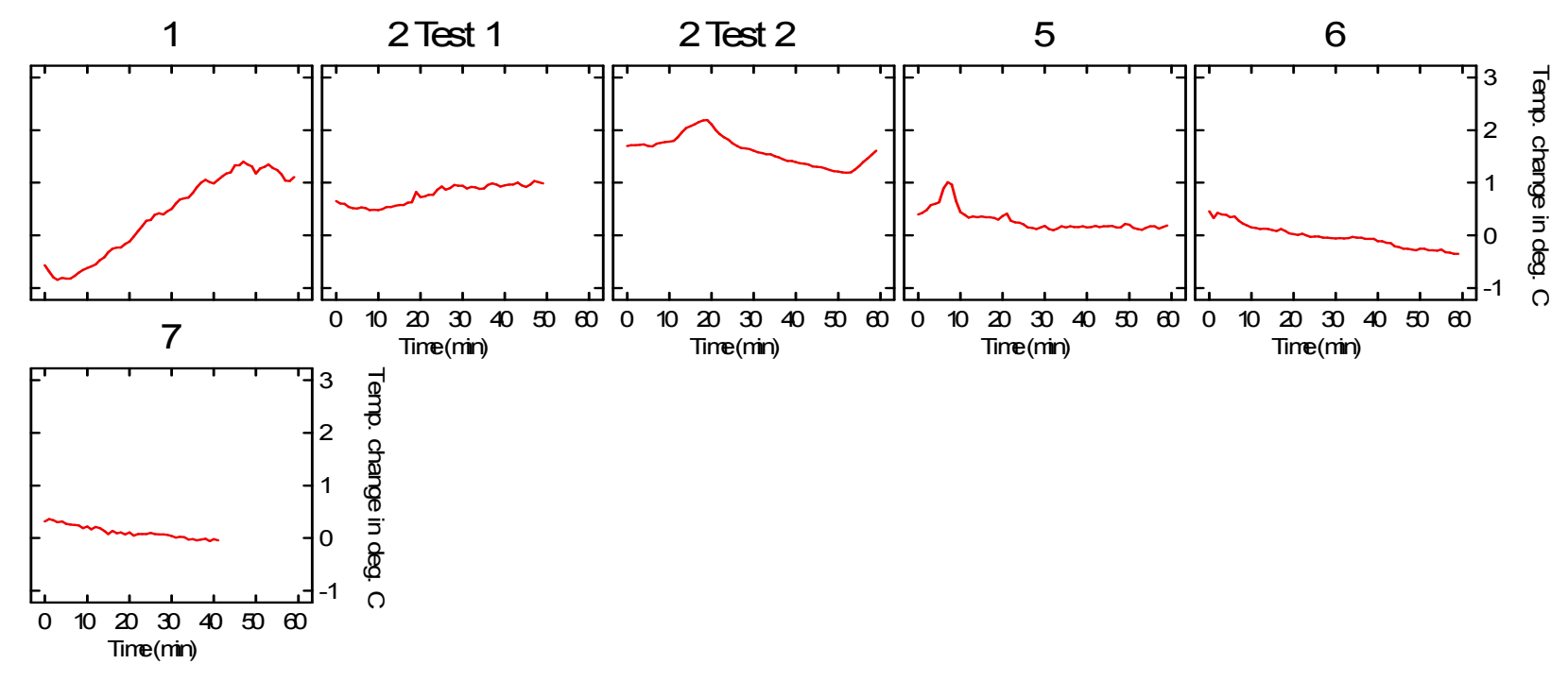

Figure 89: Temperature sensor 4 (left thigh anterior) readings

\subsection{Main study discussion}

\subsubsection{Effectiveness of neoprene wrap}

These tests are the highest levels of MCP (225 mm Hg) applied for the longest duration (1 hour) over a section of the body larger than the calf. From the data it appears that the neoprene bands are adequately protecting the leg, other than sections around the kneecap. The only major edema in areas protected by the neoprene bands was on Subject 4's knee, and the possible tourniquet caused by the duct tape as well as the additional underpressure experienced by that subject are mitigating factors for that edema.

Subject 2's multiple experiments, one without a knee brace and one with a knee brace used to circularize the cross-sections of the knee, indicate that with a relatively simple modification, MCP can effectively protect the entire leg between the ankle and the thigh. However, as noted previously, Subject 2 experienced less than desired underpressure in both situations, and was experiencing the least underpressure in the test with the knee brace, which prevents a stronger conclusion from being drawn regarding the effectiveness of the knee brace.

In Sim's experiments 3 of the 5 subjects noted significant discomfort, although the hybrid sock for one of those subjects failed as well. However, the relative lack of discomfort in the current experiments compared to the Sim experiments is more generally due to the improvement of the double wrap over the single wrap, and the increased comfort of the seal, which did not tourniquet the subject, as in Sim's experiments [14]. 
The double wrap, while more comfortable than the single wrap, still can be improved. As seen in the figures presented earlier in this chapter summarizing the experiences of the subjects in the main study, the neoprene wrap still creates marks on the skin based on the edge of the layers. The bands slipped multiple times, leaving sections of skin exposed directly to the underpressure of the chamber or creating sections of overpressure. Either way, it affected subject comfort levels. Donning a more operationally capable garment should reduce the subject discomfort levels significantly.

\subsubsection{TekScan readings}

The readings from the TekScan sensors were considerably different than those from the Sim experiments, in which the measurements were somewhat less than $225 \mathrm{~mm} \mathrm{Hg}(30 \mathrm{kPa})$. Since in Sim's experiments the sensors were placed over the anterior of the shin, this was not entirely unexpected [14]. In this experiment, the TekScan sensor was placed on the exterior of the thigh for each subject, and the readings were expected to be approximately $225 \mathrm{~mm} \mathrm{Hg}$ due to the fat content in this area of the body, which allows it to circularize more than the bony shin. The tests detailed in Chapter 4 attempted to discover why the readings were so much lower than expected, but still were unable to provide a method for more accurately determining pressures, as seen by the low readings presented in the previous section. To understand fully the differences between the these tests and those conducted by Sim the four changes made between the experiments will be stated here:

1. The lower calibration point for these experiments was $150 \mathrm{~mm} \mathrm{Hg}$ in this experiment, while it was $100 \mathrm{~mm} \mathrm{Hg}$ in the Sim experiment [14].

2. The testing rig used for calibration was the soft inner cylinder, while Sim used the flat plate [14].

3. The TekScan sensor was typically partially covered by the inextensible bladder in these tests, while the remainder of the sensor was directly pressurized by the neoprene bands. For Subject 2's second test in this thesis, the TekScan sensor was placed under the knee brace and the inextensible bladder. In Sim's experiments, only the neoprene bands covered the sensor [14].

4. As noted in the paragraph above, the sensor was placed on the exterior of the thigh, as opposed to the anterior of the shin.

Changes 1 and 2 should have made the tests more accurate, not less. Calibrating within a closer range to the desired pressure (up to $75 \mathrm{~mm} \mathrm{Hg}$ ) should provide more accurate results [23] and Chapter 4 indicated the soft cylinder would be more accurate for the given placement on the body in these experiments. Therefore, only changes 3 and 4 remain as the cause of the low pressure readings, but it is still unclear why the results from these tests differed so significantly from those conducted by Sim.

However, the data presented regarding the tests taken at atmospheric pressure and $-225 \mathrm{~mm} \mathrm{Hg}$ pressure indicate that the chamber pressure is causing the low readings. A few other readings were 
taken while the chamber was at partial underpressure (approximately $-150 \mathrm{~mm} \mathrm{Hg}$ and $-75 \mathrm{~mm} \mathrm{Hg}$ ) and they fall within the boundaries between the normal atmospheric pressure and the $-225 \mathrm{~mm} \mathrm{Hg}$.

In Sim's tests, the TekScan readings were somewhat lower than expected (on average around 20-25 $\mathrm{kPa})$ on the anterior of the shin. Sim reasoned that the pressure measurements were accurate, and that the lower pressures were caused by the concavities of the shin [14]. However, given the difference in measurements between the sensor data at atmospheric pressure and $-225 \mathrm{~mm} \mathrm{Hg}$ of pressure, it is possible that the wraps used by Sim were actually overpressurizing the subjects. The anterior of the shin is a hard, bony surface, with a relatively small radius of curvature due to the bone. From equation 1, areas of the body with smaller radii of curvature should have larger pressures, which would indicate that Sim was overpressurizing the anterior of the calves of his subjects. However, given the small sample size and the limitations of the TekScan system

discussed in Chapter 4, this conclusion should be interpreted with caution, as other factors may be influencing the pressure measurements that are not understood.

A system to record a more precise measure of the pressure applied by the neoprene bands is imperative for accurately characterizing the effectiveness of the neoprene bands. Without this data it is impossible to know for certain if the relative lack of edema is based on the production of the desired average pressure by the neoprene bands $(30 \mathrm{kPa})$ with minimal variation around a given cross-section, or because the legs are more tolerant to underpressure than the current target variation of $1.6 \mathrm{kPa}$.

\subsubsection{Heart rate}

There was no significant change in heart rate, which indicates that the neoprene bands adequately protected the subjects from the effects of discomfort. In Sim's study, there was no significant change in heart rate in the chamber with or without the use of the neoprene bands to protect the lower leg [14], but in this test a greater portion of the body (a full leg as opposed to just the calf) was placed in the chamber. Reddig, et al, found an increase in heart rate after exposing the full lower body of subjects to $-40 \mathrm{~mm} \mathrm{Hg} \mathrm{[24].} \mathrm{The} \mathrm{larger} \mathrm{surface} \mathrm{area} \mathrm{exposed} \mathrm{to} \mathrm{negative} \mathrm{pressure}$ probably promotes the greater shift of blood volume, but it is unclear what the threshold, either the full lower body or just one leg, is to experience a response. The only large change during the test in heart rate was experienced by Subject 5, but is unclear why her heart rate increased.

\subsubsection{Blood pressure}

The changes in the blood pressure in this thesis were significant, but it is not clear as to whether they are representative of the experiences of the subjects. As noted previously, some of the data points recorded were clearly inaccurate (single digit blood pressures, systolic blood pressures lower than diastolic blood pressure) and they were removed. However, due to the readings being recorded every two minutes, and some readings being inaccurate, a higher than average reading can drive the data significantly, as interpolation was used between points for the graphs in the previous section. The manner in which the readings fluctuate (note especially Subject 2 Test 2 in Figure 84) indicates that the data may be inaccurate. 
Recording blood pressure constantly on an arm causes a change in the readings themselves, as the application of pressure repeatedly causes the pathways of blood in the arm to contract, changing the blood pressure. The arm used to record blood pressure was changed every 10 minutes, but it still may have had an effect on the data. Additionally, the subjects were encouraged not to talk during readings, but some talking and movement may have affected the results. Further studies, preferably with different, more accurate equipment, is needed to show whether this significant result is real.

\subsubsection{Skin temperature}

There were some significant differences between the baseline readings and the start of the temperature readings. For instance, Subject 7 had a large $\left(\sim 1.5^{\circ} \mathrm{C}\right)$ temperature increase on her shin anterior and an even larger $\left(\sim 3^{\circ} \mathrm{C}\right)$ increase on her right thigh anterior. Subject 2 experienced the largest increase prior to the start of his second test $\left(\sim 5^{\circ} \mathrm{C}\right)$ on his right thigh posterior. In Sim's study some of the subjects experienced on the order of a $2{ }^{\circ} \mathrm{C}$ change between the baseline measurements and the start of the study. Given that wrapping the entire leg is a longer process which restricts blood flow to a larger portion of the body, it is not unsurprising that larger changes in temperature were recorded in these experiments. As in Sim's experiments, no meaningful conclusions can be drawn regarding skin temperature, as the subjects had very different responses to their time in the chamber [14]. It should be noted that a material that allowed the user to sweat (which the neoprene does not) would allow the skin temperature of the subjects to stabilize, and might provide more consistent readings than in the current study.

The $\sim 2.5^{\circ} \mathrm{C}$ rise recorded by sensor 3 for Subject 6 could have been indicative of the edema on his knee. In retrospect, given the edema on Subject 6's knee and foot as well on Subject 5's foot, the placement of the temperature sensors was not ideal. Placing sensor 1 on the foot of the subject and sensor 3 on the subject's kneecap would have been far more effective in discovering edema than the locations in this study (sensor 1 on the shin anterior and sensor 3 on the thigh anterior). The readings on temperature sensor 1 actually dropped over time for both of the subjects who experienced edema in their foot, indicating it was not measuring this swelling.

\subsection{Experimental limitations}

The hybrid socks affected the results of 4 of the 6 tests, by leaking at a high enough rate that the subject was experiencing insufficient underpressure in the chamber, or by failing to pressurize the foot at all, causing the subject to feel considerable underpressure (up to $-225 \mathrm{~mm} \mathrm{Hg}$ ). In order to more accurately determine the effects of MCP on the human body, the foot must be properly pressurized to eliminate unwanted additional variables. One item of future work should be to develop a more robust design to protect the human foot.

Other measures that might have provided greater insight into whether the subjects were experiencing underpressure, such as Laser Doppler flow meters and strain gages were unavailable for this study. These devices can measure the blood flow into an area of edema directly, and are superior measures to heart rate, skin temperature, and blood pressure, which are all indirect measurements of edema. 
Additionally, the small number of subjects $(n=6)$ restricted the power of statistical analysis. One subject in some cases (notably Subject 5 in heart rate) deviated considerably from the other subjects. Testing MCP on more subjects would provide greater power for determining the typical experience and would help answer the question regarding whether the significant change in blood pressure is real, or simply an effect of inaccurate data.

\subsection{Experimental testing conclusions}

In order to accurately pressurize the human body via MCP, the measurements of the human body must be taken when it is under MCP, otherwise the subject will experience underpressure. The reason for this requirement is that the human body "deforms" or "circularizes" under pressure, as the fat and muscle under the skin compact in order to distribute the pressure more evenly.

Four tests of one hour with a full leg pressurized via MCP in a chamber at approximately $-225 \mathrm{~mm}$ $\mathrm{Hg}$ (relative to atmospheric pressure) were completed, while a fifth ended at 50 minutes due to a scheduling conflict. These tests were conducted at a higher pressure $(-225 \mathrm{~mm} \mathrm{Hg})$ for a longer duration (1 hour) with more of the body covered than had ever been conducted previously. The neoprene bands appear to be effectively protecting the leg, with the exception of the concavities around the knee, from the underpressure in the chamber. One test with a knee brace used to fill these concavities with material to allow the neoprene bands to pressurize this area of the body more effectively was successful, but should not be considered conclusive due to the limitations of that test (the subject was experiencing overpressure throughout the test). More tests need to be conducted with some countermeasures around the knee to discover the best method of pressurizing this area of the body.

As the experiences of the subjects in the chamber varied ( 3 of the 6 tests were conducted at chamber pressures other than $-225 \mathrm{~mm} \mathrm{Hg}$ ), more tests should be conducted at the desired pressure to ensure statistically significant results for the physiological measurements. Two subjects experienced physiological reactions to the experiment, one for reasons of underpressure, while the cause of the other reaction is unclear. Further studies would provide greater insights into the reasons for these reactions. 


\section{Chapter 7: Conclusions and future work}

\subsection{Conclusions}

The conclusions of this thesis are multi-fold, as it has contributed to the knowledge of MCP in the areas of requirement definition, theoretical modeling, and experimental testing.

This thesis sets requirements for the fabric and closure mechanism in order to design a working BioSuit prototype. It also provides a compendium of the complete BioSuit system level requirements, which had not been centralized previously. By using common, everyday devices such as roller blades to produce $30 \mathrm{kPa}(225 \mathrm{~mm} \mathrm{Hg})$ of pressure, the testing proves that this pressure requirement is realistic.

The theoretical modeling provides some insight into the maximum capability of MCP to provide consistent pressure, and multiple methods for conducting the calculations, to provide a check on the results and conclusions. Using "un-deformed" circumferences of the body measured under only atmospheric pressure, around $40 \%$ of the leg would be within $5 \mathrm{kPa}$ of the desired $30 \mathrm{kPa}$, which should be considered encouraging, since when the body is placed under MCP, it deforms to balance out the pressures. The analysis suggests that MCP is a viable alternative to gas-pressurized spacesuits.

A number of experiments were undertaken to understand why the TekScan sensors used to measure pressure produced by the neoprene bands were reading lower than expected values. It is clear that the TekScan sensor reads the accurate pressures when pressed against a particular surface only when it is calibrated on the same surface. "Soft-to-hard" testing leads to overly high measurements, since the sensor is expecting some surface deformation, but instead receives a strong force. In "hard-tosoft" testing, this hard surface is assumed but the deformation causes readings to be too low. These results indicate that the cause of the pressure variation is indeed with the TekScan system. Additional studies indicate that the TekScan sensor records higher pressures under normal atmospheric pressure than at $-225 \mathrm{~mm} \mathrm{Hg}$ underpressure (relative to atmospheric pressure).

The human experimental tests were conducted at a higher pressure $(-225 \mathrm{~mm} \mathrm{Hg})$ for a longer duration ( 1 hour) with more of the body covered (the right leg from ankle to upper thigh) than had ever been conducted previously. In order to accurately pressurize the human body via MCP, the measurements of the human body must be taken when it is under MCP compression, otherwise the subject will experience underpressure. The neoprene bands appear to be effectively protecting the leg, with the exception of the concavities around the knee, from the $-225 \mathrm{~mm} \mathrm{Hg}$ underpressure in the chamber. One test with a knee brace used to fill these concavities with material to allow the neoprene bands to pressurize this area of the body more effectively was successful, but should not be considered conclusive. The seal used in these experiments also contributes the ability to arrach MCP limbs to a pressurized torso for further testing. 


\subsection{Future work}

In order to further understand MCP and its viability as a replacement design for the gas-filled spacesuits currently used on all human spaceflight missions, significant work must be accomplished. This section is a list of some of the most critical activities.

1. Research the pressure differential that humans can withstand in a low pressure environment, as the $1.6 \mathrm{kPa}$ value the team currently uses as a goal is considered conservative.

2. Understand how much compression the human body experiences under varying levels of mechanical counterpressure, and how much it differs between individuals. Is the relationship linear with varying levels of MCP?

3. Conduct further theoretical modeling using data from legs experiencing MCP to provide more accurate results.

4. Produce a full leg prototype that is a closer approximation to the future BioSuit design, rather than using the wrapping procedure which inherently produces some discomfort.

5. Improve knee padding to eliminate edema.

6. Develop a permeable elastic material with anisotropic elastic properties in order to test knee bending.

7. Improve the hybrid boot to a realistic operational design.

8. Find or develop a more accurate pressure measuring system to replace the TekScan system to accurately record pressure on the human body. Therefore, the edema experienced by the subjects can be compared to a map of measured underpressure or overpressure on the human body. 


\section{Appendix A: Derivation of Young's Modulus equations}

This appendix section details the derivation for the equations used to find the percent strain needed for a given Young's modulus and starting thickness in Chapter 2.

From the definition of stress:

where,

$$
\sigma=\frac{F}{A}
$$

$\sigma=$ stress, $\mathrm{Pa}$

$\mathrm{F}=$ force in tension, $\mathrm{N}$

$\mathrm{A}=$ cross-sectional area, $\mathrm{m}^{2}$

Since the force is circumferential, the cross-sectional area of the material is:

$$
\text { where, } \quad \begin{aligned}
& \mathrm{w}=\text { width of the material, } \mathrm{m} \\
& \mathrm{t}=\text { thickness of the material, } \mathrm{m}
\end{aligned}
$$

Using the law of thin-walled cylindrical pressure vessels, the stress in the material can also be determined by:

where,

$$
\sigma=\frac{P \times r}{t}
$$

$$
\begin{aligned}
& \mathrm{P}=\text { pressure }, \mathrm{Pa} \\
& \mathrm{r}=\text { radius of body, } \mathrm{m}
\end{aligned}
$$

Substituting Eq. 5 into Eq. 4, and the setting the stresses in Eq. 4 and Eq. 6 equal to each other yields:

$$
F=P \times r \times w
$$

Therefore, the tension necessary to define the proper pressure depends only on the radius of the body part the elastic is pressurizing, and the width of the material. It makes sense to rewrite this equation slightly, defining a force/width, in order to normalize the force needed to create the certain level of MCP.

Therefore, the tension necessary in the rubber (per $\mathrm{cm}$ of width) to produce the proper pressure (30 $\mathrm{kPa})$ on a $2.18 \mathrm{~cm}$ radius wrist $\left(5^{\text {th }}\right.$ percentile female) is $6.54 \mathrm{~N} / \mathrm{cm}$.

The tension necessary in the rubber (per $\mathrm{cm}$ of width) to produce the proper pressure $(30 \mathrm{kPa})$ on a $10.73 \mathrm{~cm}$ radius thigh $\left(95^{\text {th }}\right.$ percentile male) is $32.19 \mathrm{~N} / \mathrm{cm}$. 
Using Hooke's Law:

$\begin{array}{ll} & \sigma=E \times \varepsilon \\ \text { where, } & \mathrm{E}=\text { Young's Modulus, } \mathrm{Pa} \\ \varepsilon=\text { strain, unitless } & \text { (Equation 7) }\end{array}$

Setting Eq. 6 and Eq. 7 equal to each other and some simple arithmetic yields:

$$
\varepsilon=\frac{P \times r}{E \times t}
$$

To determine the percent elongation, the following equation is then used:

where,

$$
\% \text { Elong }=\varepsilon \times 100
$$

Combining Eq. 8 and Eq. 9 readily yields an equation for percent elongation.

These calculations assume only one layer of material, but if another layer is added, the pressures would add accordingly. Therefore, adding layers would reduce the pressure required from each band, and reduce the percent elongation required in each band. This has the same effect as assuming 1 layer, but with the thickness based on the addition of all the layers together. In other words, the same strain values are calculated from 1 layer with a $2 \mathrm{~cm}$ thickness, and 2 layers each with a $1 \mathrm{~cm}$ thickness, if all the layers have the same Young's Modulus.

The following equations were not used as part of this thesis, but could be useful for a more detailed analysis of a particular material.

In order to identify the ideal material properties, definition of poisson's ratio must be stated as well:

where,

$$
v=-\frac{\varepsilon_{t}}{\varepsilon_{l}}
$$

$$
\begin{aligned}
& v=\text { poisson's ratio, dimensionless } \\
& \varepsilon_{\mathrm{t}}=\text { transverse strain (thickness), dimensionless } \\
& \varepsilon_{\mathrm{l}}=\text { longitudinal strain, dimensionless }
\end{aligned}
$$

With a slight reorganization of the variables in Eq. 8, the following equation is found:

$$
\varepsilon_{l} \times t_{f}=\frac{P \times r}{E}
$$

where,

$$
\mathrm{t}_{\mathrm{f}}=\text { final thickness, } \mathrm{m}
$$

Using the definition of strain,

where,

$$
\varepsilon=\frac{x_{f}-x_{o}}{x_{o}}
$$

$$
\mathrm{x}_{0}=\text { initial dimension, } \mathrm{m}
$$


and substituting $\mathrm{t}_{\mathrm{f}}$ from Eq. 10 into Eq. 2 leaves a quadratic equation for $\varepsilon_{1}$. After completing the square, the following equation is found:

$$
\varepsilon_{l}=\frac{1}{2 v} \pm \sqrt{\frac{-P \times r}{E \times t_{o} \times v}+\frac{1}{4 \times v^{2}}}
$$

To solve for the final thickness, $\varepsilon_{1}$ can be substituted back into Eq. 10. Then Eq. 2 can be used to check the result to make sure it is accurate. Note that one combination of parameters can lead to 2 possible solutions. 


\section{Appendix B: Producing pressure over the knee with longitudinal bands}

When astronauts (or any person) bend their legs, the amount of surface area of the skin on the anterior of the knee increases while the amount of surface area on the posterior decreases. This situation is not a difficulty for a gas-filled spacesuit, as the entire volume exposed to the body has the same pressure. However, for a MCP-based design, the material over the knee (or elbow) must react to this change in surface area in order to keep the pressure production constant. One solution to this problem is to have a mechanism that can sense the surface area increase or decrease and extend or contract the elastic material to keep the pressure constant. Such rapid stretching and contracting could be exceedingly difficult and complicated, as well as damaging to the elastic material. Another solution would be to include a small sac of either gas or liquid around the joint. Since the purpose of a MCP design is to break away from this paradigm and its limitations, this method for pressure production is not the first choice.

A third alternative, to be discussed in this section, is the possibility of using longitudinal bands to create pressure on this part of the body, in addition to using circumferential bands. A bent knee (or elbow) provides a radius of curvature, and so the same MCP principles discussed previously apply here as well. Pressure production via this means would not be ideal, since it would cause more work for the astronaut to bend the joint. However, as long as the mobility is still increased from the current gas-filled suit, such a design would be acceptable.

The scenario examined here to identify the maximum torque produced when kneeling, ie the knee at $90^{\circ}$. When the knee bends 90 degrees, it causes approximately a 30\% longitudinal strain and a $10 \%$ circumferential strain [12]. This combination yields a change in pressure of $-30 \%$ if the force is assumed to be constant, based on Equation 1. Therefore, if the initial pressure over the knee is 30 $\mathrm{kPa}$, the new pressure is $21 \mathrm{kPa}$. The blue line in the following figure traces the shape of the kneecap when bent. This distance is much smaller when the leg is straight, hence the change in surface area. The reverse is true for the posterior of the knee, leading to an increase in pressure when the knee is bent. The red lines indicate the longitudinal radius of curvature, with the approximate center of this "circle" is shown by the green diamond. 


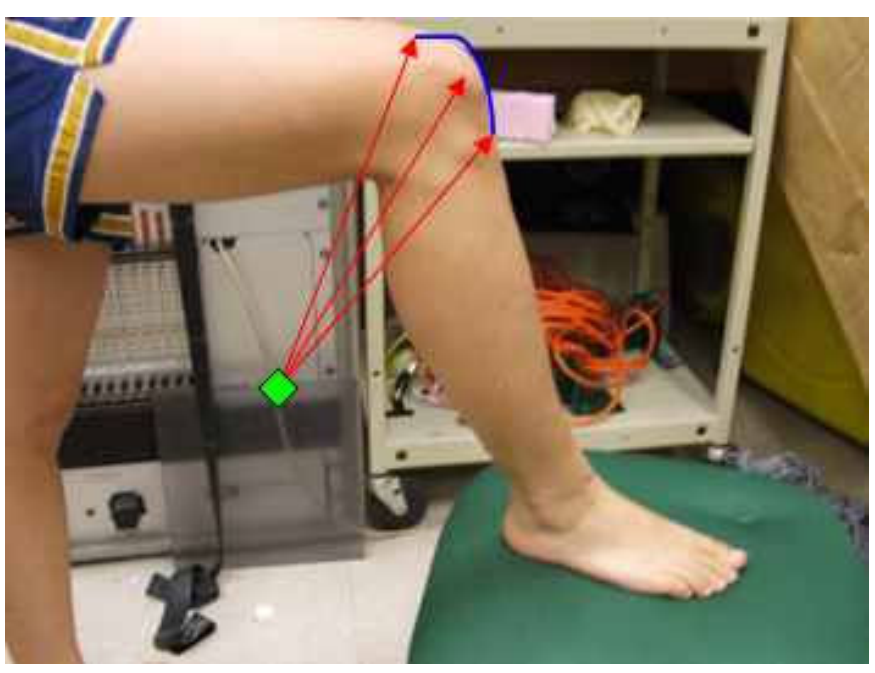

Figure 90: Explanation of longitudinal cross-section and change in surface area of skin

Defining torque as:

where,

$$
\begin{gathered}
\tau=r \times F \\
\tau=\text { torque, } \mathrm{N}-\mathrm{m}
\end{gathered}
$$

Then substitution of Eq. 1 into Eq.13 yields:

$$
\tau=P \times r^{2} \times w
$$

The width is assumed to be the cross-sectional diameter of the knee, $12 \mathrm{~cm}$ and can be easily calculated given that the circumference of the average knee is $38 \mathrm{~cm}$. The average longitudinal radius of curvature is $12.73 \mathrm{~cm}$ at $90^{\circ}$ [4], so the torque necessary to offset this $9 \mathrm{kPa}$ pressure loss is $17.5 \mathrm{~N}-\mathrm{m}$.

This is somewhat greater than the $\mathbf{1 4 . 6} \mathbf{~ N - m}$ torque currently caused to move the knee in the EMU to $72^{\circ}$ [4], although it should be noted that more torque would be expected for the larger angle. The small difference between the values reveals that pressure could potentially be created in this manner without significant increase in movement difficulty compared to the current spacesuit.

Using such torques to create pressure would impair the mobility of the astronauts and drive up their metabolic rates. A trade must be made between the loss of pressure and the mobility needs to determine what, if any, pressure should be produced using longitudinal fibers in the elastic material. 


\section{Appendix C: Full display of Young's Modulus ranges}

The figure below shows the full range of data described in 2.8.3: Fabric Requirements.

Young's modulus ranges

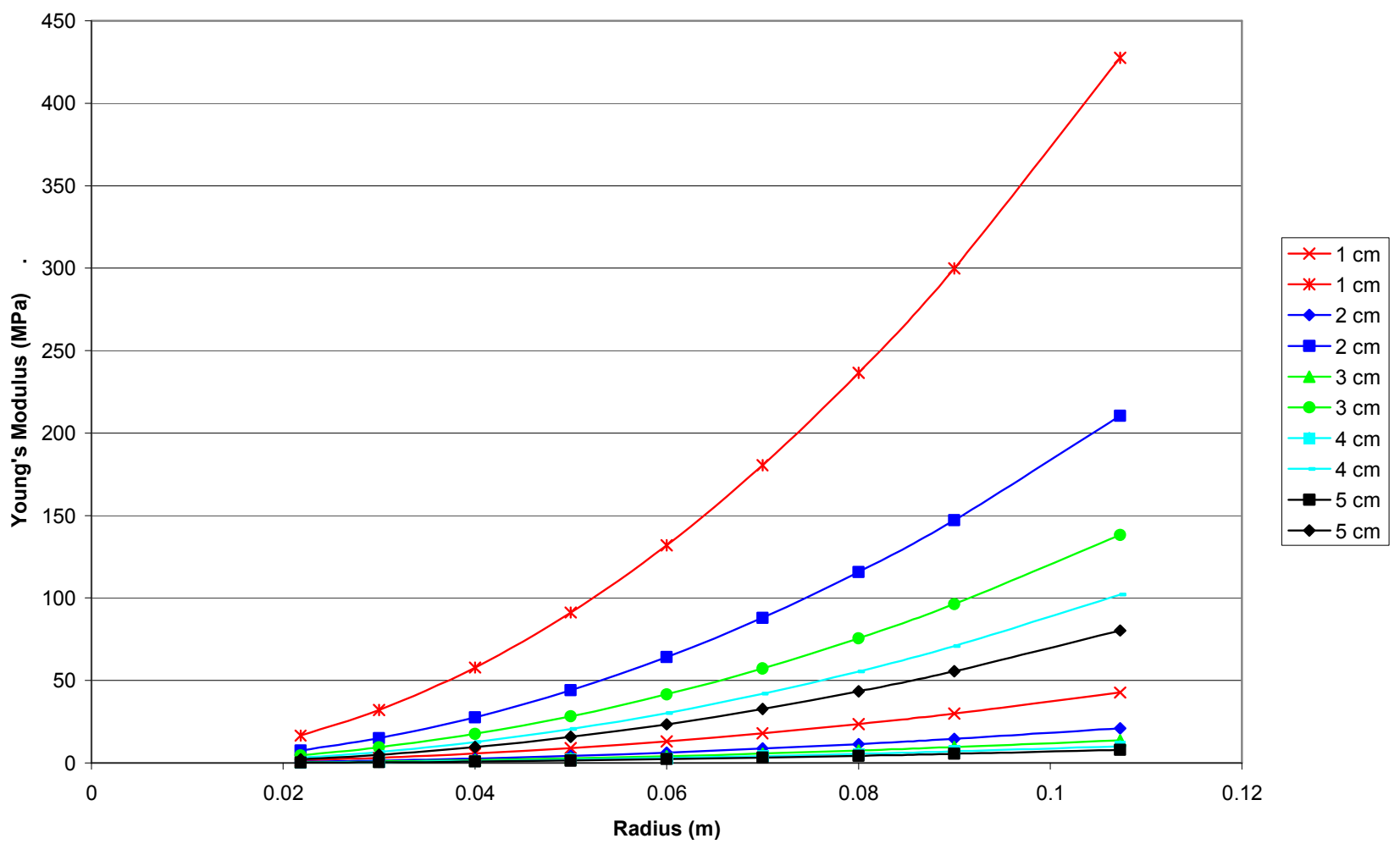

Figure 91: Full display of Young's Modulus ranges

The following section details all of the data points used to create the proceeding figure. 
Table 36: Table of values for Young's Modulus ranges

\begin{tabular}{|c|c|c|c|c|c|}
\hline $\begin{array}{c}\text { Radius } \\
\text { (m) }\end{array}$ & $\begin{array}{c}\text { Distance SMP must } \\
\text { close (m) }\end{array}$ & Strain & $\begin{array}{l}\text { Et product } \\
(\mathrm{N} / \mathrm{m})\end{array}$ & $\begin{array}{l}\text { E low }(t=5 \\
\text { mm) MPa }\end{array}$ & $\begin{array}{c}E \max (t=0.5 \\
\mathrm{mm}) \mathrm{MPa}\end{array}$ \\
\hline 0.0218 & 0.01 & 0.08 & 8304.06 & 1.66 & 16.61 \\
\hline 0.03 & 0.01 & 0.06 & 16064.60 & 3.21 & 32.13 \\
\hline 0.04 & 0.01 & 0.04 & 28959.29 & 5.79 & 57.92 \\
\hline 0.05 & 0.01 & 0.03 & 45623.89 & 9.12 & 91.25 \\
\hline 0.06 & 0.01 & 0.03 & 66058.40 & 13.21 & 132.12 \\
\hline 0.07 & 0.01 & 0.02 & 90262.82 & 18.05 & 180.53 \\
\hline 0.08 & 0.01 & 0.02 & 118237.16 & 23.65 & 236.47 \\
\hline 0.09 & 0.01 & 0.02 & 149981.40 & 30.00 & 299.96 \\
\hline 0.1073 & 0.01 & 0.02 & 213801.40 & 42.76 & 427.60 \\
\hline 0.0218 & 0.02 & 0.17 & 3825.03 & 0.77 & 7.65 \\
\hline 0.03 & 0.02 & 0.12 & 7582.30 & 1.52 & 15.16 \\
\hline 0.04 & 0.02 & 0.09 & 13879.64 & 2.78 & 27.76 \\
\hline 0.05 & 0.02 & 0.07 & 22061.94 & 4.41 & 44.12 \\
\hline 0.06 & 0.02 & 0.06 & 32129.20 & 6.43 & 64.26 \\
\hline 0.07 & 0.02 & 0.05 & 44081.41 & 8.82 & 88.16 \\
\hline 0.08 & 0.02 & 0.04 & 57918.58 & 11.58 & 115.84 \\
\hline 0.09 & 0.02 & 0.04 & 73640.70 & 14.73 & 147.28 \\
\hline 0.1073 & 0.02 & 0.03 & 105291.20 & 21.06 & 210.58 \\
\hline 0.0218 & 0.03 & 0.28 & 2332.02 & 0.47 & 4.66 \\
\hline 0.03 & 0.03 & 0.19 & 4754.87 & 0.95 & 9.51 \\
\hline 0.04 & 0.03 & 0.14 & 8853.10 & 1.77 & 17.71 \\
\hline 0.05 & 0.03 & 0.11 & 14207.96 & 2.84 & 28.42 \\
\hline 0.06 & 0.03 & 0.09 & 20819.47 & 4.16 & 41.64 \\
\hline 0.07 & 0.03 & 0.07 & 28687.61 & 5.74 & 57.38 \\
\hline 0.08 & 0.03 & 0.06 & 37812.39 & 7.56 & 75.62 \\
\hline 0.09 & 0.03 & 0.06 & 48193.80 & 9.64 & 96.39 \\
\hline 0.1073 & 0.03 & 0.05 & 69121.13 & 13.82 & 138.24 \\
\hline 0.0218 & 0.04 & 0.41 & 1585.52 & 0.32 & 3.17 \\
\hline 0.03 & 0.04 & 0.27 & 3341.15 & 0.67 & 6.68 \\
\hline 0.04 & 0.04 & 0.19 & 6339.82 & 1.27 & 12.68 \\
\hline 0.05 & 0.04 & 0.15 & 10280.97 & 2.06 & 20.56 \\
\hline 0.06 & 0.04 & 0.12 & 15164.60 & 3.03 & 30.33 \\
\hline 0.07 & 0.04 & 0.10 & 20990.71 & 4.20 & 41.98 \\
\hline 0.08 & 0.04 & 0.09 & 27759.29 & 5.55 & 55.52 \\
\hline 0.09 & 0.04 & 0.08 & 35470.35 & 7.09 & 70.94 \\
\hline 0.1073 & 0.04 & 0.06 & 51036.10 & 10.21 & 102.07 \\
\hline 0.0218 & 0.05 & 0.57 & 1137.61 & 0.23 & 2.28 \\
\hline 0.03 & 0.05 & 0.36 & 2492.92 & 0.50 & 4.99 \\
\hline 0.04 & 0.05 & 0.25 & 4831.86 & 0.97 & 9.66 \\
\hline 0.05 & 0.05 & 0.19 & 7924.78 & 1.58 & 15.85 \\
\hline 0.06 & 0.05 & 0.15 & 11771.68 & 2.35 & 23.54 \\
\hline 0.07 & 0.05 & 0.13 & 16372.56 & 3.27 & 32.75 \\
\hline 0.08 & 0.05 & 0.11 & 21727.43 & 4.35 & 43.45 \\
\hline 0.09 & 0.05 & 0.10 & 27836.28 & 5.57 & 55.67 \\
\hline 0.1073 & 0.05 & 0.08 & 40185.08 & 8.04 & 80.37 \\
\hline
\end{tabular}




\section{Appendix D: Matlab code for Young's Modulus theoretical calculations}

$\%\{$

Author: Dan Judnick

Purpose: This program is designed to recognize the ideal material

properties to create MCP.

Using the law of thin-pressure vessels, the given eq' $n$ is:

$\mathrm{P}=\mathrm{E}^{*} \mathrm{e}^{*} \mathrm{t} / \mathrm{r}$, where:

$\mathrm{P}=$ Pressure, $\mathrm{Pa}$ (here specified as $30^{*} 10^{\wedge} 3 \mathrm{~Pa}=30 \mathrm{kPa}$ )

$\mathrm{E}=$ Young's Modulus of Material, $\mathrm{Pa}$

$\mathrm{e}=$ strain, dimensionless

$\mathrm{t}=$ thickness of the material, $\mathrm{m}$ (always less than $5 \mathrm{~mm}$ )

$r=$ radius of the body part, $m$

The radius will range from the radius of a 5th percentile woman's wrist $(2.18 \mathrm{~cm})$ to 95 th percentile male's thigh $(10.73 \mathrm{~cm})$.

Additional midway values of $5 \mathrm{~cm}$ and $7.5 \mathrm{~cm}$ will also be considered.

Note: Anthropomorphic data based on NASA-STD-3000 from year 2000.

First the Young's modulus will be varied for various thicknesses and the radii described above to determine the strain.

Then the strain will be varied over the same thicknesses and radii to determine the Young's Modulus needed.

Date: $2 / 22 / 06$

$\%\}$

clc

clear

rb=[.0218,.03,.04,.05,.06,.07,.08,.09,..1073];

\%Radius of Body in $\mathrm{m}$

Pconst $=30 ; \%$ desired pressure in $\mathrm{kPa}$

$\mathrm{n}=1 ; \%$ a simple counter

tmax $=0.005 ; \% 5 \mathrm{~mm}$, any larger, and too thick- BioSuit requirements

tmin $=0.0005$; \%any smaller, probably too thin (is $0.5 \mathrm{~mm}$ )

for distance $=1: 5 \%$ distance in $\mathrm{cm}$ that the SMP must close

distance $=$ distance $/ 100$; \%back into $\mathrm{m}$

for $n 3=1$ :length(rb) \% loops the body radius 


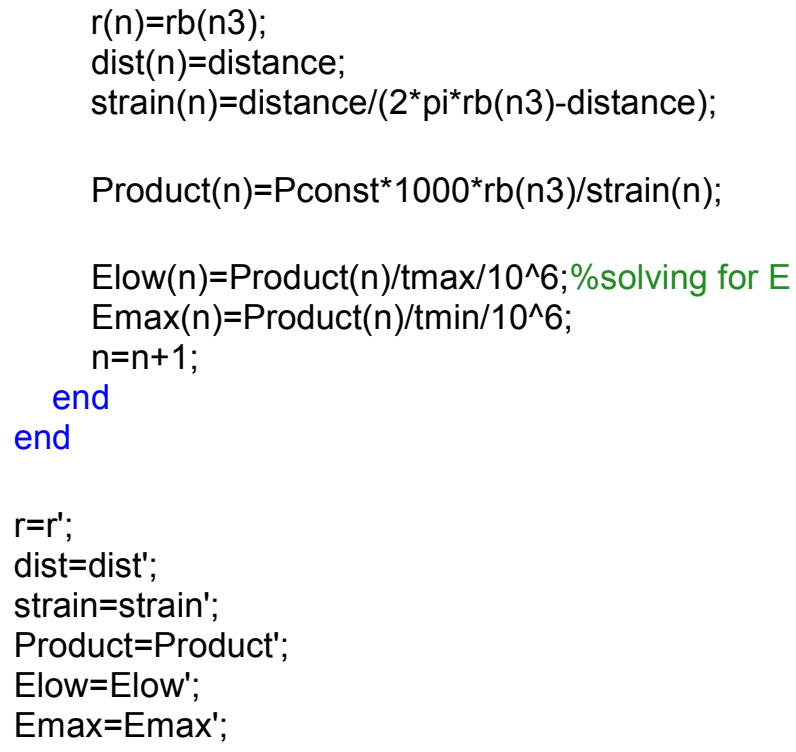




\section{Appendix E: Matlab code for analysis using Wolfrum data}

\subsection{Code for Wolfrum data}

\subsubsection{Neighbor Normal Method code}

\subsubsection{Neighbor Normal Start.m}

$\%$ This file, NeighborNormalStart, simply calls the other two functions, and

$\%$ produces the titles for the graphs

\% Dan Judnick, 3/3/07

clc

clear

close all

for $\operatorname{sub}=1: 6$

[normal_vectors]=NormalVectorsFinal(sub);

[markers,rbodyinit]=NeighborNormal(sub,normal_vectors);

if $\mathrm{sub}==1$

title('Subject 1')

elseif $\mathrm{sub}==2$

title('Subject 2')

elseif $\mathrm{sub}==3$

title('Subject 3')

elseif $s u b==4$

title('Subject 4')

elseif $\mathrm{sub}==5$

title('Subject 5')

elseif $\mathrm{sub}==6$

title('Subject 6')

end

end

\subsubsection{Normal Vectors Final.m}

function [normal_vectors]=NormalVectorsFinal(sub) 
$\%$ This m-file takes mesh data from each of the 6 subjects, and loads it into $\%$ Matlab to determine the perpendicular vector at that point.

$\%$ The sole input is the number of the subject. To run the code properly, $\%$ the desired files must be in the same folder as this $\mathrm{m}$-file. Please note $\%$ that these are not the proper file names to be loaded, as they had to be $\%$ changed to remove the name of the subjects.

$\%$ I didn't actually use the data from the bent knee at 90 degrees, but left $\%$ it in case a comparison of the bent leg was desired. Only small changes $\%$ would be necessary to do the analysis.

$\%$ This code was not originally written by this author, but rather by Nina $\%$ Wolfrum. A small error was discovered, and fixed as noted below. For a $\%$ better understanding of this code, please see her thesis.

\%Dan Judnick, 3/3/07

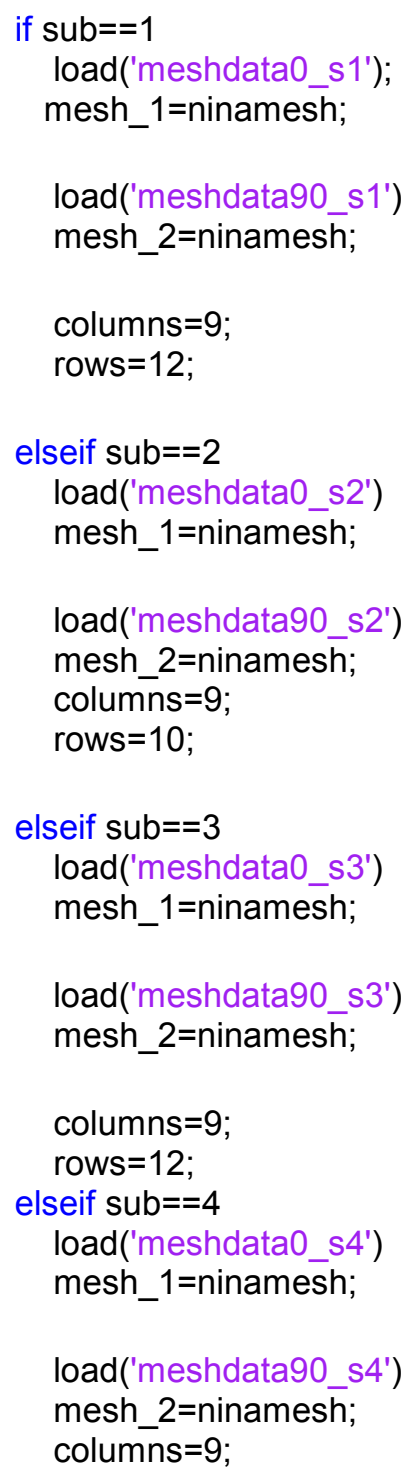




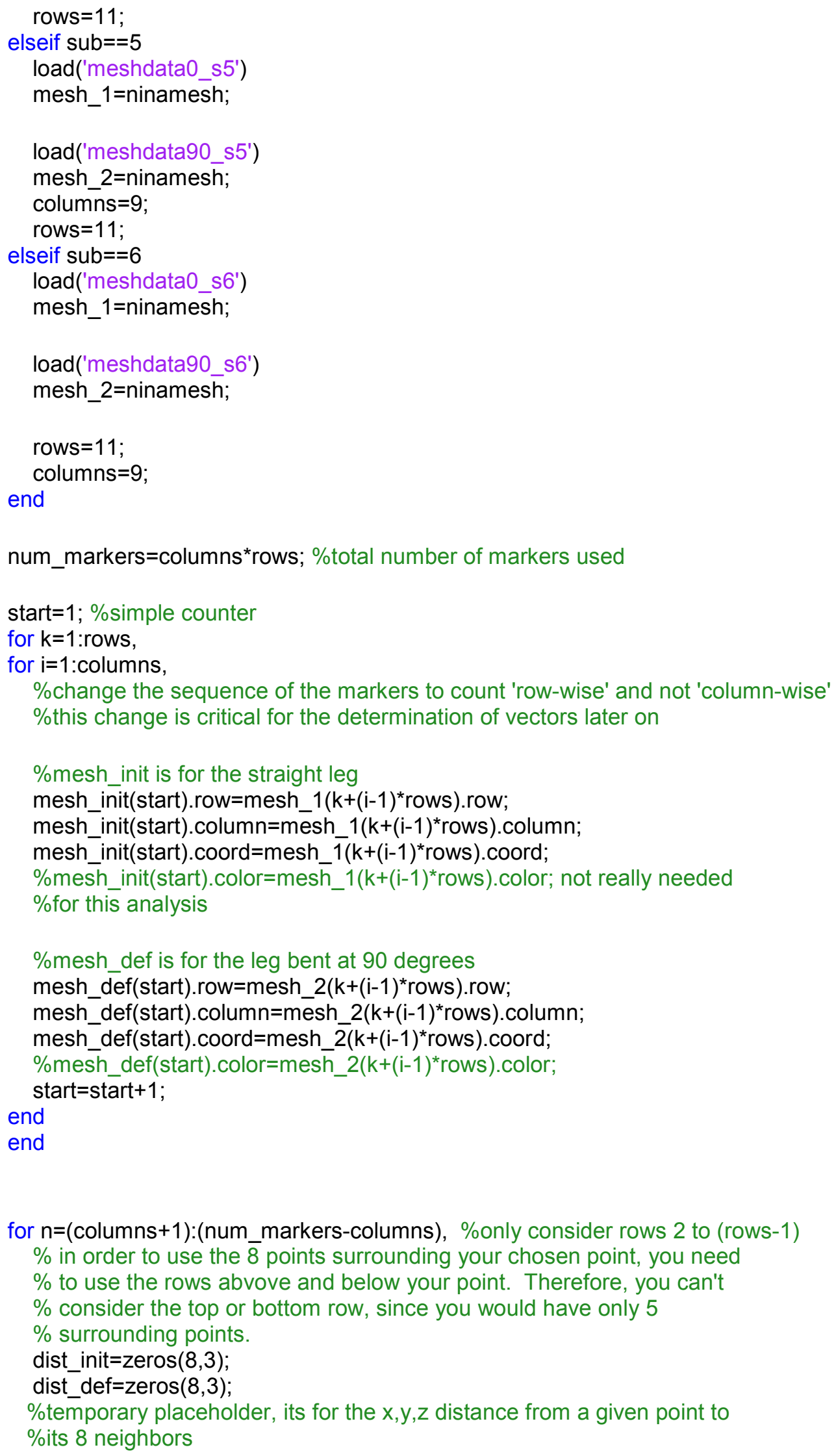

for $n=($ columns +1$)$ :(num_markers-columns), \%only consider rows 2 to (rows- 1 )

$\%$ in order to use the 8 points surrounding your chosen point, you need

$\%$ to use the rows abvove and below your point. Therefore, you can't

$\%$ consider the top or bottom row, since you would have only 5

$\%$ surrounding points.

dist_init=zeros $(8,3)$;

dist_def=zeros $(8,3)$;

\%temporary placeholder, its for the $x, y, z$ distance from a given point to

\%its 8 neighbors 


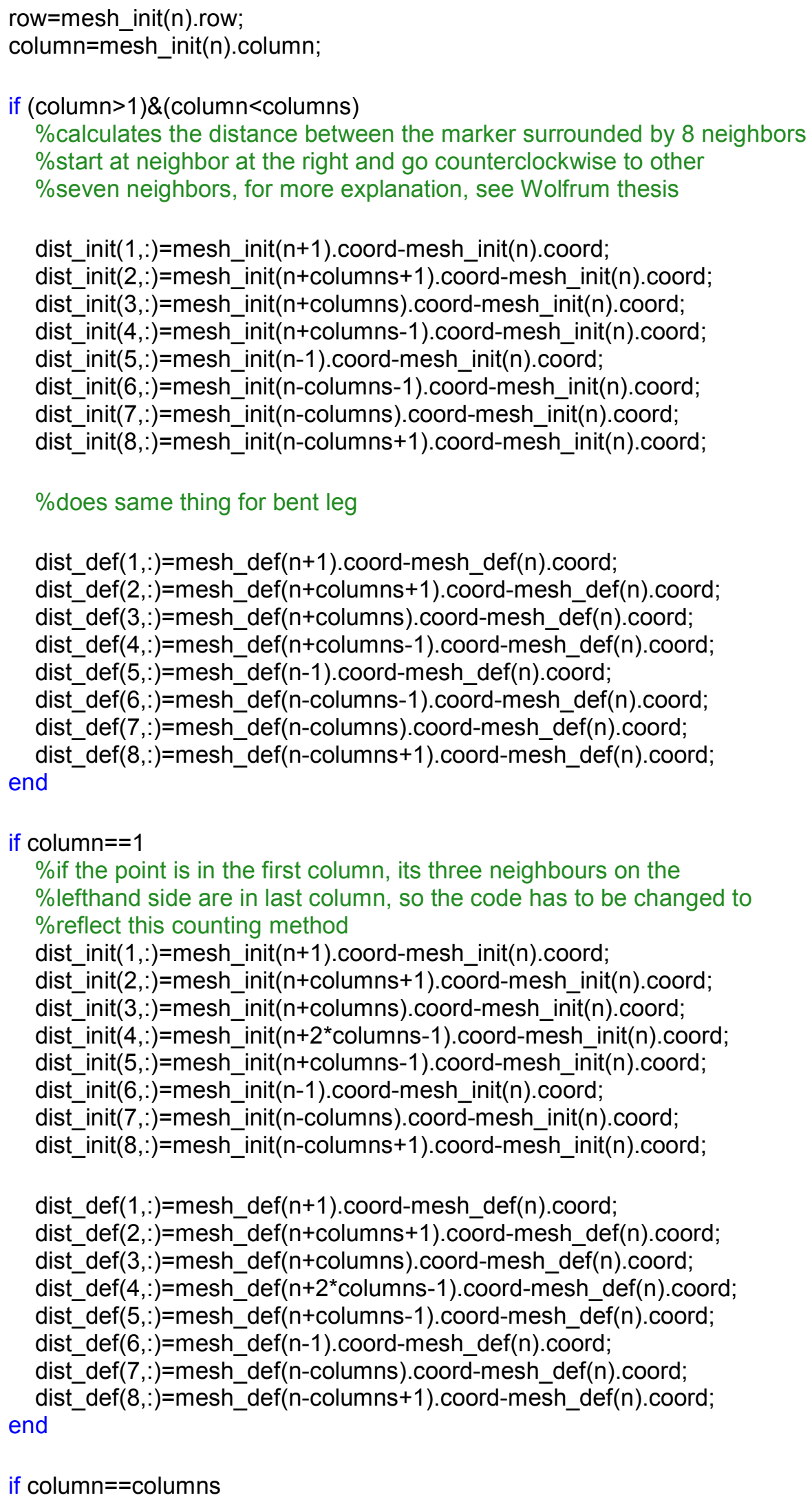


$\%$ here the markers on the righthand side are in first column, so

$\%$ again some slight changes must be made to get the appropriate

$\%$ neighbors

dist_init(1,::)=mesh_init(n-columns+1).coord-mesh_init(n).coord;

dist_init(2,:)=mesh_init(n+1).coord-mesh_init(n).coord;

dist_init(3,:)=mesh_init $(n+$ columns).coord-mesh_init(n).coord;

dist_init(4,:)=mesh_init(n+columns-1).coord-mesh_init(n).coord;

dist_init(5,:)=mesh_init(n-1).coord-mesh_init(n).coord;

dist_init(6,:)=mesh_init(n-columns-1).coord-mesh_init(n).coord;

dist_init $(7,:)=$ mesh_init(n-columns).coord-mesh_init(n).coord;

dist_init(8,:)=mesh_init(n-2*columns+1).coord-mesh_init(n).coord;

dist_def(1,:)=mesh_def(n-(columns-1)).coord-mesh_def(n).coord;

dist_def(2,:)=mesh_def(n+1).coord-mesh_def(n).coord;

dist_def(3,:)=mesh_def(n+columns).coord-mesh_def(n).coord;

dist_def(4,:)=mesh_def(n+columns-1).coord-mesh_def(n).coord;

dist_def(5,:)=mesh_def(n-1).coord-mesh_def(n).coord;

dist_def $(6,:)=$ mesh_def(n-columns-1).coord-mesh_def(n).coord;

dist_def $(7,:)=$ mesh_def(n-columns).coord-mesh_def(n).coord; end

dist_def(8,:)=mesh_def(n-2*columns+1).coord-mesh_def(n).coord;

\%calculating eight normal vectors between pairs of neighboring vectors

normals=zeros $(8,3) ; \%$ placeholder

for $\mathrm{i}=1: 7$,

\%ORIGINAL CODE, but normal vectors in wrong direction (point inwards)!!!!!!!!!!!!!!!

$\%$ normals $(\mathrm{i},: \mathrm{:})=\operatorname{cross}($ dist_init(i,:), dist_init(i+1,::))/norm(cross(dist_init(i,::),dist_init(i+1,::)));

$\%$ normals2 $(\mathrm{i},:)=\operatorname{cross}\left(\operatorname{dist} \_\operatorname{def}(\mathrm{i},:), \operatorname{dist} \_\operatorname{def}(\mathrm{i}+1,:)\right) / \operatorname{norm}\left(\operatorname{cross}\left(\operatorname{dist} \_\operatorname{def}(\mathrm{i},:), \operatorname{dist} \_\operatorname{def}(\mathrm{i}+1,:)\right)\right)$;

\%Correct code, my edits (the following 2 lines)

normals( $(\mathrm{i},:)=\operatorname{cross}(\operatorname{dist} i n i t(i+1,:)$, dist_init $(\mathrm{i},:)) /$ norm(cross(dist_init(i+1,::),dist_init(i,:))); end

normals2 $(\mathrm{i},:)=\operatorname{cross}\left(\operatorname{dist} \_\operatorname{def}(\mathrm{i}+1,:)\right.$, dist_def(i,:))/norm(cross(dist_def(i+1,:), dist_def(i,:)));

\%AGAIN ORIGINAL CODE, but is incorrect

$\%$ normals $(8,:)=\operatorname{cross}($ dist_init $(8,:)$, dist_init $(1,:)) /$ norm $(\operatorname{cross}($ dist_init( $(8,:)$, dist_init(1,::)));

$\%$ normals2 $(8,:)=\operatorname{cross}\left(\operatorname{dist} \_\operatorname{def}(8,:), \operatorname{dist}\right.$ def $\left.(1,:)\right) / \operatorname{norm}(\operatorname{cross}(\operatorname{dist}$ def $(8,:)$, dist_def $(1,:)))$;

normals $(8,:)=\operatorname{cross}($ dist_init(1,:), dist_init( $8,:)) /$ norm(cross(dist_init(1,::),dist_init( $(8,:)))$;

normals2 $(8,:)=\operatorname{cross}\left(\operatorname{dist} \_\operatorname{def}(1,:), \operatorname{dist} \_\operatorname{def}(8,:)\right) / \operatorname{norm}\left(\operatorname{cross}\left(\operatorname{dist} \_d e f(1,:), \operatorname{dist} \_d e f(8,:)\right)\right)$;

$\%$ calculating average perpendicular vector for one tangent plane to the human body

n_total=[0 0 0 0$]$

n_total2=[ [l 0 0 $]$;

for $i=1: 8$;

n_total=n_total+normals(i,:);

n_total2=n_total2+normals2(i,:);

end

normal_vectors(n).init=n_total/norm(n_total); \% finding the normal vector

mag=sqrt(normal_vectors $(n)$.init(1)^2+normal_vectors(n).init(2)^2+normal_vectors(n).init(3)^2); \%is 1 !

$\%$ this is just a check to make sure everything has been normalized

normal_vectors(n).def=n_total2/norm(n_total2);

mag=sqrt(normal_vectors(n).def(1)^2+normal_vectors(n).def(2)^2+normal_vectors(n).def(3)^2); \% is 1 ! 
end

\subsubsection{Neighbor Normal.m}

function [subject,rbodyinit]=NeighborNormal(sub,normal_vectors)

$\%$ This function does the actual calculations of the local radius of

$\%$ curvature, with the number of the subject and the normals as inputs.

$\%$ The outputs includes the graphs of all the subjects leg, as well as the $\%$ display of each cross-section for a chosen subject, with the normals \%included.

$\%$ Note again that these are not the proper file names, as they had to be $\%$ changed to protect the identities of the subjects.

\%Dan Judnick, 3/3/07

$\mathrm{p}=30000 ; \% \mathrm{~Pa}$, this is the goal pressure

if $\operatorname{sub}==1$

load('strainfield_sub1_init'); \%contains marker data numcols $=9$;

numrows $=12$;

elseif sub $==2$

load('strainfield_sub2_init'); \%contains marker data

numcols $=9$;

numrows $=10$;

elseif $s u b==3$

load('strainfield_sub3_init'); \%contains marker data

numcols $=9$;

numrows $=12$;

elseif sub $==4$

load('strainfield_sub4_init'); \%contains marker data

numcols $=9$;

numrows $=11$;

elseif sub $==5$

load('strainfield_sub5_init'); \%contains marker data

numcols $=9$;

numrows $=11$;

markers

elseif $\mathrm{sub}==6$

load('strainfield_sub6_init'); \%contains marker data

numcols $=9$;

numrows $=11$;

end

\%This loops calculates the circumference of every cross-section by

$\%$ measuring the straight line distance between the points

inc=numcols +1 ;

for $n r=2$ :numrows $-1 \%$ can't use bottom or top rows since need all 8 neighbors for nc $=1$ :numcols 
normal_vectors(inc).init2=normal_vectors(inc).init;

$\%$ silly line of code above, but was trying a number of different

$\%$ options, and this was when I discovered the mistake in the WOlfrum

$\%$ code

\%convert from structure to a vector for the sake of simplicity

$\mathrm{x} 1(\mathrm{nc})=$ markers $(\mathrm{inc}) \cdot \operatorname{coord}(1)$;

$\mathrm{y} 1$ (nc) $=$ markers $($ inc $) \cdot \operatorname{coord}(2)$;

$\mathrm{z} 1(\mathrm{nc})=$ markers $(\mathrm{inc}) \cdot \operatorname{coord}(3)$;

if $\mathrm{nc}==1$

circum $=0$; \%initialized values, this is the circumference

sumrad $=0$;

else

sum $=(x 1(n c)-x 1(n c-1))^{\wedge} 2+(y 1(n c)-y 1(n c-1))^{\wedge} 2+(z 1(n c)-z 1(n c-1))^{\wedge} 2$

$\%$ distance to next point

\%sumcheck=norm (markers(inc).coord-markers(inc-1).coord)

circum=circum +sqrt(sum);

delta_radians=acos(dot(normal_vectors(inc).init2,normal_vectors(inc-1).init2));

radius_curvi(inc) $=$ sqrt(sum)/delta_radians;

sumrad=sumrad+delta_radians; \%just a check if we are measuring $2^{*}$ pi radians

$\%$ RCurvfinal(nr,nc)=radius_curv(inc) $/ 1000$;

end

$\%$ special case, since next point is actually the first point in

$\%$ that cross-section

if $\mathrm{nc}==$ numcols

sum $=(x 1(\mathrm{nc})-\mathrm{x} 1(1))^{\wedge} 2+(\mathrm{y} 1(\mathrm{nc})-\mathrm{y} 1(1))^{\wedge} 2+(\mathrm{z} 1(\mathrm{nc})-\mathrm{z} 1(1))^{\wedge} 2$

circum $=$ circum + sqrt(sum);

totalcircum $(\mathrm{nr})=$ circum;

delta_radians=acos(dot(normal_vectors(inc).init2,normal_vectors(inc-8).init2));

radius_curvi(inc-8)=sqrt(sum)/delta_radians;

product=cross(normal_vectors(inc).init2,normal_vectors(inc-8).init2);

delta_radians $2=\operatorname{asin}($ norm $($ product $))$;

sumrad=sumrad+delta_radians;

radequal2pi=sumrad $/ 2 /$ pi; $\% \% \% \% \%$ CHECK RADIANS HERE

if abs(delta_radians-delta_radians2) $>0.01$

difference=delta_radians-delta_radians2; \%CHECK RADIANS CALC

$\%$ again making sure getting $2^{*}$ pi radians, with 2 different

$\%$ methods

end

end

inc=inc +1 ;

end

end

\%this loop calculates the local radius of curvature, as well as the

$\%$ pressure

inc2=numcols +1 ; 


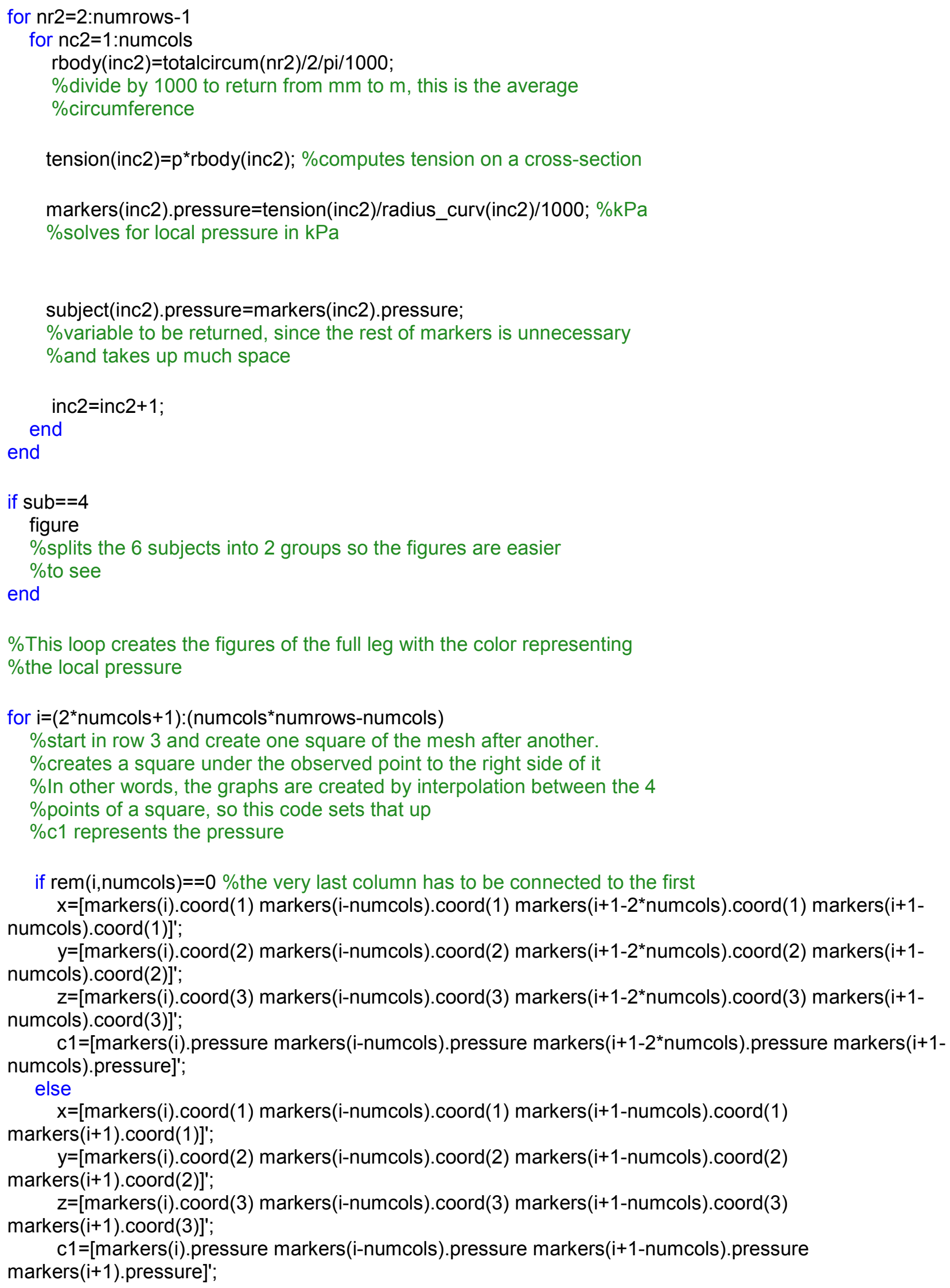




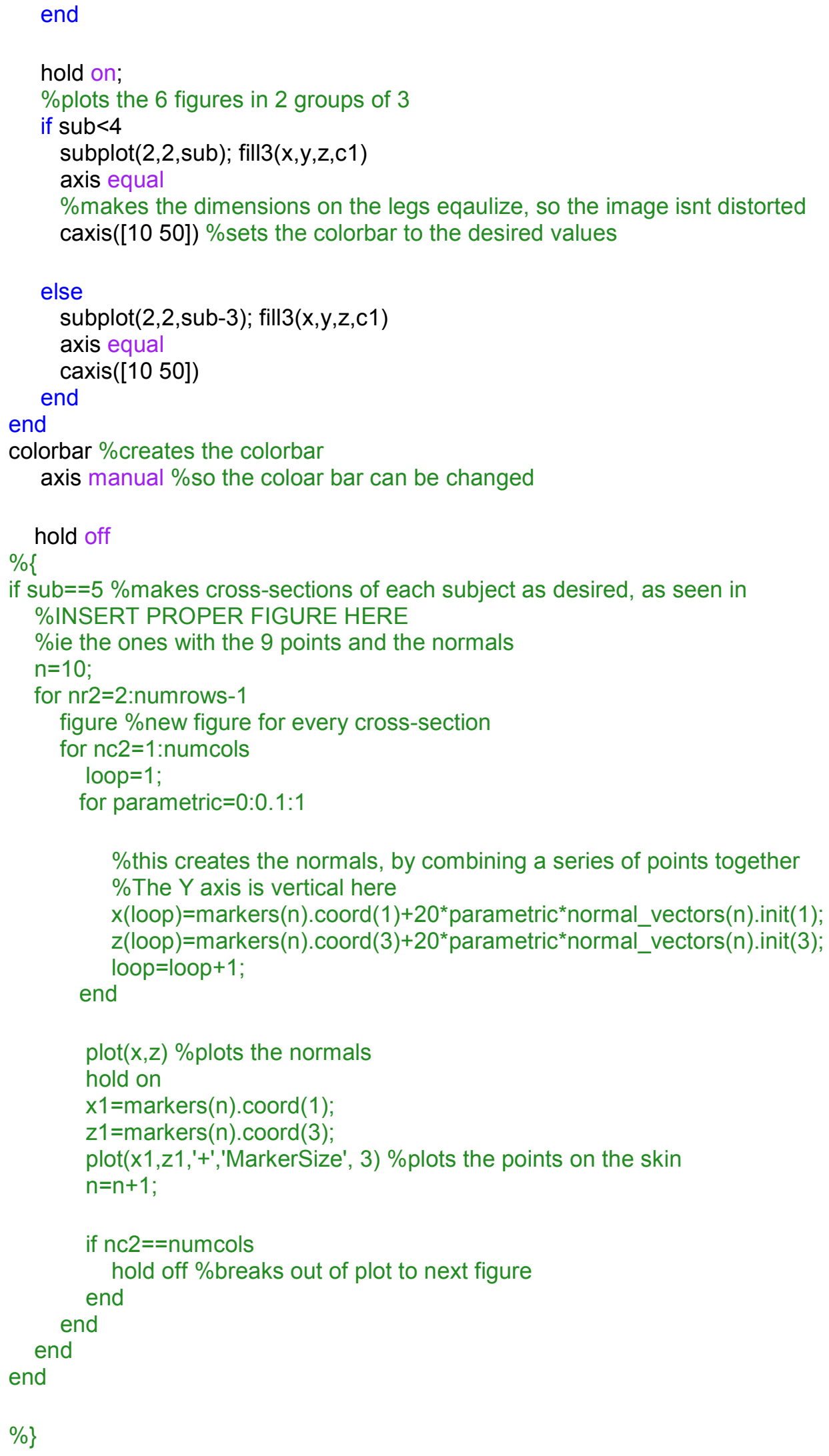




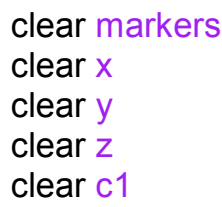

\subsubsection{Point Circular Method}

\subsubsection{Circle Start.m}

$\%$ This file, circleStart, simply calls the other function, and $\%$ produces the titles for the graphs. It can also output some of the $\%$ pressure values to excel, if so desired.

\% Dan Judnick, 3/3/07

clc

clear

close all

for $\operatorname{sub}=1: 6$

[markers,rbodyinit]=LEGgraph0_3pt_circle(sub);

if $\mathrm{sub}==1$ title('Subject 1')

elseif $\mathrm{sub}==2$ title('Subject 2')

elseif $\mathrm{sub}==3$ title('Subject 3') elseif $\mathrm{sub}==4$ title('Subject 4')

elseif $\mathrm{sub}==5$ title('Subject 5') elseif $s u b==6$ title('Subject 6') end

end

\subsubsection{LEG graph0 3pt circle.m}

function [markers,rbodyinit]=LEGgraph0_3pt_circle(sub,)

$\%$ This function does the actual calculations of the local radius of $\%$ curvature, with the number of the subject as an input.

$\%$ The outputs includes the graphs of all the subjects leg, as well as the \%display of each cross-section for a chosen subject, with the normals 
\%included.

$\%$ Note again that these are not the proper file names, as they had to be $\%$ changed to protect the identities of the subjects.

\%Dan Judnick, 3/3/07

$p=30000 ; \% \mathrm{~Pa}$, this is the goal pressure

if $\mathrm{sub}==1$

load('strainfield_sub1_init'); \%contains marker data

numcols $=9$;

numrows $=12$;

elseif sub $==2$

load('strainfield_sub2_init'); \%contains marker data

numcols $=9$;

numrows $=10$;

elseif sub $==3$

load('strainfield_sub3_init'); \%contains marker data

numcols $=9$;

numrows $=12$;

elseif $\mathrm{sub}==4$

load('strainfield_sub4_init'); \%contains marker data

numcols $=9$;

numrows $=11$;

elseif sub $==5$

load('strainfield_sub5_init'); \%contains marker data

numcols $=9$;

numrows $=11$;

markers

elseif $\mathrm{sub}==6$

load('strainfield_sub6_init'); \%contains marker data

numcols $=9$;

numrows $=11$;

end

\% This loops calculates the circumference of every cross-section by

$\%$ measuring the straight line distance between the points

inc=numcols +1 ;

for $\mathrm{nr}=2$ :numrows-1 \%can't use bottom or top rows since need all 8 neighbors for $\mathrm{nc}=1$ :numcols

\%convert from structure to a vector for the sake of simplicity

$\mathrm{x} 1$ (nc) $=$ markers (inc) $\cdot \operatorname{coord}(1)$;

$\mathrm{y} 1(\mathrm{nc})=$ markers $(\mathrm{inc}) \cdot \operatorname{coord}(2)$;

$\mathrm{z} 1(\mathrm{nc})=$ markers (inc) $\cdot \operatorname{coord}(3)$;

if $\mathrm{nc}==1$

circum $=0$;

sumrad=0;

else

$\%$ distance to next point

sum $=(x 1(n c)-x 1(n c-1))^{\wedge} 2+(y 1(n c)-y 1(n c-1))^{\wedge} 2+(z 1(n c)-z 1(n c-1))^{\wedge} 2$

circum $=$ circum + sqrt(sum); 


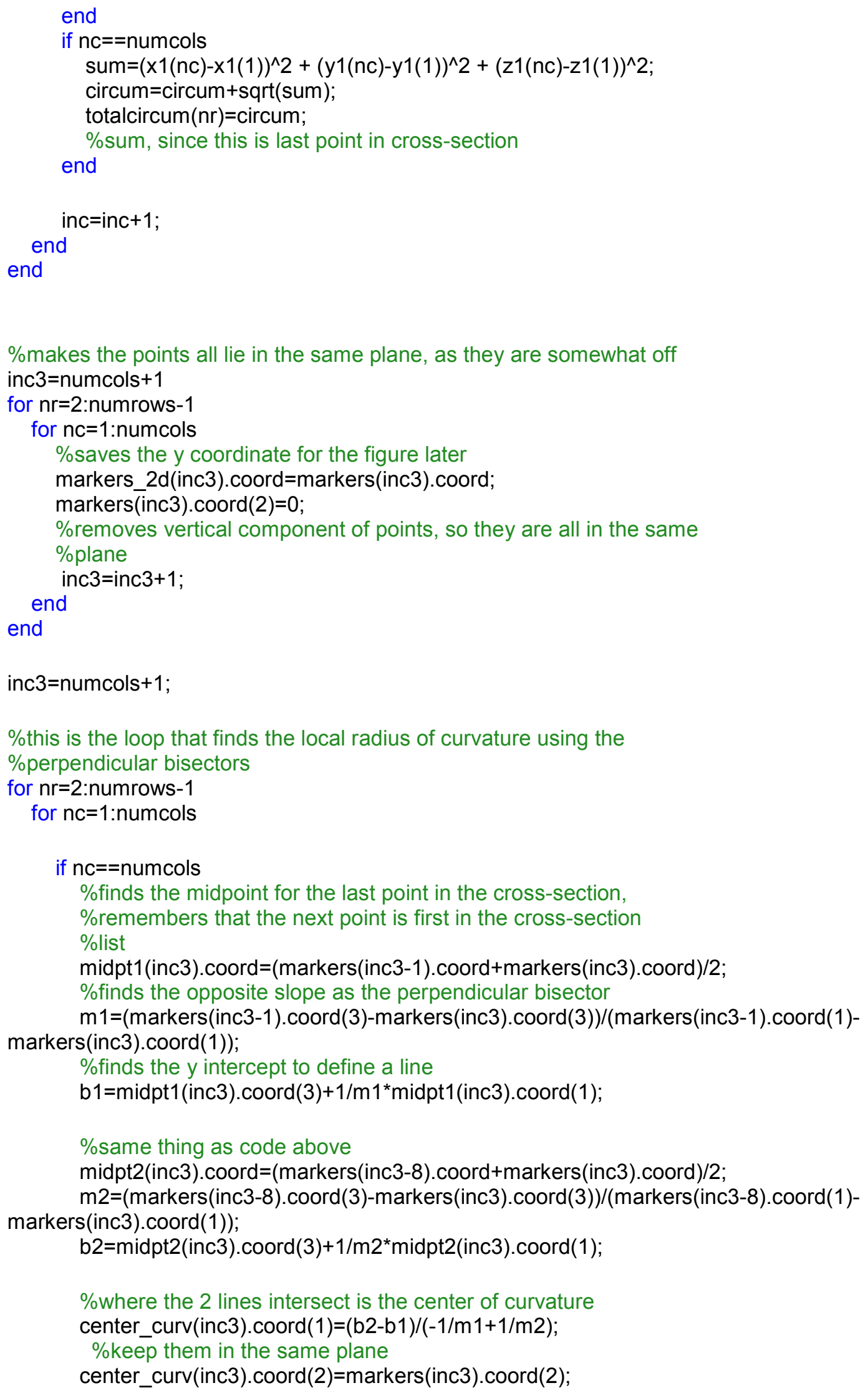


\%finds the z coordinate

center_curv(inc3).coord $(3)=b 2-1 / m 2^{*} x$;

\%Radius of curvature

markers(inc3).radius_curv=norm(markers(inc3).coord-center_curv(inc3).coord);

$\%$ same thing as above, except you have to worry that the previous

$\%$ point is actually the 9th in the cross-section, and they must

\%be connected

elseif $n c==1$

midpt 1 (inc3) . coord $=($ markers (inc3+8). coord+markers(inc3). coord)/2;

$\mathrm{m} 1=($ markers (inc3+8).coord(3)-markers(inc3) $\cdot \operatorname{coord}(3)) /($ markers(inc3+8).coord(1)-

markers(inc3).coord(1));

b1=midpt1 (inc3) $\cdot \operatorname{coord}(3)+1 / \mathrm{m}^{*}{ }^{*} \operatorname{midpt} 1$ (inc3) $\cdot \operatorname{coord}(1)$;

midpt2(inc3).coord $=($ markers (inc3+1).coord +markers(inc3).coord)/2;

$\mathrm{m} 2=($ markers $($ inc3 +1$) \cdot \operatorname{coord}(3)$-markers(inc3).coord(3))/(markers(inc3+1).coord(1)markers(inc3).coord(1));

b2=midpt2(inc3).coord(3)+1/m2*midpt2(inc3) $\cdot \operatorname{coord}(1)$;

center_curv(inc3).coord $(1)=($ b2 $-b 1) /(-1 / m 1+1 / m 2)$;

$\mathrm{x}=(\mathrm{b} 2-\mathrm{b} 1) /(-1 / \mathrm{m} 1+1 / \mathrm{m} 2)$;

center_curv(inc3).coord (2)=markers (inc3).coord(2);

center_curv(inc3).coord $(3)=b 2-1 / \mathrm{m}^{*} \mathrm{x}$;

markers(inc3).radius_curv=norm(markers(inc3).coord-center_curv(inc3).coord);

$\%$ for the points either not numbered first or last in the cross-section

else

midpt 1 (inc3) .coord $=($ markers (inc3-1) .coord + markers (inc3) .coord)/2;

$\mathrm{m} 1=($ markers(inc3-1) $\cdot \operatorname{coord}(3)$-markers(inc3).coord(3))/(markers(inc3-1).coord(1)-

markers(inc3).coord(1));

b1=midpt1(inc3).coord(3)+1/m1*midpt1(inc3).coord(1);

midpt2(inc3). coord=(markers(inc3+1).coord+markers(inc3).coord)/2;

$\mathrm{m} 2=($ markers $($ inc3+1) $\cdot \operatorname{coord}(3)$-markers (inc3) $\cdot \operatorname{coord}(3)) /($ markers(inc3+1).coord(1)markers(inc3).coord(1));

b2=midpt2(inc3).coord(3)+1/m2*midpt2(inc3). $\operatorname{coord}(1)$;

center_curv(inc3).coord $(1)=($ b2 $-b 1) /(-1 / m 1+1 / m 2)$;

$\mathrm{x}=(\mathrm{b} 2-\mathrm{b} 1) /(-1 / \mathrm{m} 1+1 / \mathrm{m} 2)$;

center_curv(inc3).coord (2)=markers (inc3).coord (2);

center_curv(inc3).coord (3)=b2-1/m2*x;

markers(inc3).radius_curv=norm(markers(inc3).coord-center_curv(inc3).coord); end

markers(inc3).radius_curv=markers(inc3).radius_curv/1000;

$\%$ converts from $\mathrm{mm}$ to $\mathrm{m}$ 


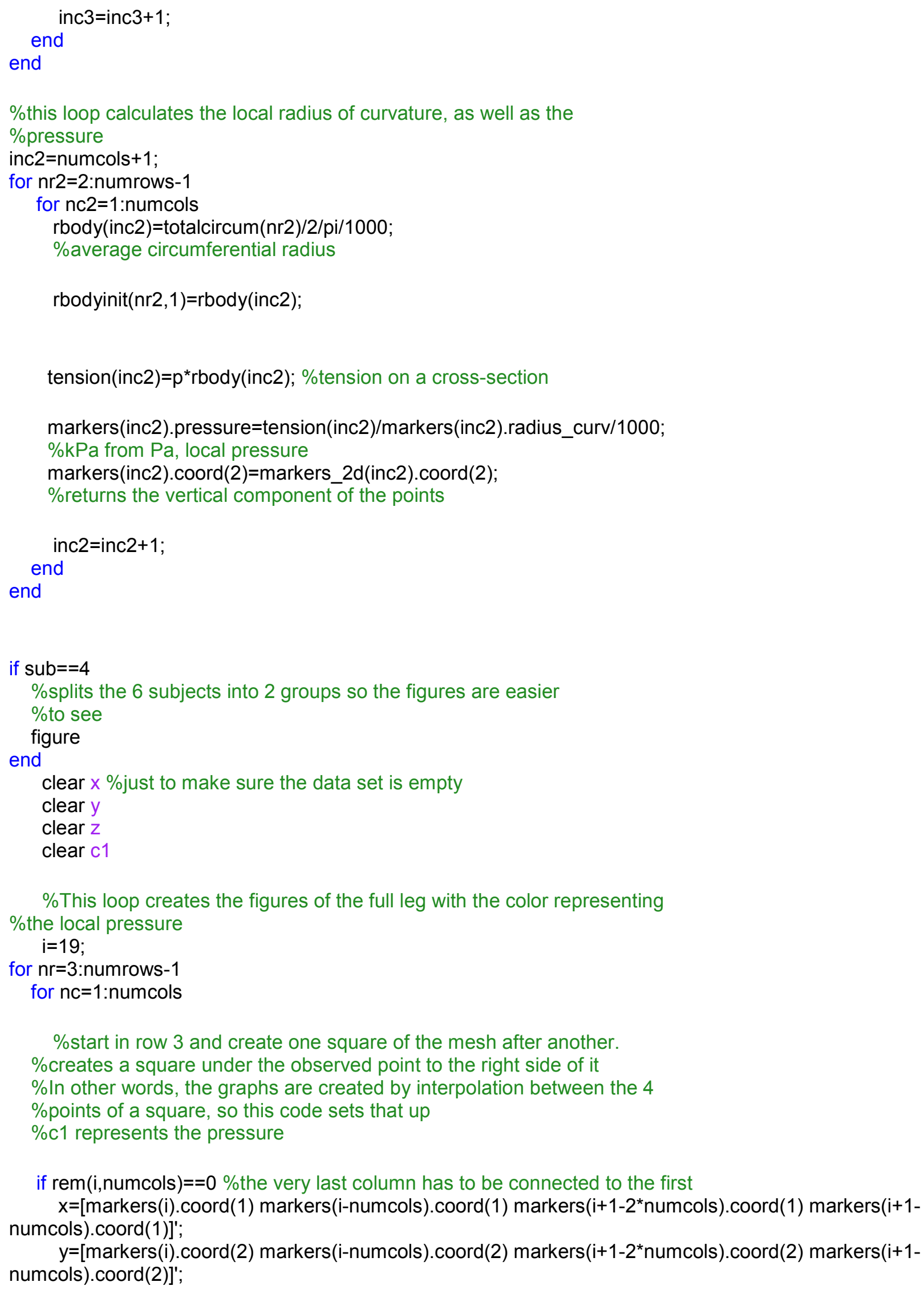


$z=[$ markers(i).coord(3) markers(i-numcols).coord(3) markers(i+1-2*numcols).coord(3) markers(i+1numcols).coord(3)]';

$\mathrm{c} 1=\left[\right.$ markers $(\mathrm{i})$. pressure markers(i-numcols). pressure markers $\left(\mathrm{i}+1-2^{*}\right.$ numcols $)$. pressure markers $(\mathrm{i}+1-$ numcols).pressure]';

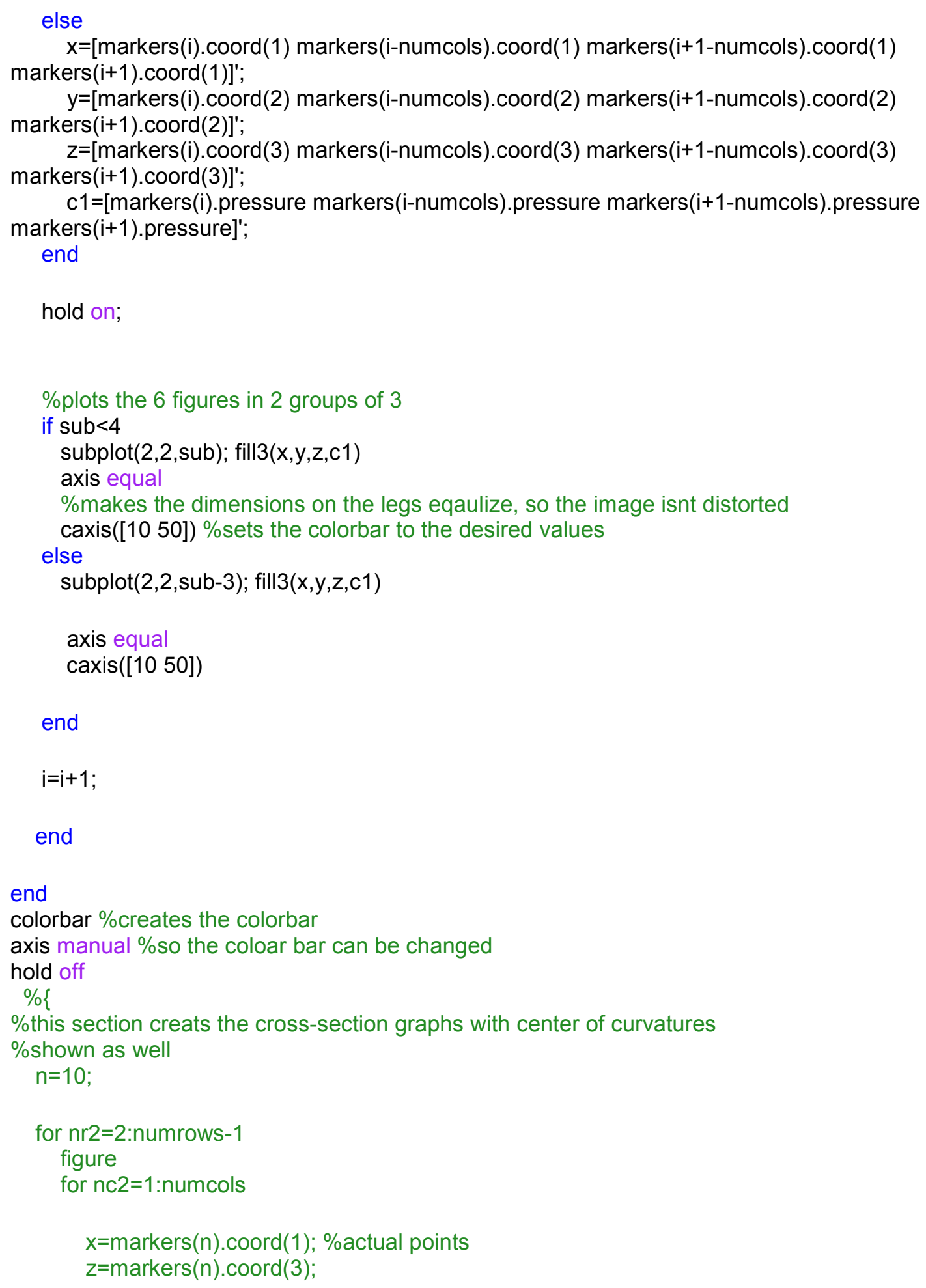




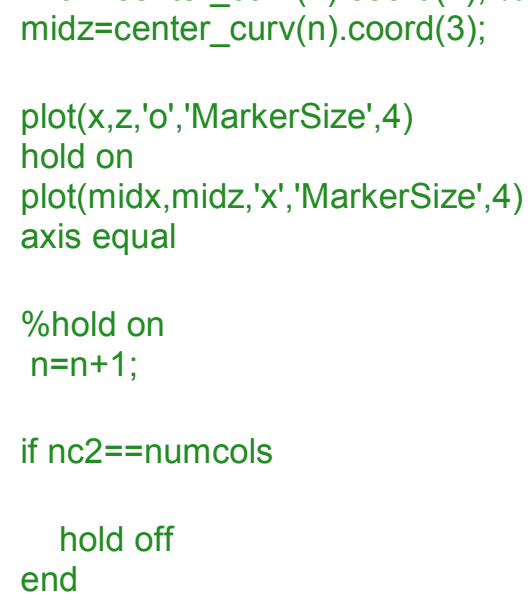




\section{Appendix F: Matlab code for analysis using Cyberware data}

\subsection{Cyberware data code}

\subsubsection{Code from Other sources}

2 sources of code not created by this author were used for this model, and were found at:

http://www.mathworks.com/matlabcentral/fileexchange/loadFile.do?objectId=5459

These files were originally created by Pascal Getreuer in 2004.

The first file is to read the .ply files which Cyberware provided, and the second is to write .ply files. For the interests of faster processing, I removed all the extraneous points from the original data set (ie everything except the right leg) and then saved as a different ply file. These are just one set of files to use, as other code is certainly available for these tasks.

\subsubsection{Custom code}

\subsubsection{1 ply Start file.m}

$\%$ plyStartfile.m

$\%$ This file starts the analysis of the Cyberware data, and calls the

\%functions Combine_xsections.m and Cross_section_analysis.m

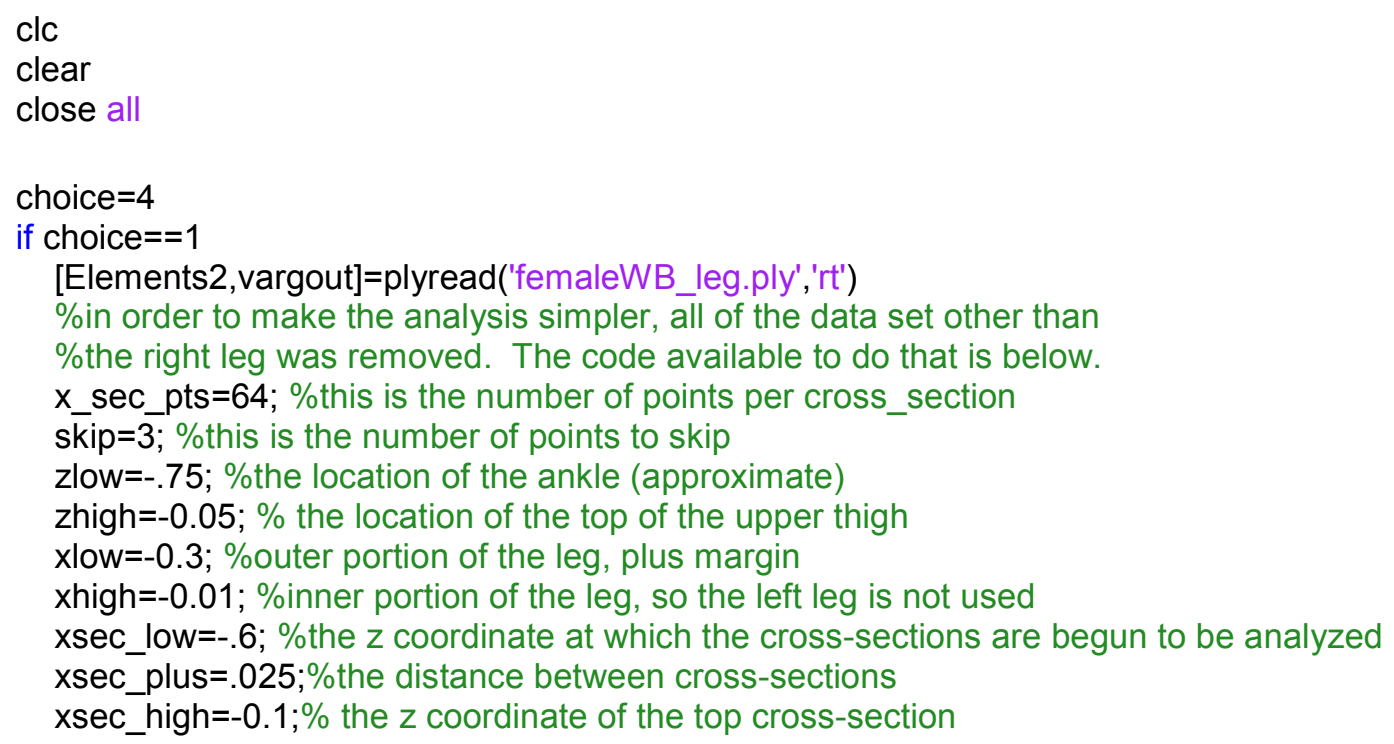




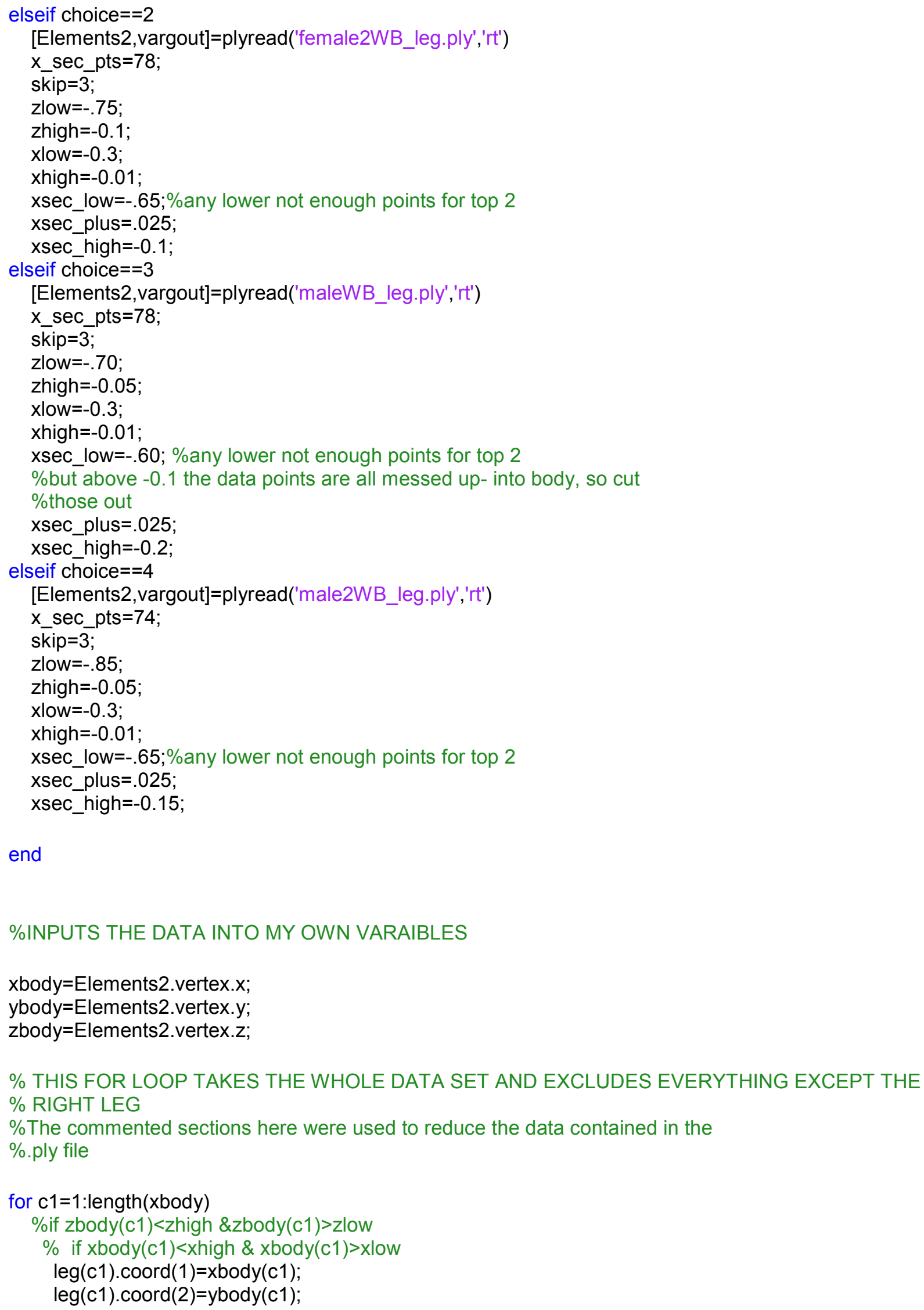




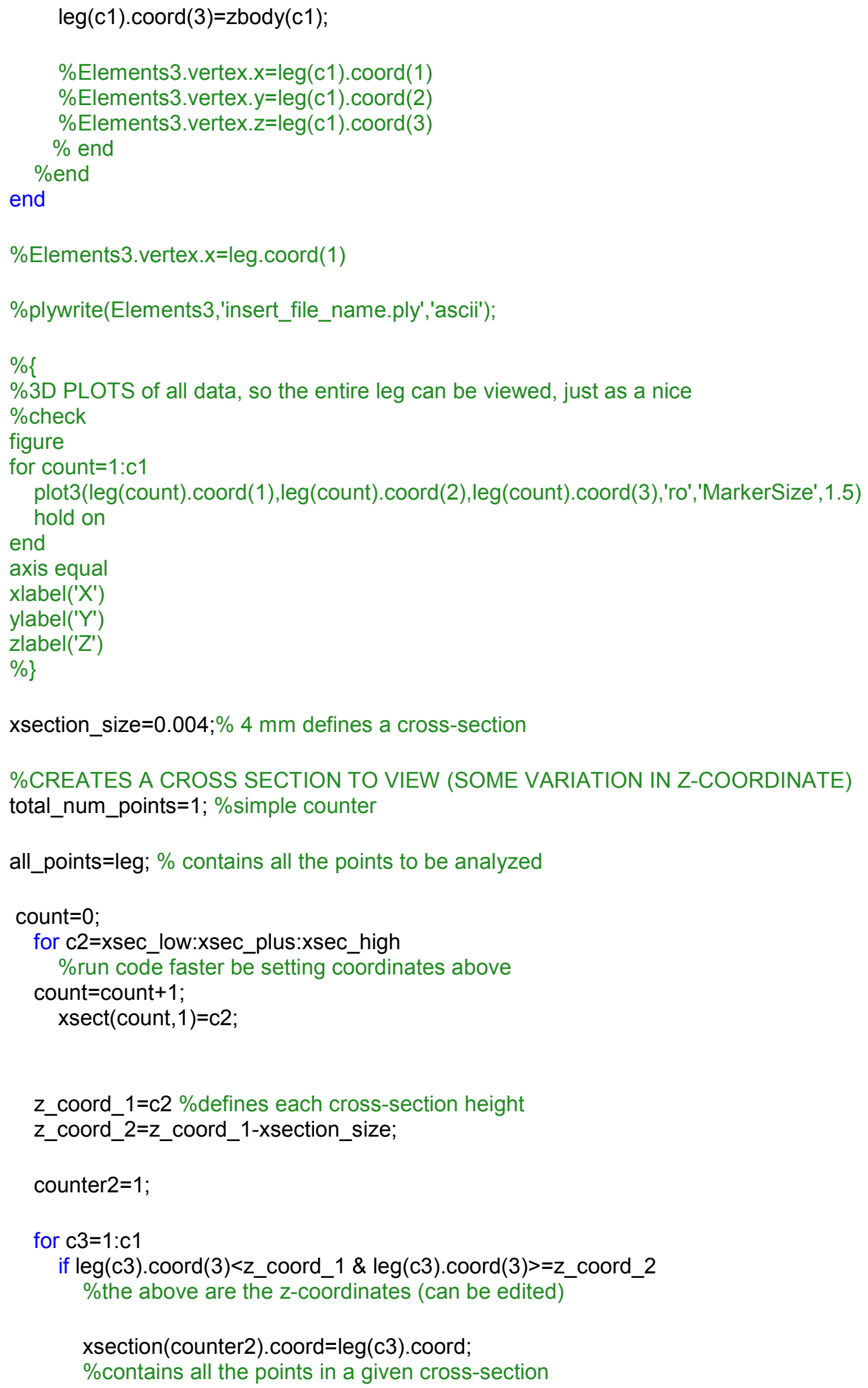




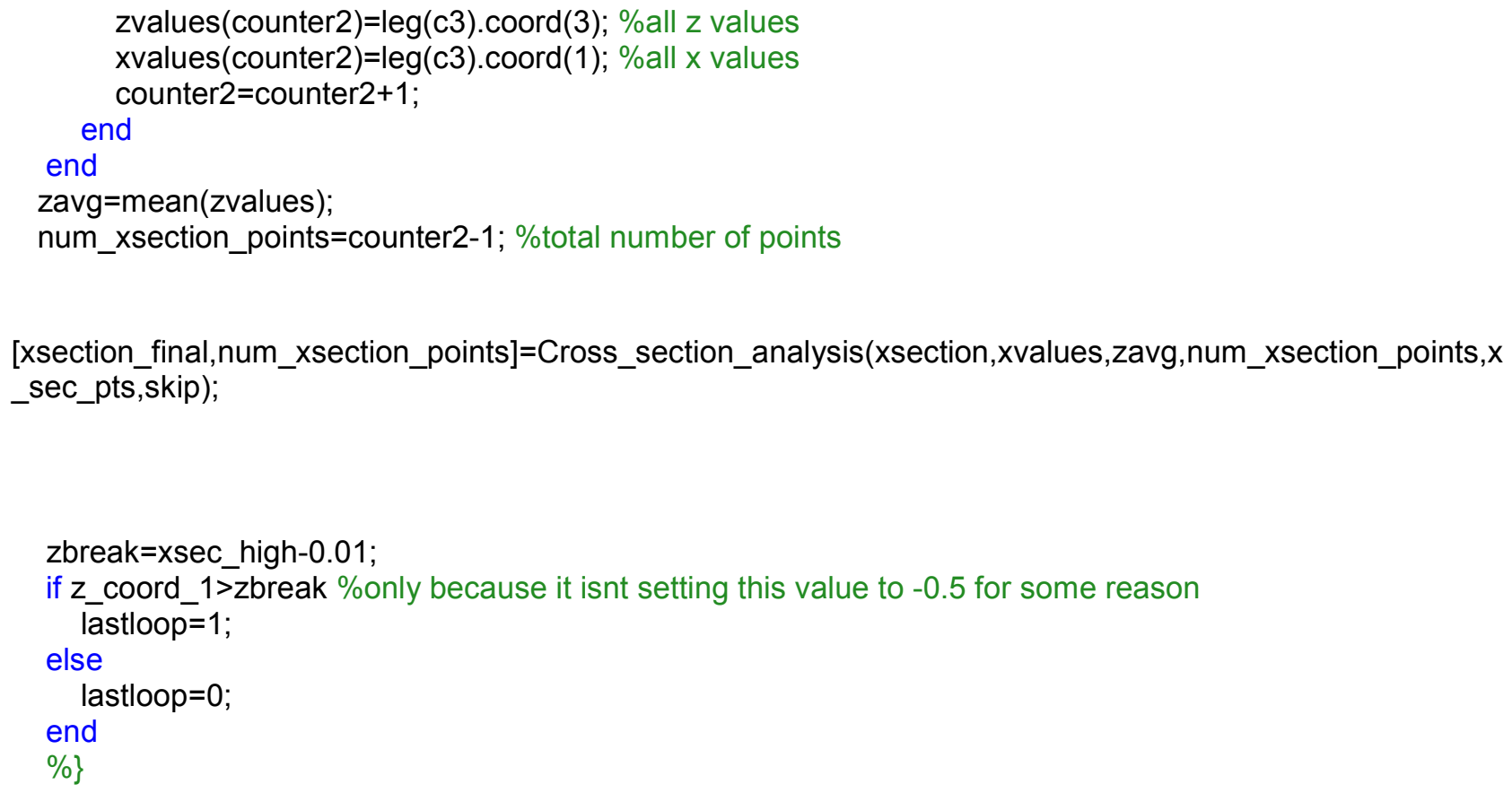

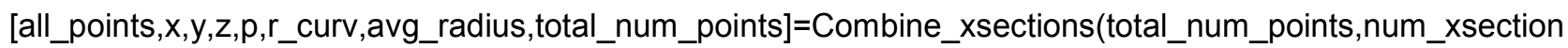
_points,xsection_final,lastloop,all_points);

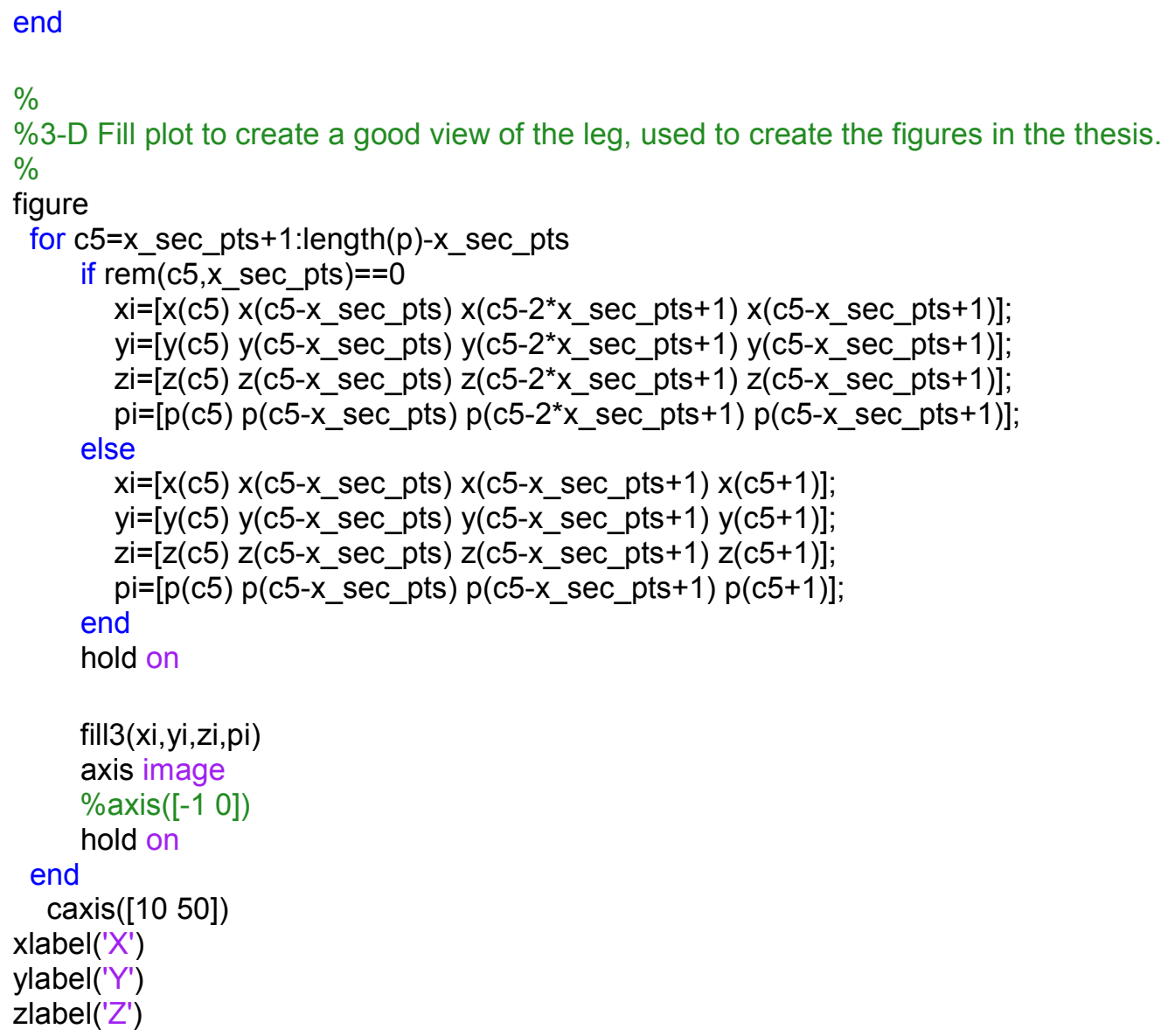




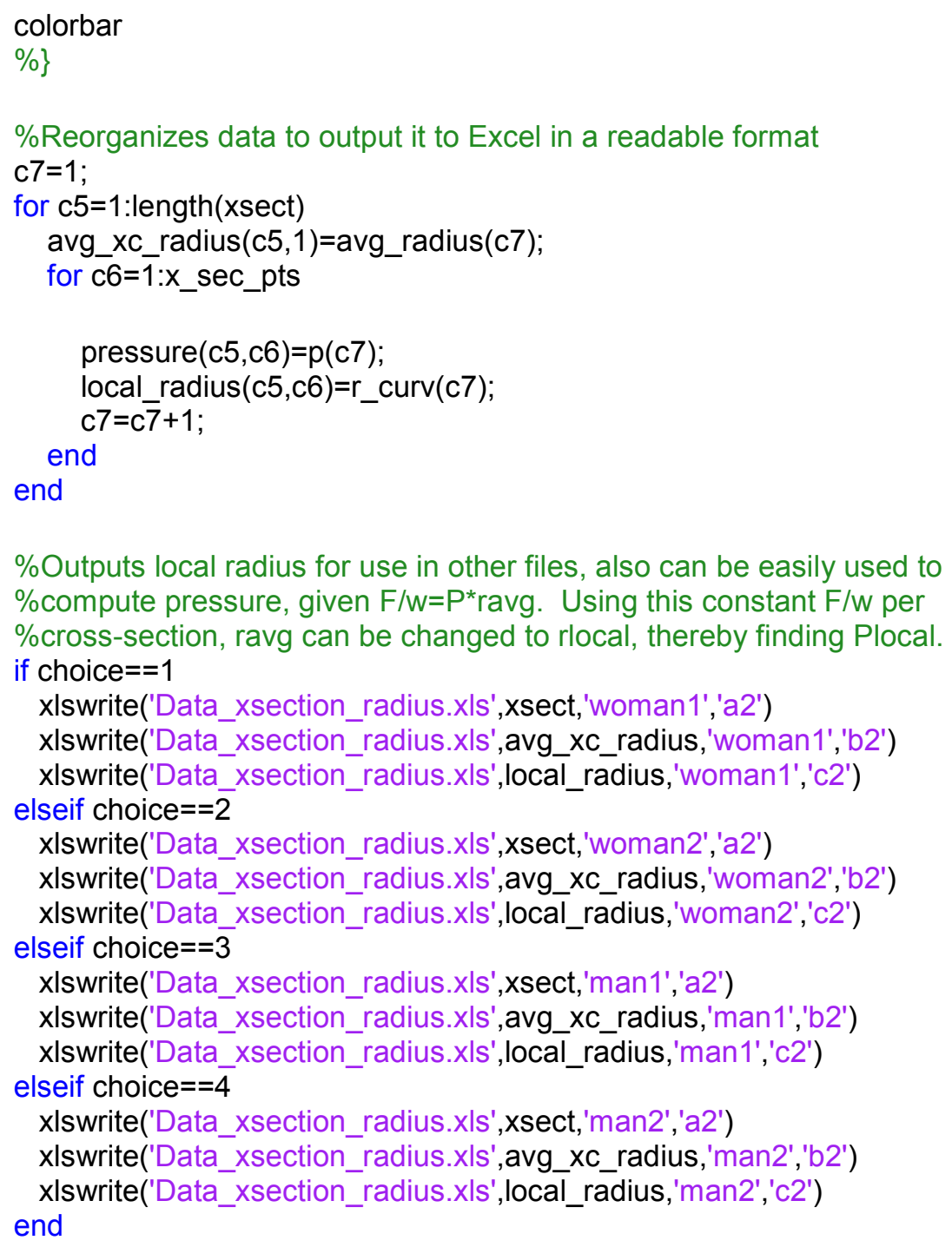

\subsubsection{Cross section analysis.m}

function

[xsection_final,add_to_final]=Cross_section_analysis(xsection,xvalues,zavg,num_xsection_points,pts_per_x section,skip)

$\%$ This function is where all the calculations occur for taking all the data

$\%$ points into a form that ccan be used to create pressure. The following

$\%$ steps are used:

$\%$ 1) Organize the files into top and bottom sections and then recombine, so

$\%$ that there is no overlap, and hte data points can be ordered

$\%$ appropriately

$\%$ 2)removes data points that are at the same radian value, but a different 
$\%$ radius so that the points represent a true cross-section. This is

$\%$ necessary since the cross-section is defined as $4 \mathrm{~mm}$ tall, which can lead

$\%$ to some slight inconsistincies in the data.

$\% 3$ ) Calculates the average radius at a given circumference

$\% 4$ ) Reduces the total number of data points at the circumference to a user

$\%$ defined value, so that the full leg graph can be made.

$\% 5$ ) Calculate the local radius of curvature

$\%\{$

figure

\%PLOT THE CROSS-SECTION, if necessary

for count2=1:num_xsection_points

plot3(xsection(count2).coord(1),xsection(count2).coord(2),xsection(count2).coord(3),'ro','MarkerSize',1.5) hold on

end

axis equal

xlabel('X')

ylabel('Y')

zlabel('Z')

$\%\}$

counter3=1;

counter4=1;

[min_x_leg,index1]=min(xvalues);

[max_x_leg,index2]=max(xvalues);

x_avg=mean(xvalues);

\%SPLITTING THE DATA INTO TOP AND BOTTOM SECTIONS FOR ORGANIZATIONAL

\%PURPOSES

for $\mathrm{c} 3=1$ :length(xvalues)

if (xsection(c3).coord(2)<=xsection(index1).coord(2)\&xsection(c3).coord(1)<x_avg)|...

(xsection(c3).coord(2)<=xsection(index2).coord(2)\&xsection(c3).coord(1)>x_avg)

$\%$ The above line puts the points where $x=\min$ and $x=\max$ in the

$\%$ bottom, so there is no overlapping of points

xsection_bottom(counter3).coord=xsection(c3).coord;

xbottom (counter3) $=x$ section $(\mathrm{c} 3) \cdot \operatorname{coord}(1)$;

counter $3=$ counter $3+1$;

else

xsection_top(counter4).coord=xsection(c3).coord;

$x$ top $($ counter 4$)=x$ section_top $($ counter 4$)$.coord $(1)$;

counter4=counter $4+1$;

end

end

order $=1$;

[xsection_bottom_ord]=organize(xbottom,xsection_bottom,order); \%1 organizes from smallest to largest x value

order=order+1;

[xsection_top_ord]=organize(xtop,xsection_top,order); \% 2 organizes largest to smallest so they all combine together well 


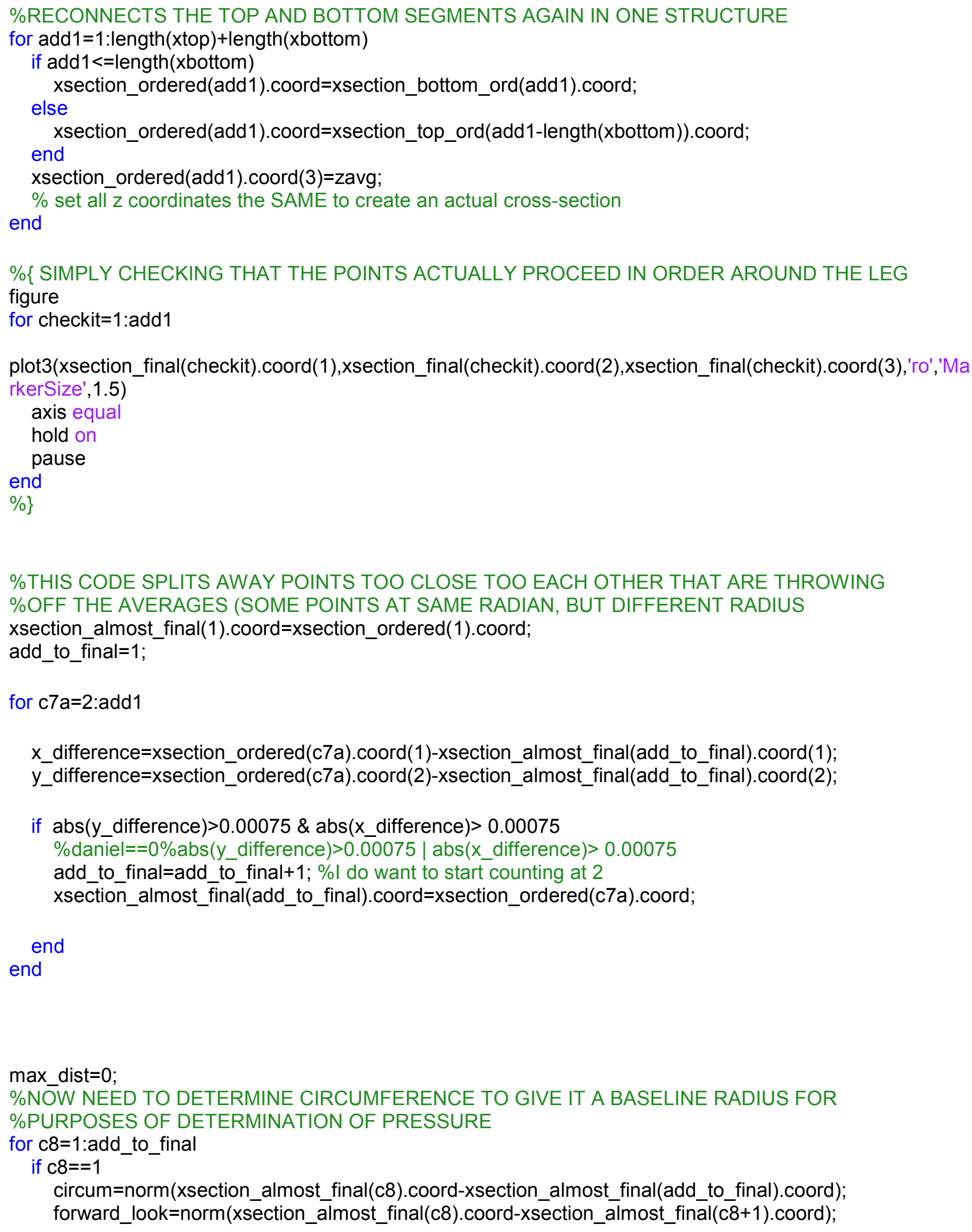




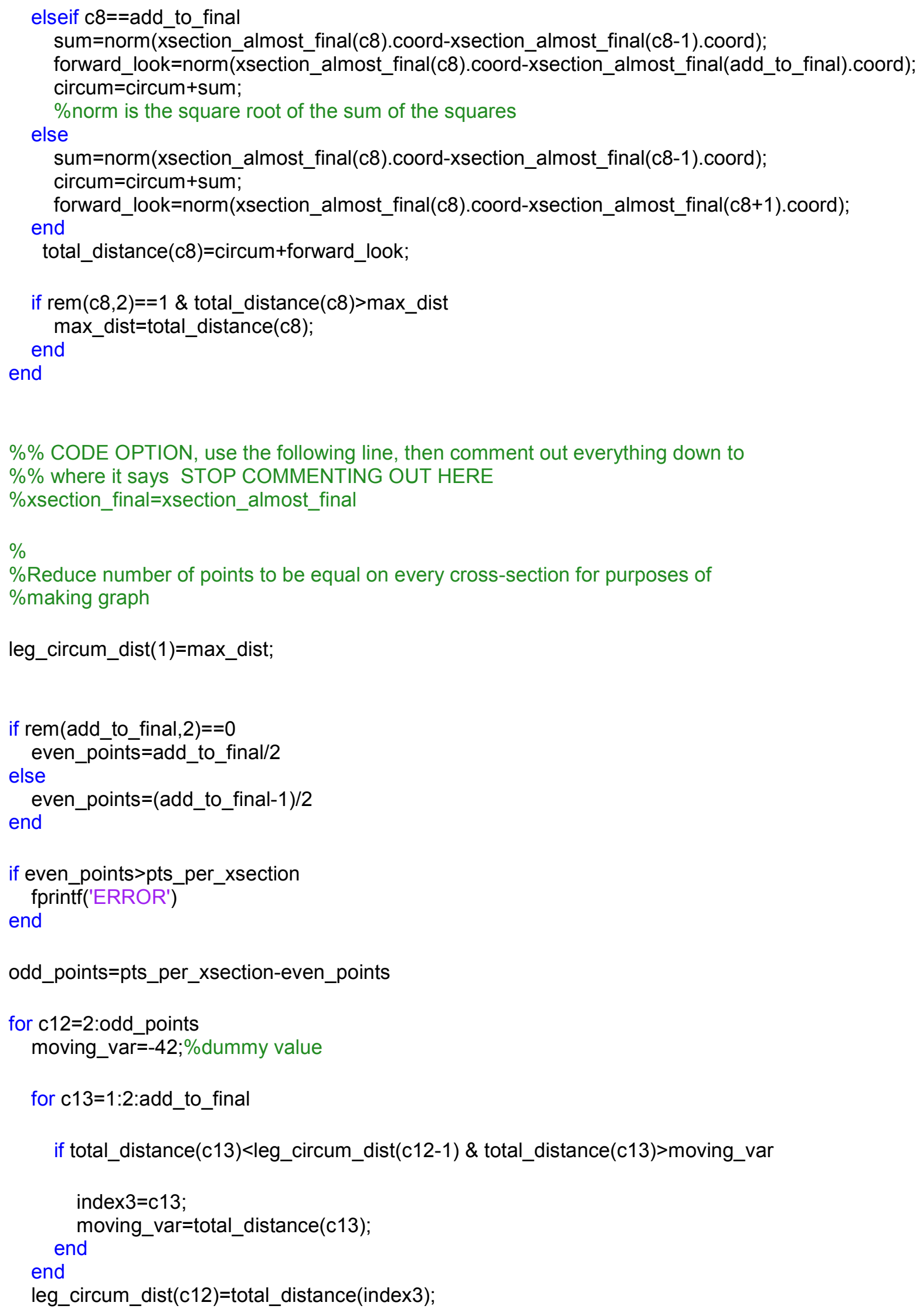




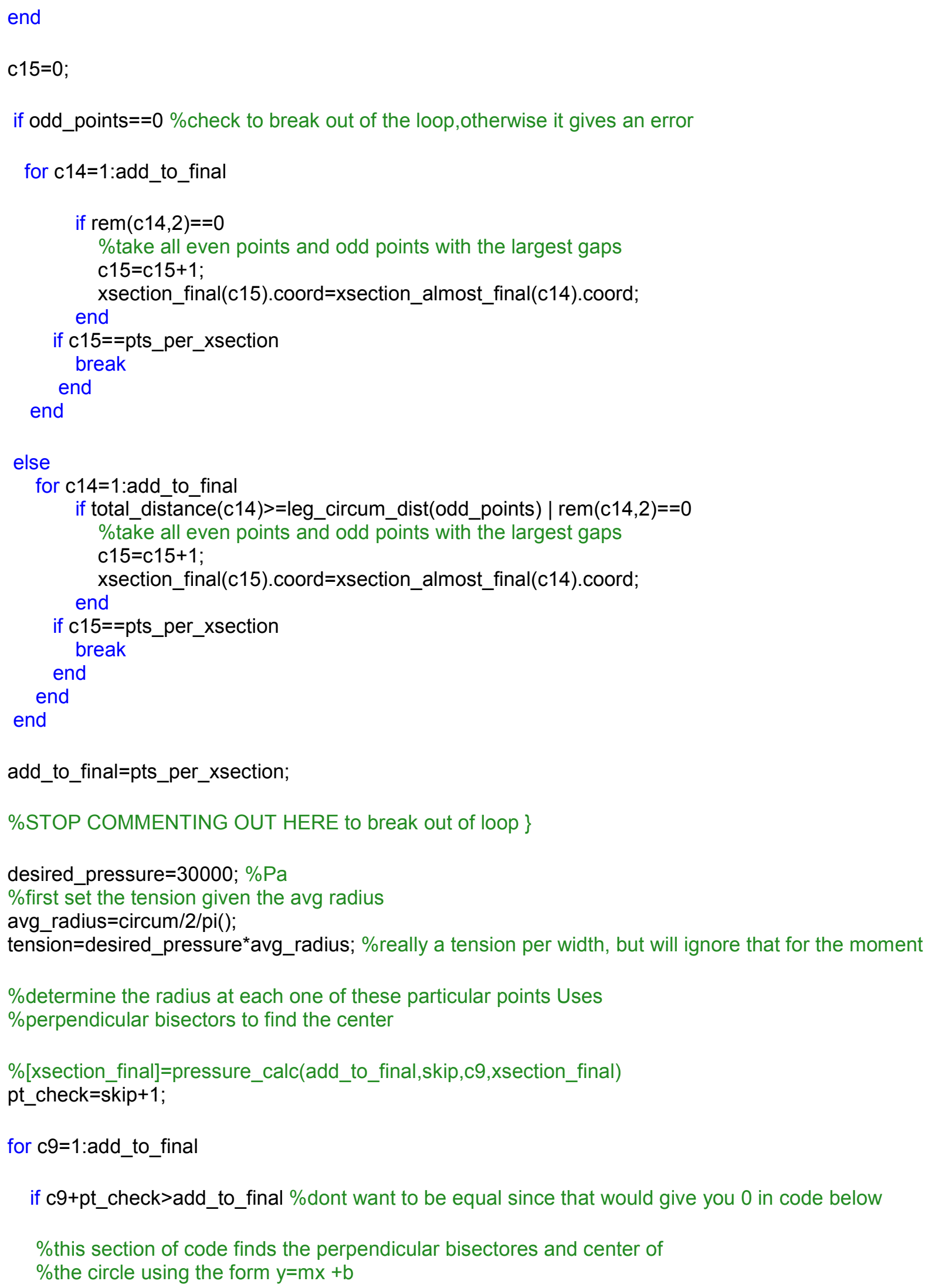


$\%$ This has the same usage as the previous code, making the necessary

$\%$ adjustments for the first and last points in the cross-section when

$\%$ finding the perpendicular bisectors based on its neighbors

gap=pt_check-(add_to_final-c9);

midpt1(c9).coord=(xsection_final(c9-pt_check).coord+xsection_final(c9).coord)/2;

$\mathrm{m} 1=\left(x s e c t i o n \_f i n a l\left(c 9-p t \_c h e c k\right) \cdot c o o r d(2)-x s e c t i o n \_f i n a l(c 9) \cdot c o o r d(2)\right) /\left(x s e c t i o n \_f i n a l(c 9-\right.$

pt_check).coord(1)-xsection_final(c9).coord(1));

b1 $=$ midpt1(c9).coord(2)+1/m1*midpt1(c9).coord(1);

midpt2(c9).coord=(xsection_final(gap).coord+xsection_final(c9).coord)/2;

$\mathrm{m} 2=\left(x s e c t i o n \_f i n a l(g a p) . c o o r d(2)-x s e c t i o n \_f i n a l(c 9) . c o o r d(2)\right) /\left(x s e c t i o n \_f i n a l(g a p) . c o o r d(1)-\right.$

xsection_final(c9).coord(1));

$\overline{\mathrm{b} 2}=\operatorname{midpt} 2(\mathrm{c} 9) \cdot \operatorname{coord}(2)+1 / \mathrm{m} 2^{*} \operatorname{midpt} 2(\mathrm{c} 9) \cdot \operatorname{coord}(1)$;

center_curv(c9).coord $(1)=(b 2-b 1) /(-1 / m 1+1 / m 2)$;

$\mathrm{x}=(\mathrm{b} 2-\mathrm{b} 1) /(-1 / \mathrm{m} 1+1 / \mathrm{m} 2)$;

center_curv(c9).coord(3)=xsection_final (c9).coord(3);

center_curv(c9).coord $(2)=\mathrm{b} 2-1 / \mathrm{m} 2^{\star} \mathrm{x}$;

elseif c9-pt_check $<=0$

gap=add_to_final-(pt_check-c9);\%need to get to 0 here

midpt1(c9).coord=(xsection_final(gap).coord+xsection_final(c9).coord)/2;

m1=(xsection_final(gap).coord(2)-xsection_final(c9).coord(2))/(xsection_final(gap).coord(1)xsection_final(c9).coord(1));

$\overline{b 1}=\operatorname{midpt} 1(\mathrm{c} 9) \cdot \operatorname{coord}(2)+1 / \mathrm{m} 1{ }^{*} \operatorname{midpt} 1(\mathrm{c} 9) \cdot \operatorname{coord}(1)$;

midpt2(c9).coord=(xsection_final(c9+pt_check).coord+xsection_final(c9).coord)/2;

$\mathrm{m} 2=$ (xsection_final(c9+pt_check).coord(2)-

xsection_final(c9).coord(2))/(xsection_final(c9+pt_check).coord(1)-xsection_final(c9).coord(1)); b2=midpt2(c9).coord(2)+1/m2*midpt2(c9). coord(1);

center_curv(c9).coord $(1)=(b 2-b 1) /(-1 / m 1+1 / m 2)$;

$\mathrm{x}=(\mathrm{b} 2-\overline{\mathrm{b}} 1) /(-1 / \mathrm{m} 1+1 / \mathrm{m} 2)$;

center_curv(c9).coord (3)=xsection_final(c9).coord(3);

center_curv(c9).coord $(2)=b 2-1 / \mathrm{m} 2^{\bar{*}} \mathrm{x}$;

else

midpt1(c9).coord=(xsection_final(c9-pt_check).coord+xsection_final(c9).coord)/2;

$\mathrm{m} 1=\left(x s e c t i o n \_f i n a l\left(c 9-p t \_c h e c k\right) . c o o r d(2)-x s e c t i o n \_f i n a l(c 9) . c o o r d(2)\right) /\left(x s e c t i o n \_f i n a l(c 9-\right.$

pt_check).coord(1)-xsection_final(c9).coord(1));

b1 $=\operatorname{midpt} 1(\mathrm{c} 9) \cdot \operatorname{coord}(2)+1 / \mathrm{m} 1{ }^{*} \operatorname{midpt} 1(\mathrm{c} 9) \cdot \operatorname{coord}(1)$;

midpt2(c9).coord=(xsection_final(c9+pt_check).coord+xsection_final(c9).coord)/2;

$\mathrm{m} 2=($ xsection_final $(c 9+$ pt_check).coord $(2)-$

xsection_final(c9).coord(2))/(xsection_final(c9+pt_check).coord(1)-xsection_final(c9).coord(1)); b2 =midpt2(c9).coord (2)+1/m2* midpt2(c9).coord(1);

center_curv(c9).coord $(1)=(b 2-b 1) /(-1 / m 1+1 / m 2)$;

$\mathrm{x}=(\mathrm{b} 2-\overline{\mathrm{b}} 1) /(-1 / \mathrm{m} 1+1 / \mathrm{m} 2)$;

center_curv(c9).coord(3)=xsection_final(c9).coord(3);

center_curv (c9).coord $(2)=b 2-1 / m 2^{\bar{*}} \mathrm{x}$; 
end

xsection_final(c9).radius_curv=norm(xsection_final(c9).coord-center_curv(c9).coord);

xsection_final(c9).pt_pressure= tension/xsection_final(c9).radius_curv;

xsection_final(c9).avg_radius=avg_radius; \%final average radiues

rdiff(c9)=xsection_final(c9).radius_curv-avg_radius;

end

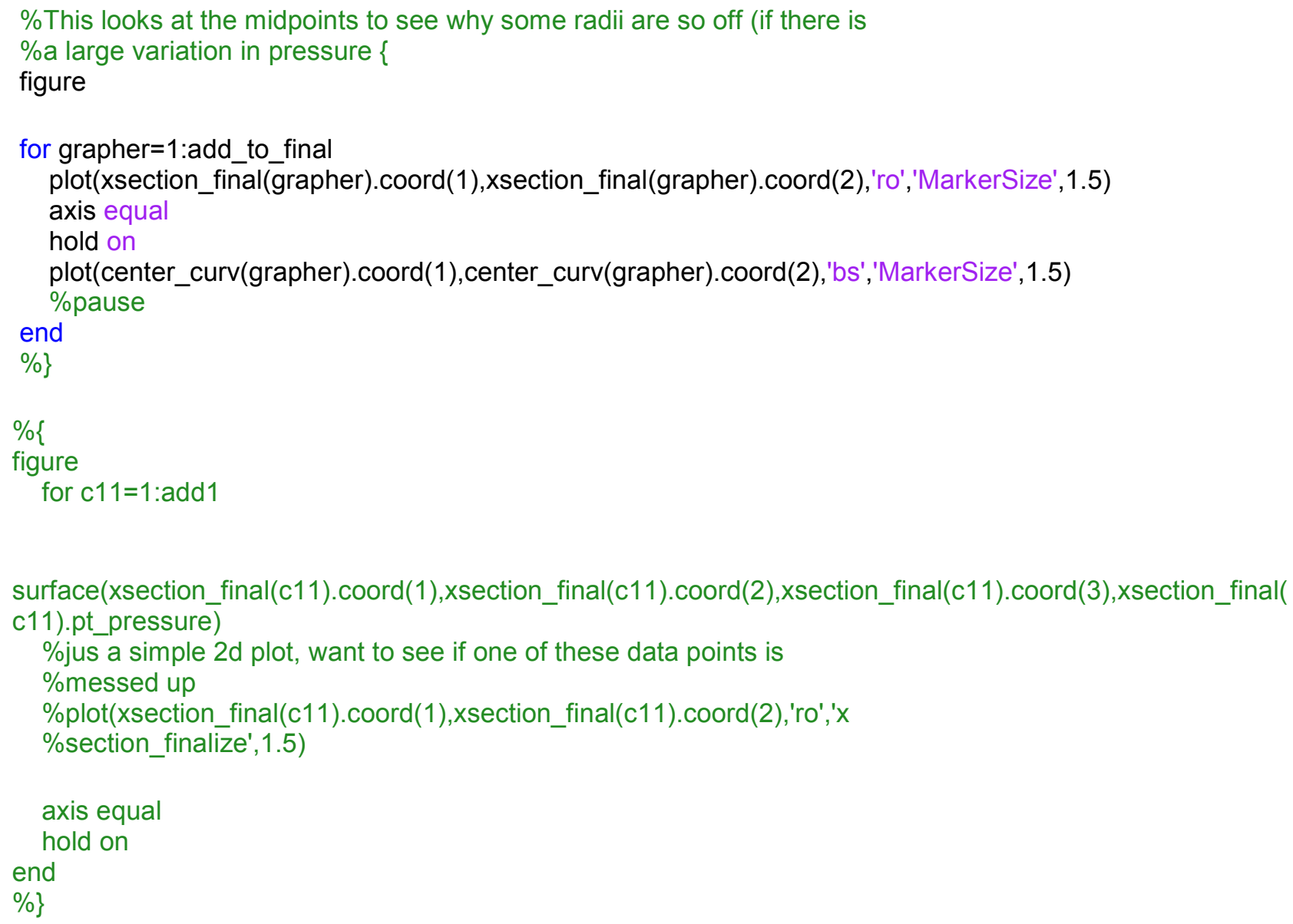

\subsubsection{Combine cross sections.m}

function

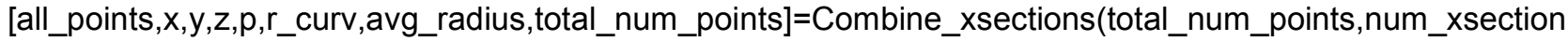
_points,xsection_final,lastloop,all_points)

$\%$ This file combines all the cross-sections together into strcuture, since

$\%$ the the function "Cross_section_analysis" only takes one cross-section at

$\%$ a time

for c1=1:num_xsection_points \%this loop adds the points all together 


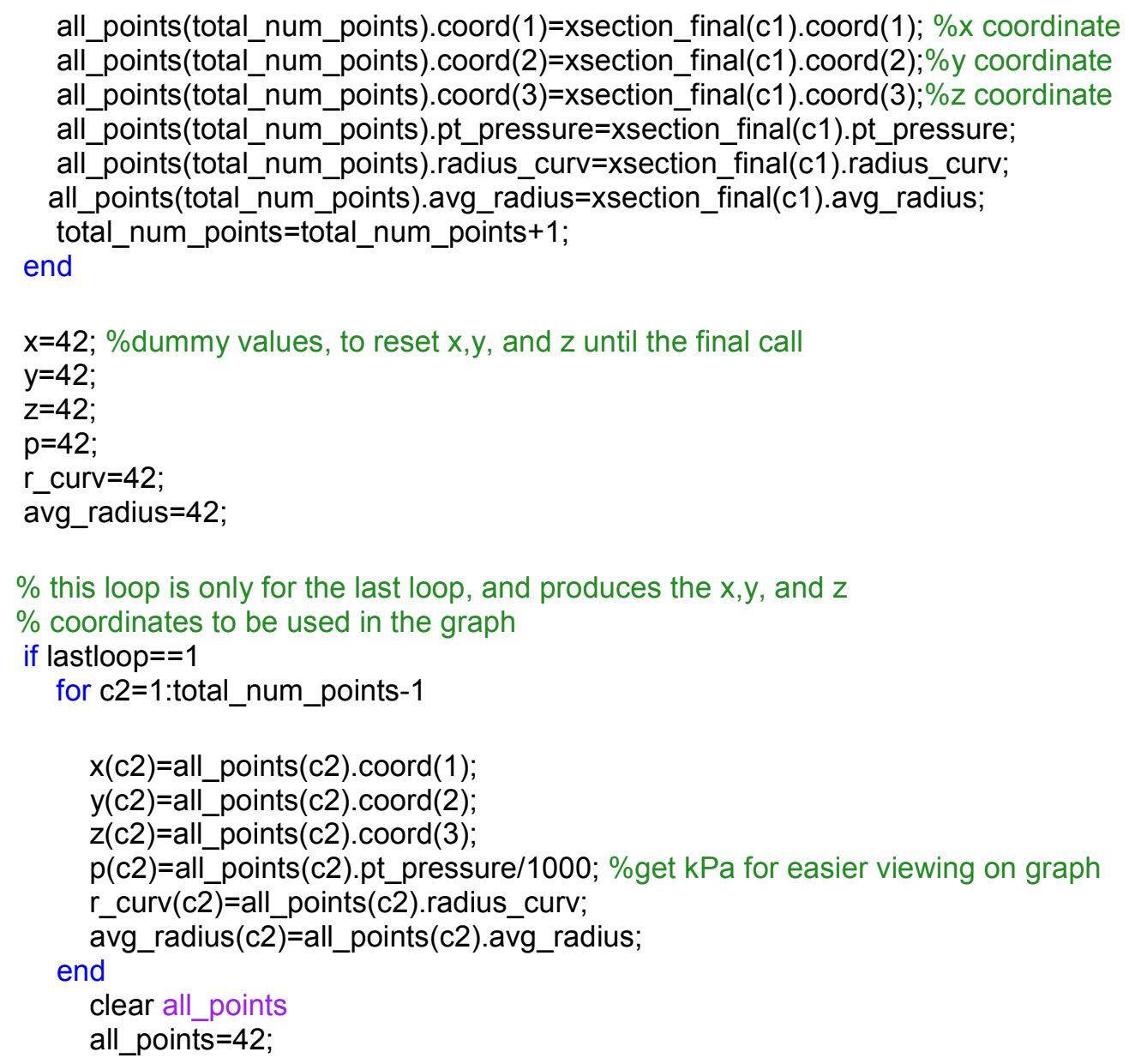

end 


\section{Appendix G: TekScan procedure}

This appendix outlines the treatment that each sensor must undergo when ordered, as well as the procedure to be followed before each test. This is the same procedure followed by Sim in his thesis, albeit more detailed, with the exception of choosing $150 \mathrm{~mm} \mathrm{Hg}$ instead of $100 \mathrm{~mm} \mathrm{Hg}$ as the lower calibration point.

\subsection{Initial procedures}

- $\quad$ Buy Model 9801, choose the 35 psi $(241.3 \mathrm{kPa})$ version

O available at: http://www.tekscan.com/medical/catalog.html

- Peel off protective backing

- Slice between the columns of the sensors from the small hole near the portion of the sensor "neck" all the way to the opposite end

- Prick each square sensel with a pin to allow any trapped air to escape, but be sure to avoid any of the electronics

- Cover the sensor with a sheet of plastic (see figure below) to protect the sensor from damage, also reduces shear strain

- Tape the sensor to the plastic at both the beginning of the neck area and at the bottom to ensure best readings

- Precondition sensor by loading it for at least $30 \mathrm{~s}$ at $>400 \mathrm{~mm} \mathrm{Hg}$ three or more times by following the procedure below

○ note: gauge only reads up to $300 \mathrm{~mm} \mathrm{Hg}$, so a guess can be made

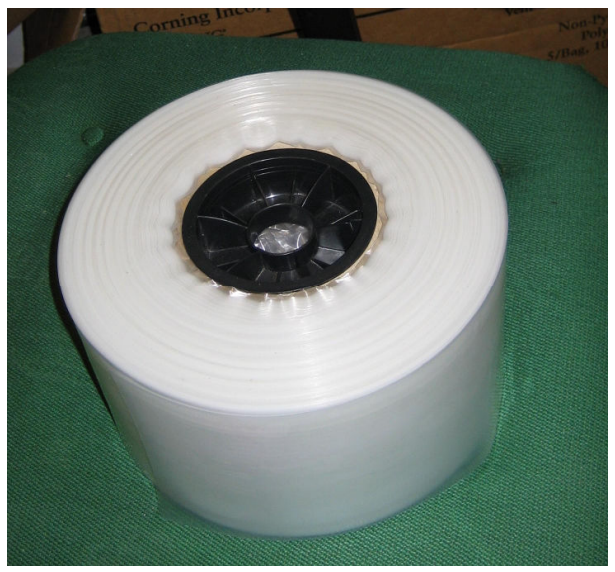

Figure 92: Plastic roll to cover TekScan system

\subsection{Tasks prior to testing}

Equilibration and calibration, as described below, need to be redone if they have not been accomplished within 2-3 hours before the test. 
- Sign on to dedicated computer by pressure chamber: password is "mcpwebb"

- Click on IScan

- Place sensor and pressure bladder in the calibration cylinder (or flat plate)

○ the sensor neck and the bolt in the bladder should be on opposite sides of the calibration device to allow for ease of movement of the sensor, and better pressure measurements

- Place sensor neck into handle, make sure to push in all the way

- 3 sensels will not read, this is an issue with handle

- Go to Tools and click on Adjust Sensitivity

- click on Mid-3 (best balance of accuracy and range- up to $500 \mathrm{~mm} \mathrm{Hg}$ )

- Go to Tools and click on Equilibration

○ this setting makes sure all sensels have same sensitivity, so it adjusts reading of each sensel

0 in order, equilibrate at the following values:

- $50,100,150,200,250$, and $300 \mathrm{~mm} \mathrm{Hg}$

- these 6 points (out of a maximum of 10) are considered a reasonable method for equilibrating

- allow $30 \mathrm{~s}$ for equilibration (more is better, but is not necessary)

- if the sensel is black, it is not reading any pressure

- the exact values that are used for equilibration are not important (ie $47 \mathrm{~mm}$ $\mathrm{Hg}$ is as useful as $50 \mathrm{~mm} \mathrm{Hg}$ ), but a good range is needed

- the bladder should be checked for leaks, as if it is leaking too quickly, then it will affect the results due to the hysteresis

- save equilibration file as ASCII file in case computer crashes

- Go to Tools and click on calibration

- Calibration sets the pressure that the sensel is measuring, so it is critical that the pressure produced is stated accurately, or else the TekScan sensor will not read the pressure properly

- click add file and choose 2 calibration points

- 150 , then $300 \mathrm{~mm} \mathrm{Hg}$

- since we are shooting for $225 \mathrm{~mm} \mathrm{Hg}$, we want 2 points roughly equidistant from the expected pressure, at least $75 \mathrm{~mm} \mathrm{Hg}$ away from that point, as stated in the TekScan manual

- set calibration delay to $120 \mathrm{~s}$ to allow for sensor stabilization

- Notes: raw sum can't be greater than 25,000

- To calculate the force to enter for calibration, follow these steps

- Divide the pressure (either 150 or $300 \mathrm{~mm} \mathrm{Hg}$ ) by the number of total working sensels (maximum of 96 if all sensels are working)

○ multiply by the sensel area: 0.25 in $^{2}$

○ multiply by $0.0193367747 \mathrm{psi} / \mathrm{mm} \mathrm{Hg}$ to get $\mathrm{lb}$

○ for $150 \mathrm{~mm} \mathrm{Hg}$ : load is $69.6 \mathrm{lb}$, for $300 \mathrm{~mm} \mathrm{Hg}$ is $139.22 \mathrm{lb}$

- save calibration file

- You can take the sensor out during the process then place back in after calibration, can reload file if computer crashes 


\subsection{Taking data during a test}

- Go to file and take a snapshot

- save ASCII (use defaults)

- Open Excel and open the file you just saved

- use delimited (not fixed width)

- hit next

- click on commas (under delimited options)

- click next

- click finish 


\section{Appendix H: Bladder creation procedure}

The following steps detail how the bladders used for the TekScan testing were created.

\subsection{Materials}

- 2 pieces of plastic (ST-1880, 0.010" by 36" Surface G-2, Stevens Urethane)

- Heat sealer

- 1 Bulkhead fitting (Part No. 5454K85, McMaster-Carr, New Brunswick NJ)

- Double-Bubble Urethane adhesive (Part No. 7493A21, McMaster-Carr)

- 2 o-rings with ID: 0.487" and OD: 0.693", can be either:

- (Part No. 9464K25, McMaster-Carr)

○ (Part No. 94115K112, McMaster-Carr)

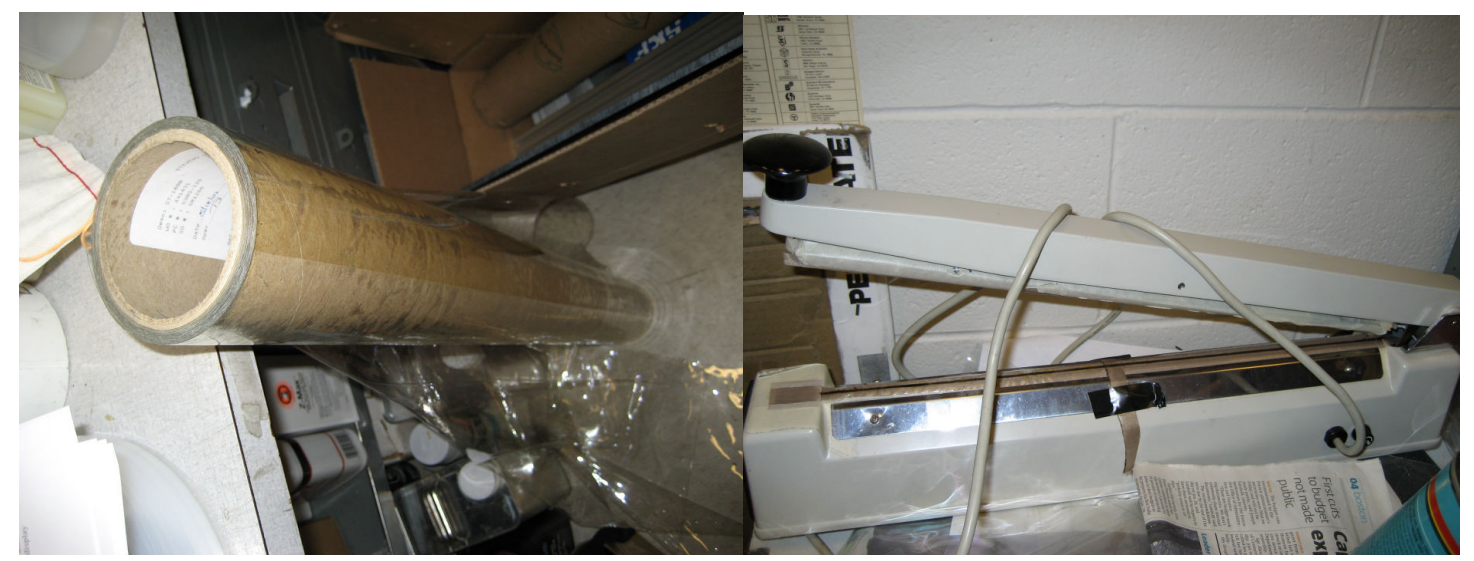

Figure 93: (left) Plastic used for bladders

Figure 94: (right) Heat sealer

\subsection{Procedure}

- cut 2 pieces of plastic, approximately 15 " by 7 "

0 can trace over current bladders as a shortcut

- cut a small triangular hole on the major axis near the edge of the material

- place in the bolt and nut

- clean the bolt, nut, and surface of the plastic with rubbing alcohol for a better seal

- put in the 2 o-rings, as shown in Figure 95

- use Double-Bubble urethane to seal over the o-ring and bulkhead on the outside of bladder material

- use Double-Bubble urethane to seal over the o-ring and nut on the inside of bladder material

- let it dry 


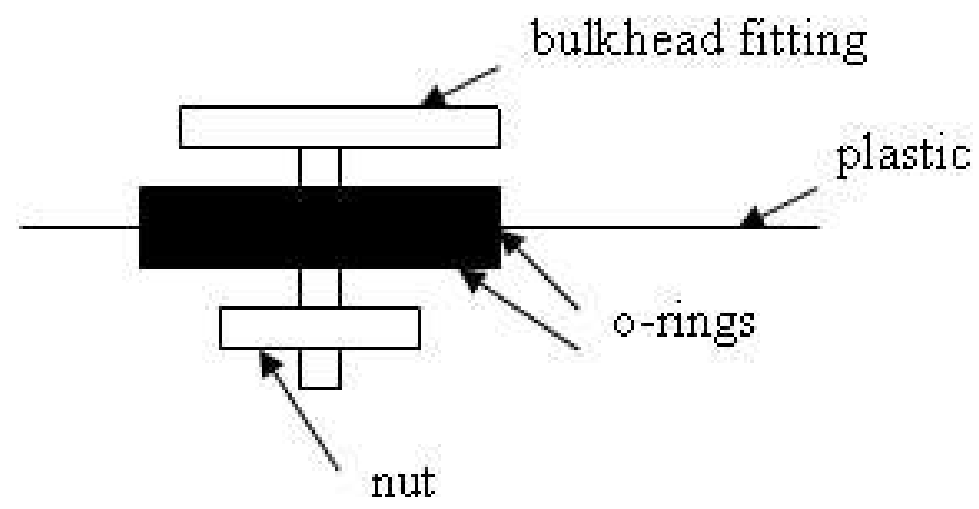

Figure 95: O-rings with bulkhead fitting on bladder

- $\quad$ seal the 2 pieces of plastic together with a heat sealer

$\circ$ the highest setting ( 8 on the pictured model) works well, but this may vary with other heat sealers

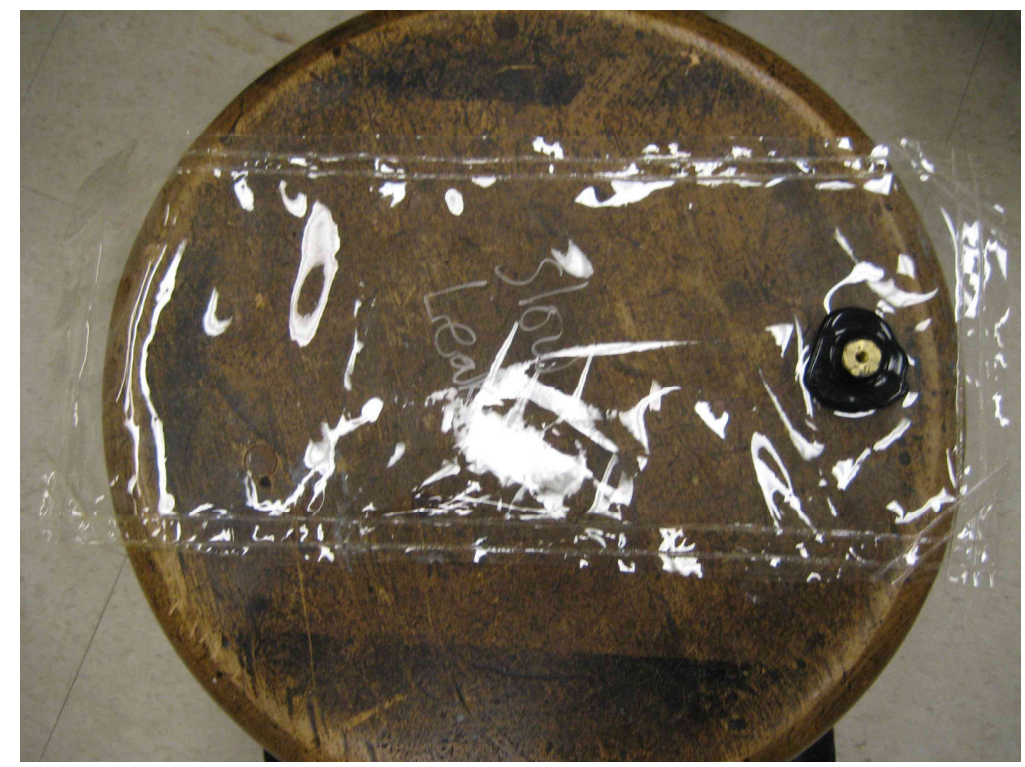

Figure 96: Finished bladder 


\section{Appendix I: Calibration of elastic bands}

The calibration for the elastic bands was a multiple step process, as outlined below. The first section will outline the procedure, while the second section will display the Matlab code used to create the bands.

\subsection{Procedure overview}

\subsubsection{Phase 1: material stretching}

The first step was to take each of the elastic bands and stretch them, using about $12-14 \mathrm{lbf}^{26}$ as some minor permanent deformation occurred after these tests. After this initial stretching occurred, the bands were stretched at a series of forces: 2, 4, 6, 8, and $10 \mathrm{lbf}$ with the strain gauge seen below and both the width and length changes were measured. These measurements were conducted at 2 places on the band (opposite ends) to ensure the material properties were constant. After a number of tests, it became clear that the material properties were all approximately the same (the material would creep under a force, so there is some error inherent in the measuring process), so the results were averaged for all bands. This made the calculations in the following section much easier, since rather than doing separate calculations for each band, only one set was conducted.

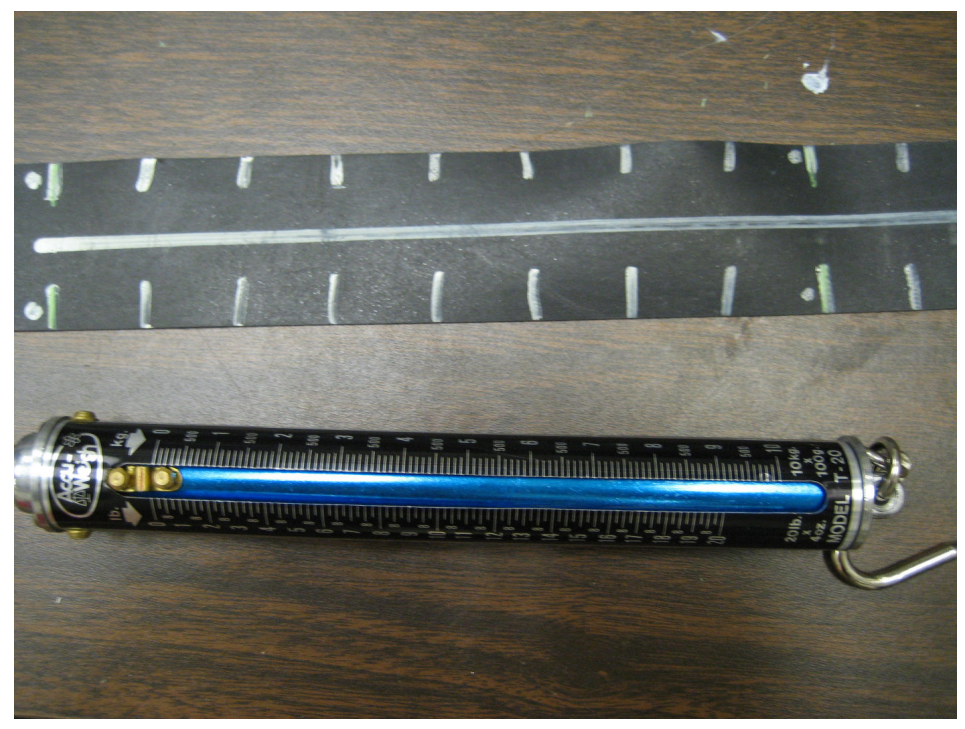

Figure 97: Example of marked band (top) and strain gauge (bottom)

\footnotetext{
${ }^{26}$ Stretching the bands too hard will cause them to deform plastically, which makes them useless for material testing, so no force higher than this should be used.
} 
The Young's Modulus of the current material ranges between 1.8 to $2.8 \mathrm{MPa}$, depending on the strain applied. The stress-strain curve for the neoprene rubber can be seen in the following figure.

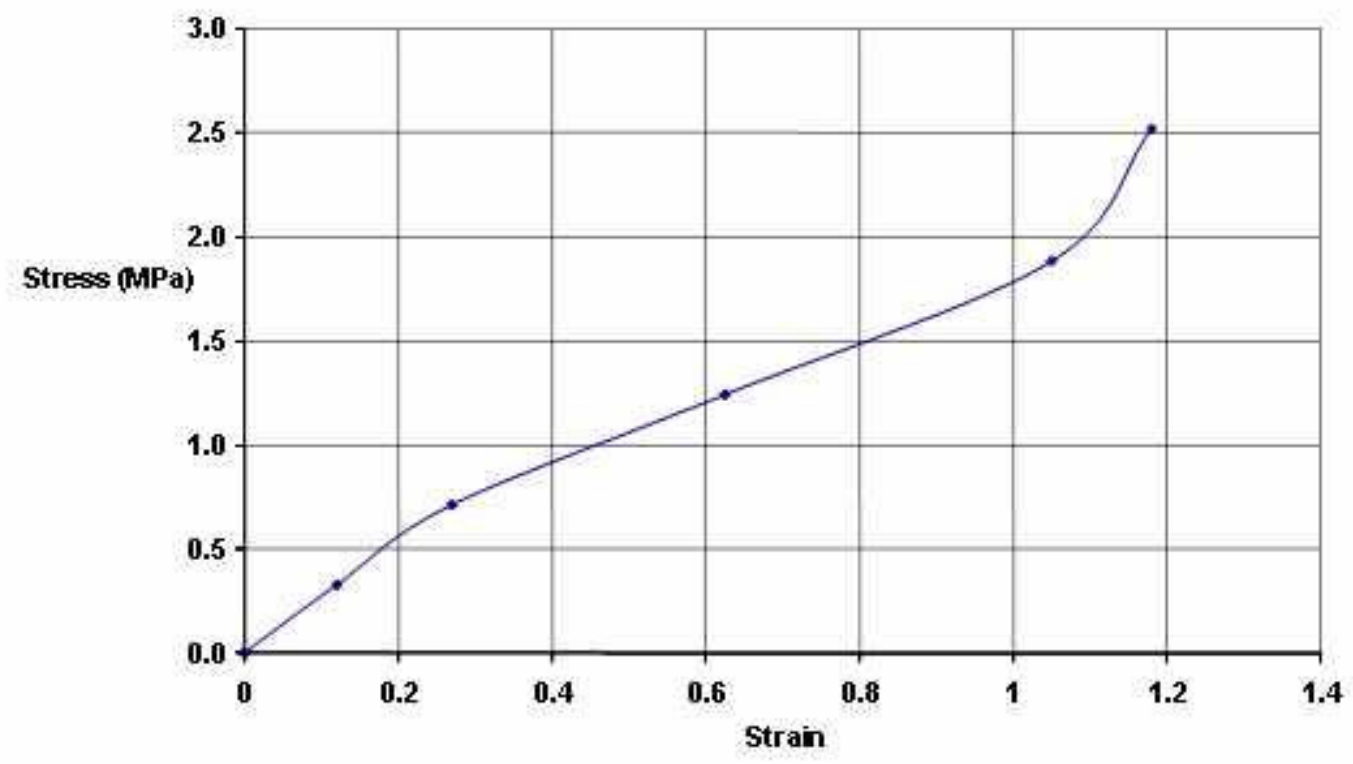

Figure 98: Stress-strain curve for neoprene rubber used for current experiments

These values are somewhat different from those in Table 6 in Chapter 2. However, those calculations are assuming the much smaller closure distances, and hence significantly smaller strains. The neoprene bands are routinely stretched $15-20 \mathrm{~cm}$ per cross-section, far more than the requirement for the operational material (a maximum of $5 \mathrm{~cm}$ ).

\subsubsection{Phase 2: initial calculations}

The force per unit width was compared against the strain in the direction of the length for the various forces, and then an excel spreadsheet was set up to establish a trendline for the data. That trendline and the data points are shown in the figure below. 


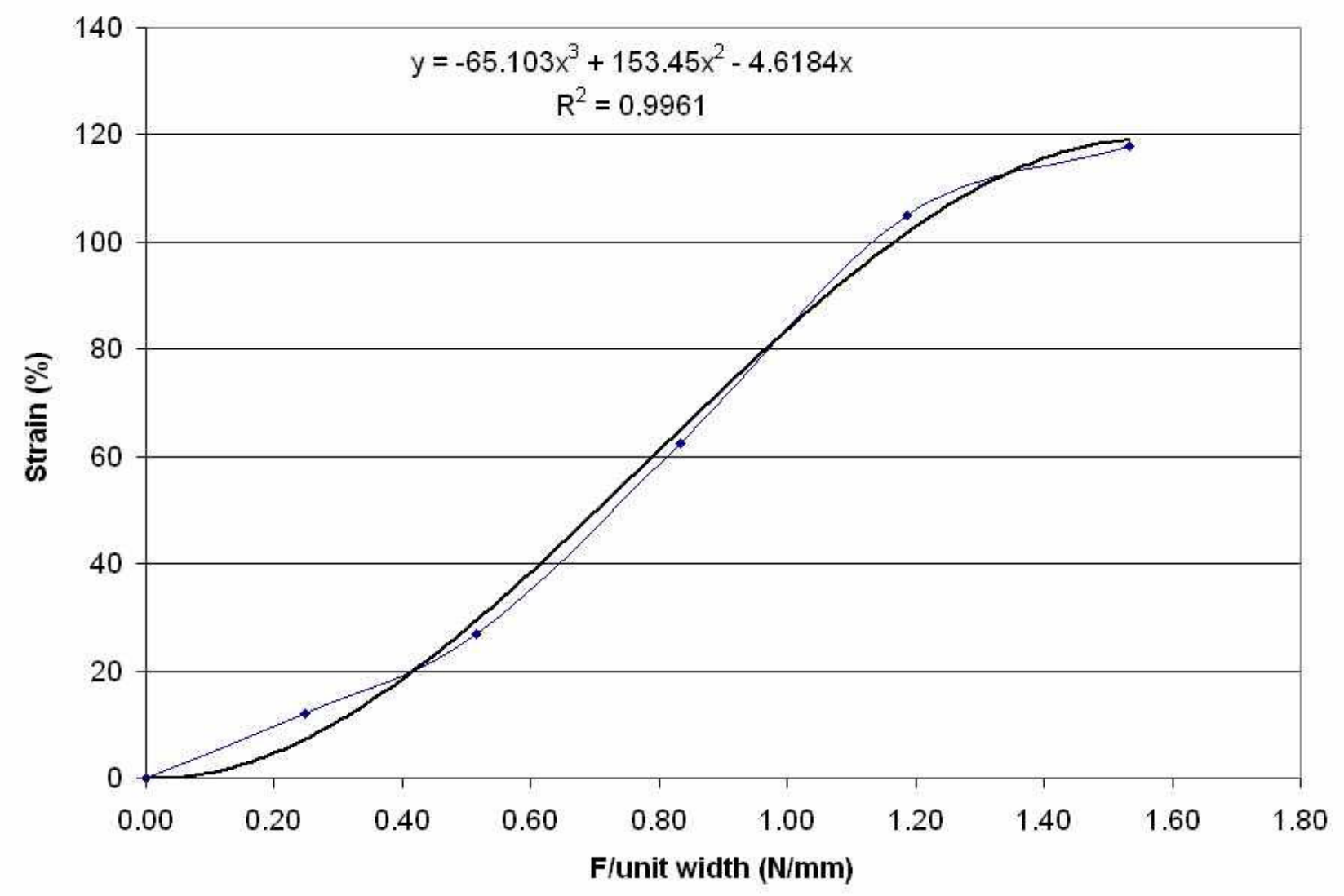

Figure 99: Strain vs. force per width

\subsubsection{Phase 3: human leg measurements}

Measurements were taken of the circumferences of each of the subject's right leg in $2 \mathrm{~cm}$ vertical increments. The center of the ankle was considered to be $0 \mathrm{~cm}$, in order to normalize it to all the subjects. A better method would probably be to start measuring from where the initial wrap begins (typically around $4 \mathrm{~cm}$ above the ankle), but this was not realized until later.

\subsubsection{Phase 4: Matlab code overview}

Given,

$$
F=P \times r \times w
$$

where,

$\mathrm{F}=$ force in tension, $\mathrm{N}$

$\mathrm{P}=$ pressure, $\mathrm{Pa}$

$\mathrm{r}=$ radius of body, $\mathrm{m}$

$\mathrm{w}=$ width of the material, $\mathrm{m}$ 
as outlined previously, it is rather simple to solve for the force/width values given a desired pressure and the average radius for a given location of the leg. This average radius was based on the circumference measurement conducted in the previous phase. With the force/width data, the strain needed to properly pressurize the leg is easily calculated from the material testing in phase 1 . While the force is constant for each application, the strain depends on whether the design is a single wrap, double wrap, or triple wrap. With the needed strain solved for (based on the wrap design), the bands can be calibrated easily by solving the simple equation:

$$
x=\frac{\text { circumference }}{1+\varepsilon}
$$

In this equation, $\varepsilon$ is the strain, while $\mathrm{x}$ is the distance to be measured to cover that circumference. The value for $\mathrm{x}$ was divided into 8 parts evenly in order to create more markings to guide the test conductor, as seen in Figure 97. Every $8^{\text {th }}$ column, shown in that figure with little dots next to the calibration lines, is marked differently to ensure that the proper columns are aligned when the elastic band is wrapped on the leg.

The code used to calculate these values is located in the following section.

\subsection{Matlab code}

\subsubsection{Bottom-up and top-down code}

\subsubsection{1 start_file_band_determination.m}

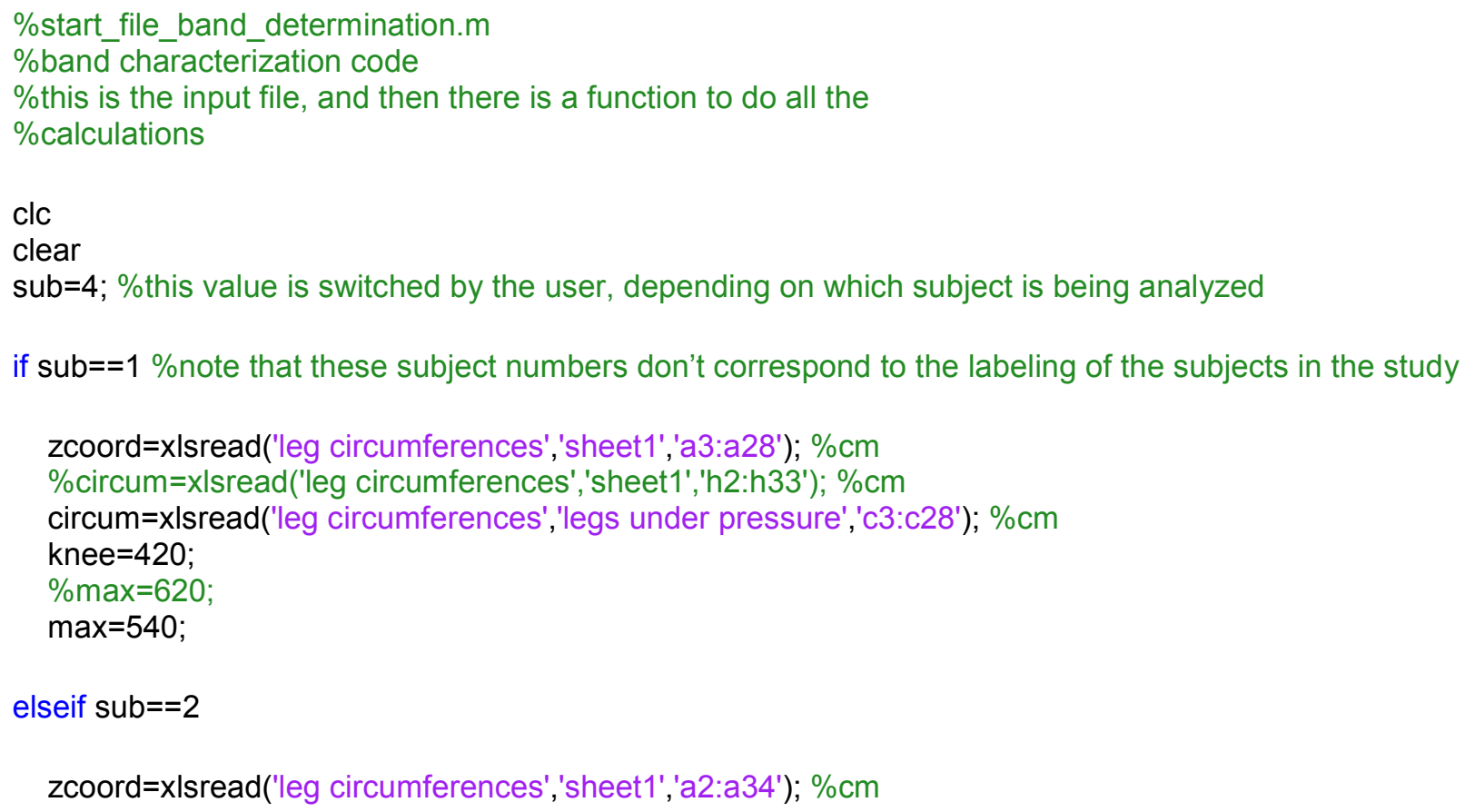




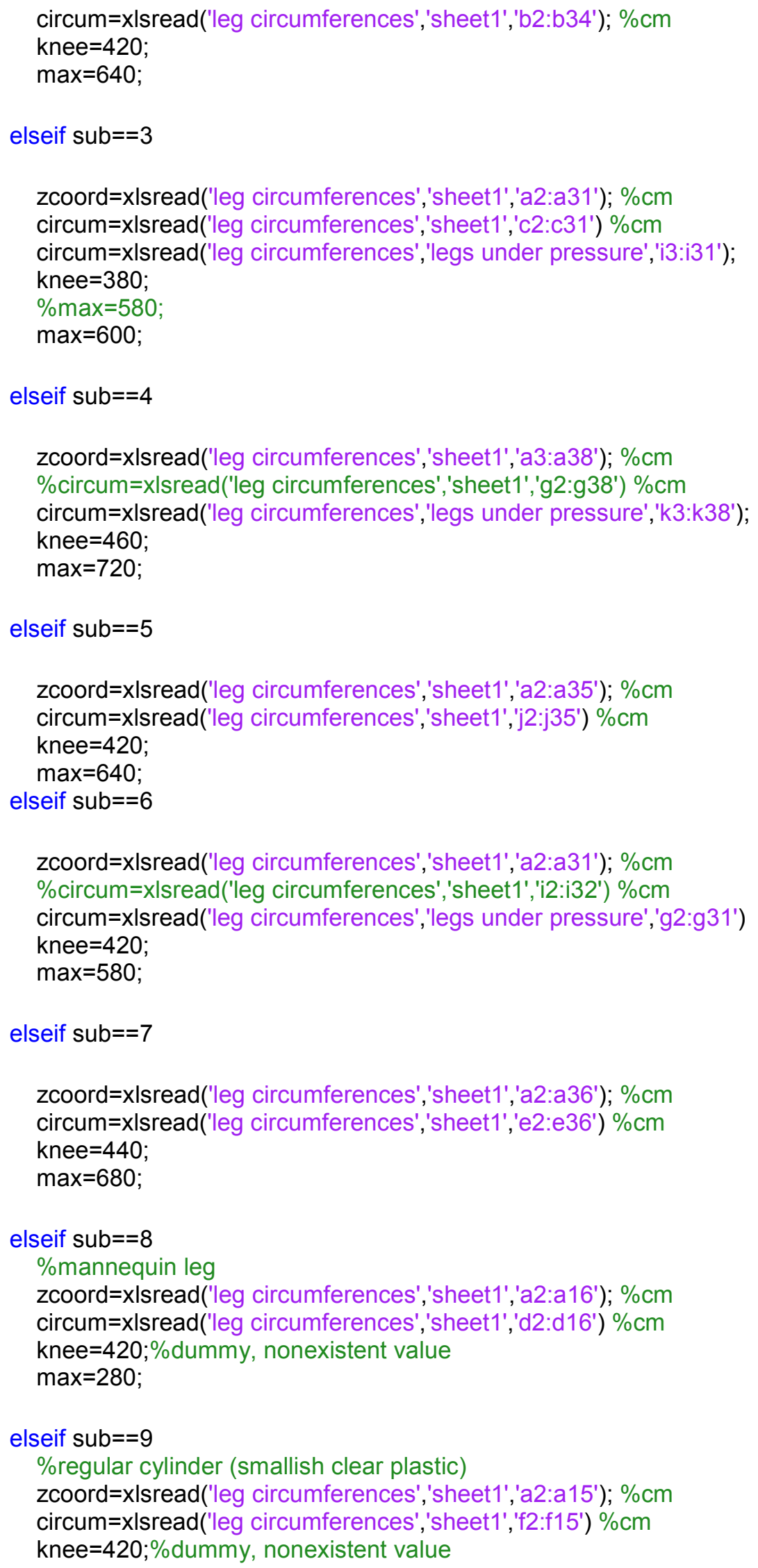


$\max =275$;

elseif sub $==10$

zcoord=xlsread('leg circumferences','sheet1','a2:a35'); \%cm

\%circum=xlsread('leg circumferences','sheet1','k2:k38') \%cm

\%normal circumference

circum=xlsread('leg circumferences','legs under pressure','e3:e35');

knee $=420 ; \%$ dummy, nonexistent value

$\max =680$;

end

\%everything in $\mathrm{mm}$

zcoord $=$ zcoord* 10 ;

circum $=$ circum ${ }^{*} 10$;

$\%\{$

This section is for the top-down wrap

circumlength=length(circum);

for counter=1:circumlength $\%$ this section of code reorients the leg for a top down wrap tempz(counter)=zcoord (circumlength+1-counter);

tempcircum(counter) $=$ circum (circumlength+1-counter);

end

zcoord=tempz

circum=tempcircum

\%end section for top-down wrap

$\%\}$

$\%$ regression coefficients where y axis=strain $(\%), x$ axis=FperW $(\mathrm{N} / \mathrm{mm})$

$x(1)=-65.103$; \%coefficient in front of cubic

$x(2)=153.45 ; \%$ square

$x(3)=-4.6184$; \%linear

$\%$ regression coefficients for plastically stretched bands

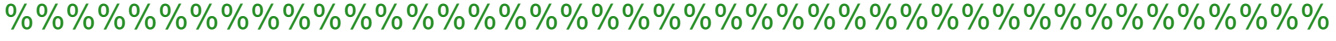

$\% \times(1)=-51.772$

$\% \times(2)=125.95$;

$\% \times(3)=29.776$;

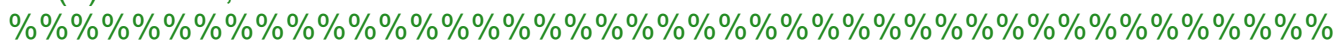

\%regression coefficients where y axis=width $(\mathrm{mm}), x$ axis=FperW $(\mathrm{N} / \mathrm{mm})$

$\mathrm{y}(1)=1.7195 ; \%$ square

$y(2)=-8.6982 ; \%$ linear

$y(3)=38.154 ; \%$ constant

$\%$ The following line is for the top down wrap

$\%[h, r$, strain, w,f,mark,wcircum] $=$ thigh_down_wrap(zcoord,circum, $x, y, k n e e, m a x)$;

[h,r,strain,w,f,mark,wcircum]=band_determine(zcoord,circum, $x, y$, knee,max);

$\%$ the large number of variables returned are in case the user wants to

$\%$ check any of the calculations, but they aren't necessary for the band

$\%$ determination 
\%the names that the data is written, can and should be changed

\%the "iterate" in the file name notes that the data being read is taken

\%from the subject's leg underpressure, and "DW"= double-wrap

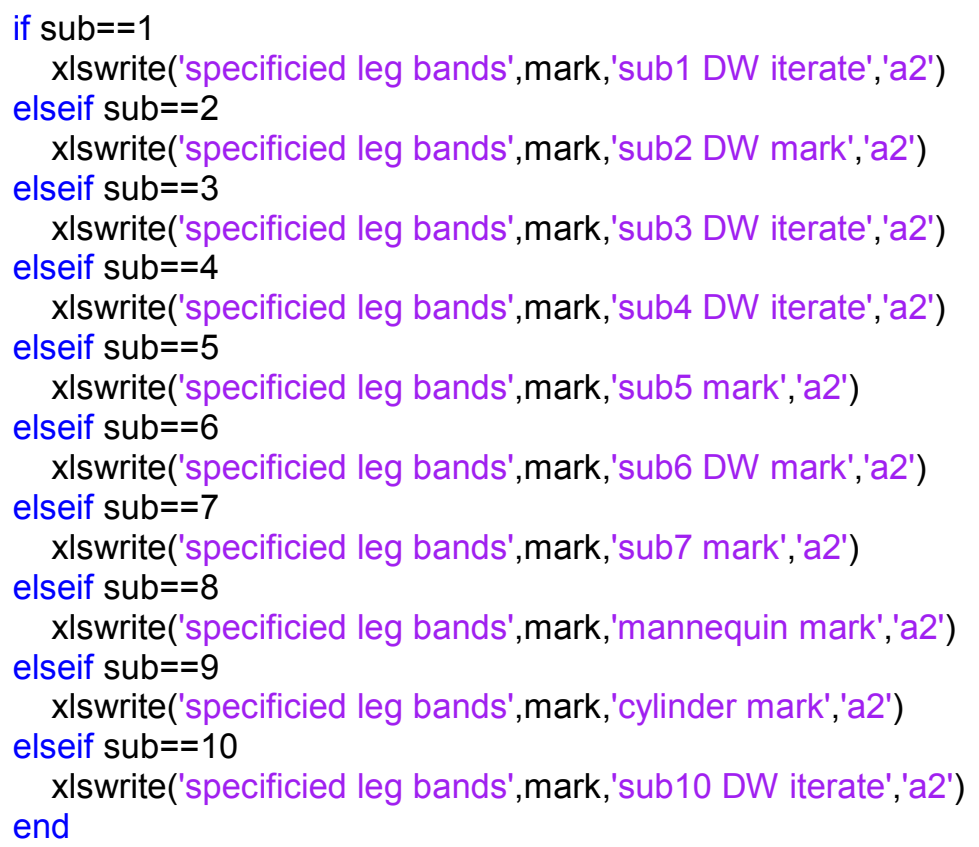

\subsubsection{2 band_determine.m}

function [h,r,strain,w,f,mark,wcircum]=band_determine(zcoord,circum, $x, y, k n e e$, max $)$

$\%$ This function actually does the calculations to produce the calibratied

$\%$ bands

$\%$ The following 2 lines need to be changed if a single wrap or double wrap

$\%$ or triple wrap is being used

pressure_perlayer=15;

$\% 7.5 \mathrm{kPa}$ is 2 double wraps (ie the cut and paste for the dummies)

$\% 15$ is a normal double wrap

$\% 30$ is a single wrap

$\% 10$ is a triple wrap

width_factor $=1 / 2 ; \% 1 / 3$ for triple wrap

$\% 1 / 2$ for double wrap

\%for single wrap change this to $5 \mathrm{~mm}$, and remove the necessary code as

$\%$ shown below, noted by the ${ }^{* * * * * *}$

$\mathrm{h}(1)=40 ; \%$ start $40 \mathrm{~mm}$ above ankle

$\mathrm{r}(1)=\operatorname{circum}(2) / 2 / \mathrm{pi}$;

$\%$ start at $4 \mathrm{~cm}$ above the ankle to calculate the radius based on the

$\%$ circumference

$\operatorname{mark}(1,1: 7)=0$;

$\operatorname{mark}(1,8)=\operatorname{circum}(2) / 10$; \% Note this outputs in $\mathrm{cm}$

$\%$ the first part of the wrap is to go over the leg, and then the second

$\%$ layer is actually used to create the pressure 


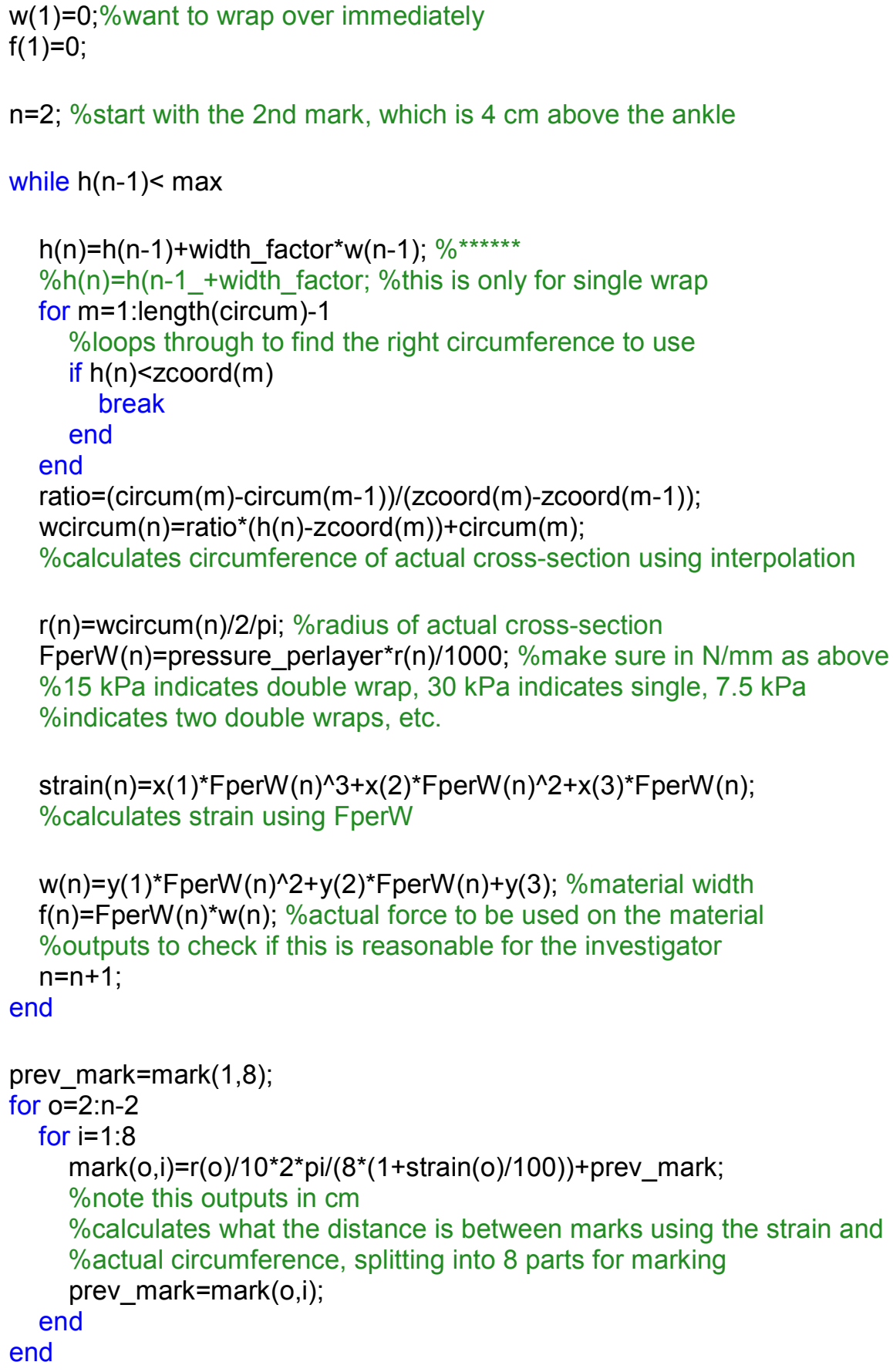

\subsubsection{3 thigh_down_wrap.m}

function [h,r,strain,w,f,mark,wcircum]=thigh_down_wrap(zcoord,circum,x,y,knee,max)

$\%$ This function actually does the calculations to produce the calibratied

$\%$ bands 
$\%$ The following 2 lines need to be changed if a single wrap or double wrap

$\%$ or triple wrap is being used

pressure_perlayer $=15$

$\% 7.5 \mathrm{kPa}$ is 2 double wraps (ie the cut and paste for the dummies)

$\% 15$ is a normal double wrap

$\% 30$ is a single wrap

$\% 10$ is a triple wrap

width_factor $=1 / 2 ; \% 1 / 3$ for triple wrap

$\% 1 / 2$ for double wrap

$\%$ for single wrap change this to $5 \mathrm{~mm}$, and remove the necessary code as

$\%$ shown below, noted by the ${ }^{* * * * *}$

$\mathrm{h}(1)=40 ; \%$ start $40 \mathrm{~mm}$ above ankle

$\mathrm{r}(1)=\operatorname{circum}(2) / 2 / \mathrm{pi}$;

$\%$ start at $4 \mathrm{~cm}$ above the ankle to calculate the radius based on the

$\%$ circumference

$\operatorname{mark}(1,1: 7)=0$;

mark $(1,8)=$ circum $(2) / 10$; \%Note this outputs in $\mathrm{cm}$

$\%$ the first part of the wrap is to go over the leg, and then the second

$\%$ layer is actually used to create the pressure

$w(1)=0 ; \%$ want to wrap over immediately

$f(1)=0$;

$n=2$;

while $h(n-1)>40 \%$ set at 40 to ensure we use circum (1) which is at $20 \mathrm{~mm}$, and the width of the material is slightly less than $20 \mathrm{~mm}$ too

$h(n)=h(n-1)$-width_factor ${ }^{*} w(n-1) ; \%$ here we are wrapping down ${ }^{* * * * * *}$

$\% h(n)=h(n-1)$-width_factor;

for $m=1$ :length(circum)-1 \%loops through to find the right circumference to use

if $h(n)>z \operatorname{coord}(m)$

break

end

end

$\% \mathrm{~m}$

ratio $=(\operatorname{circum}(m)-\operatorname{circum}(m-1)) /(z \operatorname{coord}(m)-z \operatorname{coord}(m-1))$;

wcircum $(n)=$ ratio $^{*}(h(n)$-zcoord $(m))+\operatorname{circum}(m) ; \%$ calculates circumference of actual cross-section using interpolation

$r(n)=$ wcircum $(n) / 2 / p i ; \%$ radius of actual cross-section

FperW $(n)=$ pressure_perlayer ${ }^{\star} r(n) / 1000 ; \%$ make sure in $N / m m$ as above

$\operatorname{strain}(n)=x(1)^{*} F \operatorname{perW}(n)^{\wedge} 3+x(2)^{*} F \operatorname{perW}(n)^{\wedge} 2+x(3)^{*} F \operatorname{perW}(n) ; \%$ calculates strain using FperW

$\mathrm{w}(\mathrm{n})=\mathrm{y}(1)^{\star} \mathrm{FperW}(\mathrm{n})^{\wedge} 2+\mathrm{y}(2)^{\star} \mathrm{FperW}(\mathrm{n})+\mathrm{y}(3) ; \%$ material width

$f(n)=F p e r W(n)^{*} w(n) ; \%$ actual force to be used on the material

$\%$ outputs to check if this is reasonable for the investigator

$n=n+1 ;$

end

prev_mark=mark $(1,8)$; 
for $o=2: n-2$
for $i=1: 8$

$\operatorname{mark}(0, i)=r(0) / 10^{*} 2^{*} \mathrm{pi} /\left(8^{*}(1+\operatorname{strain}(0) / 100)\right)+$ prev_mark; \%note this outputs in $\mathrm{cm}$ $\%$ calculates what the distance is between marks using the strain and

\%actual circumference, splitting into 8 parts for marking prev_mark=mark $(0, \mathrm{i})$;

end

end 


\section{Appendix J: Seal between atmosphere and pressure chamber}

The seal seen in 5.4.3: ILC Dover seal design is composed of the following materials.

- 2 Aluminum 6061 T6 metal rings (dimensions seen in the following figures)

- 48 4-40 screws

- 24 screws with length $3 / 8$ " are used to hold the harness

- 24 screws with length $1 / 2$ " are used to clamp the rings together

- an inextensible bladder, urethane coated nylon fabric, donated by ILC Dover

- fishing wire

- a red strap belt

- Dow Corning vacuum grease

- o-ring with ID of 9.25 " and OD of $97 / 16 "$

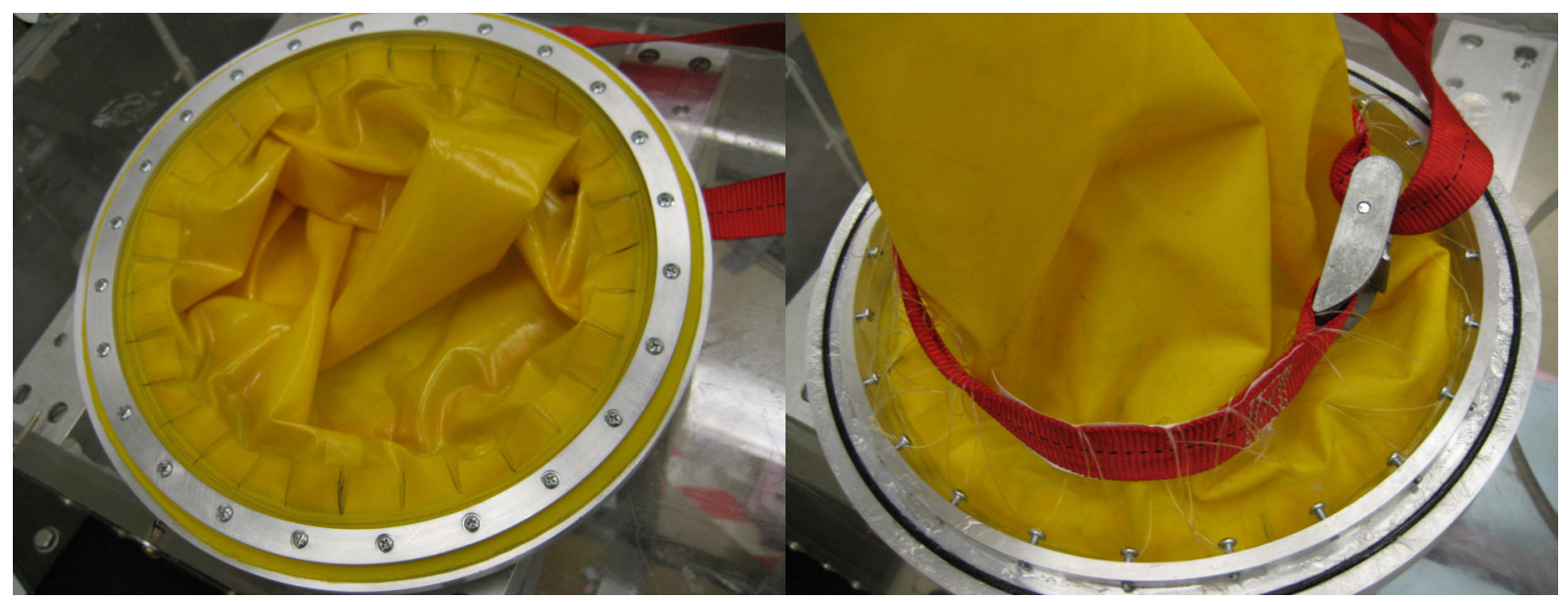

Figure 100: (left) Top view of ring Figure 101: (right) Bottom view of ring 


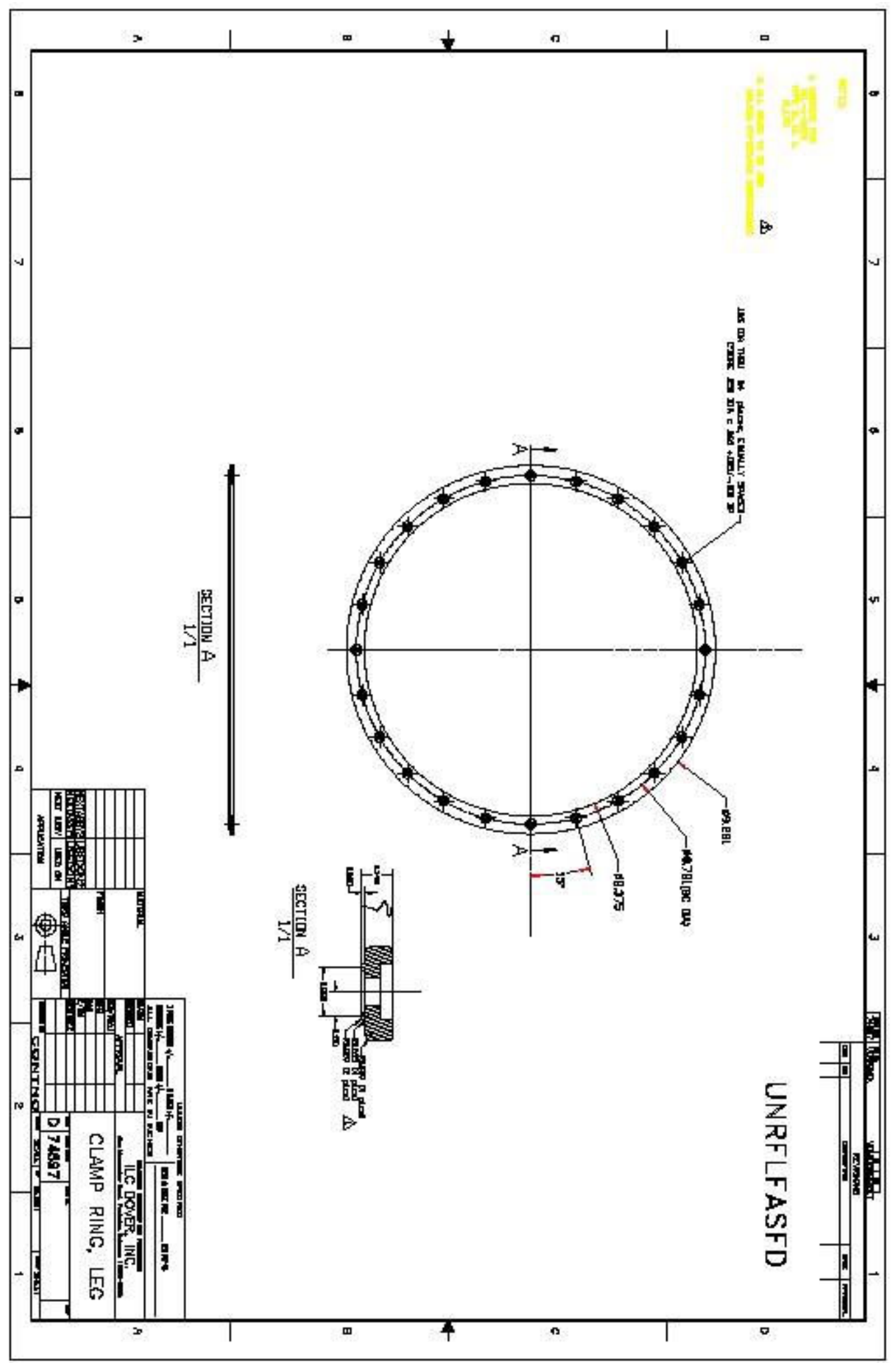

Figure 102: Top ring dimensions, given by ILC Dover 


$$
\begin{aligned}
& A=4.1875^{\prime \prime} \\
& B=4.625^{\prime \prime} \\
& C=4.71875^{\prime \prime} \\
& D=5^{\prime \prime} \\
& E=4.3125^{\prime \prime} \\
& F=4.3905^{\prime \prime}
\end{aligned}
$$

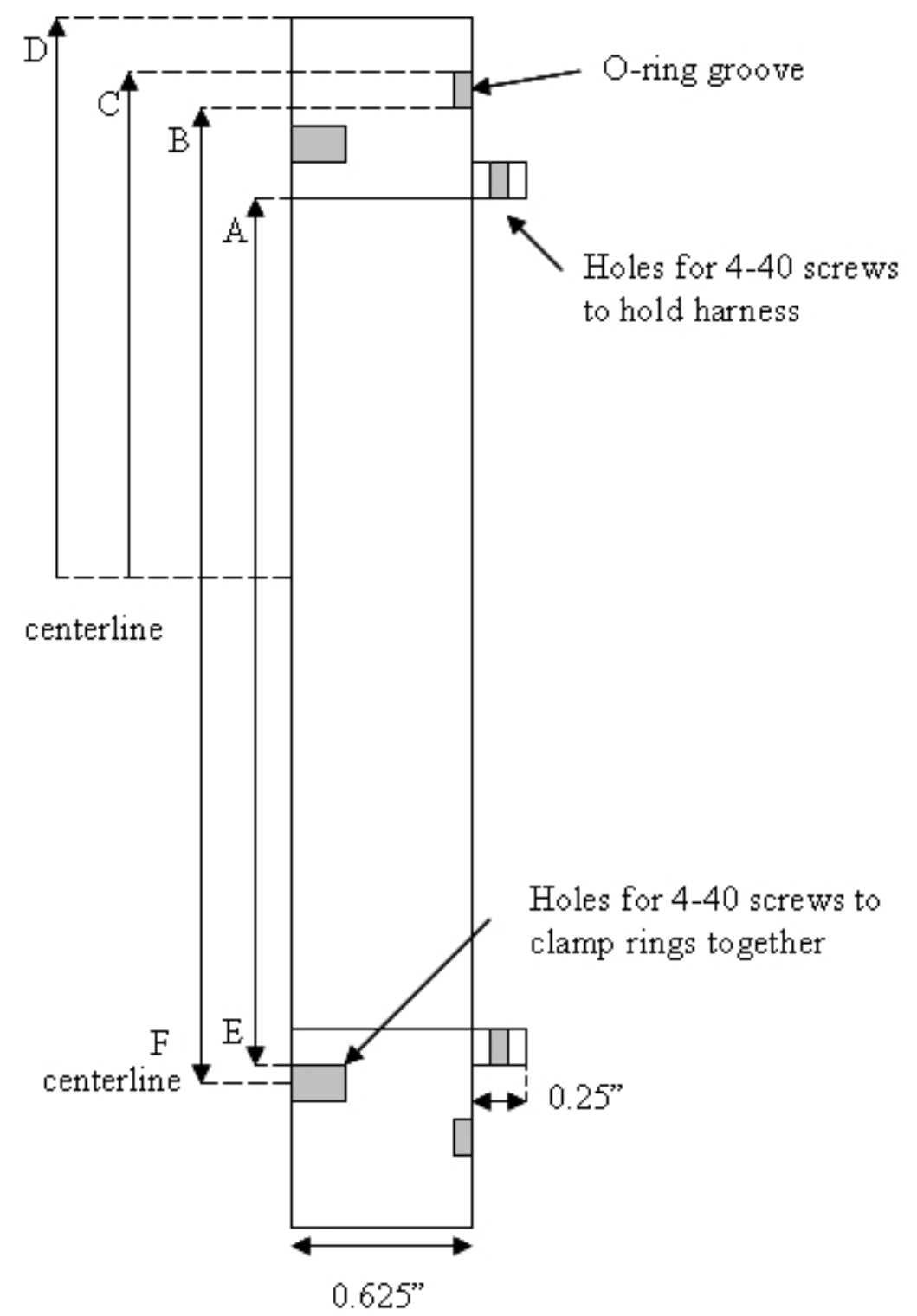

Figure 103: Bottom ring dimensions 


\section{Appendix K: Procedure for making socks}

\subsection{Materials to purchase}

- XL SealSkinz WaterBlocker ${ }^{\mathrm{TM}}$ socks

- 1 Bulkhead fitting (Part No. 5454K85, McMaster-Carr, New Brunswick NJ)

- Marine Goop Sealant (can be purchased at most local hardware stores, is made by Eclectic Products, Lineville, LA)

- Double-Bubble Urethane adhesive (Part No. 7493A21, McMaster-Carr)

- 2 o-rings with ID: 0.487" and OD: 0.693", can be either:

- (Part No. 9464K25, McMaster-Carr)

○ (Part No. 94115K112, McMaster-Carr)

\subsection{Procedure}

\subsubsection{Adding connection to atmosphere}

- mark spot you want to cut on one of the socks, which should be slightly below the blue line in the sock

- cut out a small triangle

- place in the bolt and washer

- put in nut and o-ring, then o-ring on outside, as shown in Figure 95

- use Double-Bubble urethane to seal over o-ring on outside of sock

- work into material, needs to get into the second layer, the rubber, in order to achieve a good seal

- let it dry

- repeat above 3 steps for o-ring on the inside of sock

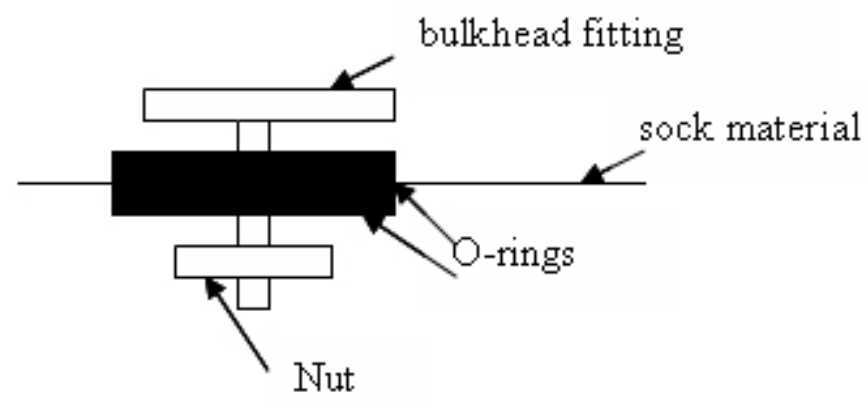

Figure 104: O-rings with bulkhead fitting on sock

\subsubsection{Sealing the 2 socks together}

- place the other sock (the one without the bulkhead fitting) inside-out inside the sock with the bulkhead fitting 
- stretch the socks over a cylinder, ensuring the socks are lined up evenly

$\circ$ the hard cylinder used in the TekScan calibration is a good option

- roll the top sock back up, so that the area on which the adhesive will be applied is visible

- want to glue the sections that are seen, try to get the very top of the white to white, which is the rubber and seems to hold together better

- Use marine goop to seal the socks together

- have 2 people roll down the top sock

- let dry for 24 hours or so

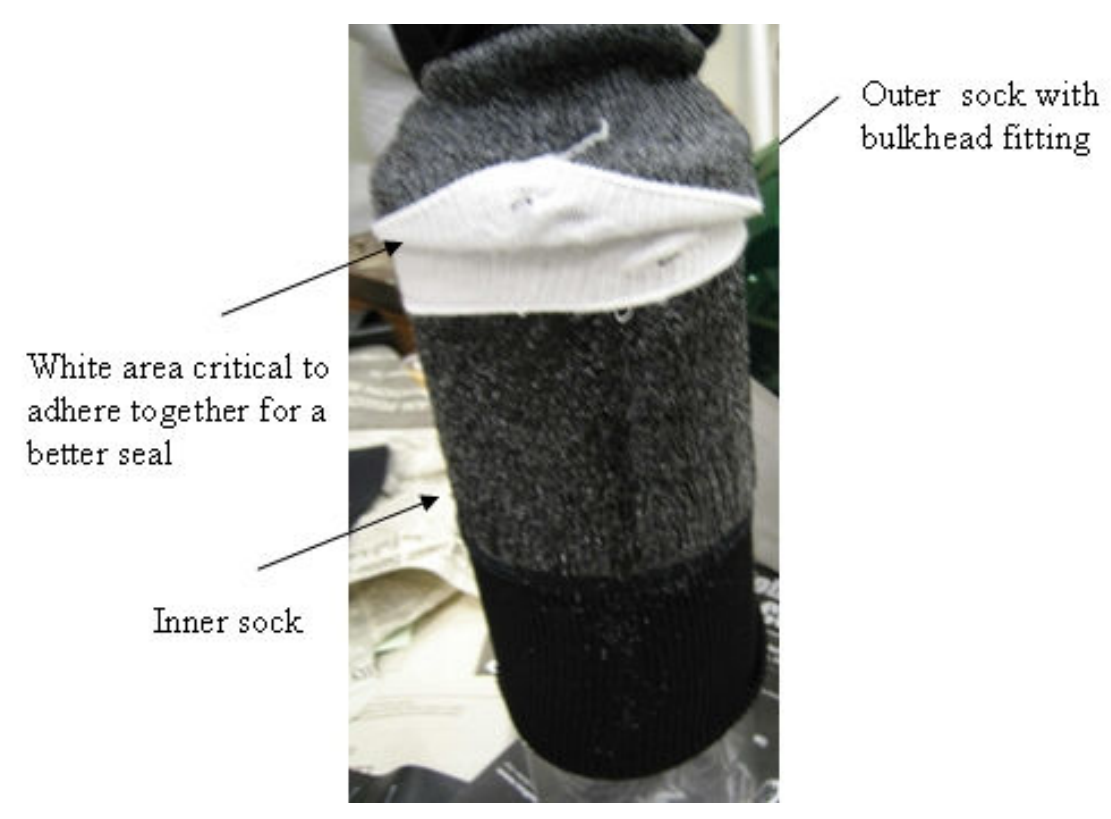

Figure 105: Procedure for sealing socks together 


\section{Appendix L: Experimental procedure}

\section{Overview}

6 minutes ambient, unsuited, control data.

Suited at $-225 \mathrm{mmHg}(-9$ ") for one hour, or until user / observer terminates test.

Then step up the pressure to room pressure and terminates test

\section{Well before test begins}

1. Calibrate Tekscan sensor.

\section{Subject preparation}

2. Ask subject if they need to use restroom.

3. Explain the test procedure to the subject, particularly discomfort scale

a. 0 normal atmospheric pressure

b. 1 feel somewhat abnormal, not really discomfort

c. 2 first sensations, mild discomfort, but tolerable

d. 3 some unpleasant sensations, increased discomfort

e. 4 significant discomfort,

f. 5 meaning finish the test

4. Put on seal and use to measure height in chamber. Ensure subject is wearing shoe while standing to get proper height.

5. Mark top level of seal on subject's leg to get the exact same spot afterwards

6. After chamber blocks adjustment, remove ring.

\section{Attach sensors}

7. Attach temperature sensors; thread sensor wires \#1-3 through seal.

a. \#1 on right shin, on bony part

b. \#2 on right thigh posterior (the back)

c. \#3 on right thigh anterior (the front)

d. Attach temp sensor \#4 on left thigh anterior (the front)

8. Attach BP cuff on left upper arm.

9. Check that all sensors are working.

a. close all other programs on computer except DataStudio and TekScan.

b. Set sampling rate to $0.2 \mathrm{~Hz}$ on watch AND temperature sensors (go to setup under temperature sensors, watch should be set automatically).

\section{Baseline measurements}

10. Make baseline physiological measurements in standing position for six minutes. Ring not required. Doesn't have to be in chamber.

11. Save the files in a new folder.

\section{Donning MCP bands}

12. Wrap leg with MCP band with help of one or more assistants.

a. place TekScan on leg 
b. place ring with seal on leg, ensuring TekScan and temp. sensors appropriately threaded through seal

c. Wrap 4-5 times just above ankle first.

d. Put on sock

e. Continue wrapping.

f. attach hose to sock

g. place on shoe

13. Plug in air hose for sock.

14. Have subject stand in chamber.

15. Plug in temperature and pressure sensors, Reattach sensor 4 (if necessary).

\section{Checklist}

16. Check the following

$\square$ Hose firmly attached to sock

$\square$ Temp. sensors attached to skin and won't fall off

$\square$ Sock is pushed down far enough

$\square$ No skin is exposed

$\square$ Tekscan is working

$\square$ Final wrap at top of leg is not too tight

$\square$ Sampling rate is set to $5 \mathrm{~s}$

$\square$ DataStudio file is saved with a name..

$\square$ Ensure sock is in shoe and firmly tied so pressure is maintained

17. Begin chamber pumpdown.

18. When chamber is at -9 ", start THREE timers simultaneously:
a. HR monitor
b. DataStudio
c. Stopwatch (as backup)

\section{Responsibilities}

\section{- Dan}

o monitor timing: every other minute, remind other test conductor to take BP

- monitor and control pump pressure

$\circ$ have access to emergency pump

- Record discomfort levels

$\circ$ TekScan measurements (every 5 minutes)

$\circ$ take photos whenever possible

\section{- UROP}

O Start temp sensor and HR monitor at the appropriate times

- Take BP

- Record $\mathrm{BP} / \mathrm{HR} /$ Temp every two minutes 
Name

Height

Weight

Test Date

Start time

PRE-TEST BASELINE MEASUREMENT

\begin{tabular}{|c|c|c|c|c|c|c|c|c|c|}
\hline $\begin{array}{l}\text { Time } \\
\text { (min) }\end{array}$ & $\begin{array}{c}\text { BP } \\
\text { Systolic }\end{array}$ & $\begin{array}{c}\text { BP } \\
\text { Diastolic }\end{array}$ & Discomfort & HR & $\begin{array}{c}\text { Temp } \\
1 \\
\left({ }^{\circ} \mathrm{C}\right)\end{array}$ & $\begin{array}{c}\text { Temp } \\
2 \\
\left({ }^{\circ} \mathrm{C}\right)\end{array}$ & $\begin{array}{c}\text { Temp } \\
\mathbf{3} \\
\left({ }^{\circ} \mathrm{C}\right)\end{array}$ & $\begin{array}{c}\text { Temp } \\
4 \\
\left({ }^{\circ} \mathrm{C}\right)\end{array}$ & $\begin{array}{c}\text { Issues / } \\
\text { comments } \\
\text { (note time } \\
\text { if } \\
\text { possible) }\end{array}$ \\
\hline 0 & & & & & & & & & \\
\hline 2 & & & & & & & & & \\
\hline 4 & & & & & & & & & \\
\hline 6 & & & & & & & & & \\
\hline
\end{tabular}

Day:

Day Indice:

Shin Length:

Thigh length: 
Name

\section{CHAMBER TEST}

First Hour - at -225mmHg

Pump down chamber to $-225 \mathrm{mmHg}(-9$ ”). When it reaches this pressure, begin timing.

\begin{tabular}{|c|c|c|c|c|c|c|c|c|c|}
\hline $\begin{array}{l}\text { Time } \\
\text { (min) }\end{array}$ & $\begin{array}{c}\text { BP } \\
\text { Systolic }\end{array}$ & $\begin{array}{c}\text { BP } \\
\text { Diastolic }\end{array}$ & Comfort & HR & $\begin{array}{c}\text { Temp } 1 \\
\left({ }^{\circ} \mathrm{C}\right)\end{array}$ & $\begin{array}{c}\text { Temp } 2 \\
\left({ }^{\circ} \mathrm{C}\right)\end{array}$ & $\begin{array}{c}\text { Temp } 3 \\
\left({ }^{\circ} \mathrm{C}\right)\end{array}$ & $\begin{array}{c}\text { Temp } 4 \\
\left({ }^{\circ} \mathrm{C}\right)\end{array}$ & $\begin{array}{l}\text { Issues / } \\
\text { comments }\end{array}$ \\
\hline \multicolumn{10}{|l|}{0} \\
\hline \multicolumn{10}{|l|}{2} \\
\hline \multicolumn{10}{|l|}{4} \\
\hline \multicolumn{10}{|l|}{6} \\
\hline \multicolumn{10}{|l|}{8} \\
\hline \multicolumn{10}{|l|}{10} \\
\hline \multicolumn{10}{|l|}{12} \\
\hline \multicolumn{10}{|l|}{14} \\
\hline \multicolumn{10}{|l|}{16} \\
\hline \multicolumn{10}{|l|}{18} \\
\hline \multicolumn{10}{|l|}{20} \\
\hline \multicolumn{10}{|l|}{22} \\
\hline \multicolumn{10}{|l|}{24} \\
\hline \multicolumn{10}{|l|}{26} \\
\hline \multicolumn{10}{|l|}{28} \\
\hline \multicolumn{10}{|l|}{30} \\
\hline \multicolumn{10}{|l|}{32} \\
\hline \multicolumn{10}{|l|}{34} \\
\hline \multicolumn{10}{|l|}{36} \\
\hline \multicolumn{10}{|l|}{38} \\
\hline \multicolumn{10}{|l|}{40} \\
\hline \multicolumn{10}{|l|}{42} \\
\hline \multicolumn{10}{|l|}{44} \\
\hline \multicolumn{10}{|l|}{46} \\
\hline \multicolumn{10}{|l|}{48} \\
\hline \multicolumn{10}{|l|}{50} \\
\hline \multicolumn{10}{|l|}{52} \\
\hline \multicolumn{10}{|l|}{54} \\
\hline 56 & & & & & & & & & \\
\hline 58 & & & & & & & & & \\
\hline 60 & & & & & & & & & \\
\hline
\end{tabular}

\section{NOTES}

FILENAMES

Time when test is terminated

Test was terminated by SUBJECTOTHER

OTHER NOTES / COMMENTS (write on back of sheet) 


\section{Appendix M: A more thorough investigation of Subject 4's test}

\subsection{Experimental data}

For the first 12 minutes (approximately) of the test in question, the subject experienced little discomfort, and the test was progressing normally. During this time the subject's heart rate was normal, if increasing slightly. (It started around $75 \mathrm{bpm}$, then rose to a maximum of $90 \mathrm{bpm}$.) See the figure below for more information. Part of this increase may have been due to an increase in conversation, explaining how she felt, to the test director, as during the first few minutes, during which time she did not talk. (Subjects are generally encouraged not to speak, as speaking is known to cause inaccuracy in blood pressure and pulse readings.) Please note that $t=0$ on the time scale indicates when the chamber has reached the desired negative underpressure, which in this case is $-225 \mathrm{~mm} \mathrm{Hg}$. There are no data points for the subject with the wrapped leg prior to this time period, since having the sensors attached to the subject plugged into the readout device while he or she entered the chamber could potentially cause the subject to trip over the cords and/or damage the equipment.

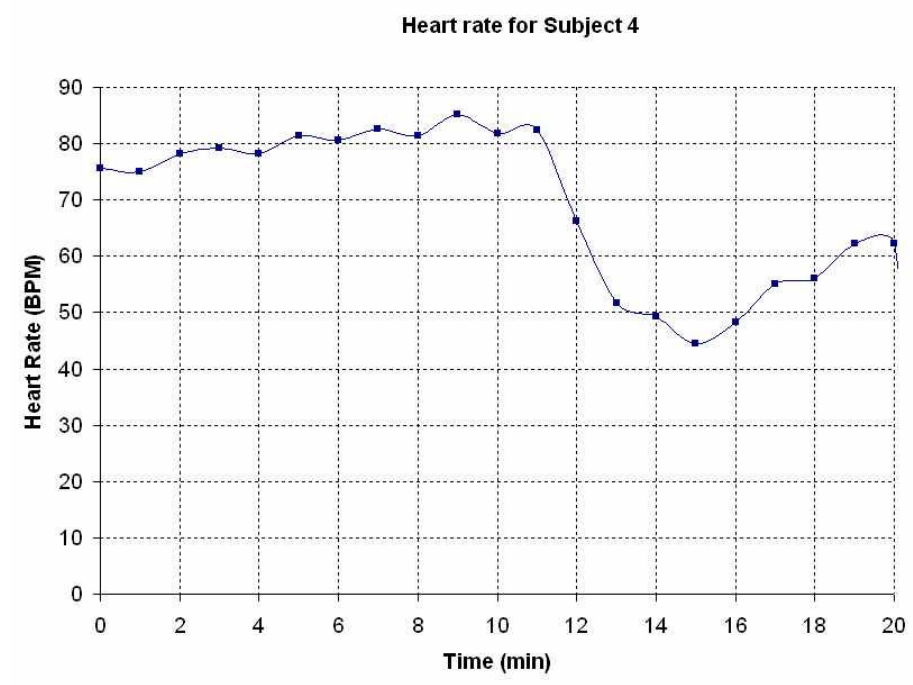

Figure 106: The heart rate of Subject 4

Please note that each of these points is the average over a minute starting at that time value, as data points were taken every 5 seconds. In other words, the data point at 12 minutes showing the heart rate decrease includes the data from 12 minutes to 12 minutes and 55 seconds.

During this time the subject's blood pressure was steady at 100/70 (systolic/diastolic), which was consistent with her readings under only atmospheric pressure. (Her blood pressure readings had been taken 3 separate times, once a few minutes before, and also on separate occasions 6-8 weeks previously. All readings were approximately 100/70, as can be seen in the table below). This low 
pressure (much lower than any other subject's) could perhaps increase her susceptibility to presyncope, similar to how fighter pilots in superior cardiovascular condition have more difficulty responding to the g-levels in their aircraft. Once again, 0 on the time scale represents when the chamber has been depressurized to the proper level. The readings, which take approximately 30-45 seconds, are taken starting at the time indicated. In other words, the reading at 0 minutes is based on data collected over the first 30-45 seconds of the experiment, with the first pumping of the gauge at 0 minutes.

Table 37: Subject 4's blood pressure data in the chamber

\begin{tabular}{|c|c|c|}
\hline $\begin{array}{l}\text { Time } \\
\text { (min) }\end{array}$ & $\begin{array}{c}\text { BP } \\
\text { (Systolic/Diastolic) }\end{array}$ & Comments \\
\hline 0 & $106 / 72$ & \\
\hline 2 & $151 / 68$ & $\begin{array}{l}\text { This systolic reading seems to be an anomaly, possibly } \\
\text { due to lack of accuracy in the BP measurement system. } \\
\text { The system has in other cases occasionally given the } \\
\text { test director no data at all, or impossible data (diastolic } \\
\text { values higher than systolic), so given the subject's lack } \\
\text { of discomfort at this stage of the experiment, it is } \\
\text { probable that this reading is inaccurate. }\end{array}$ \\
\hline 4 & $104 / 71$ & \\
\hline 6 & $101 / 68$ & \\
\hline 8 & $104 / 68$ & \\
\hline 10 & $99 / 62$ & \\
\hline 12 & $119 / 57$ & $\begin{array}{l}\text { The subject was talking during this time period, } \\
\text { possibly accounting for the raised systolic pressure and } \\
\text { decreased diastolic blood pressure. }\end{array}$ \\
\hline
\end{tabular}

The following table shows the subject's blood pressure readings taken on 3 separate occasions. Please note that the data in the last column was taken a few minutes prior to the adverse event. Some of the readings, such as the 16 given for the diastolic on January 11, 2007 at $t=0$ minutes are probably inaccurate, for reasons explained in the comment in the previous table at $\mathrm{t}=2$ minutes.

Table 38: Subject 4's blood pressure prior to chamber experiment

\begin{tabular}{|c|c|c|c|}
\hline & Nov. 1, 2006 & Nov.17, 2006 & Jan. 11, 2007 \\
\hline Time (min) & $\begin{array}{c}\text { BP } \\
\text { (Systolic/Diastolic) }\end{array}$ & $\begin{array}{c}\text { BP } \\
\text { (Systolic/Diastolic) }\end{array}$ & $\begin{array}{c}\text { BP } \\
\text { (Systolic/Diastolic) }\end{array}$ \\
\hline 0 & $137 / 60$ & $\begin{array}{c}101 /(\text { no data } \\
\text { available) }\end{array}$ & $95 / 16$ \\
\hline 2 & $101 / 69$ & $106 / 71$ & $95 / 67$ \\
\hline 4 & $101 / 76$ & $106 / 70$ & $95 / 64$ \\
\hline 6 & $\mathrm{n} / \mathrm{a}$ & $107 / 70$ & $92 / 67$ \\
\hline
\end{tabular}

At approximately 13 minutes, the subject stated that she did not think she could continue. The test director asked her if she was sure she wished to stop, to ensure that the experiment was not ended 
prematurely. A brief examination of the subject revealed her lips to be bluish and her overall face to be somewhat pale. The subject indicated that she indeed wished to end the experiment. This whole process took no more than 10 seconds. Immediately, the test director opened the pressure relief valve, as specified by the COUHES directions, and returned the chamber to atmospheric pressure.

\subsection{Subject treatment}

After the subject exited the pressure chamber, she wished to lay down on the top of the chamber. The test director and assistant helped her remove her leg from the chamber, and worked to rapidly unwrap her leg, so that her body was experiencing only atmospheric pressure at this point. The team members maintained a constant dialogue with the subject to ensure that there was no serious problem with her.

After approximately 3-5 minutes of lying on the chamber, the subject sat up and was helped to a chair a few feet away. In this relatively small time period she improved dramatically: her face regained color, and her heart rate started returning to normal (see figure in previous section). The test director began to speak with her regarding her experience, to understand why the adverse event might have occurred. Upon questioning, she stated that she had had enough sleep and food that day, and had not given blood recently. She again stated that throughout the first 12 minutes she had not felt particularly uncomfortable, and had only suddenly felt so poorly. She also informed the test director that when he had pulled the pressure release valve she had not been able to see him, despite the fact that he was standing less that a foot to her right, and easily within her normal field of vision. This was one of the indications of presycope mentioned above.

As the subject became more relaxed and recovered more fully, the test director inspected her legs, per the protocol, in order to determine if she had any edema. Very little edema was seen, as shown in the following figure.

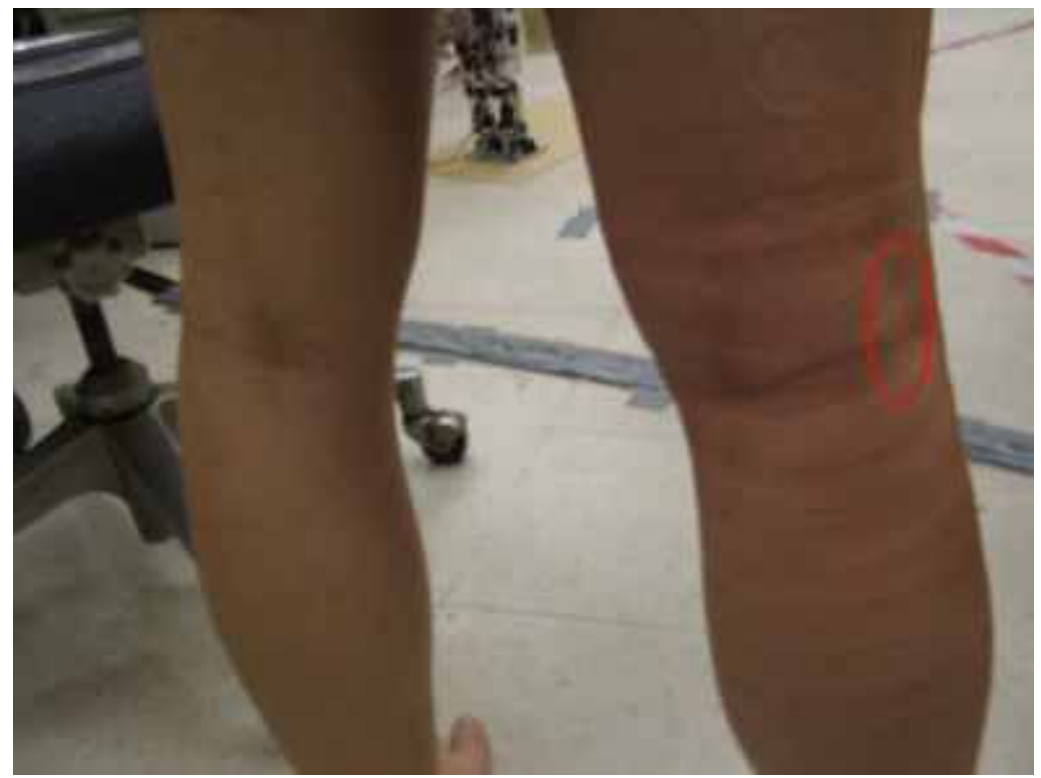

Figure 107: Subject 4's leg after recovery 
While some indentations from the band are shown, there is very little evidence of edema, other than a minimal amount on the posterior of the knee. The right leg looks significantly larger than the left, but that is due to a poor choice of perspective for the photo, not because the right leg had experienced ill effects from the time in the chamber.

After approximately 10 minutes of additional time sitting in the chair, the subject wished to leave the experimental area. As the subject seemed fully recovered at that point, the test director saw no reason to disagree with this reasonable request. He did, however, suggest that she visit MIT Medical either at this time, or if she subsequently felt any further symptoms. She did not feel that this was necessary. The test director made certain that the subject would not be alone after leaving the experimental area, in order to ensure her safety. He also went back to check on her about an hour later, at which point she declared herself fully recovered. While the subject was not fitted with any of the sensors to take quantitative measurements to support this statement, the return of her lips and face to their normal colors supported her assertion. The test director has spoken with the subject on a near-daily basis since the experiment, and she has reported no further symptoms from her participation in the experiment.

The subject was excused from any further involvement in the experiment. 


\section{Appendix N: Matlab code to calculate underpressure felt by subjects in the pilot study}

\subsection{Matlab files}

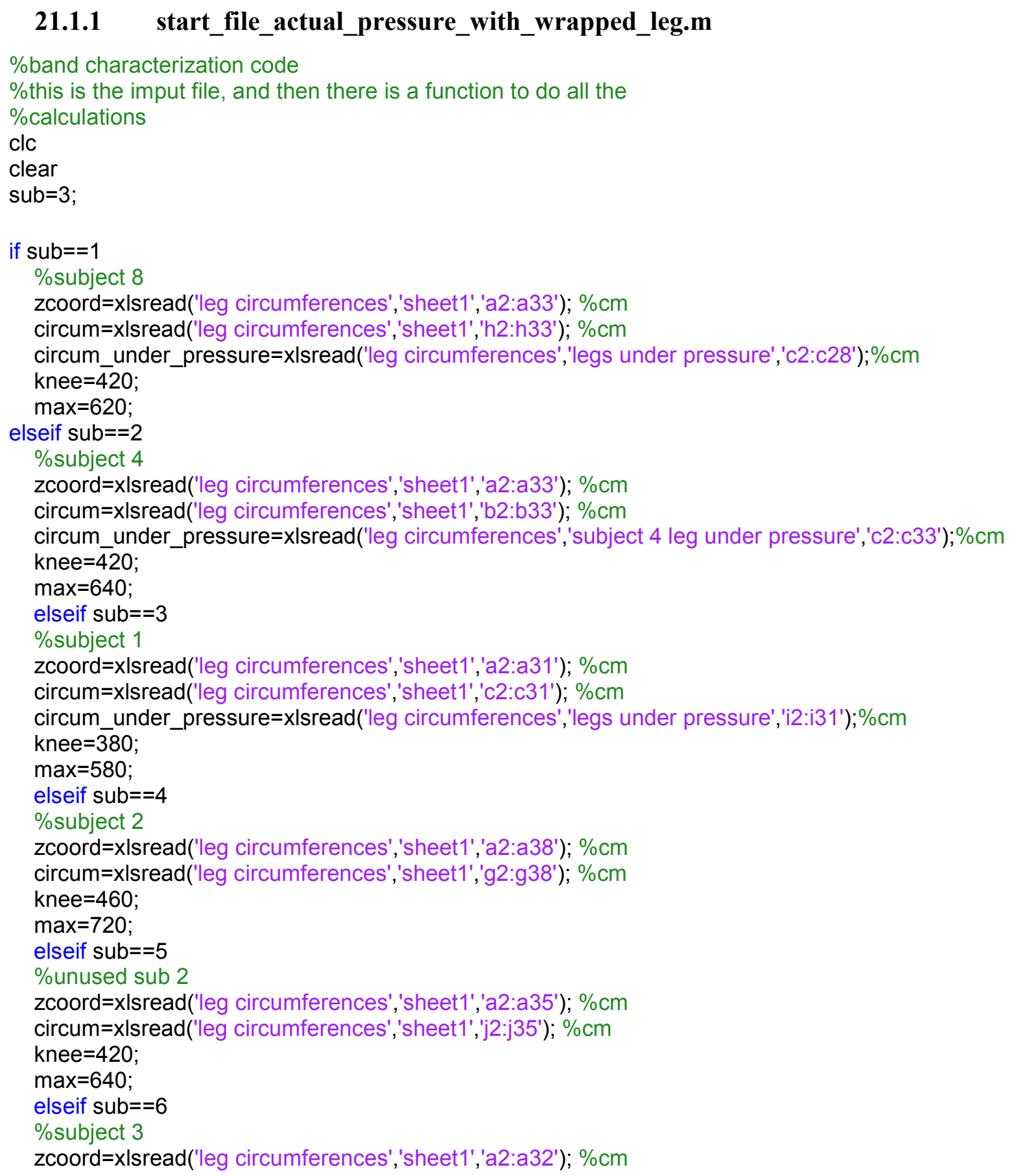


circum=xlsread('leg circumferences','sheet1','i2:i32'); \%cm

circum_under_pressure=xlsread('leg circumferences','legs under pressure','g2:g31');\%cm knee $=\overline{420}$;

$\max =580$

elseif sub==7

\%unused sub 1

zcoord=xlsread('leg circumferences','sheet1','a2:a36'); \%cm

circum=xlsread('leg circumferences','sheet1','e2:e36') ;\%cm

knee $=440$;

$\max =680$;

elseif sub $==8$

$\%$ mannequin leg

zcoord=xlsread('leg circumferences','sheet1','a2:a16'); \%cm

circum=xlsread('leg circumferences','sheet1','d2:d16'); \%cm

knee $=420 ; \%$ dummy, nonexistent value

$\max =280$;

elseif $\mathrm{sub}==9$

\%regular cylinder (smallish clear plastic)

zcoord=xlsread('leg circumferences','sheet1','a2:a15'); \%cm

circum=xlsread('leg circumferences','sheet1','f2:f15'); \%cm

knee $=420 ; \%$ dummy, nonexistent value

$\max =275$;

elseif $\mathrm{sub}==10$

$\%$ subject 6

zcoord=xlsread('leg circumferences','sheet1','a2:a38'); \%cm

circum=xlsread('leg circumferences','sheet1','k2:k38');\%cm

circum_under_pressure=xlsread('leg circumferences','legs under pressure','e2:e35'); \%cm

knee $=\overline{420} \%$ dummy, nonexistent value

$\max =720$;

end

length(circum);

\%everything in $\mathrm{mm}$

zcoord $=$ zcoord ${ }^{*} 10$;

circum $=$ circum $* 10$;

circum_under_pressure $=$ circum_under_pressure ${ }^{*} 10$;

$\%$ regression coefficients where y axis=strain $(\%), x$ axis=FperW $(\mathrm{N} / \mathrm{mm})$

$x(1)=-65.103 ; \%$ coefficient in front of cubic

$x(2)=153.45 ; \%$ square

$x(3)=-4.6184$; \%linear

\%regression coefficients where y axis=width $(\mathrm{mm}), x$ axis=FperW $(\mathrm{N} / \mathrm{mm})$

$y(1)=1.7195 ; \%$ square

$y(2)=-8.6982 ; \%$ linear

$y(3)=38.154 ; \%$ constant

$\%$ reverse coefficients, where y axis $=$ FperW $(\mathrm{N} / \mathrm{mm})$ and $\mathrm{x}$ axis $=$ strain $(\%)$

$z(1)=1.97^{*} 10^{\wedge}-6$;

$z(2)=-3.56^{*} 10^{\wedge}-4$;

$z(3)=2.74^{*} 10^{\wedge}-2$; 
[strain,strain_under_pressure,FperW,actual_FperW,theory_pressure,actual_pressure]=actual_pressure_with _wrapped_leg(zcoord,circum,x,y,circum_under_pressure,max,z);

if sub==1

xlswrite('actual pressure with leg bands.xls',zcoord,'subject 8','a3')

xlswrite('actual pressure with leg bands.xls',circum,'subject 8','b3')

xlswrite('actual pressure with leg bands',strain','subject 8','c3')

xlswrite('actual pressure with leg bands',FperW','subject 8','d3')

xlswrite('actual pressure with leg bands',theory_pressure','subject 8','e3')

xlswrite('actual pressure with leg bands',circum_under_pressure,'subject 8','h3')

xlswrite('actual pressure with leg bands',strain_under_pressure','subject 8','i3')

xlswrite('actual pressure with leg bands',actual_FperW','subject 8','j3')

xlswrite('actual pressure with leg bands',actual_pressure','subject 8','k3')

elseif sub $==2$

xlswrite('actual pressure with leg bands.xls',zcoord,'subject 4','a3')

xlswrite('actual pressure with leg bands.xls',circum,'subject 4','b3')

xlswrite('actual pressure with leg bands',strain','subject 4','c3')

xlswrite('actual pressure with leg bands',FperW','subject 4','d3')

xlswrite('actual pressure with leg bands',theory_pressure','subject 4','e3')

xlswrite('actual pressure with leg bands',circum_under_pressure,'subject 4','h3')

xlswrite('actual pressure with leg bands',strain_under_pressure','subject 4','i3')

xlswrite('actual pressure with leg bands',actual_FperW','subject 4','j3')

xlswrite('actual pressure with leg bands',actual_pressure','subject 4','k3')

elseif $\mathrm{sub}==3$

xlswrite('actual pressure with leg bands.xls',zcoord,'subject 1','a3')

xlswrite('actual pressure with leg bands.xls',circum,'subject 1','b3')

xlswrite('actual pressure with leg bands',strain','subject 1','c3')

xlswrite('actual pressure with leg bands',FperW','subject 1','d3')

xlswrite('actual pressure with leg bands',theory_pressure','subject 1','e3')

xlswrite('actual pressure with leg bands',circum_under_pressure,'subject 1','h3')

xlswrite('actual pressure with leg bands',strain_under_pressure','subject 1','i3')

xlswrite('actual pressure with leg bands',actual_FperW','subject 1','j3')

xlswrite('actual pressure with leg bands',actual_pressure','subject 1','k3')

elseif $s u b==6$

xlswrite('actual pressure with leg bands.xls',zcoord,'subject 3','a3')

xlswrite('actual pressure with leg bands.xls',circum,'subject 3','b3')

xlswrite('actual pressure with leg bands',strain','subject 3','c3')

xlswrite('actual pressure with leg bands',FperW','subject 3','d3')

xlswrite('actual pressure with leg bands',theory_pressure','subject 3','e3')

xlswrite('actual pressure with leg bands',circum_under_pressure,'subject 3','h3')

xlswrite('actual pressure with leg bands',strain_under_pressure','subject 3','i3')

xlswrite('actual pressure with leg bands',actual_FperW','subject 3','j3')

xlswrite('actual pressure with leg bands',actual_pressure','subject 3','k3')

elseif $\mathrm{sub}==10$

xlswrite('actual pressure with leg bands.xls',zcoord,'subject 6','a3')

xlswrite('actual pressure with leg bands.xIs',circum,'subject 6','b3')

xlswrite('actual pressure with leg bands',strain','subject 6','c3')

xlswrite('actual pressure with leg bands',FperW','subject 6','d3')

xlswrite('actual pressure with leg bands',theory_pressure','subject 6','e3')

xlswrite('actual pressure with leg bands',circum_under_pressure,'subject 6','h3')

xlswrite('actual pressure with leg bands',strain_under_pressure','subject 6','i3')

xlswrite('actual pressure with leg bands',actual_FperW','subject 6','j3') 
xlswrite('actual pressure with leg bands',actual_pressure','subject 6','k3')

end

\subsection{2 actual_pressure_with_wrapped_leg.m}

function

[strain,strain_under_pressure,FperW,actual_FperW,theory_pressure,actual_pressure]=actual_pressure_with _wrapped_leg(zcoord,circum,x,y,circum_under_pressure,max,z)

pressure_perlayer=15; $\% 7.5 \mathrm{kPa}$ is 2 double wraps (ie the cut and paste for the dummies)

$\% 15$ is a normal double wrap

$\% 30$ is a single wrap

$n=1$;

for $m=1$ :length(circum_under_pressure)

$r(n)=\operatorname{circum}(m) / 2 / p i ; \%$ radius of actual cross-section

FperW(n)=pressure_perlayer ${ }^{*} r(n) / 1000 ; \%$ make sure in $\mathrm{N} / \mathrm{mm}$ as above

$\% 15 \mathrm{kPa}$ indicates double wrap, $30 \mathrm{kPa}$ indicates single, $7.5 \mathrm{kPa}$

$\%$ indicates two double wraps, etc.

$\operatorname{strain}(n)=x(1)^{\star} F \operatorname{perW}(n)^{\wedge} 3+x(2)^{\star} F \operatorname{perW}(n)^{\wedge} 2+x(3)^{\star} F \operatorname{FerW}(n) ; \%$ calculates strain using FperW

$w(n)=y(1)^{*} F \operatorname{perW}(n)^{\wedge} 2+y(2)^{*} F \operatorname{perW}(n)+y(3) ; \%$ material width

$f(n)=F p e r W(n)^{*} w(n) ; \%$ actual force to be used on the material

$\%$ outputs to check if this is reasonable for the investigator

theory_pressure $(n)=F p e r W(n) / r(n) * 1000$;

r_under_pressure $(n)=$ circum_under_pressure $(m) / 2 / p i$;

strain_under_pressure(n)=r_under_pressure $(\mathrm{n}) / \mathrm{r}(\mathrm{n})^{*}(1+\operatorname{strain}(\mathrm{n}) / 100)-1 ; \%$ need to divide by 100 in order

to get proper dimensions

strain_under_pressure(n)=strain_under_pressure(n)*100; \%get back to percentage here

actual_FperW $(n)=z(1)^{*}$ strain_under_pressure $(n)^{\wedge} 3+z(2)^{*}$ strain_under_pressure $(n)^{\wedge} 2+z(3)^{*}$ strain_under_pres sure $(\mathrm{n})$;

actual_pressure(n)=1000/r_under_pressure(n)*actual_FperW(n); \%100 is for coversion to get to $\mathrm{kPa}$

$\mathrm{n}=\mathrm{n}+1$

end 


\section{Appendix O: A more thorough investigation of Subject 7's test}

\subsection{Experimental data}

For the first 42 minutes (approximately) of the test in question, the subject experienced little discomfort, and the test was progressing normally. During this time the subject's heart rate was fairly constant, although somewhat higher than an average person. See the figure below for more information. Please note that $\mathrm{t}=0$ on the time scale indicates when the chamber has reached the desired negative underpressure, $-225 \mathrm{~mm} \mathrm{Hg}$. There are no data points for the subject under pressure from the neoprene bands prior to this time period, since having the sensors attached to the subject plugged into the readout device while he or she entered the chamber could potentially cause the subject to trip over the cords and/or damage the equipment.

\section{Heart Rate for Subject 7 in chamber}

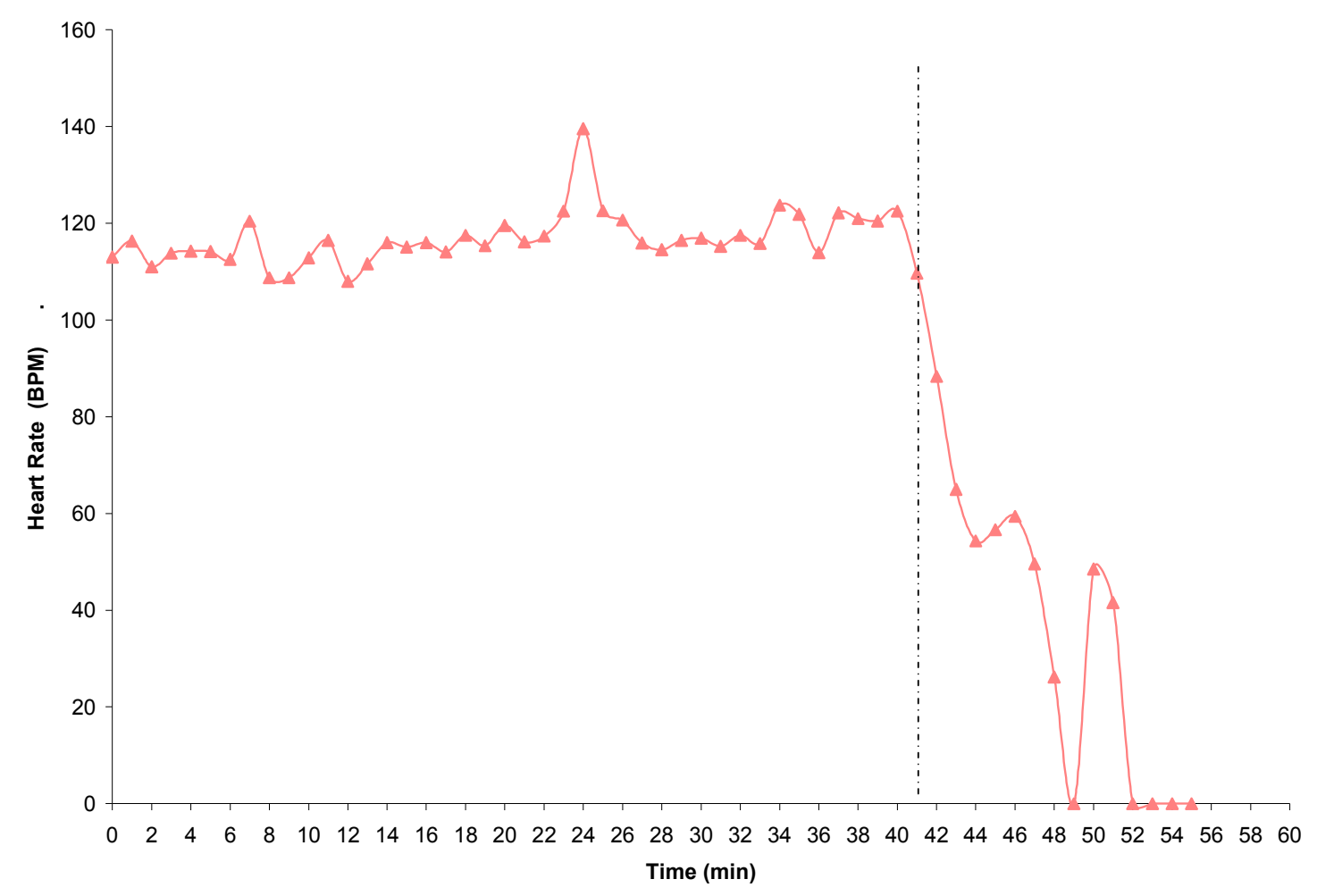

Figure 108: The heart rate of Subject 7

Please note that each of these points is the average over a minute starting at that time value, as data points were taken every 5 seconds. In other words, the data point at 42 minutes showing the heart 
rate decrease includes the data from 42 minutes to 42 minutes and 55 seconds. The dashed line indicates the approximate beginning of the adverse event. When the heart rate reaches 0 , the sensor simply stopped recording data. The lowest heart rate recorded was actually $53 \mathrm{bpm}$. The reason that the data points exist below that level is because those data points are the averages of the heart rate over the given minutes, as explained above.

During this time the subject's blood pressure experienced some variation. The team noted this variation with some skepticism for multiple reasons. First, the variation may be due to the lack of accuracy in the BP measurement system. The system has in other cases occasionally given the test director no data at all, or impossible data (diastolic values higher than systolic), so given the subject's lack of discomfort at this stage of the experiment, it is probable that this reading is inaccurate.

Once again, 0 on the time scale represents when the chamber has been depressurized to the proper level of $-225 \mathrm{~mm} \mathrm{Hg}$. The readings, which take approximately 30-45 seconds, are taken starting at the time indicated. In other words, the reading at 0 minutes is based on data collected over the first 30-45 seconds of the experiment, with the first pumping of the gauge at 0 minutes. For those times when no reading was available, the data was interpolated from the surrounding data points. The gray lines represent the averages of the baseline readings (without the third reading, which was considered inaccurate due to the large difference from the other readings) taken prior to donning the prototype and entering the chamber.

Blood Pressure for Subject 7 in chamber

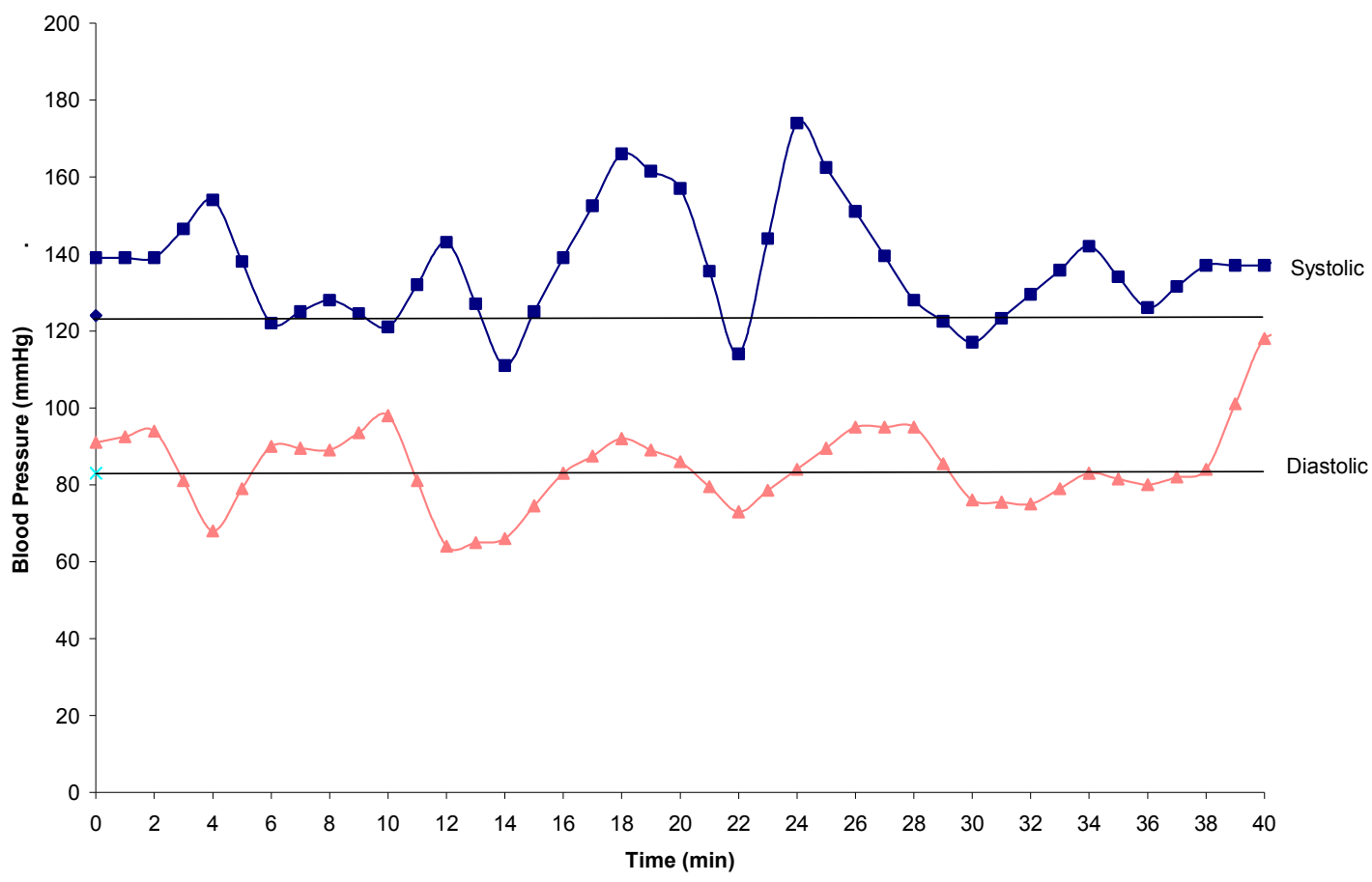

Figure 109: Blood pressure for Subject 7 
The following table shows the subject's blood pressure readings prior to the chamber entrance. The third reading (starting at 4 minutes) was removed from the baseline measurements above because it was considered inaccurate due to the difference from the other readings. As noted above, the blood pressure cuff provides impossible data, so the results are somewhat suspect, and therefore, the data is typically edited for what is thought to be accurate.

Table 39: Subject 7's blood pressure prior to chamber experiment

\begin{tabular}{|c|c|c|}
\hline Time (min) & Systolic & Diastolic \\
\hline 0 & 125 & 82 \\
\hline 2 & 127 & 81 \\
\hline 4 & $\mathrm{n} / \mathrm{a}$ & $\mathrm{n} / \mathrm{a}$ \\
\hline 6 & 120 & 86 \\
\hline
\end{tabular}

At approximately 40 minutes, the subject stated that she felt some nausea. The test director asked her if she wished to stop, but the subject replied that she wished to continue. The test director considered ending the test despite the subject's statement, since nausea is a symptom of presyncope, but decided to follow the subject's wishes. The reason for this decision, besides the stated desire of the subject, was that the previous subject who experienced the adverse event had not felt any nausea, while a few other subjects had felt some slight nausea, but were able to complete the test successfully without experiencing any adverse events. This subject had spent 30 more minutes in the chamber than the subject in the previous adverse event, so it was thought that this symptom was minor, and not a precursor to presyncope. It should also be noted that on a scale of $0-5$, with 5 being the most uncomfortable, the subject never stated that she felt worse than a 2 , which indicates mild, but tolerable discomfort.

A minute or two later, the subject stated that she felt hot. When queried to clarify, she stated that she felt hot over her entire body. At this point, it was clear that an adverse event was occurring, and the test was ended immediately, as the test director opened the pressure relief valve, as specified by the COUHES directions, and returned the chamber to atmospheric pressure.

In the previous adverse event, a rise of $3.4{ }^{\circ} \mathrm{C}$ increase on the anterior of the subject's thigh was thought to be an indicator of presyncope. However, the current subject experienced no such large change, with the biggest change in skin temperature measured on the front of the thigh (sensor 3), which rose $1.9^{\circ} \mathrm{C}$. This value is well within the range of other subjects who experienced no significant discomfort in the chamber, and as can be seen in the figure below, her skin temperature actually decreased slightly on the front of the thigh in the minutes before the adverse event occurred. The sensor on the left leg outside the chamber (sensor 4) shows a small decline throughout the time period, as do sensor 1 (shin anterior) and sensor 2 (thigh posterior). Please note that the scale on the $y$-axis begins at $27^{\circ} \mathrm{C}$. 


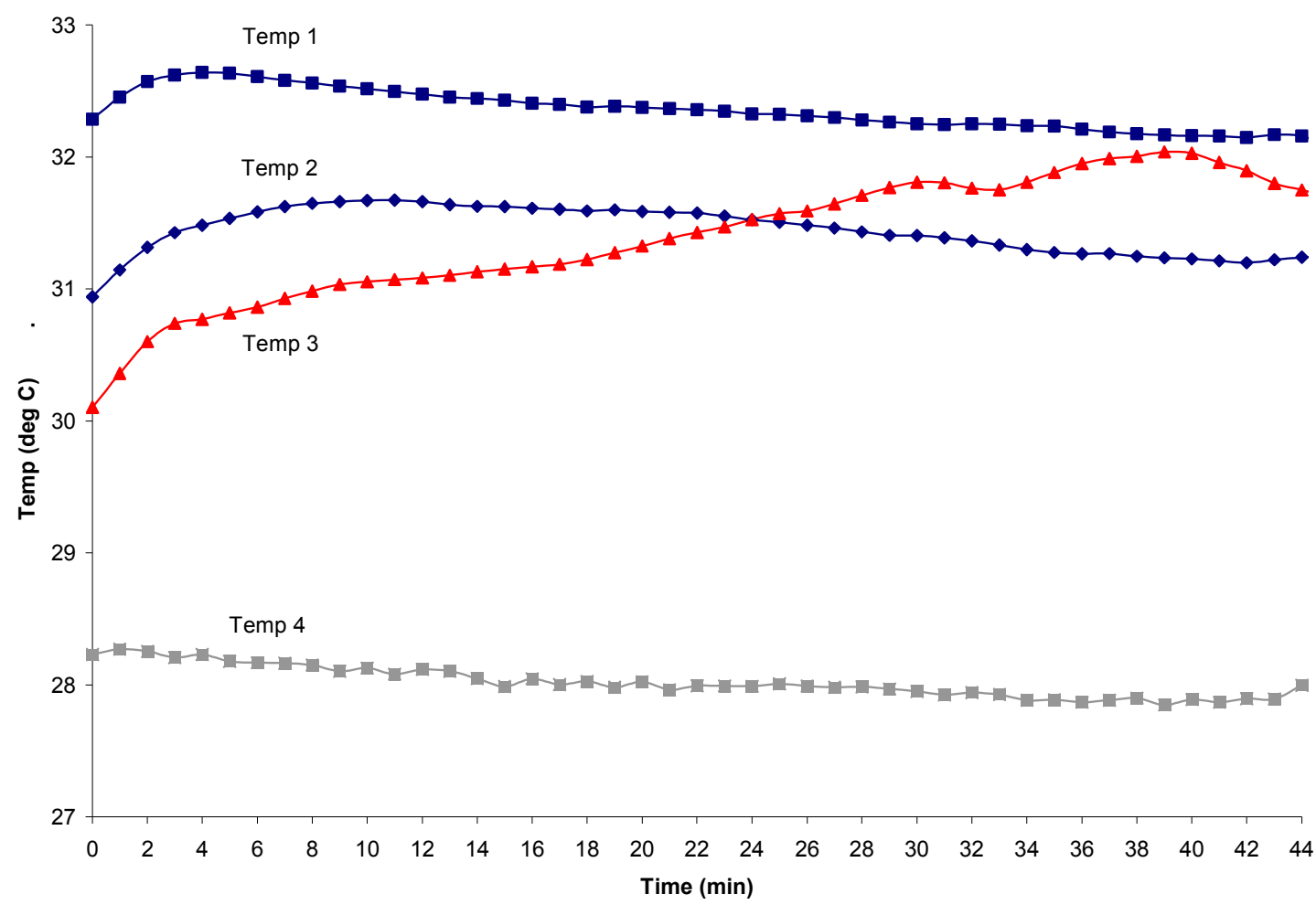

Figure 110: Temperature data for subject 7

When queried after recovering from the adverse event, she told the test director that she had eaten only a Luna bar for lunch, and had oatmeal for breakfast around $10 \mathrm{am}$. (The portion of the test conducted in the pressure chamber was begun approximately at $2 \mathrm{pm}$ ). She additionally informed the test director that she had a fair amount of sleep the night before (7 hours), although the subject was under considerable stress at the time of the experiment. Standing for a long period of time can lead to presyncope in some people (although this subject stated she has never had an event like this occur to her before) and placing her leg in the chamber may have disrupted her circulation, although all data indicates that she experienced no edema other than some minor swelling around her knee. It is possible that some combination of these factors caused the adverse event

\subsection{Subject treatment}

After the test was ended, the subject continued standing inside the pressure chamber, which was at atmospheric pressure, for about 5 minutes. The assistant test director, also an MIT EMT, ensured that she continued speaking and paid close attention to her vital signs. (It should be noted that the team does not believe having medical personnel is necessary for testing; that the assistant test director is an EMT is just a coincidence.) The subject was very nauseous during this time, although she never vomited. She would later note that she had both limited vision and hearing during this 
time period as well. When the subject was able to remove herself from the chamber, she laid down on top of the chamber, at which point the test director and assistant test director worked rapidly to remove the elastic bindings and other material from her leg.

After approximately 3 minutes of the subject lying on top of the chamber, she was able, with significant help of the assistant test director, to sit down in a chair placed near the chamber. The assistant test director told her to place her head between her knees in order to help her feel better.

Within 3-5 minutes after sitting down, the subject was able to stand up without support and respond coherently to questions. The test director talked to the subject as she stood for approximately 25 minutes, while also monitoring her heart rate, which returned to normal. As the subject seemed fully recovered at that point, the test director allowed the subject to leave. It was suggested that she visit MIT Medical either at this time, or if she subsequently felt any further symptoms. She did not feel that this was necessary. The test director contacted the subject after the experiment, and she indicated that she felt fine. She has additionally reported no further symptoms from her participation in the experiment.

The subject was excused from any further involvement in the experiment. 


\section{References}

[1] Annis, J.F., Webb, P. Development of a Space Activity Suit. NASA Contractor Report CR1891 (1971).

[2] Webb, P. The Space Activity Suit: An Elastic Leotard for Extravehicular Activity. Aerospace Medicine 39: 376-383 (1963).

[3] Harris, Gar. L. The Origins and Technology of the advanced Extravehicular Space Suit. American Astronautical Societty History Series, Volume 24, San Diego, California, 2001.

[4] Schmidt, Patricia. An Investigation of Space Suit Mobility with Applications to EVA Operations. Doctoral Thesis, MIT, 2001.

[5] Kozloski, Lillian. U.S. Space Gear: Outfitting the Astronaut. Smithsonian Institution, Washington 1994.

[6] http://www.centennialchallenges.nasa.gov/

[7] Clapp, W. Design and Testing of an advanced spacesuit glove. Master's Thesis, MIT, 1983.

[8] Tourbier, D. et al.. Physiological effects of a mechanical counter-pressure glove. $31^{s t}$ Conference on Environmental Systems, Orlando, FL, 2001.

[9] Tanaka, K. et al. Skin Microvascualr flow during hypobaric exposure with and without a mechanical counterpressure space suit glove. Aviation, Space, and Environmental Medicine, 73(11):1074-1078, 2002.

[10] Tanaka, K. et al. Mechanical counter pressure on the arm counteracts adverse effects of hypobaric pressures. Aviation, Space, and Environemntal Medicine, 74(8): 827, 2003.

[11] Bethke, K. The second-skin approach: Skin strain field analysis and mechanical counterpressure prototyping for advanced spacesuit design. Master's thesis, MIT, 2005.

[12] Wolfrum, N. An Automatic Procedure to Map the Skin Strain Field with Application to Advanced Locomotion Space Suit Desgin. Masters Thesis. TECHNISCHE UNIVERSITÄT MÜNCHEN and MIT (2006).

[13] Pitts, B. Spacesuit: Spacecraft. Master's Thesis, MIT, 2003.

[14] Sim, Z. L. Development of a Mechanical Counter Pressure Bio-Suit System. Masters Thesis. MIT, 2006. 
[15] Iberall, A. The experimental design of a mobile pressure suit. Journal of Basic Engineering, pages 251-264, June 1970.

[16] Carr, Christopher and Loretta Trevino. A First-Order Design Requirement to Prevent Edema in Mechanical Counter-Pressure Space Suit Garments (unpublished).

[17] Nola, G. and Vistnes. L. Differential response of skin and muscle in the experimental procedure of pressure sores. Plastic and Reconstructive Surgery, 66(5): 728-733, 19880.

[18] Schubert, V. and B. Fagrell. Local skin pressure and its effects on skin microcirculation as evaluated by laser-doppler fluxmetry. Clinical Physiology, 9:535-545, 1989.

[19] Patel, S. et al. Temperature effects on surface pressure-induced changes in rat skin perfusion: implications in pressure ulcer development. Journal of Rehabilitation Research and Development, 36(3):189-201, 1999.

[20] Wolthius, R., S. Bergman and A. Nicogrossian. Physioloigcal effects of locally applied reduced pressure in man. Physiological Review, 53 (3):566-595, 1974.

[21] NASA-STD-3000 (Figure 3.3.1.3-1). http://msis.jsc.nasa.gov/ Data from year 2000.

[22] MIT/Draper NASA Concept Exploration and Refinement Study, 2004-05

[23] I-Scan Equilibration and Calibration Practical Suggestions Rev A (company publication). TekScan Inc (South Boston, MA), 10/27/03.

[24] Reddig, M. et al. Physiological limits of underpresure and overpressure in a study of mechanical counter pressure spacesuits. SAE Paper 2003-01-2444, 2003. 